\title{
Decarboxylative Organocatalyzed Addition Reactions of Fluoroacetate Surrogates for the Synthesis of Fluorinated Oxindoles
}

Dominik Zetschok, Lukas Heieck and Helma Wennemers*

ETH Zürich, Laboratory of Organic Chemistry

Department of Chemistry and Applied Biosciences, Vladimir-Prelog-Weg 3, 8093

Zürich Switzerland

E-Mail: Helma.Wennemers@org.chem.ethz.ch

Homepage: http://www.wennemers.ethz.ch 


\section{Table of Content}

1. General Aspects and Materials $\quad$ S2

2. General Procedures S3

3. Optimization of the Reaction Conditions $\quad$ S4

4. Analytic Data of $N$-substituted-Isatins $\quad S 7$

5. Analytical Data of Hydroxyoxindoles $4 \mathbf{a}-\mathbf{v}$ and 5-9 S11

6. Crystal Structure Analysis of Fluorinated Oxindoles $\quad$ S25

7. NMR Spectra $\quad$ S26

8. HPLC/SFC Chromatograms S83

$\begin{array}{ll}\text { 7. References } & \text { S109 }\end{array}$ 


\section{General Aspects and Materials}

All reagents and solvents were purchased in the highest purity available and used without further purification. Reactions requiring inert conditions, were performed under dry nitrogen or argon atmosphere using reactions vessels heated by heat-gun under vacuum. Air- and moisture sensitive compounds and solutions were transferred via syringe or cannula into the reaction vessels through rubber septa. Reactions were stirred magnetically. Yields are determined with spectroscopically pure compounds, unless otherwise stated. Flash column chromatography was performed on silica gel (Merck Kieselgel 60 F254 230-400 mesh). Thin layer chromatography (TLC) was performed on aluminum-backed silica plates $(0.2 \mathrm{~mm}, 60$ F254) or glass-backed silica plates (Merck, Silica Gel 60 F254). TLCs were developed using standard visualizing agents: UV fluorescents (254, $350 \mathrm{~nm})$, iodine, potassium permanganate / $\Delta$, anisaldehyde / $\Delta$, "Magic Seebach" Stain / $\Delta$ (Helv. Chim. Acta 1987, 70, 448). IR: Recorded on neat compounds using FTIR. Only selected peaks are shown. ${ }^{1} \mathrm{H}-\mathrm{NMR}$ : Spectra were recorded on 400,500 or $600 \mathrm{MHz}$ Bruker instruments. Chemical shifts $(\delta \mathrm{H})$ are quoted in parts per million (ppm) and referenced to the appropriate NMR solvent peak(s). ${ }^{13} \mathrm{C}-\mathrm{NMR}$ : Spectra were recorded on a 101, 126 or $150 \mathrm{MHz}$ Bruker instruments. Chemical shifts $(\delta \mathrm{H})$ are quoted in parts per million (ppm) and referenced to the appropriate NMR solvent peak(s). $\mathrm{C}, \mathrm{CH}, \mathrm{CH}_{2}, \mathrm{CH}_{3}, \mathrm{COSY}, \mathrm{HSQC}$ and $\mathrm{HMBC}$ were used where necessary in assigning NMR spectra. ${ }^{19} \mathrm{~F}-\mathrm{NMR}$ : Chemical shifts $(\delta \mathrm{H})$ are quoted in parts per million (ppm) and are not referenced. HRMS: High resolution mass spectra were recorded on an ESI-Q-TOF, MALDITOF-MS or an EI Sector-MS. X-Ray: Full data is listed with the corresponding compounds and cif files. Chiral HPLC: Normal Phase HPLC analyses were performed on an analytical HPLC with a diode array detector SPD-M10A from a Dionex UltiMate 3000 HPLC system (ThermoFisher) using chiral columns (AD-H, AS-H. OD-H, IA, IB-5, IC, ID, IE, IF, IG, IH) (250 mm x 4.6 $\mathrm{mm}$ ) from Daicel under the reported conditions. Chiral SFC: SFC analyses were performed using an analytical SFC with a diode array detector ACQUITY-UPLC-PDA from Waters using chiral columns (Trefoil, AS, AD, IC, Whelk, OD, OJ, IA) (150 mm x $30 \mathrm{~mm}$ ) from Daicel or Waters under the reported conditions. Racemic samples were obtained using 1-(3,5bis(trifluoromethyl)phenyl)-3-(2-(dimethylamino)ethyl)thiourea $(20 \mathrm{~mol} \%)$ in acetone $(0.1 \mathrm{M})$. Diasteromeric rations were determined by ${ }^{1} \mathrm{H}$-NMR from the ratios of the $\mathrm{CFH}$ peaks. Catalysts were synthesized according to previously published procedures ${ }^{1}$ or purchased if commercially available. F-MAHTs were synthesized following previously published procedures. $^{2}$ 


\section{General Procedures}

\section{General Procedure 1: Preparation of methyl protected isatins}

Isatin $(6.0 \mathrm{mmol})$ was dissolved in $\mathrm{DMF}(0.75 \mathrm{M})$ and $\mathrm{K}_{2} \mathrm{CO}_{3}$ (2.5 equiv.) was added. The solution was stirred for 5 minutes at room temperature and subsequently methyl iodide (3.0 equiv.) was added dropwise. The mixture was stirred overnight at room temperature. After TLC analysis indicated full conversion, the reaction was quenched by addition of water $(20 \mathrm{~mL})$. The phases were separated, the aqueous phase was extracted three times with $\mathrm{CH}_{2} \mathrm{Cl}_{2}(30$ $\mathrm{mL}$ ). The combined organic phases were washed with brine, dried over $\mathrm{Na}_{2} \mathrm{SO}_{4}$ and concentrated under reduced pressure. The crude product was purified by flash column chromatography (ethyl acetate / $n$-hexane) or recrystallized from $\mathrm{CH}_{2} \mathrm{Cl}_{2}$ / $n$-hexane.

\section{General Procedure 2: Stereoselective addition reaction}

Isatin was added to a vial. The vial was closed and evacuated three times and backfilled with dry nitrogen. Then, catalyst (20 mol\%) was added as a stock solution in acetone (or the respective solvent used for the reaction) and the mixture was cooled to $0^{\circ} \mathrm{C}$. Subsequently, FMAHT (1.5 equiv.) was added as stock solution and the solution (25 mM with respect to the isatin) was stirred at $10^{\circ} \mathrm{C}$ for $24-64 \mathrm{~h}$. After the reaction reached full conversion, the solution was filtered through a plug of silica and evaporated onto celite. The crude product was purified by flash column chromatography (ethyl acetate / $n$-hexane). 


\section{Optimization of the Reaction Conditions}

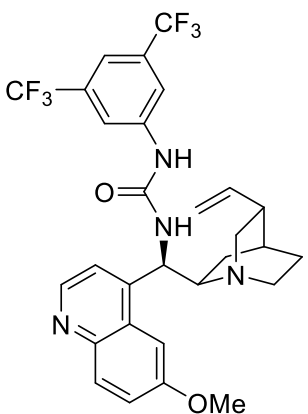

A eQDU

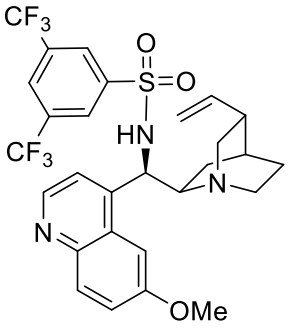

D eQDSA

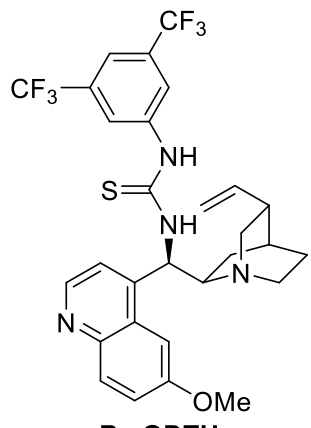

B eQDTU

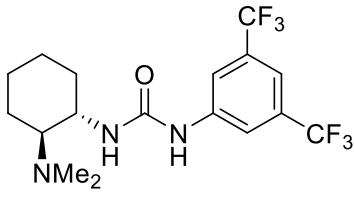

E Takemoto catalyst

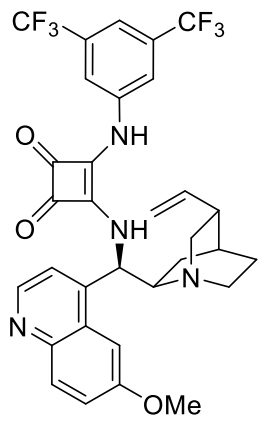

C eQDSqA

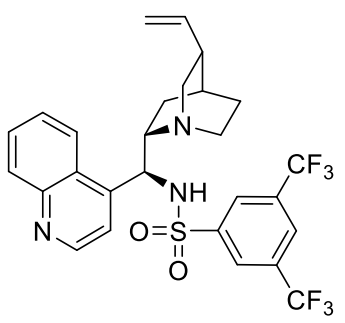

F eCDSA

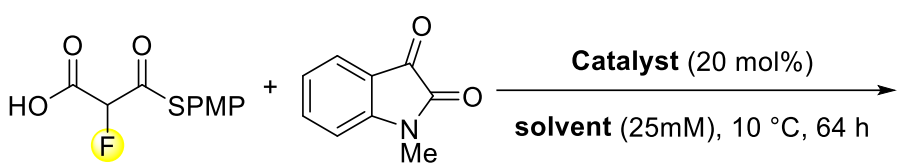

1

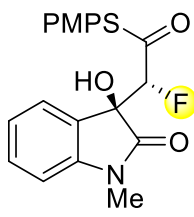

4a

Table S1: Catalyst and solvent optimization

$\begin{array}{cccccc}\text { Entry } & \text { Catalyst } & \text { Solvent } & \text { Conversion } & \mathrm{dr} & e e \\ 1 & \mathrm{~A} & \mathrm{THF} & 91 \% & 1.1: 1 & -61 \% \\ 2 & \mathrm{~B} & \mathrm{THF} & 100 \% & 1.3: 1 & -30 \% \\ 3 & \mathrm{C} & \mathrm{THF} & 100 \% & 1.2: 1 & 14 \% \\ 4 & \mathrm{D} & \mathrm{THF} & 100 \% & 1.5: 1 & -82 \% \\ 5 & \mathrm{E} & \mathrm{THF} & 100 \% & 2.6: 1 & -52 \% \\ 6 & \mathrm{D} & \mathrm{CH}_{2} \mathrm{Cl}_{2} & 12 \% & 1.1: 1 & -82 \% \\ 7 & \mathrm{D} & \text { toluene } & 8 \% & 1.4: 1 & -82 \% \\ 8 & \mathrm{D} & \mathrm{Et}_{2} \mathrm{O} & 62 \% & 1.2: 1 & -82 \% \\ 9 & \mathrm{D} & \mathrm{MeCN}_{1} & 68 \% & 1.6: 1 & -66 \% \\ 10 & \mathrm{D} & \mathrm{MeOH}_{1} & 6 \% & 2.4: 1 & -16 \% \\ 11 & \mathrm{D} & \text { EtOAc } & 47 \% & 1.3: 1 & -78 \% \\ 12 & \mathrm{D} & \text { acetone } & 100 \% & 1.4: 1 & -90 \% \\ 13 & \mathrm{D} & \text { Cyclohexanone } & 88 \% & 1: 1.5 & -88 \% \\ 14 & \mathrm{D} & \text { Acetophenone } & 67 \% & 1: 1 & -73 \% \\ 15 & \mathrm{D} & \text { 3-Methylbutanon } & 97 \% & 1: 1 & -88 \% \\ 16 & \mathrm{D} & \text { Isophorone } & 77 \% & 1: 1 & -82 \%\end{array}$



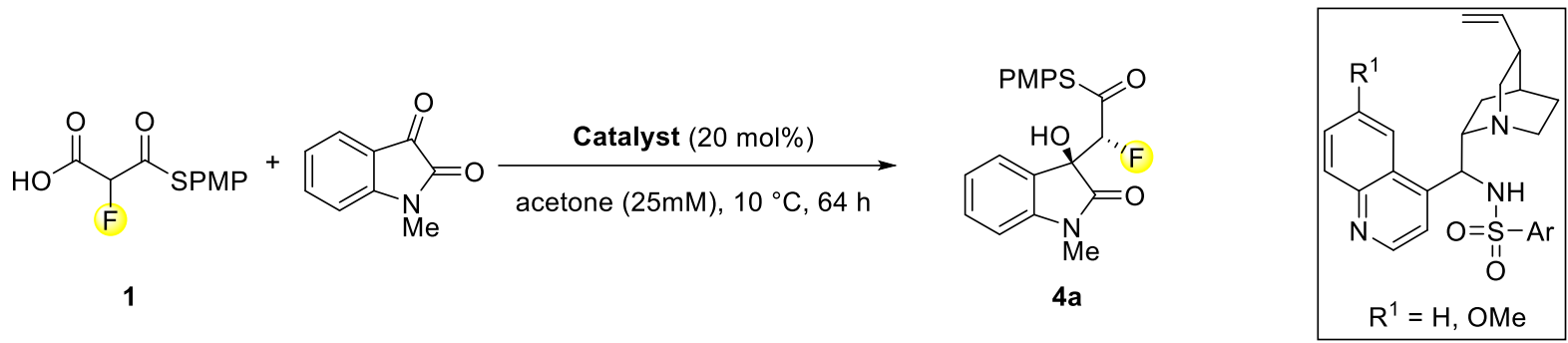

Table S2: Sulfonamide catalyst optimization

$\begin{array}{cccccccc}\text { Entry } & \text { Catalyst } & \mathrm{R}^{1} & \mathrm{Ar} & \text { Additive } & \text { Conversion } & \mathrm{dr} & \text { ee } \\ 1 & \text { eQDSA } & \mathrm{OMe} & 3,5-\left(\mathrm{CF}_{3}\right)_{2}-\mathrm{Ph} & - & 100 \% & 1.4: 1 & -90 \% \\ 2 & \text { eQNSA } & \mathrm{OMe} & 3,5-\left(\mathrm{CF}_{3}\right)_{2}-\mathrm{Ph} & - & 90 \% & 1.3: 1 & 84 \% \\ 3 & \text { eCNSA } & \mathrm{H} & 3,5-\left(\mathrm{CF}_{3}\right)_{2}-\mathrm{Ph} & - & 96 \% & 1.2: 1 & -85 \% \\ \mathbf{4} & \mathbf{F} \text { - eCDSA } & \mathrm{H} & \mathbf{3 , 5}-\left(\mathrm{CF}_{3}\right)_{2}-\mathrm{Ph} & - & \mathbf{1 0 0} \% & \mathbf{1 . 5}: 1 & \mathbf{9 2} \% \\ 5 & \text { eCDSA } & \mathrm{H} & 4-\mathrm{Me}-\mathrm{Ph} & - & 96 \% & 1.6: 1 & 73 \% \\ 6 & \text { eCDSA } & \mathrm{H} & 4-\mathrm{OMe}-\mathrm{Ph} & - & 98 \% & 1.6: 1 & 71 \% \\ 7 & \text { eCDSA } & \mathrm{H} & 4-\mathrm{CF}_{3}-\mathrm{Ph} & - & 100 \% & 1.4: 1 & 86 \% \\ 8 & \text { eCDSA } & \mathrm{H} & 2,4,6-(\mathrm{Pr})_{3}-\mathrm{Ph} & - & 100 \% & 1.5: 1 & 79 \% \\ 9 & \text { eCDSA } & \mathrm{H} & \text { 1-Naph } & - & 95 \% & 1.2: 1 & 79 \% \\ 10 & \text { eCDSA } & \mathrm{H} & \text { 2-Naph } & - & 98 \% & 1.7: 1 & 78 \% \\ 11 & \text { eCDSA } & \mathrm{H} & \text { 8-Quinoline } & - & 95 \% & 3.6: 1 & 8 \%\end{array}$




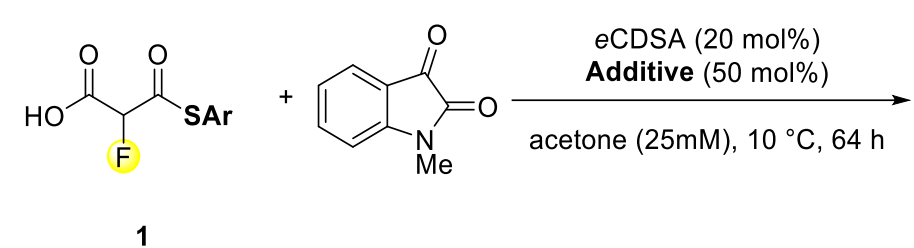

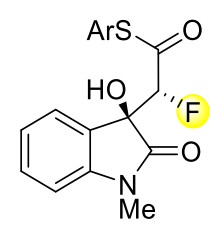

$4 a$

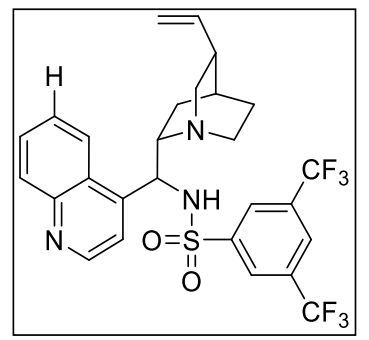

Table S3: F-MAHT Optimization

\begin{tabular}{|c|c|c|c|c|c|c|}
\hline Entry & Catalyst & $\mathrm{Ar}$ & Additive & Conversion & $d r$ & ee \\
\hline 1 & $\mathbf{F}$ & 4-OMe-Ph & - & $100 \%$ & $1.5: 1$ & $92 \%$ \\
\hline 2 & $\mathrm{~F}$ & 3-OMe-Ph & - & $69 \%$ & 1.8:1 & $84 \%$ \\
\hline 3 & $\mathrm{~F}$ & 1-OMe-Ph & - & $42 \%$ & $1.3: 1$ & $-85 \%$ \\
\hline 4 & $F$ & 4-F-Ph & - & $45 \%$ & $1.5: 1$ & $92 \%$ \\
\hline 5 & $\mathrm{~F}$ & 2-F-Ph & - & $100 \%$ & $3.4: 1$ & $73 \%$ \\
\hline 6 & $F$ & $2,6-\mathrm{Cl}_{2}-\mathrm{Ph}$ & - & $67 \%$ & $2.0: 1$ & $71 \%$ \\
\hline 7 & $\mathrm{~F}$ & $\mathrm{Ph}$ & - & $87 \%$ & $1.6: 1$ & $86 \%$ \\
\hline 8 & $\mathrm{~F}$ & $\mathrm{Bn}$ & - & $51 \%$ & $1.3: 1$ & $79 \%$ \\
\hline 9 & $\mathrm{~F}$ & Cy & - & $29 \%$ & 1.1:1 & $79 \%$ \\
\hline 10 & $\mathrm{~F}$ & 4-OMe-Ph & Imidazole & $85 \%$ & $1.6: 1$ & $28 \%$ \\
\hline 11 & $\mathrm{~F}$ & 4-OMe-Ph & NMM & $79 \%$ & $1.8: 1$ & $35 \%$ \\
\hline 12 & $\mathrm{~F}$ & 4-OMe-Ph & pyridine & $84 \%$ & $1.6: 1$ & $62 \%$ \\
\hline 13 & $\mathrm{~F}$ & 4-OMe-Ph & DMAP & $83 \%$ & $1.8: 1$ & $31 \%$ \\
\hline 14 & $F$ & 4-OMe-Ph & Triethylamine & $72 \%$ & $2.2: 1$ & $59 \%$ \\
\hline 15 & $\mathrm{~F}$ & 4-OMe-Ph & Acetic acid & 56 & $1.7: 1$ & $89 \%$ \\
\hline 16 & $\mathrm{~F}$ & 4-OMe-Ph & $\mathrm{PhCOOH}$ & 75 & $1.5: 1$ & $89 \%$ \\
\hline 17 & $F$ & 4-OMe-Ph & DMSO & 69 & $1.5: 1$ & $92 \%$ \\
\hline 18 & $\mathrm{~F}$ & 4-OMe-Ph & molsieves & 100 & $1.3: 1$ & $57 \%$ \\
\hline
\end{tabular}




\section{Analytical Data of $\mathbf{N}$-substituted-Isatins}

\section{1,5-dimethylindoline-2,3-dione}

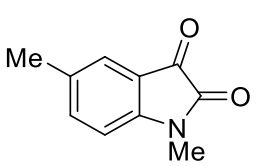

Prepared according to general procedure 1, purified by flash column chromatography (ethyl acetate / $n$-hexane) and obtained as orange solid (2.32 g, $86 \%)$.

$\boldsymbol{R}_{\mathbf{f}} 0.33$ (EtOAc / $n$-hexane, 3:7); ${ }^{1} \mathbf{H}$ NMR $\left(400 \mathrm{MHz}, \mathrm{CDCl}_{3}, 25^{\circ} \mathrm{C}\right) \delta=7.44-7.33(\mathrm{~m}, 2 \mathrm{H}$, $\mathrm{ArH}), 6.78(\mathrm{~d}, \mathrm{~J}=8.7,1 \mathrm{H}, \mathrm{ArH}), 3.23\left(\mathrm{~s}, 3 \mathrm{H}, \mathrm{NCH}_{3}\right), 2.34\left(\mathrm{~s}, 3 \mathrm{H}, \mathrm{ArCH}_{3}\right)$.

Data in accordance to literature. ${ }^{4}$

\section{5-methoxy-1-methylindoline-2,3-dione}

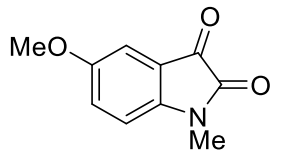

Prepared according to general procedure 1, recrystallized from $\mathrm{CH}_{2} \mathrm{Cl}_{2}$ and obtained as a violet solid (2.05 g, $76.0 \%)$.

$\boldsymbol{R}_{\mathrm{f}} 0.5$ (EtOAc / $n$-hexane, 1:1); ${ }^{\mathbf{H}} \mathbf{N M R}\left(400 \mathrm{MHz}, \mathrm{CDCl}_{3}, 25^{\circ} \mathrm{C}\right) \delta=7.20-$ $7.07(\mathrm{~m}, 2 \mathrm{H}, \mathrm{ArH}), 6.81(\mathrm{~d}, \mathrm{~J}=9.5,1 \mathrm{H}, \mathrm{ArH}), 3.81\left(\mathrm{~s}, 3 \mathrm{H}, \mathrm{OCH}_{3}\right), 3.22\left(\mathrm{~s}, 3 \mathrm{H}, \mathrm{NCH}_{3}\right)$.

Data in accordance to literature. ${ }^{5}$

\section{5-fluoro-1-methylindoline-2, 3-dione}

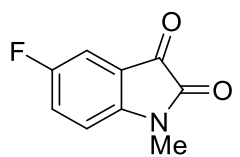

Prepared according to general procedure 1, purified by flash column chromatography (ethyl acetate / $n$-hexane) and obtained as a red solid (747 mg, $28 \%$ ).

$\boldsymbol{R}_{\mathrm{f}} 0.39$ (EtOAc / $n$-hexane, 1:1); ${ }^{1} \mathbf{H}$ NMR $\left(400 \mathrm{MHz}, \mathrm{CDCl}_{3}, 25^{\circ} \mathrm{C}\right) \delta=7.39-7.28(\mathrm{~m}, 2 \mathrm{H}$, $\mathrm{ArH}), 6.91-6.81(\mathrm{~m}, 1 \mathrm{H}, \mathrm{ArH}), 3.26\left(\mathrm{~s}, 3 \mathrm{H}, \mathrm{NCH}_{3}\right) .{ }^{19} \mathrm{~F}$ NMR $\left(377 \mathrm{MHz}, \mathrm{CDCl}_{3}, 25^{\circ} \mathrm{C}\right) \delta=-$ $118.06--118.16(\mathrm{~m})$.

Data in accordance to literature. ${ }^{4}$

\section{5-chloro-1-methylindoline-2, 3-dione}

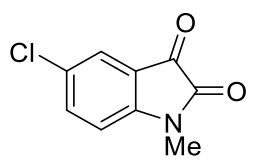

Prepared according to general procedure 1, purified by flash column chromatography (ethyl acetate / $n$-hexane) and obtained as an orange solid (831 mg, $77 \%$ ).

$\boldsymbol{R}_{\mathrm{f}} 0.28$ (EtOAc / $n$-hexane, 3:7); ${ }^{1} \mathbf{H}$ NMR $\left(400 \mathrm{MHz}, \mathrm{CDCl}_{3}, 25^{\circ} \mathrm{C}\right) \delta=7.62-7.55(\mathrm{~m}, 2 \mathrm{H}$, ArH), $6.86(\mathrm{~d}, \mathrm{~J}=9.0,1 \mathrm{H}, \mathrm{ArH}), 3.26\left(\mathrm{~s}, 3 \mathrm{H}, \mathrm{NCH}_{3}\right)$.

Data in accordance to literature. ${ }^{4}$

\section{5-bromo-1-methylindoline-2,3-dione}

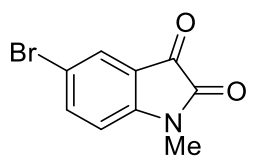

Prepared according to general procedure 1, purified by flash column chromatography (ethyl acetate / $n$-hexane) and obtained as an orange solid $(1.77 \mathrm{~g}, 67 \%)$.

$\mathbf{R}_{\mathrm{f}} 0.43$ (EtOAc / $n$-hexane, $\left.1: 1\right) ;{ }^{1} \mathrm{H}$ NMR $\left(400 \mathrm{MHz}, \mathrm{CDCl}_{3}, 25^{\circ} \mathrm{C}\right) \delta=7.73(\mathrm{~d}, \mathrm{~J}=2.1,1 \mathrm{H}, \mathrm{ArH})$, $7.71(\mathrm{~d}, \mathrm{~J}=1.3,1 \mathrm{H}, \mathrm{ArH}), 6.81$ (dd, $1 \mathrm{H}, \mathrm{ArH}), 3.25\left(\mathrm{~s}, 3 \mathrm{H}, \mathrm{NCH}_{3}\right)$.

Data in accordance to literature. ${ }^{5}$ 


\section{4-fluoro-1-methylindolin-2,3-dione}

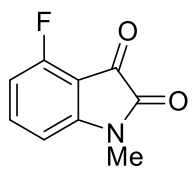

Prepared according to general procedure 1, recrystallized from $\mathrm{CH}_{2} \mathrm{Cl}_{2}$ and obtained as a dark orange solid (957 mg, 89\%).

$\mathbf{R}_{\mathbf{f}} 0.20$ (EtOAc / $n$-hexane, 1:1). M.P. $175-177^{\circ} \mathrm{C}$. IR $\mathbf{v}_{\max }$ (neat) $/ \mathrm{cm}^{-1} 3066,2920$, 2359, 2339, 1725, 1618, 1490, 1468, 1352, 1301, 1248, 1205, 1155, 1055, 904, 788; ${ }^{1} \mathrm{H}$ NMR $\left(500 \mathrm{MHz}, \mathrm{CDCl}_{3}, 25^{\circ} \mathrm{C}\right) \delta 7.60$ (ddd, $\left.J=8.6,7.8,5.5 \mathrm{~Hz}, 1 \mathrm{H}, \mathrm{ArH}\right), 6.79$ (td, $J=8.5,0.6 \mathrm{~Hz}$, $1 \mathrm{H}, \mathrm{ArH}), 6.70$ (dd, $J=7.9,0.6 \mathrm{~Hz}, 1 \mathrm{H}, \mathrm{ArH}), 3.27$ (s, 3H, NCH$)_{3}{ }^{13} \mathrm{C} \mathrm{NMR}\left(126 \mathrm{MHz} \mathrm{CDCl}_{3}\right.$, $\left.25^{\circ} \mathrm{C}\right) \delta 179.0,159.9,157.8\left(\mathrm{~d},{ }^{2} J \mathrm{C}-\mathrm{F}=6.5 \mathrm{~Hz}\right), 152.0\left(\mathrm{~d},{ }^{2} J \mathrm{C}-\mathrm{F}=5.8 \mathrm{~Hz}\right), 140.8,112.1$ (d, $\left.{ }^{1} \mathrm{~J} \mathrm{C}-\mathrm{F}=19.8 \mathrm{~Hz}\right), 106.0\left(\mathrm{~d},{ }^{3} \mathrm{~J} \mathrm{C}-\mathrm{F}=3.3 \mathrm{~Hz}\right), 105.7\left(\mathrm{~d},{ }^{2} \mathrm{~J} \mathrm{C}-\mathrm{F}=18.3 \mathrm{~Hz}\right), 26.9 .{ }^{19} \mathrm{~F}$ NMR (471 $\mathrm{MHz}, \mathrm{CDCl}_{3}, 25^{\circ} \mathrm{C}$ ) $\delta-106.14$ (dd, $J=8.2,5.5 \mathrm{~Hz}$ ). HRMS (ESI+): m/z [M+H] ${ }^{+}$Calcd. for $\mathrm{C}_{9} \mathrm{H}_{7} \mathrm{FNO}_{2}$ : 180.0455; Found: 180.0457 .

\section{4-chloro-1-methylindolin-2,3-dione}<smiles>O=C1C(=O)N([N+](=O)[O-])c2cccc(Cl)c21</smiles>

Prepared according to general procedure 1, recrystallized from $\mathrm{CH}_{2} \mathrm{Cl}_{2}$ and obtained as an red solid $(1.07 \mathrm{~g}, 91 \%)$.

$\mathbf{R}_{\mathbf{f}} 0.21$ (EtOAc / $n$-hexane, 1:1). M.P. $192-194^{\circ} \mathrm{C}$. IR $\mathbf{v}_{\max }$ (neat) $/ \mathrm{cm}^{-1} 3077,3023$, 2921, 2360, 2339, 2106, 2082, 1727, 1582, 1446, 1347, 1286, 1206, 1163, 1119, 1041, 863, 780. ${ }^{1} \mathrm{H}$ NMR $\left(500 \mathrm{MHz}, \mathrm{CDCl}_{3}, 2^{\circ}{ }^{\circ} \mathrm{C}\right) \delta 7.54-7.47(\mathrm{~m}, 1 \mathrm{H}, \mathrm{ArH}), 7.07$ (dd, $J=8.2,0.7 \mathrm{~Hz}$, $1 \mathrm{H}, \mathrm{ArH}), 6.80$ (dd, $J=7.9,0.7 \mathrm{~Hz}, 1 \mathrm{H}, \mathrm{ArH}), 3.26\left(\mathrm{~s}, 3 \mathrm{H}, \mathrm{NCH}_{3}\right) \cdot{ }^{13} \mathrm{C} \mathrm{NMR}\left(126 \mathrm{MHz}, \mathrm{CDCl}_{3}\right.$, $\left.25^{\circ} \mathrm{C}\right) \delta 180.3,157.5,152.7,138.7,134.0,125.6,114.7,108.3,26.6$. HRMS (ESI+): $\mathrm{m} / \mathrm{z}$ $[\mathrm{M}+\mathrm{H}]^{+}$Calcd. for $\mathrm{C}_{9} \mathrm{H}_{7} \mathrm{CINO}_{2}$ : 196.0160; Found: 196.0159.

\section{4-bromo-1-methylindolin-2,3-dione}

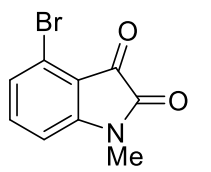

Prepared according to general procedure 1, recrystallized from $\mathrm{CH}_{2} \mathrm{Cl}_{2}$ and obtained as an orange solid (1.70 g, $69 \%)$.

$\mathbf{R}_{\mathrm{f}} 0.22$ (EtOAc / $n$-hexane, 1:1). M.P. 202-204 ${ }^{\circ} \mathrm{C}$. IR $\mathrm{v}_{\max }$ (neat) $/ \mathrm{cm}^{-1} 3442,3075$, 3021, 2939, 1736, 1727, 1593, 1578, 1449, 1346, 1302, 1285, 1207, 1163, 1114, 1037, 864, 779. ${ }^{1} \mathrm{H}$ NMR $\left(500 \mathrm{MHz}, \mathrm{CDCl}_{3}, 2^{\circ}{ }^{\circ} \mathrm{C}\right) \delta 7.46-7.39(\mathrm{~m}, 1 \mathrm{H}, \mathrm{ArH}), 7.25$ (dd, $J=8.2,0.7 \mathrm{~Hz}$, $1 \mathrm{H}, \mathrm{ArH}), 6.84(\mathrm{dd}, J=7.9,0.7 \mathrm{~Hz}, 1 \mathrm{H}, \mathrm{ArH}), 3.25\left(\mathrm{~s}, 3 \mathrm{H}, \mathrm{NCH}_{3}\right) .{ }^{13} \mathrm{C} \mathrm{NMR}\left(126 \mathrm{MHz}, \mathrm{CDCl}_{3}\right.$, $\left.25^{\circ} \mathrm{C}\right) \delta 180.8,157.4,153.1,138.5,128.7,121.7,116.4,108.8,26.5$. HRMS (ESI+): $\mathrm{m} / \mathrm{z}$ $[\mathrm{M}+\mathrm{H}]^{+}$Calcd. for $\mathrm{C}_{9} \mathrm{H}_{7} \mathrm{BrNO}_{2}$ : 239.9655; Found: 239.9655.

\section{4-phenyl-1-methylindolin-2,3-dione}

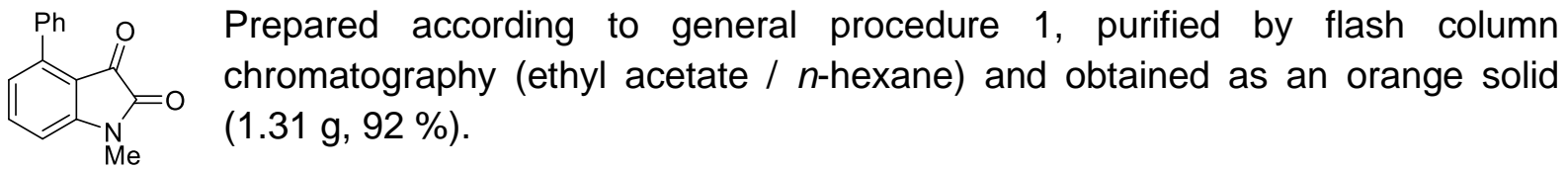

$\mathbf{R}_{\mathbf{f}} 0.28$ (EtOAc / $n$-hexane, 1:1). M.P. 176-178 ${ }^{\circ} \mathrm{C}$. IR $\mathbf{V}_{\max }$ (neat) $/ \mathrm{cm}^{-1} 3459,3074,3056,3037$, 2940, 2360, 1738, 1721, 1603, 1587, 1569, 1502, 1479, 1460, 1435, 1358, 1310, 1202, 1162 , 1088. ${ }^{1} \mathrm{H}$ NMR $\left(500 \mathrm{MHz}, \mathrm{CDCl}_{3}, 2^{\circ} 5^{\circ} \mathrm{C}\right) \delta 7.60$ (t, $\left.J=7.9 \mathrm{~Hz}, 1 \mathrm{H}, \mathrm{ArH}\right), 7.57-7.49(\mathrm{~m}, 2 \mathrm{H}$, ArH), $7.52-7.39(\mathrm{~m}, 3 \mathrm{H}, \mathrm{ArH}), 7.10(\mathrm{dd}, J=7.9,0.8 \mathrm{~Hz}, 1 \mathrm{H}, \mathrm{ArH}), 6.86(\mathrm{dd}, J=7.9,0.9 \mathrm{~Hz}$, $1 \mathrm{H}, \mathrm{ArH}), 3.29\left(\mathrm{~s}, 3 \mathrm{H}, \mathrm{NCH}_{3}\right) .{ }^{13} \mathrm{C}$ NMR $\left(126 \mathrm{MHz} \mathrm{CDCl}_{3}, 25^{\circ} \mathrm{C}\right) \delta 182.1,158.1,152.4,143.3$, 137.8, 136.1, 135.2, 129.3, 129.1, 128.4, 128.2, 126.0, 113.9, 108.6, 26.5. HRMS (ESI+): m/z $[\mathrm{M}+\mathrm{H}]^{+}$Calcd. $\mathrm{C}_{15} \mathrm{H}_{12} \mathrm{NO}_{2}$ : 238.0863; Found: 238.0863. 


\section{4,7-dichloro-1-methylindoline-2,3-dione}<smiles>O=C1C(=O)N([O-])c2c(Cl)ccc(Cl)c21</smiles>

Prepared according to general procedure 1, recrystallized from $\mathrm{CH}_{2} \mathrm{Cl}_{2}$ and obtained as an orange solid (542 mg, $51 \%$ ).

$\mathbf{R}_{\mathbf{f}} 0.54$ (EtOAc / $n$-hexane, 1:1). M.P. $170-171^{\circ} \mathrm{C}$. IR $v_{\max }$ (neat) $/ \mathrm{cm}^{-1} 3453,3066$, 3016, 2956, 2360, 1733, 1581, 1470, 1440, 1291, 1202, 1175, 1130, 1046. ${ }^{1} \mathbf{H}$ NMR $\left(500 \mathrm{MHz}, \mathrm{CDCl}_{3}, 25^{\circ} \mathrm{C}\right) \delta 7.43(\mathrm{~d}, J=8.7 \mathrm{~Hz}, 1 \mathrm{H}, \mathrm{ArH}), 7.01(\mathrm{~d}, J=8.7 \mathrm{~Hz}, 1 \mathrm{H}, \operatorname{ArH})$, $3.64\left(\mathrm{~s}, 3 \mathrm{H}, \mathrm{NCH}_{3}\right) .{ }^{13} \mathrm{C}$ NMR $\left(126 \mathrm{MHz}, \mathrm{CDCl}_{3}, 25^{\circ} \mathrm{C}\right) \delta 179.5,157.8,147.8,140.5,132.9$, 126.5, 116.8, 115.9, 30.0. HRMS (ESI+): $\mathrm{m} / \mathrm{z}[\mathrm{M}+\mathrm{H}]^{+}$Calcd. for $\mathrm{C}_{9} \mathrm{H}_{6} \mathrm{Cl}_{2} \mathrm{NO}_{2}: 229.9770\left[\mathrm{M}_{+} \mathrm{H}^{+}\right]$; Found: 229.9769.

\section{1-benzyl-4,7-dichloroindoline-2,3-dione}<smiles>O=C1[NH2+]c2c(Cl)ccc(Cl)c2C1=O</smiles>

Prepared according to general procedure 1, recrystallized from $\mathrm{CH}_{2} \mathrm{Cl}_{2}$ and obtained as an orange solid (1.83 g, $43 \%)$.

$\mathbf{R}_{\mathbf{f}} 0.59$ (EtOAc / $n$-hexane, 1:1). ${ }^{1} \mathbf{H}$ NMR $\left(400 \mathrm{MHz}, \mathrm{CDCl}_{3}, 25^{\circ} \mathrm{C}\right) \delta=7.38(\mathrm{~d}$, $J=8.7,1 \mathrm{H}, \operatorname{ArH}), 7.35-7.24(\mathrm{~m}, 5 \mathrm{H}, \operatorname{ArH}), 7.02(\mathrm{~d}, \mathrm{~J}=8.7,1 \mathrm{H}, \mathrm{ArH}), 5.41-5.38$ $\left(\mathrm{m}, 2 \mathrm{H}, \mathrm{NCH}_{2} \mathrm{Ar}\right)$.

Data in accordance to literature. ${ }^{6}$

\section{1-methyl-5-(trifluoromethoxy)indoline-2,3-dione}

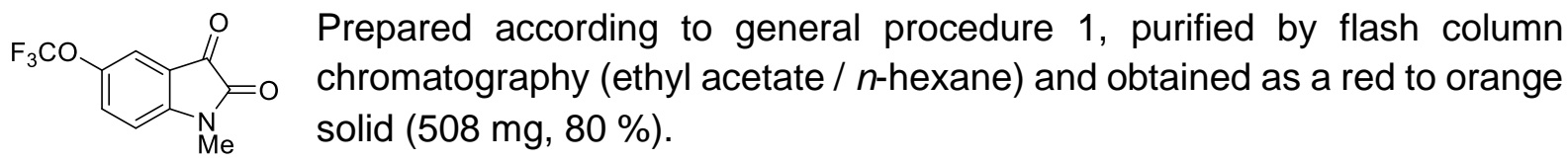

$\mathbf{R}_{\mathbf{f}} 0.34$ (EtOAc / n-hexane, 1:1). M.P. 112-114 ${ }^{\circ} \mathrm{C} \mathrm{IR} \mathrm{v}_{\max }$ (neat)/ $\mathrm{cm}^{-1} 3465,3096,3065,3043$, 2949, 2359, 1737, 1614, 1486, 1471, 1264, 1207, 1156, 1107. ${ }^{1} \mathbf{H}$ NMR $\left(500 \mathrm{MHz}^{-\mathrm{CDCl}_{3}}\right.$, $\left.25^{\circ} \mathrm{C}\right) \delta 7.47(\mathrm{~m}, 2 \mathrm{H}, \mathrm{ArH}), 6.94(\mathrm{dd}, J=7.9,1.1 \mathrm{~Hz}, 1 \mathrm{H}, \mathrm{ArH}), 3.27\left(\mathrm{~s}, 3 \mathrm{H}, \mathrm{NCH}_{3}\right) .{ }^{13} \mathrm{C} \mathrm{NMR}$ $\left(126 \mathrm{MHz}, \mathrm{CDCl}_{3}, 25^{\circ} \mathrm{C}\right) 182.5,158.0,149.9,145.5$ (q, $\left.{ }^{3} \mathrm{~J} \mathrm{C}-\mathrm{F}=2.2 \mathrm{~Hz}\right), 131.3,120.5\left(\mathrm{q},{ }^{1} \mathrm{~J}\right.$ $\left.\mathrm{CF}_{3}=258.3 \mathrm{~Hz}\right), 118.5\left(\mathrm{~d},{ }^{4} \mathrm{~J} \mathrm{C}-\mathrm{F}=1.0 \mathrm{~Hz}\right), 118.0,111.2,26.5 . .{ }^{19} \mathrm{~F}$ NMR $\left(471 \mathrm{MHz}, \mathrm{CDCl}_{3}\right.$, $\left.25^{\circ} \mathrm{C}\right) \delta$-58.61. HRMS (ESI+): $\mathrm{m} / \mathrm{z}[\mathrm{M}+\mathrm{H}]^{+}$Calcd. for $\mathrm{C}_{10} \mathrm{H}_{7} \mathrm{~F}_{3} \mathrm{NO}_{3}: 246.0373$; Found: 246.0374.

\section{6-chloro-1-methylindoline-2, 3-dione}

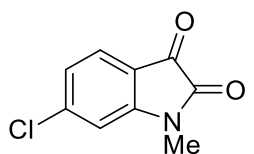

Prepared according to general procedure 1, purified by flash column chromatography (ethyl acetate / $n$-hexane) and obtained as an orange solid $(2.14 \mathrm{~g}, 79 \%)$.

$\boldsymbol{R}_{\mathrm{f}} 0.59$ (EtOAc / $n$-hexane, 3:7). ${ }^{1} \mathbf{H}$ NMR $\left(400 \mathrm{MHz}, \mathrm{CDCl}_{3}, 25^{\circ} \mathrm{C}\right) \delta=7.55(\mathrm{~d}, \mathrm{~J}=8.0,1 \mathrm{H}, \mathrm{ArH})$, 7.11 (dd, J=8.0, 1.7, $1 \mathrm{H}, \mathrm{ArH}), 6.91$ (d, J=1.7, $1 \mathrm{H}, \mathrm{ArH}), 3.25$ (s, 3H, NCH$)_{3}$.

Data in accordance to literature. ${ }^{5}$ 


\section{1-methyl-7-(trifluoromethyl)indoline-2,3-dione}

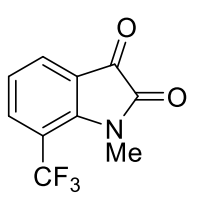

Prepared according to general procedure 1, recrystallized from $\mathrm{CH}_{2} \mathrm{Cl}_{2}$ and obtained as an orange solid ( $365 \mathrm{mg}, 57 \%$ ).

$\mathbf{R}_{\mathbf{f}} 0.86$ (EtOAc / $n$-hexane, 3:7). M.P. $184-187^{\circ} \mathrm{C} . \mathbf{v}_{\max }$ (neat) $/ \mathbf{c m}^{-1} 3101,2967$, 1738, 1590, 1446, 1100. ${ }^{1} \mathrm{H}$ NMR $\left(400 \mathrm{MHz} \mathrm{CDCl}_{3}, 25^{\circ} \mathrm{C}\right) \delta=7.89$ (dq, J=8.1, 1.4, 0.6, $1 \mathrm{H}, \mathrm{ArH}), 7.85-7.78(\mathrm{~m}, 1 \mathrm{H}, \mathrm{ArH}), 7.25-7.22(\mathrm{~m}, 1 \mathrm{H}, \operatorname{ArH}), 3.47(\mathrm{q}, \mathrm{J}=2.4,3 \mathrm{H}$, $\left.\mathrm{NCH}_{3}\right) .{ }^{13} \mathrm{C}$ NMR $\left(101 \mathrm{MHz}, \mathrm{CDCl}_{3}, 25^{\circ} \mathrm{C}\right) \delta=181.8,158.8,149.3,135.7$ (q, $\left.{ }^{2} \mathrm{~J} \mathrm{C}-\mathrm{F}=5.9 \mathrm{~Hz}\right)$, 128.7, 123.5, $122.8\left(\mathrm{q},{ }^{1} \mathrm{JCF}_{3}=272.0 \mathrm{~Hz}\right), 119.8,114.9,29.3(\mathrm{q}, J$ (through space) $=6.5 \mathrm{~Hz})$. ${ }^{19} \mathrm{~F} \mathrm{NMR}\left(376 \mathrm{MHz}, \mathrm{CDCl}_{3}, 25^{\circ} \mathrm{C}\right) \delta=-53.90$ (d, J=2.9). HRMS (ESI+): m/z [M+Na] ${ }^{+}$Calcd. for $\mathrm{C}_{10} \mathrm{H}_{6} \mathrm{~F}_{3} \mathrm{NO}_{2} \mathrm{Na:} 252.0243$; Found: 252.0242.

\section{Methyl 1-methyl-2,3-dioxoindoline-7-carboxylate}

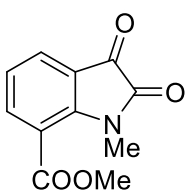

Prepared according to general procedure 1, purified by flash column chromatography (ethyl acetate / $n$-hexane) and obtained as an orange solid (527 mg, 57 \%).

$\mathbf{R}_{\mathbf{f}} 0.32$ (EtOAc / n-hexane, 4:6). M.P. $152-153^{\circ} \mathrm{C} . \mathbf{v}_{\max }$ (neat) $/ \mathrm{cm}^{-1} 3069,3009$, 2955, 1740, 1711, 1589, 1439, 765. ${ }^{1} \mathbf{H}$ NMR $\left(400 \mathrm{MHz}, \mathrm{CDCl}_{3}, 25^{\circ} \mathrm{C}\right) \delta=7.86$ (dd, J=8.0, 1.4, $1 \mathrm{H}, \mathrm{ArH}), 7.73$ (dd, J=7.3, 1.5, 1H, ArH), 7.16 (dd, J=8.0, 7.4, 1H, ArH), $3.97\left(\mathrm{~s}, 3 \mathrm{H}, \mathrm{OCH}_{3}\right.$ ), $3.32\left(\mathrm{~s}, 3 \mathrm{H}, \mathrm{NCH}_{3}\right) .{ }^{13} \mathrm{C} \mathrm{NMR}\left(101 \mathrm{MHz}, \mathrm{CDCl}_{3}, 25^{\circ} \mathrm{C}\right) \delta=182.36,165.94,159.20,150.18$, 139.13, 127.95, 123.39, 119.42, 117.73, 53.03, 30.40. HRMS (ESI+): m/z [M+H] ${ }^{+}$Calcd. for $\mathrm{C}_{11} \mathrm{H}_{10} \mathrm{NO}_{4}: 220.0604$; Found: 220.0608.

\section{1,5,7-trimethylindoline-2,3-dione}

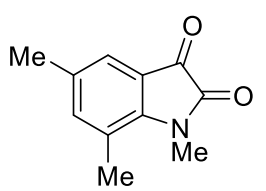

Prepared according to general procedure 1, purified by flash column chromatography (ethyl acetate / $n$-hexane) and obtained as a red solid (443 mg, $68 \%$ ).

$\mathbf{R}_{\mathbf{f}} 0.37$ (EtOAc / $n$-hexane, 4:6). ${ }^{1} \mathbf{H}$ NMR $\left(400 \mathrm{MHz}, \mathrm{CDCl}_{3}, 2^{\circ} \mathrm{C}\right) \delta=7.28-$ $7.26(\mathrm{~m}, 1 \mathrm{H}, \mathrm{ArH}), 7.15-7.13(\mathrm{~m}, 1 \mathrm{H}, \mathrm{ArH}), 3.49\left(\mathrm{~s}, 3 \mathrm{H}, \mathrm{NCH}_{3}\right), 2.52\left(\mathrm{~s}, 3 \mathrm{H}, \mathrm{ArCH}_{3}\right), 2.27$ (s, $\left.3 \mathrm{H}, \mathrm{ArCH}_{3}\right)$.

Data in accordance to literature. ${ }^{7}$

\section{5,7-dibromo-1-methylindoline-2,3-dione}

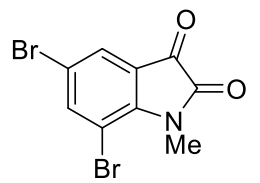

Prepared according to general procedure 1, recrystallized from $\mathrm{CH}_{2} \mathrm{Cl}_{2}$ and obtained as a red solid (416 mg, $66 \%$ ).

$\mathbf{R}_{\mathfrak{f}} 0.5$ (EtOAc / $n$-hexane, 3:7). M.P. 186-188 ${ }^{\circ} \mathrm{C} . \mathbf{v}_{\max }($ neat $) / \mathbf{c m}^{-1} 3069,2949$, $1745,1600,1587,1558,1448 .{ }^{1} \mathrm{H}$ NMR $\left(400 \mathrm{MHz}, \mathrm{CDCl}_{3}, 25^{\circ} \mathrm{C}\right) \delta=7.86(\mathrm{~d}$, $J=2.0,1 \mathrm{H}, \mathrm{ArH}), 7.67$ (d, J=2.0,1H, ArH), $3.64\left(\mathrm{~s}, 3 \mathrm{H}, \mathrm{NCH}_{3}\right) .{ }^{13} \mathrm{C}$ NMR $\left(101 \mathrm{MHz}^{\mathrm{C}} \mathrm{CDCl}_{3}\right.$, $\left.25^{\circ} \mathrm{C}\right) \delta=181.5,158.0,147.4,145.2,127.5,121.1,117.1,105.2,29.9$. HRMS (ESI+): $\mathrm{m} / \mathrm{z}$ $[\mathrm{M}+\mathrm{H}]^{+}$Calcd. for $\mathrm{C}_{9} \mathrm{H}_{6} \mathrm{Br}_{2} \mathrm{NO}_{2}$ : 317.8760; Found: 317.8758 . 


\section{Analytical Data of Hydroxyoxindoles $4 a-v$ and 5-9}

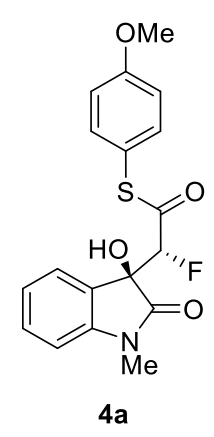

S-(4-methoxyphenyl) (R)-2-fluoro-2-((R)-3-hydroxy-1-methyl-2-oxoindolin3-yl)ethanethioate (4a)

Under inert conditions $(N)$-methylisatin $(161 \mathrm{mg}, 1 \mathrm{mmol}$ ) and catalyst $\mathrm{F}$ (Cat $\mathrm{E}$ in manuscript) (114 mg, $200 \mu \mathrm{mol}, 20 \mathrm{~mol} \%)$ were added to a two-neck flask. Then $20 \mathrm{~mL}$ dry acetone was added and the mixture was cooled to $0^{\circ} \mathrm{C}$. Subsequently, F-MAHT (366 mg, $1.5 \mathrm{mmol}, 1.5$ equiv.) was added as stock solution in $20 \mathrm{~mL}$ dry acetone and the solution ( $25 \mathrm{mM}$ with respect to the isatin) was stirred at $10^{\circ} \mathrm{C}$ for $48 \mathrm{~h}$. After the reaction reached full conversion, the solution was filtered through a plug of silica and evaporated onto celite. The crude product was purified by flash column chromatography ( $5-30 \%$ ethyl acetate / $n$-hexane). (349mg, 98\% yield, 1.5:1 dr).

IR $\mathrm{V}_{\max }$ (neat)/cm $\mathrm{cm}^{-1} 3351,3058,2935,2837,1699,1613,1591,1493,1468,1374,1350,1291$, 1248, 1175, 1106, 1091, 1023, 1001, 952, 827, 782, 752, 725, 693. ${ }^{1} \mathrm{H}$ NMR $\left(400 \mathrm{MHz}, \mathrm{C}_{6} \mathrm{D}_{6}\right.$, $\left.25^{\circ} \mathrm{C}\right) \delta 7.45(\mathrm{dt}, J=7.3,0.7 \mathrm{~Hz}, 1 \mathrm{H}, \mathrm{ArH}), 7.14-7.07(\mathrm{~m}, 2 \mathrm{H}, \mathrm{ArH}), 7.02-6.92(\mathrm{~m}, 1 \mathrm{H}, \mathrm{ArH})$, $6.88-6.78(\mathrm{~m}, 1 \mathrm{H}, \mathrm{ArH}), 6.60-6.53(\mathrm{~m}, 2 \mathrm{H}, \mathrm{ArH}), 6.13-6.09(\mathrm{~m}, 1 \mathrm{H}, \mathrm{ArH}), 5.20(\mathrm{~d}, J=47.8$ $\mathrm{Hz}, 1 \mathrm{H}, \mathrm{C}(\mathrm{F}) \mathrm{H}), 4.24$ (bs, $1 \mathrm{H}, \mathrm{OH}), 3.10\left(\mathrm{~s}, 3 \mathrm{H}, \mathrm{OCH}_{3}\right), 2.48\left(\mathrm{~s}, 3 \mathrm{H}, \mathrm{NCH}_{3}\right)$. Visible peaks of minor diastereoisomers 7.32 (ddt, $J=7.4,1.6,0.8 \mathrm{~Hz}, 1 \mathrm{H}, \mathrm{ArH}), 7.20-7.16(\mathrm{~m}, 2 \mathrm{H}, \mathrm{ArH})$, $6.70-6.62(\mathrm{~m}, 2 \mathrm{H}, \mathrm{ArH}), 5.20(\mathrm{~d}, J=47.8 \mathrm{~Hz}, 1 \mathrm{H}, \mathrm{C}(\mathrm{F}) \mathrm{H}), 4.30$ (bs, $1 \mathrm{H}, \mathrm{OH}), 3.16(\mathrm{~s}, 3 \mathrm{H}$, $\left.\mathrm{OCH}_{3}\right), 2.48\left(\mathrm{~s}, 3 \mathrm{H}, \mathrm{NCH}_{3}\right) .{ }^{13} \mathrm{C}$ NMR $\left(101 \mathrm{MHz}, \mathrm{C}_{6} \mathrm{D}_{6}, 25^{\circ} \mathrm{C}\right) \delta 196.9\left(\mathrm{~d},{ }^{2} \mathrm{~J} \mathrm{C}-\mathrm{F}=29.3 \mathrm{~Hz}\right)$, 173.3, 161.0, 144.7, 136.4, 130.2, 125.8, 125.3, 122.6, 115.9 (d, $\left.{ }^{3} J \mathrm{C}-\mathrm{F}=6.5 \mathrm{~Hz}\right), 114.9,108.3$, $95.5\left(\mathrm{~d},{ }^{1} \mathrm{~J} \mathrm{C}-\mathrm{F}=198.6 \mathrm{~Hz}\right), 76.4\left(\mathrm{~d},{ }^{2} \mathrm{~J} \mathrm{C}-\mathrm{F}=21.2 \mathrm{~Hz}\right), 54.4,25.4$. Visible peaks of minor diastereoisomers: $196.5\left(\mathrm{~d},{ }^{2} \mathrm{~J} \mathrm{C}-\mathrm{F}=27.9 \mathrm{~Hz}\right), 173.1,161.1,144.8,136.2,130.4,125.5,125.2$, 122.4, $116.0\left(\mathrm{~d},{ }^{3} \mathrm{~J} \mathrm{C}-\mathrm{F}=6.8 \mathrm{~Hz}\right), 109.0,94.0\left(\mathrm{~d},{ }^{1} \mathrm{~J} \mathrm{C}-\mathrm{F}=196.3 \mathrm{~Hz}\right), 75.9\left(\mathrm{~d},{ }^{2} \mathrm{~J} \mathrm{C}-\mathrm{F}=24.3\right.$ $\mathrm{Hz}), 56.3,26.4 .{ }^{19} \mathrm{~F}$ NMR $\left(377 \mathrm{MHz}, \mathrm{C}_{6} \mathrm{D}_{6}, 25^{\circ} \mathrm{C}\right) \delta-193.40\left(\mathrm{~d},{ }^{2} \mathrm{~J}_{\mathrm{HF}}=46.3 \mathrm{~Hz}\right)$. Visible peaks of minor diastereoisomers: $199.92\left(\mathrm{~d},{ }^{2} \mathrm{JHF}_{\mathrm{HF}} 47.9 \mathrm{~Hz}\right)$. HRMS (ESI+): $\mathrm{m} / \mathrm{z}[\mathrm{M}+\mathrm{H}]^{+}$Calcd. for $\mathrm{C}_{18} \mathrm{H}_{17} \mathrm{FNO}_{4} \mathrm{~S}$ : 362.0857 ; Found: 362.0849.

\section{S-(4-methoxyphenyl) (R)-2-fluoro-2-((R)-3-hydroxy-2-oxoindolin-3-yl)ethanethioate (4b)}

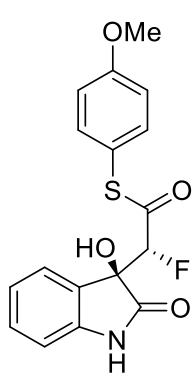

4b

Prepared according to general procedure 2 using catalyst $F$. The product was purified by column chromatography (ethyl acetate / $n$-hexane) to afford the desired product as a white solid $(25.2 \mathrm{mg}, 71 \%$ yield, $1.3: 1 \mathrm{dr})$.

IR $\mathbf{V}_{\max }($ neat $) / \mathrm{cm}^{-1} 3316,2925,2839,2358,1709,1620,1591,1493,1470,1336$, $1292,1249,1177,1106,1024,961,827,754 .{ }^{1} \mathrm{H}$ NMR $\left(400 \mathrm{MHz}, \mathrm{CD}_{2} \mathrm{Cl}_{2}, 25^{\circ} \mathrm{C}\right)$ $\delta 7.72$ (bs, $1 \mathrm{H}, \mathrm{NH}), 7.56-7.49(\mathrm{~m}, 1 \mathrm{H}, \mathrm{ArH}), 7.42-7.34(\mathrm{~m}, 2 \mathrm{H}, \mathrm{ArH}), 7.20-$ $7.11(\mathrm{~m}, 1 \mathrm{H}, \mathrm{ArH}), 7.10-7.05(\mathrm{~m}, 2 \mathrm{H}, \mathrm{ArH}), 6.95-6.85(\mathrm{~m}, 2 \mathrm{H}, \mathrm{ArH}), 5.31(\mathrm{~d}, J$ $=47.7 \mathrm{~Hz}, 1 \mathrm{H}, \mathrm{C}(\mathrm{F}) \mathrm{H}), 3.79\left(\mathrm{~s}, 3 \mathrm{H}, \mathrm{OCH}_{3}\right)$. Visible peaks of minor diastereoisomers: $\delta 7.79$ (bs, $1 \mathrm{H}, \mathrm{NH}), 7.36-7.28(\mathrm{~m}, 2 \mathrm{H}, \mathrm{ArH}), 7.27-7.20(\mathrm{~m}, 1 \mathrm{H}, \mathrm{ArH})$, $7.16-7.08(\mathrm{~m}, 1 \mathrm{H}, \mathrm{ArH}), 7.05-6.97(\mathrm{~m}, 2 \mathrm{H}, \mathrm{ArH}), 6.96-6.87(\mathrm{~m}, 2 \mathrm{H}, \mathrm{ArH}), 5.35(\mathrm{~d}, J=46.1$ $\mathrm{Hz}, 1 \mathrm{H}, \mathrm{C}(\mathrm{F}) \mathrm{H}), 3.85\left(\mathrm{~s}, 3 \mathrm{H}, \mathrm{OCH}_{3}\right) .{ }^{13} \mathrm{C} \mathrm{NMR}\left(101 \mathrm{MHz}, \mathrm{CD}_{2} \mathrm{Cl}_{2}, 25^{\circ} \mathrm{C}\right) \delta 198.2\left(\mathrm{~d},{ }^{2} \mathrm{~J} \mathrm{C}-\mathrm{F}=\right.$ $52.8 \mathrm{~Hz}$ ), 174.8, 161.6, 141.8, 136.7, 131.4, 128.7, 126.0 (d, ${ }^{2} J \mathrm{C}-\mathrm{F}=34.5 \mathrm{~Hz}$ ), 125.8, 123.6, 115.4, 110.9, $95.8\left(\mathrm{~d},{ }^{1} \mathrm{~J} \mathrm{C}-\mathrm{F}=198.5 \mathrm{~Hz}\right), 76.9,55.8$. Visible peaks of minor diastereoisomers: 197.9 (d, ${ }^{1} J$ C-F 54.38 Hz), 174.9, 161.8, 142.0, 131.5, 125.8 (d, ${ }^{2} J$ C-F = 28.8 Hz), 123.5, 115.7, 111.1, $94.2\left(\mathrm{~d},{ }^{1} \mathrm{~J} \mathrm{C}-\mathrm{F}=196.6 \mathrm{~Hz}\right), 76.7,55.9 .{ }^{19} \mathrm{~F}$ NMR $\left(376 \mathrm{MHz}, \mathrm{CD}_{2} \mathrm{Cl}_{2}, 25^{\circ} \mathrm{C}\right) \delta-$ $200.93\left(\mathrm{~d},{ }^{2} \mathrm{JHF}_{\mathrm{HF}}=47.9 \mathrm{~Hz}\right)$. Visible peaks of minor diastereoisomers: $\delta-194.76\left(\mathrm{~d},{ }^{2} \mathrm{JHF}_{\mathrm{HF}}=46.3\right.$ Hz). HRMS (ESI+): m/z [M+Na] ${ }^{+}$Calcd. for $\mathrm{C}_{17} \mathrm{H}_{14} \mathrm{FNO}_{4} \mathrm{SNa}$ : 370.0520; Found: 370.0516 . 


\section{S-(4-methoxyphenyl) (R)-2-fluoro-2-((R)-3-hydroxy-1,5-dimethyl-2-oxoindolin-3- yl)ethanethioate (4c)}

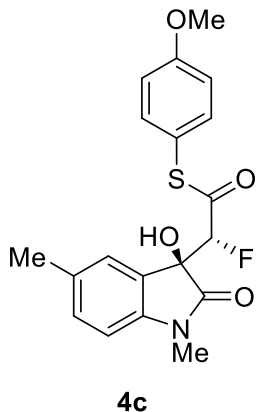

Prepared according to general procedure 2 using catalyst $F$. The product was purified by column chromatography (ethyl acetate / $n$-hexane) to afford the desired product as a white solid (37.6 $\mathrm{mg}, 98 \%$ yield, 2.2:1 dr).

IR $\mathrm{v}_{\max }\left(\right.$ neat) $/ \mathrm{cm}^{-1} 3365,2924,2851,1701,1622,1592,1495,1463,1362$, $1291,1250,1175,1106,1027,827,740 .{ }^{1} \mathrm{H}$ NMR $\left(400 \mathrm{MHz}, \mathrm{C}_{6} \mathrm{D}_{6}, 25^{\circ} \mathrm{C}\right) \delta$ $7.41-7.36(\mathrm{~m}, 1 \mathrm{H}, \mathrm{ArH}), 7.14-7.09(\mathrm{~m}, 2 \mathrm{H}, \mathrm{ArH}), 6.84(\mathrm{~d}, J=7.9 \mathrm{~Hz}, 1 \mathrm{H}$, ArH), $6.62-6.54(\mathrm{~m}, 2 \mathrm{H}, \mathrm{ArH}), 6.13(\mathrm{~d}, J=7.8 \mathrm{~Hz}, 1 \mathrm{H}, \mathrm{ArH}), 5.45-5.26(\mathrm{~m}$, $1 \mathrm{H}, \mathrm{C}(\mathrm{F}) \mathrm{H}), 4.69(\mathrm{bs}, 1 \mathrm{H}, \mathrm{OH}), 3.12\left(\mathrm{~s}, 3 \mathrm{H}, \mathrm{OCH}_{3}\right), 2.54\left(\mathrm{~s}, 3 \mathrm{H}, \mathrm{NCH}_{3}\right), 2.08$ (s, $\left.3 \mathrm{H}, \mathrm{ArCH}_{3}\right)$. Visible peaks of minor diastereoisomer: $7.25-7.18(\mathrm{~m}, 2 \mathrm{H}, \mathrm{ArH}), 6.84(\mathrm{~d}, \mathrm{~J}=$ $7.9 \mathrm{~Hz}, 1 \mathrm{H}, \mathrm{ArH}), 6.72-6.64(\mathrm{~m}, 2 \mathrm{H}, \mathrm{ArH}), 5.56-5.50(\mathrm{~m}, 1 \mathrm{H}, \mathrm{C}(\mathrm{F}) \mathrm{H}), 3.19(\mathrm{~d}, J=0.8 \mathrm{~Hz}$, $\left.3 \mathrm{H}, \mathrm{OCH}_{3}\right), 2.54\left(\mathrm{~s}, 3 \mathrm{H}, \mathrm{NCH}_{3}\right) .{ }^{13} \mathrm{C}$ NMR $\left(101 \mathrm{MHz}, \mathrm{C}_{6} \mathrm{D}_{6}, 2^{\circ} \mathrm{C}\right) \delta 197.2\left(\mathrm{~d},{ }^{2} \mathrm{~J} \mathrm{C}-\mathrm{F}=32.6 \mathrm{~Hz}\right)$, 173.9, 161.3, 142.7, 136.8, 132.6, 130.9, 126.7, 126.3, 116.4, 116.4, 115.3, 108.5, 96.1 (d, ${ }^{1} \mathrm{~J}$ $\mathrm{C}-\mathrm{F}=198.8 \mathrm{~Hz}), 77.0\left(\mathrm{~d},{ }^{2} \mathrm{~J} \mathrm{C}-\mathrm{F}=21.0 \mathrm{~Hz}\right), 54.7,25.9,21.0$. Visible peaks of minor diastereoisomer: 196.7, 161.5, 142.8, 136.7, 132.3, 131.0, 125.9, 116.5, 115.4, 108.7, 94.6 (d, $\left.{ }^{1} \mathrm{~J} \mathrm{C}-\mathrm{F}=196.2 \mathrm{~Hz}\right), 76.5\left(\mathrm{~d},{ }^{2} \mathrm{~J} \mathrm{C}-\mathrm{F}=24.45 \mathrm{~Hz}\right), 54.9,25.9,21.0 .{ }^{19} \mathrm{~F}$ NMR $\left(377 \mathrm{MHz}, \mathrm{C}_{6} \mathrm{D}_{6}\right.$, $\left.25^{\circ} \mathrm{C}\right) \delta-193.10--193.36(\mathrm{~m})$. Visible peaks of minor diastereoisomer: $-199.70--200.14(\mathrm{~m})$. HRMS (ESI+): m/z [M+Na] ${ }^{+}$Calcd. for $\mathrm{C}_{19} \mathrm{H}_{18} \mathrm{FNO}_{4} \mathrm{SNa}$ : 398.0833; Found:398.0827.

\section{S-(4-methoxyphenyl) (R)-2-fluoro-2-((R)-3-hydroxy-5-methoxy-1-methyl-2-oxoindolin-3- yl)ethanethioate (4d)}

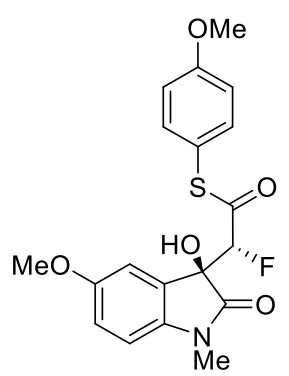

4d

Prepared according to general procedure 2 using catalyst $F$. The product was purified by column chromatography (ethyl acetate / $n$-hexane) to afford the desired product as a white solid $(24.9 \mathrm{mg}, 78 \%$ yield, $2.2: 1 \mathrm{dr})$.

IR $\mathbf{v}_{\max }$ (neat) $/ \mathrm{cm}^{-1} 3343,2938,2836,1694,1592,1493,1471,1437,1365$, $1288,1248,1173,1108,1095,1026,1005,960,883,827,802,758,739$, 707, 669. ${ }^{1} \mathrm{H}$ NMR $\left(400 \mathrm{MHz}, \mathrm{C}_{6} \mathrm{D}_{6}, 25^{\circ} \mathrm{C}\right) \delta=7.25(\mathrm{~d}, \mathrm{~J}=2.6,1 \mathrm{H}, \mathrm{ArH}), 7.18$ (d, 2H, ArH), 6.70 (dd, J=8.5, 2.6, 1H, ArH), 6.58 (d, J=8.8, 2H, ArH), 6.08 (d, J=8.5, 1H, ArH), $5.18\left(\mathrm{~d},{ }^{2} J_{\mathrm{HF}}=47.7,1 \mathrm{H}, \mathrm{C}(\mathrm{F}) \mathrm{H}\right), 4.38(\mathrm{~s}, 1 \mathrm{H}, \mathrm{OH}), 3.32$ $\left(\mathrm{s}, 3 \mathrm{H}, \mathrm{OCH}_{3}\right), 3.11\left(\mathrm{~s}, 3 \mathrm{H}, \mathrm{OCH}_{3}\right), 2.52\left(\mathrm{~s}, 3 \mathrm{H}, \mathrm{NCH}_{3}\right)$. Visible peaks of minor diastereoisomer: $7.21(\mathrm{~d}, J=8.8,2 \mathrm{H}, \mathrm{ArH}), 7.14(\mathrm{~d}, \mathrm{~J}=2.6,1 \mathrm{H}, \mathrm{ArH}), 6.64(\mathrm{~d}, \mathrm{~J}=8.7,2 \mathrm{H}, \mathrm{ArH}), 6.10(\mathrm{~d}, J=8.4,1 \mathrm{H}$, ArH), $5.40\left(\mathrm{~d},{ }^{2} \mathrm{~J}_{\mathrm{HF}}=46.0,1 \mathrm{H}, \mathrm{C}(\mathrm{F}) \mathrm{H}\right), 3.30\left(\mathrm{~s}, 3 \mathrm{H}, \mathrm{OCH}_{3}\right), 3.16\left(\mathrm{~s}, 3 \mathrm{H}, \mathrm{OCH}_{3}\right), 2.53(\mathrm{~s}, 3 \mathrm{H}$, $\left.\mathrm{NCH}_{3}\right) .{ }^{13} \mathrm{C}$ NMR $\left(101 \mathrm{MHz}, \mathrm{C}_{6} \mathrm{D}_{6}, 25^{\circ} \mathrm{C}\right) \delta=197.3\left(\mathrm{~d},{ }^{2} \mathrm{~J} \mathrm{C}-\mathrm{F}=29.5\right), 173.5\left(\mathrm{~d},{ }^{3} \mathrm{~J} \mathrm{C}-\mathrm{F}=4.2\right)$, 161.4, 156.8, 138.3, 136.8, 127.2, 116.3 (d, $\left.{ }^{3} J \mathrm{C}-\mathrm{F}=6.6\right), 116.0,115.3,112.2,109.3,95.8$ (d, $\left.{ }^{1} \mathrm{~J} \mathrm{C}-\mathrm{F}=198.5\right), 77.2\left(\mathrm{~d},{ }^{2} \mathrm{~J} \mathrm{C}-\mathrm{F}=21.2\right), 55.4,54.7,25.9$. Visible peaks of minor diastereoisomer: 161.5, 156.5, 138.5, 136.6, 127.0, 115.4, 112.9, 109.3, 94.5 (d, ${ }^{1} \mathrm{~J} C-F=$ 196.5), 55.4, 54.8, 26.0. ${ }^{19} \mathrm{~F}$ NMR $\left(376 \mathrm{MHz}, \mathrm{C}_{6} \mathrm{D}_{6}, 25^{\circ} \mathrm{C}\right) \delta-199.66\left(\mathrm{~d},{ }^{2} \mathrm{~J}_{\mathrm{HF}}=47.8 \mathrm{~Hz}\right)$. Visible peaks of minor diastereoisomer: $-193.43\left(\mathrm{~d},{ }^{2} \mathrm{JHF}_{\mathrm{HF}}=45.9\right)$. HRMS (ESI+): $\mathrm{m} / \mathrm{z}[\mathrm{M}+\mathrm{H}]^{+}$Calcd. for $\mathrm{C}_{19} \mathrm{H}_{19} \mathrm{FNO}_{5} \mathrm{~S}$ : 392.0962 ; Found: 392.0964. 


\section{S-(4-methoxyphenyl) (R)-2-fluoro-2-((R)-3-hydroxy-5-methoxy-2-oxoindolin-3-}

\section{yl)ethanethioate $(4 e)$}<smiles>COc1ccc(NC(=O)C(F)(F)C(F)(F)C(=O)Sc2ccc(OC)cc2)cc1</smiles>

Prepared according to general procedure 2 using catalyst $F$. The product was purified by column chromatography (ethyl acetate / $n$-hexane) to afford the desired product as a white solid $(20.0 \mathrm{mg}, 52 \%$ yield, $1.4: 1 \mathrm{dr})$.

IR $\mathbf{v}_{\max }$ (neat) $/ \mathrm{cm}^{-1} 3305,3170,3088,2926,2838,2359,1721,1698,1592$, 1492, 1358, 1279, 1250, 1211, 1174, 1161, 1029, 827. 'H NMR $(400 \mathrm{MHz}$, $\left.\mathrm{CD}_{2} \mathrm{Cl}_{2}, 25^{\circ} \mathrm{C}\right) \delta 7.65(\mathrm{~s}, 1 \mathrm{H}, \mathrm{NH}), 7.36-7.29(\mathrm{~m}, 2 \mathrm{H}, \mathrm{ArH}), 7.05-6.97(\mathrm{~m}$, $2 \mathrm{H}, \mathrm{ArH}), 6.94-6.81(\mathrm{~m}, 3 \mathrm{H}, \mathrm{ArH}), 5.33(\mathrm{~d}, J=46.1 \mathrm{~Hz}, 1 \mathrm{H}, \mathrm{C}(\mathrm{F}) \mathrm{H}), 4.06$ $(\mathrm{s}, 1 \mathrm{H}, \mathrm{OH}), 3.85\left(\mathrm{~s}, 3 \mathrm{H}, \mathrm{OCH}_{3}\right), 3.78\left(\mathrm{~s}, 3 \mathrm{H}, \mathrm{OCH}_{3}\right) .{ }^{13} \mathrm{C}$ NMR $(101 \mathrm{MHz}$, $\left.\mathrm{CD}_{2} \mathrm{Cl}_{2}, 25^{\circ} \mathrm{C}\right) \delta 198.3\left(\mathrm{~d},{ }^{2} \mathrm{~J} \mathrm{C}-\mathrm{F}=28.1 \mathrm{~Hz}\right), 174.6,161.8,156.6,136.7,135.1,126.8,116.0$, 115.7, 112.8, 111.5, $94.2\left(\mathrm{~d},{ }^{1} J \mathrm{C}-\mathrm{F}=196.8 \mathrm{~Hz}\right), 76.6\left(\mathrm{~d},{ }^{1} J \mathrm{C}-\mathrm{F}=24.0 \mathrm{~Hz}\right), 56.2,55.9 .{ }^{19} \mathrm{~F}$ NMR $\left(376 \mathrm{MHz}, \mathrm{CD}_{2} \mathrm{Cl}_{2}, 25^{\circ} \mathrm{C}\right) \delta-194.88\left(\mathrm{~d},{ }^{2} J_{\mathrm{HF}}=46.1 \mathrm{~Hz}\right)$. HRMS (ESI+): $\mathrm{m} / \mathrm{z}[\mathrm{M}+\mathrm{Na}]^{+}$ Calcd. for $\mathrm{C}_{18} \mathrm{H}_{16} \mathrm{FNO}_{5} \mathrm{SNa}$ : 400.0625 ; Found: 400.0625.

\section{S-(4-methoxyphenyl) (R)-2-fluoro-2-((R)-5-fluoro-3-hydroxy-1-methyl-2-oxoindolin-3- yl)ethanethioate (4f)}

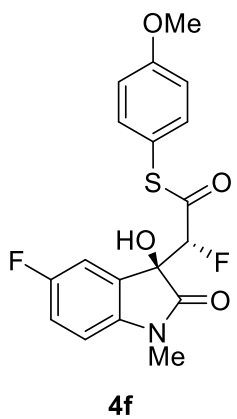

Prepared according to general procedure 2 using catalyst $F$. The product was purified by column chromatography (ethyl acetate / $n$-hexane) to afford the desired product as a white solid $(27.0 \mathrm{mg}, 87 \%$ yield, $2.0: 1 \mathrm{dr})$.

IR $\mathrm{V}_{\max }\left(\right.$ neat) $/ \mathrm{cm}^{-1} 3343.9,2939.3,2838.8,2359.7,1700.4,1623.9,1492.8$, 1467.4, 1365.9, 1291.1, 1249.4, 1174.1, 1107.2, 1026.9. ${ }^{1} \mathbf{H}$ NMR $(400 \mathrm{MHz}$, $\left.\mathrm{C}_{6} \mathrm{D}_{6}, 25^{\circ} \mathrm{C}\right) \delta=7.25(\mathrm{dd}, \mathrm{J}=7.5,2.7,1 \mathrm{H}, \mathrm{ArH}), 7.15(\mathrm{~d}, \mathrm{~J}=8.9,2 \mathrm{H}, \mathrm{ArH}), 6.76$ $-6.60(\mathrm{~m}, 1 \mathrm{H}, \mathrm{ArH}), 6.58(\mathrm{~d}, J=8.8,2 \mathrm{H}, \mathrm{ArH}), 5.81(\mathrm{dd}, J=8.6,4.0,1 \mathrm{H}, \mathrm{ArH})$, $5.08\left(\mathrm{~d},{ }^{2} \mathrm{JHF}_{\mathrm{HF}}=47.5,1 \mathrm{H}, \mathrm{C}(\mathrm{F}) \mathrm{H}\right), 4.37(\mathrm{~s}, 1 \mathrm{H}, \mathrm{OH}), 3.11\left(\mathrm{~s}, 3 \mathrm{H}, \mathrm{OCH}_{3}\right), 2.42(\mathrm{~s}$, $\left.3 \mathrm{H}, \mathrm{NCH}_{3}\right)$. Visible peaks of minor diastereoisomer: $5.31\left(\mathrm{~d},{ }^{2} \mathrm{~J}_{\mathrm{HF}}=46.0,1 \mathrm{H}, \mathrm{C}(\mathrm{F}) \mathrm{H}\right), 3.16(\mathrm{~s}$, $\left.3 \mathrm{H}, \mathrm{OCH}_{3}\right), 2.43\left(\mathrm{~s}, 3 \mathrm{H}, \mathrm{NCH}_{3}\right) .{ }^{13} \mathrm{C}$ NMR $\left(101 \mathrm{MHz}, \mathrm{C}_{6} \mathrm{D}_{6}, 25^{\circ} \mathrm{C}\right) \delta=197.3\left(\mathrm{~d},{ }^{2} J \mathrm{C}-\mathrm{F}=29.1\right)$, 173.5, 161.4, $159.6\left(\mathrm{~d},{ }^{1} J \mathrm{C}-\mathrm{F}=241.7\right), 140.9\left(\mathrm{~d},{ }^{4} J \mathrm{C}-\mathrm{F}=2.1\right), 136.7,127.6\left(\mathrm{~d},{ }^{3} J \mathrm{C}-\mathrm{F}=8.1\right)$, $116.9\left(\mathrm{~d},{ }^{2} \mathrm{~J} \mathrm{C}-\mathrm{F}=23.5\right), 116.1\left(\mathrm{~d},{ }^{3} J \mathrm{C}-\mathrm{F}=6.4\right), 115.3,113.7\left(\mathrm{~d},{ }^{2} J \mathrm{C}-\mathrm{F}=24.9\right), 109.3\left(\mathrm{~d},{ }^{3} \mathrm{~J}\right.$ $\mathrm{C}-\mathrm{F}=7.8), 95.4\left(\mathrm{~d},{ }^{1} \mathrm{~J} \mathrm{C}-\mathrm{F}=198.8\right), 76.9\left(\mathrm{dd},{ }^{2} \mathrm{~J} \mathrm{C}-\mathrm{F}=21.3,1.6\right), 54.8,25.9$. Visible peaks of minor diastereoisomer: 161.6, 136.6, $117.0\left(\mathrm{~d},{ }^{2} \mathrm{~J} \mathrm{C}-\mathrm{F}=23.2\right), 115.5,113.8\left(\mathrm{~d},{ }^{2} \mathrm{~J} \mathrm{C}-\mathrm{F}=25.3\right)$, 109.5 (d, $\left.{ }^{3} \mathrm{~J} \mathrm{C}-\mathrm{F}=7.9\right), 94.1\left(\mathrm{~d},{ }^{1} \mathrm{~J} \mathrm{C}-\mathrm{F}=196.6\right), 54.9,25.9 .{ }^{19} \mathrm{~F} \mathrm{NMR}\left(376 \mathrm{MHz}, \mathrm{C}_{6} \mathrm{D}_{6}, 25^{\circ} \mathrm{C}\right)$ $\delta=-109.91--124.11(\mathrm{~m}),-200.03\left(\mathrm{~d},{ }^{2} J_{\mathrm{HF}}=47.3\right)$. Visible peaks of minor diastereoisomer: $109.91--124.11(\mathrm{~m}),-193.80\left(\mathrm{~d},{ }^{2} J_{\mathrm{HF}}=45.8\right)$. HRMS (ESI+): $\mathrm{m} / \mathrm{z}[\mathrm{M}+\mathrm{H}]^{+}$Calcd. for $\mathrm{C}_{18} \mathrm{H}_{16} \mathrm{~F}_{2} \mathrm{NO}_{4} \mathrm{~S}: 380.0763$; Found: 380.0767. 


\section{S-(4-methoxyphenyl) (R)-2-((R)-5-chloro-3-hydroxy-1-methyl-2-oxoindolin-3-yl)-2- fluoroethanethioate $(\mathbf{4 g})$}

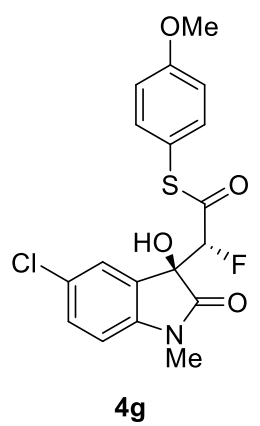

Prepared according to general procedure 2 using catalyst $F$. The product was purified by column chromatography (ethyl acetate / $n$-hexane) to afford the desired product as a white solid (30.0 mg, 93\% yield, 1.9:1 dr).

IR $\mathrm{V}_{\max }$ (neat) $/ \mathrm{cm}^{-1} 3368.0,2928.5,2840.0,2358.9,2336.7,1713.4,1612.5$, 1592.0, 1492.5, 1463.2, 1362.5, 1250.6, 1105.4, 1028.3. ${ }^{1} \mathrm{H}$ NMR $(400 \mathrm{MHz}$, $\left.\mathrm{C}_{6} \mathrm{D}_{6}, 25^{\circ} \mathrm{C}\right) \delta=7.49(\mathrm{~d}, \mathrm{~J}=2.1,1 \mathrm{H}, \mathrm{ArH}), 7.18(\mathrm{~d}, 2 \mathrm{H}, \mathrm{ArH}), 6.99-6.93(\mathrm{~m}$, $1 \mathrm{H}, \operatorname{ArH}), 6.58(\mathrm{~d}, \mathrm{~J}=8.8,2 \mathrm{H}, \operatorname{ArH}), 5.78(\mathrm{~d}, \mathrm{~J}=8.3,1 \mathrm{H}, \operatorname{ArH}), 4.98(\mathrm{~d}$,

$\left.{ }^{2} J_{\mathrm{HF}}=47.4,1 \mathrm{H}, \mathrm{C}(\mathrm{F}) \mathrm{H}\right), 4.22(\mathrm{~s}, 1 \mathrm{H}, \mathrm{OH}), 3.11\left(\mathrm{~s}, 3 \mathrm{H}, \mathrm{OCH}_{3}\right), 2.37(\mathrm{~s}, 3 \mathrm{H}$, $\left.\mathrm{NCH}_{3}\right)$. Visible peaks of minor diastereoisomer: $7.36(\mathrm{~d}, \mathrm{~J}=2.1,1 \mathrm{H}, \mathrm{ArH}), 7.24(\mathrm{~d}, \mathrm{~J}=8.8,2 \mathrm{H}$, $\operatorname{ArH}), 6.69$ (d, J=8.8, 2H, ArH), 5.79 (d, J=8.3, 1H, ArH), 5.25 (d, $\left.{ }^{2} J_{H F}=46.1,1 \mathrm{H}, \mathrm{C}(\mathrm{F}) \mathrm{H}\right), 4.15$ $(\mathrm{s}, 1 \mathrm{H}, \mathrm{OH}), 3.16\left(\mathrm{~s}, 3 \mathrm{H}, \mathrm{OCH}_{3}\right), 2.38\left(\mathrm{~s}, 3 \mathrm{H}, \mathrm{NCH}_{3}\right) .{ }^{13} \mathrm{C} \mathrm{NMR}\left(101 \mathrm{MHz}, \mathrm{C}_{6} \mathrm{D}_{6}, 25^{\circ} \mathrm{C}\right) \delta=$ $197.6\left(d,{ }^{2} J \mathrm{C}-\mathrm{F}=29.4\right), 173.1\left(\mathrm{~d},{ }^{3} J \mathrm{C}-\mathrm{F}=3.8\right), 161.5,143.5,136.8,130.6,126.0,116.0(\mathrm{~d}$, $\left.{ }^{3} \mathrm{~J} \mathrm{C}-\mathrm{F}=6.6\right), 115.4,109.7,95.1\left(\mathrm{~d},{ }^{1} \mathrm{~J} \mathrm{C}-\mathrm{F}=198.6\right), 76.6\left(\mathrm{~d},{ }^{2} \mathrm{~J} \mathrm{C}-\mathrm{F}=21.6\right), 54.8,25.8$. Visible peaks of minor diastereoisomer: 161.7, 143.7, 130.7, 126.1, 115.6, 109.9, 94.0 (d, ${ }^{\mathrm{J}} \mathrm{C}-\mathrm{F}=$ 196.8), 54.9, 25.9. ${ }^{19} \mathrm{~F}$ NMR $\left(376 \mathrm{MHz}, \mathrm{C}_{6} \mathrm{D}_{6}, 25^{\circ} \mathrm{C}\right) \delta=-199.32\left(\mathrm{~d},{ }^{2} \mathrm{JHF}_{\mathrm{HF}}=47.3\right)$. Visible peaks of minor diastereoisomer: -194.01 (d, $\left.{ }^{2} \mathrm{JHF}_{\mathrm{HF}} 46.0\right)$. HRMS (ESI+): $\mathrm{m} / \mathrm{z}[\mathrm{M}+\mathrm{Na}]^{+}$Calcd. for $\mathrm{C}_{18} \mathrm{H}_{15} \mathrm{CIFNO}_{4} \mathrm{SNa}: 418.0287$; Found: 418.0290.

\section{S-(4-methoxyphenyl) (R)-2-((R)-5-bromo-3-hydroxy-1-methyl-2-oxoindolin-3-yl)-2-}

\section{fluoroethanethioate $(4 \mathrm{~h})$}

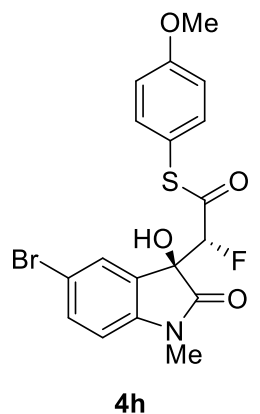

Prepared according to general procedure 2 using catalyst $F$. The product was purified by column chromatography (ethyl acetate / $n$-hexane) to afford the desired product as a white solid $(30.0 \mathrm{mg}, 83 \%$ yield, $1.7: 1 \mathrm{dr})$.

IR $v_{\max }$ (neat) $/ \mathrm{cm}^{-1} 3366,2937,2838.5,1715 ; 1610,1592,1492,1462,1360$, 1251, 1175, 1105, 1028. ${ }^{1} \mathrm{H}$ NMR $\left(400 \mathrm{MHz}, \mathrm{C}_{6} \mathrm{D}_{6}, 25^{\circ} \mathrm{C}\right) \delta=7.67(\mathrm{~d}, \mathrm{~J}=2.0$, $1 \mathrm{H}, \mathrm{ArH}), 7.15(\mathrm{~d}, \mathrm{~J}=8.8,2 \mathrm{H}, \operatorname{ArH}), 7.12(\mathrm{~d}, J=2.0,1 \mathrm{H}, \operatorname{ArH}), 6.58(\mathrm{~d}, J=8.8$, $2 \mathrm{H}, \mathrm{ArH}), 5.74(\mathrm{~d}, \mathrm{~J}=8.3,1 \mathrm{H}, \mathrm{ArH}), 5.03\left(\mathrm{~d},{ }^{2} \mathrm{JHF}_{\mathrm{H}}=47.5,1 \mathrm{H}, \mathrm{C}(\mathrm{F}) \mathrm{H}\right), 4.37(\mathrm{~s}$, $1 \mathrm{H}, \mathrm{OH}), 3.11\left(\mathrm{~s}, 3 \mathrm{H}, \mathrm{OCH}_{3}\right), 2.37\left(\mathrm{~s}, 3 \mathrm{H}, \mathrm{NCH}_{3}\right)$. Visible peaks of minor diastereoisomer: $7.51(\mathrm{~d}, \mathrm{~J}=2.0,1 \mathrm{H}, \mathrm{ArH}), 7.27(\mathrm{~d}, \mathrm{~J}=8.7,2 \mathrm{H}, \mathrm{ArH}), 7.10(\mathrm{~d}, \mathrm{~J}=2.0,1 \mathrm{H}, \mathrm{ArH})$, $6.71(\mathrm{~d}, \mathrm{~J}=8.7,2 \mathrm{H}, \mathrm{ArH}), 5.28\left(\mathrm{~d},{ }^{2} \mathrm{JHF}_{\mathrm{HF}}=46.1,1 \mathrm{H}, \mathrm{C}(\mathrm{F}) \mathrm{H}\right), 3.18\left(\mathrm{~s}, 3 \mathrm{H}, \mathrm{OCH}_{3}\right), 2.38(\mathrm{~s}, 3 \mathrm{H}$, $\left.\mathrm{NCH}_{3}\right) .{ }^{13} \mathrm{C}$ NMR $\left(101 \mathrm{MHz}, \mathrm{C}_{6} \mathrm{D}_{6}, 25^{\circ} \mathrm{C}\right) \delta=197.5$ (d, $\left.{ }^{2} \mathrm{~J} \mathrm{C}-\mathrm{F}=28.9\right), 173.1$ (d, $\left.{ }^{4} \mathrm{~J} \mathrm{C}-\mathrm{F}=4.2\right)$, $161.4,144.0,136.7,133.5,128.7,116.0\left(\mathrm{~d},{ }^{3} J \mathrm{C}-\mathrm{F}=6.4\right), 115.6\left(\mathrm{~d},{ }^{3} J \mathrm{C}-\mathrm{F}=8.9\right), 115.5,115.4$, 110.2, $95.2\left(\mathrm{~d},{ }^{1} \mathrm{~J} \mathrm{C}-\mathrm{F}=198.6\right), 76.6\left(\mathrm{~d},{ }^{2} \mathrm{~J} \mathrm{C}-\mathrm{F}=21.4\right), 54.8,25.8$. Visible peaks of minor diastereoisomer: $161.7,144.1,136.8,133.6,128.8,115.8\left(d,{ }^{2} J \mathrm{C}-\mathrm{F}=5.7\right), 110.4,94.1\left(\mathrm{~d},{ }^{1} \mathrm{~J}\right.$ $\mathrm{C}-\mathrm{F}=196.5), 54.9,25.9 .{ }^{19} \mathrm{~F}$ NMR $\left(376 \mathrm{MHz}, \mathrm{C}_{6} \mathrm{D}_{6}\right) \delta=-199.47\left(\mathrm{~d},{ }^{2} J_{\mathrm{HF}}=47.4\right)$. Visible peaks of minor diastereoisomer: $-193.95\left(\mathrm{~d},{ }^{2} \mathrm{~J}_{\mathrm{HF}}=45.9\right)$. HRMS (ESI+): $\mathrm{m} / \mathrm{z}[\mathrm{M}+\mathrm{Na}]^{+}$Calcd. for $\mathrm{C}_{18} \mathrm{H}_{15} \mathrm{BrFNO}_{4} \mathrm{SNa}: 461.9781$; Found: 461.9778. 


\section{S-(4-methoxyphenyl) (R)-2-fluoro-2-(( $R)$-4-fluoro-3-hydroxy-1-methyl-2-oxoindolin-3- yl)ethanethioate (4i)}

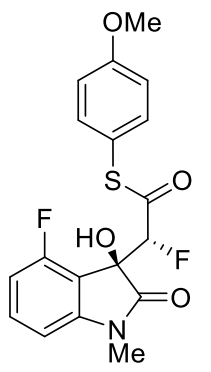

$4 \mathbf{i}$

Prepared according to general procedure 2 using catalyst $F$. The product was purified by column chromatography (ethyl acetate / $n$-hexane) to afford the desired product as a white solid (38.0 mg, 98\% yield, $1.8: 1 \mathrm{dr}$ ).

IR $\mathrm{v}_{\max }$ (neat)/ $/ \mathrm{cm}^{-1} 3357,3070,2928,2839,1713,1627,1591,1493,1471,1349$, 1291, 1243, 1175, 1149, 1091, 1064, 1019, 827, 789, 732. ' H NMR $(400 \mathrm{MHz}$, $\left.\mathrm{C}_{6} \mathrm{D}_{6}, 25^{\circ} \mathrm{C}\right) \delta 7.31-7.22(\mathrm{~m}, 2 \mathrm{H}, \mathrm{ArH}), 6.79-6.69(\mathrm{~m}, 1 \mathrm{H}, \mathrm{ArH}), 6.73-6.59$ $(\mathrm{m}, 2 \mathrm{H}, \mathrm{ArH}), 6.52-6.43(\mathrm{~m}, 1 \mathrm{H}, \mathrm{ArH}), 5.86-5.77(\mathrm{~m}, 1 \mathrm{H}, \mathrm{ArH}), 5.43(\mathrm{~d}, J=$ $47.3 \mathrm{~Hz}, 1 \mathrm{H}, \mathrm{C}(\mathrm{F}) \mathrm{H}), 4.68(\mathrm{~d}, J=4.1 \mathrm{~Hz}, 1 \mathrm{H}, \mathrm{OH}), 3.14\left(\mathrm{~s}, 3 \mathrm{H}, \mathrm{OCH}_{3}\right), 2.41$ (s, $3 \mathrm{H}, \mathrm{NH}_{3}$ ). Visible peaks of minor diastereoisomers: $5.52(\mathrm{dt}, J=46.0,1.2 \mathrm{~Hz}, 1 \mathrm{H}, \mathrm{C}(\mathrm{F}) \mathrm{H}), 3.16$ $\left(\mathrm{s}, 3 \mathrm{H}, \mathrm{OCH}_{3}\right), 2.44\left(\mathrm{~s}, 3 \mathrm{H}, \mathrm{NCH}_{3}\right) \cdot{ }^{13} \mathrm{C}$ NMR $\left(101 \mathrm{MHz}, \mathrm{C}_{6} \mathrm{D}_{6}, 25^{\circ} \mathrm{C}\right) \delta 198.4\left(\mathrm{~d},{ }^{2} \mathrm{~J} \mathrm{C}-\mathrm{F}=29.4\right.$ $\mathrm{Hz}$ ), 172.9, 161.5, $161.4\left(\mathrm{~d},{ }^{2} \mathrm{~J} \mathrm{C}-\mathrm{F}=22.7 \mathrm{~Hz}\right), 159.0,147.1,136.8,132.8\left(\mathrm{~d},{ }^{3} \mathrm{~J}-\mathrm{F}=2.6 \mathrm{~Hz}\right)$, 116.2, 116.1, 115.4, $112.0\left(\mathrm{~d},{ }^{2} \mathrm{~J} \mathrm{C}-\mathrm{F}=19.4 \mathrm{~Hz}\right), 110.9\left(\mathrm{~d},{ }^{2} \mathrm{~J} \mathrm{C}-\mathrm{F}=20.8 \mathrm{~Hz}\right), 104.9$ (d, ${ }^{3} \mathrm{~J} \mathrm{C}-\mathrm{F}$ $=3.2 \mathrm{~Hz}), 93.8\left(\mathrm{~d},{ }^{1} \mathrm{~J} \mathrm{C}-\mathrm{F}=196.9 \mathrm{~Hz}\right), 77.2\left(\mathrm{~d},{ }^{2} \mathrm{~J} \mathrm{C}-\mathrm{F}=23.0 \mathrm{~Hz}\right), 54.8,26.1$. Visible peaks of minor diastereoisomers: $\delta 196.9\left(\mathrm{~d},{ }^{2} \mathrm{~J} \mathrm{C}-\mathrm{F}=27.0 \mathrm{~Hz}\right), 172.8,161.3,158.8,147.0,136.7\left(\mathrm{~d},{ }^{3} \mathrm{~J}\right.$ $\mathrm{C}-\mathrm{F}=1.4 \mathrm{~Hz}), 132.7,132.70,116.2,116.1,111.9\left(\mathrm{~d},{ }^{2} \mathrm{~J} \mathrm{C}-\mathrm{F}=16.9 \mathrm{~Hz}\right), 110.7\left(\mathrm{~d},{ }^{2} \mathrm{~J}-\mathrm{F}=21.2\right.$ $\mathrm{Hz}$ ), $105.0\left(\mathrm{~d},{ }^{3} \mathrm{~J} \mathrm{C}-\mathrm{F}=3.2 \mathrm{~Hz}\right), 93.6\left(\mathrm{~d},{ }^{1} \mathrm{~J} \mathrm{C}-\mathrm{F}=198.2 \mathrm{~Hz}\right), 76.8\left(\mathrm{~d},{ }^{1} \mathrm{~J} \mathrm{C}-\mathrm{F}=24.7 \mathrm{~Hz}\right), 54.8$, 26.2. ${ }^{19} \mathrm{~F}$ NMR $\left(377 \mathrm{MHz}, \mathrm{C}_{6} \mathrm{D}_{6}, 25^{\circ} \mathrm{C}\right) \delta-113.66,-194.72\left(\mathrm{~d},{ }^{2} \mathrm{~J}_{\mathrm{HF}}=46.3 \mathrm{~Hz}\right)$. Visible peaks of minor diastereoisomers: -116.34, -195.93 - -196.23 (m). HRMS (ESI+): m/z [M+Na] ${ }^{+}$Calcd. for $\mathrm{C}_{18} \mathrm{H}_{15} \mathrm{~F}_{2} \mathrm{NO}_{4} \mathrm{SNa}$ : 402.0582; Found: 402.0584.

\section{S-(4-methoxyphenyl) (R)-2-((R)-4-chloro-3-hydroxy-1-methyl-2-oxoindolin-3-yl)-2-}

\section{fluoroethanethioate (4j)}

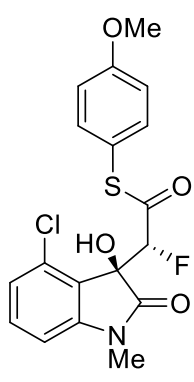

$4 \mathbf{j}$

Prepared according to general procedure 2 using catalyst $F$. The product was purified by column chromatography (ethyl acetate / $n$-hexane) to afford the desired product as a white solid $(29.1 \mathrm{mg}, 72 \%$ yield, $1.3: 1 \mathrm{dr})$.

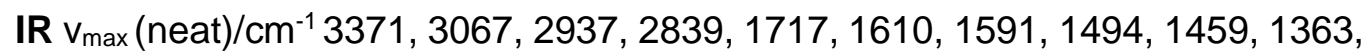
$1292,1250,1176,1120,1094,1025,968,935,884,828,782,736 .{ }^{1} \mathbf{H}$ NMR $(400$ $\left.\mathrm{MHz}, \mathrm{CD}_{2} \mathrm{Cl}_{2}, 25^{\circ} \mathrm{C}\right) \delta 7.39-7.34(\mathrm{~m}, 1 \mathrm{H}, \mathrm{ArH}), 7.22-7.14(\mathrm{~m}, 2 \mathrm{H}, \mathrm{ArH}), 7.07$ (dd, $J=8.3,0.8 \mathrm{~Hz}, 1 \mathrm{H}, \mathrm{ArH}), 6.98-6.89(\mathrm{~m}, 2 \mathrm{H}, \mathrm{ArH}), 6.77$ (dd, $J=7.9,0.9 \mathrm{~Hz}$, $1 \mathrm{H}, \mathrm{ArH}), 5.69(\mathrm{~d}, J=46.8 \mathrm{~Hz}, 1 \mathrm{H}, \mathrm{C}(\mathrm{F}) \mathrm{H}), 4.08(\mathrm{t}, J=2.1 \mathrm{~Hz}, 1 \mathrm{H}, \mathrm{OH}), 3.81(\mathrm{~s}$, $\left.3 \mathrm{H}, \mathrm{OCH}_{3}\right), 3.16\left(\mathrm{~s}, 3 \mathrm{H}, \mathrm{NCH}_{3}\right) .{ }^{13} \mathrm{C}$ NMR $\left(101 \mathrm{MHz}, \mathrm{CD}_{2} \mathrm{Cl}_{2}, 25^{\circ} \mathrm{C}\right) \delta 195.7$ (d, ${ }^{2} \mathrm{~J} \mathrm{C}-\mathrm{F}=27.6$ $\mathrm{Hz}$ ), 172.9, 161.6, 146.6, 136.6, 132.7, 132.4, 128.7, 124.6, 122.3 (d, ${ }^{3} \mathrm{~J} \mathrm{C}-\mathrm{F}=2.9 \mathrm{~Hz}$ ), 115.8, $115.8\left(\mathrm{~d},{ }^{3} \mathrm{~J} \mathrm{C}-\mathrm{F}=5.1 \mathrm{~Hz}\right), 107.8,94.3\left(\mathrm{~d},{ }^{1} \mathrm{~J} \mathrm{C}-\mathrm{F}=200.6 \mathrm{~Hz}\right), 77.1\left(\mathrm{~d},{ }^{2} J \mathrm{C}-\mathrm{F}=21.6 \mathrm{~Hz}\right), 55.8$, 26.9. ${ }^{19} \mathrm{~F}$ NMR $\left(377 \mathrm{MHz}, \mathrm{CD}_{2} \mathrm{Cl}_{2}, 25^{\circ} \mathrm{C}\right) \delta-199.50$ (d, $\left.{ }^{2} \mathrm{~J}_{\mathrm{HF}}=46.8 \mathrm{~Hz}\right)$. HRMS (ESI+): $\mathrm{m} / \mathrm{z}$ $[\mathrm{M}+\mathrm{Na}]^{+}$Calcd. for $\mathrm{C}_{18} \mathrm{H}_{15} \mathrm{CIFNO}_{4} \mathrm{SNa}$ : 418.0287; Found: 418.0284 . 


\section{S-(4-methoxyphenyl) (R)-2-((R)-4-chloro-3-hydroxy-2-oxoindolin-3-yl)-2- \\ fluoroethanethioate $(4 \mathrm{k})$}<smiles>COc1ccc(SC(=O)C(F)[C@]2(O)C(=O)Nc3cccc(Cl)c32)cc1</smiles>

4k

Prepared according to general procedure 2 using catalyst $F$. The product was purified by column chromatography (ethyl acetate / $n$-hexane) to afford the desired product as a white solid (27.3 $\mathrm{mg}, 70 \%$ yield, $1.5: 1 \mathrm{dr}$ ).

IR $\mathrm{v}_{\max }$ (neat)/ $/ \mathrm{cm}^{-1} 3369,2927,2849,2360,2338,1719,1606,1591,1494,1456$, 1361, 1292, 1251, 1176, 1109, 1026, 828, 778. 'H NMR $(600 \mathrm{MHz}$, d6-Acetone, $\left.25^{\circ} \mathrm{C}\right) \delta 9.65(\mathrm{~s}, 1 \mathrm{H}, \mathrm{NH}), 7.32-7.27(\mathrm{~m}, 1 \mathrm{H}, \mathrm{ArH}), 7.22-7.16(\mathrm{~m}, 2 \mathrm{H}, \mathrm{ArH})$, 7.01 (dd, $J=8.2,0.8 \mathrm{~Hz}, 1 \mathrm{H}, \mathrm{ArH}), 6.99-6.94(\mathrm{~m}, 2 \mathrm{H}, \mathrm{ArH}), 6.86$ (dd, $J=7.9$, $0.8 \mathrm{~Hz}, 1 \mathrm{H}, \mathrm{ArH}), 5.94(\mathrm{~s}, 1 \mathrm{H}, \mathrm{OH}), 5.78(\mathrm{~d}, J=47.0 \mathrm{~Hz}, 1 \mathrm{H}, \mathrm{C}(\mathrm{F}) \mathrm{H}), 3.81(\mathrm{~s}, 3 \mathrm{H}$, $\left.\mathrm{OCH}_{3}\right)$. Visible peaks of minor diastereoisomers: $9.73(\mathrm{~s}, 1 \mathrm{H}, \mathrm{NH}), 7.38-7.33(\mathrm{~m}, 1 \mathrm{H}, \mathrm{ArH})$, $7.19-7.14(\mathrm{~m}, 2 \mathrm{H}, \mathrm{ArH}), 7.08(\mathrm{dd}, J=8.2,0.9 \mathrm{~Hz}, 1 \mathrm{H}, \mathrm{ArH}), 7.01-6.95(\mathrm{~m}, 2 \mathrm{H}, \mathrm{ArH}), 6.91$ (dd, $J=7.8,0.9 \mathrm{~Hz}, 1 \mathrm{H}, \mathrm{ArH}), 5.65(\mathrm{~d}, J=47.5 \mathrm{~Hz}, 1 \mathrm{H}, \mathrm{C}(\mathrm{F}) \mathrm{H}), 5.57(\mathrm{~s}, 1 \mathrm{H}, \mathrm{OH}), 3.83(\mathrm{~s}, 3 \mathrm{H}$, $\left.\mathrm{OCH}_{3}\right) .{ }^{13} \mathrm{C}$ NMR $\left(151 \mathrm{MHz}\right.$, d6-Acetone, $\left.{ }^{\circ} 5^{\circ} \mathrm{C}\right) \delta 194.1$ (d, $\left.{ }^{2} \mathrm{~J} \mathrm{C}-\mathrm{F}=27.6 \mathrm{~Hz}\right), 132.9,132.8$, 124.3, 124.3, 124.1, 117.0 (d, ${ }^{3} J \mathrm{C}-\mathrm{F}=5.0 \mathrm{~Hz}$ ), 115.8, 109.7, 95.4 (d, $\left.{ }^{1} J \mathrm{C}-\mathrm{F}=200.6 \mathrm{~Hz}\right), 77.5$ $\left(\mathrm{d},{ }^{2} \mathrm{~J} \mathrm{C}-\mathrm{F}=21.5 \mathrm{~Hz}\right.$ ), 55.8. Visible peaks of minor diastereoisomers: 196.6 (d, ${ }^{2} \mathrm{~J} \mathrm{C}-\mathrm{F}=29.1$ $\mathrm{Hz}), 174.5\left(\mathrm{~d},{ }^{3} \mathrm{~J} \mathrm{C}-\mathrm{F}=3.9 \mathrm{~Hz}\right), 162.1,145.8,137.3,133.0,124.7,124.3,117.0\left(\mathrm{~d},{ }^{3} \mathrm{~J} \mathrm{C}-\mathrm{F}=\right.$ 6.3 Hz), 115.8, 109.9, 95.1 (d, $\left.{ }^{1} J \mathrm{C}-\mathrm{F}=198.4 \mathrm{~Hz}\right), 78.3\left(\mathrm{~d},{ }^{2} \mathrm{~J} \mathrm{C}-\mathrm{F}=22.8 \mathrm{~Hz}\right), 55.8 .{ }^{19} \mathrm{~F}$ NMR $\left(470 \mathrm{MHz}\right.$, d6-Acetone, $\left.25^{\circ} \mathrm{C}\right) \delta-200.20\left(\mathrm{~d},{ }^{2} J_{\mathrm{HF}}=47.1 \mathrm{~Hz}\right)$. Visible peaks of minor diastereoisomers: $-195.46\left(\mathrm{~d},{ }^{2} \mathrm{JHF}_{\mathrm{HF}}=47.4 \mathrm{~Hz}\right)$. HRMS (ESI+): $\mathrm{m} / \mathrm{z}[\mathrm{M}+\mathrm{Na}]^{+}$Calcd. for $\mathrm{C}_{17} \mathrm{H}_{13} \mathrm{CIFNO}_{4} \mathrm{SNa}$ : 404.0130; Found: 404.0128.

\section{S-(4-methoxyphenyl) (R)-2-((R)-4-bromo-3-hydroxy-1-methyl-2-oxoindolin-3-yl)-2-}

\section{fluoroethanethioate (4I)}

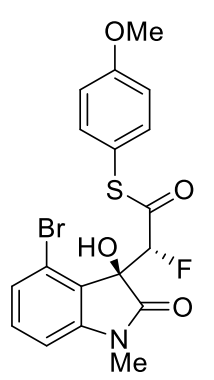

4!

Prepared according to general procedure 2 using catalyst $F$. The product was purified by column chromatography (ethyl acetate / $n$-hexane) to afford the desired product as a white solid (35.6 mg, 79\% yield, 1.5:1 dr).

IR $\mathrm{V}_{\max }\left(\right.$ neat) $/ \mathrm{cm}^{-1} 3341,3173,3098,2921,2851,2359,2337,1716,1694,1620$, 1591, 1493, 1452, 1296, 1251, 1178, 1098, 1027, 955, 917, 825, 790. 'H NMR $\left(500 \mathrm{MHz}, \mathrm{CD}_{2} \mathrm{Cl}_{2}, 25^{\circ} \mathrm{C}\right) \delta 7.29-7.22(\mathrm{~m}, 2 \mathrm{H}, \mathrm{ArH}), 7.18-7.11(\mathrm{~m}, 2 \mathrm{H}, \mathrm{ArH})$, $6.95-6.88(\mathrm{~m}, 2 \mathrm{H}, \mathrm{ArH}), 6.79(\mathrm{dd}, J=7.2,1.5 \mathrm{~Hz}, 1 \mathrm{H}, \mathrm{ArH}), 5.89-5.75(\mathrm{~m}, 1 \mathrm{H}$, $\mathrm{C}(\mathrm{F}) \mathrm{H}), 3.99(\mathrm{bs}, 1 \mathrm{H}, \mathrm{OH}), 3.80\left(\mathrm{~s}, 3 \mathrm{H}, \mathrm{OCH}_{3}\right), 3.14\left(\mathrm{~s}, 3 \mathrm{H}, \mathrm{NCH}_{3}\right) .{ }^{13} \mathrm{C}$ NMR (126 $\left.\mathrm{MHz}, \mathrm{CD}_{2} \mathrm{Cl}_{2}, 2^{\circ} \mathrm{C}\right) \delta 199.8\left(\mathrm{~d},{ }^{2} \mathrm{~J} \mathrm{C}-\mathrm{F}=28.7 \mathrm{~Hz}\right) 172.9,161.6,146.7,136.5,132.7,127.7$, $124.0\left(\mathrm{~d},{ }^{3} \mathrm{~J} \mathrm{C}-\mathrm{F}=3.6 \mathrm{~Hz}\right), 120.3,115.4,116.2\left(\mathrm{~d},{ }^{3} \mathrm{~J} \mathrm{C}-\mathrm{F}=6.5 \mathrm{~Hz}\right) 108.2,91.2\left(\mathrm{~d},{ }^{1} \mathrm{~J} \mathrm{C}-\mathrm{F}=\right.$ 196.3 Hz), 78.5 (d, $\left.{ }^{2} \mathrm{~J} \mathrm{C}-\mathrm{F}=24.6 \mathrm{~Hz}\right) 55.8,26.7 .{ }^{19} \mathrm{~F} \mathrm{NMR}\left(470 \mathrm{MHz}, \mathrm{CD}_{2} \mathrm{Cl}_{2}, 25^{\circ} \mathrm{C}\right) \delta-200.71$ $\left(\mathrm{d},{ }^{2} J_{\mathrm{HF}}=47.1 \mathrm{~Hz}\right)$. HRMS (ESI+): $\mathrm{m} / \mathrm{z}[\mathrm{M}+\mathrm{Na}]^{+}$Calcd. for $\mathrm{C}_{18} \mathrm{H}_{15} \mathrm{BrFNO}_{4} \mathrm{SNa}: 461.9781$; Found: 461.9783. 


\section{S-(4-methoxyphenyl) (R)-2-fluoro-2-((R)-3-hydroxy-1-methyl-2-oxo-4-phenylindolin-3-}

\section{yl)ethanethioate $(4 \mathrm{~m})$}

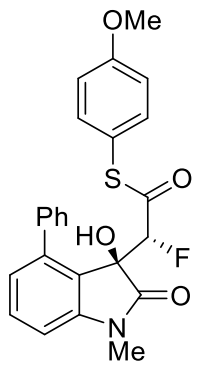

$4 \mathrm{~m}$

Prepared according to general procedure 2 using catalyst $F$. The product was purified by column chromatography (ethyl acetate / $n$-hexane) to afford the desired product as a orange solid (15.6 mg, 35\% yield, $1.4: 1 \mathrm{dr}$ ).

M.P. $170-174^{\circ} \mathrm{C}$. IR $v_{\max }$ (neat) $/ \mathrm{cm}^{-1} 3060,2928,1733 ; 1590,1461,1358,1311$, 1203, 1087. ${ }^{1} \mathrm{H}$ NMR $\left(600 \mathrm{MHz}, \mathrm{C}_{6} \mathrm{D}_{6}\right) \delta 7.80-7.74(\mathrm{~m}, 1 \mathrm{H}, \mathrm{ArH}), 7.29-7.23$ $(\mathrm{m}, 2 \mathrm{H}, \mathrm{ArH}), 6.98\left(\mathrm{t}, J=7.8 \mathrm{~Hz}, 1 \mathrm{H}, \mathrm{ArH}, 2^{\circ} \mathrm{C}\right), 6.94-6.87(\mathrm{~m}, 2 \mathrm{H}, \mathrm{ArH}), 6.83$ (t, $J=7.8 \mathrm{~Hz}, 1 \mathrm{H}, \mathrm{ArH}), 6.64(\mathrm{dd}, J=7.8,0.9 \mathrm{~Hz}, 1 \mathrm{H}, \mathrm{ArH}), 6.52-6.47(\mathrm{~m}, 2 \mathrm{H}$, ArH), $5.78(\mathrm{dd}, J=7.9,0.8 \mathrm{~Hz}, 1 \mathrm{H}, \mathrm{ArH}), 5.05(\mathrm{~d}, J=47.8 \mathrm{~Hz}, 1 \mathrm{H} \mathrm{C}(\mathrm{F}) \mathrm{H}), 3.07$ (s, 3H, OCH 3 ), 2.39 (s, 3H, NCH 3 ). ${ }^{13} \mathrm{C} \mathrm{NMR}\left(151 \mathrm{MHz}, \mathrm{C}_{6} \mathrm{D}_{6}, 25^{\circ} \mathrm{C}\right) \delta 193.3$ (d, ${ }^{2} \mathrm{~J} \mathrm{C}-\mathrm{F}=27.9$ $\mathrm{Hz}), 181.8,173.3,161.2,160.2,157.5,152.3,145.4,142.7$ (d, $\left.{ }^{3} \mathrm{~J} \mathrm{C}-\mathrm{F}=10.7 \mathrm{~Hz}\right), 139.3,136.5$ (d, $\left.{ }^{3} J \mathrm{C}-\mathrm{F}=18.6 \mathrm{~Hz}\right), 130.8,130.6,129.4,129.2,125.6,125.0,115.1,107.9,107.6,94.8$ (d, $\left.{ }^{1} \mathrm{~J} \mathrm{C}-\mathrm{F}=201.9 \mathrm{~Hz}\right), 76.5\left(\mathrm{~d},{ }^{2} \mathrm{~J} \mathrm{C}-\mathrm{F}=19.5 \mathrm{~Hz}\right), 54.7,30.2 .{ }^{19} \mathrm{~F} \mathrm{NMR}\left(470 \mathrm{MHz}, \mathrm{C}_{6} \mathrm{D}_{6}, 25^{\circ} \mathrm{C}\right) \delta$ $-199.17\left(\mathrm{~d},{ }^{2} \mathrm{JHF}_{\mathrm{HF}} 47.9 \mathrm{~Hz}\right)$. HRMS (ESI+): $\mathrm{m} / \mathrm{z}[\mathrm{M}+\mathrm{Na}]^{+}$Calcd. for $\mathrm{C}_{24} \mathrm{H}_{20} \mathrm{FNO}_{4} \mathrm{SNa}: 460.0989$; Found: 460.0989.

\section{S-(4-methoxyphenyl) (R)-2-((R)-4,7-dichloro-3-hydroxy-1-methyl-2-oxoindolin-3-yl)-2- fluoroethanethioate $(4 \mathrm{n})$}

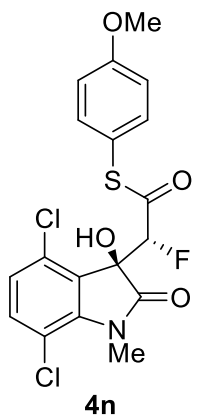

Prepared according to general procedure 2 using catalyst $F$. The product was purified by column chromatography (ethyl acetate / $n$-hexane) to afford the desired product as a white solid (34 $\mathrm{mg}, 97 \%$ yield, 1.4:1 dr).

IR $\mathrm{V}_{\max }$ (neat) $/ \mathrm{cm}^{-1} 3401,2953,2839.0,1733,1692,1594,1494,1450,1333$, 1292, 1251, 1180, 1112, 1026. ${ }^{1} \mathrm{H}$ NMR $\left(400 \mathrm{MHz}, \mathrm{C}_{6} \mathrm{D}_{6}, 25^{\circ} \mathrm{C}\right) \delta=7.06(\mathrm{~d}$, $\mathrm{J}=8.8,2 \mathrm{H}, \mathrm{ArH}), 6.60-6.51(\mathrm{~m}, 3 \mathrm{H}, \mathrm{ArH}), 6.44-6.36(\mathrm{~m}, 1 \mathrm{H}, \mathrm{ArH}), 5.75(\mathrm{~d}$, $\left.{ }^{2} J_{\mathrm{HF}}=46.8,1 \mathrm{H}, \mathrm{C}(\mathrm{F}) \mathrm{H}\right), 4.23(\mathrm{~s}, 1 \mathrm{H}, \mathrm{OH}), 3.11\left(\mathrm{~s}, 3 \mathrm{H}, \mathrm{OCH}_{3}\right), 3.01\left(\mathrm{~s}, 3 \mathrm{H}, \mathrm{NCH}_{3}\right)$. Visible peaks of minor diastereoisomer: $7.46(\mathrm{~d}, \mathrm{~J}=8.9,2 \mathrm{H}, \mathrm{ArH}), 6.69(\mathrm{~d}, \mathrm{~J}=8.9$, $2 \mathrm{H}, \mathrm{ArH}), 5.74\left(\mathrm{~d},{ }^{2} \mathrm{~J}_{\mathrm{HF}}=46.6,1 \mathrm{H}, \mathrm{C}(\mathrm{F}) \mathrm{H}\right), 4.90(\mathrm{~s}, 1 \mathrm{H}, \mathrm{OH}), 3.16\left(\mathrm{~s}, 3 \mathrm{H}, \mathrm{OCH}_{3}\right), 2.91(\mathrm{~s}, 3 \mathrm{H}$, $\left.\mathrm{NCH}_{3}\right) .{ }^{13} \mathrm{C}$ NMR $\left(101 \mathrm{MHz}, \mathrm{C}_{6} \mathrm{D}_{6}, 25^{\circ} \mathrm{C}\right) \delta=199.5\left(\mathrm{~d},{ }^{2} \mathrm{~J} \mathrm{C}-\mathrm{F}=28.6\right), 173.2,161.4,142.3$, 136.5, 133.9, 131.1, 128.6, 124.8, $116.0\left(\mathrm{t},{ }^{3} J \mathrm{C}-\mathrm{F}=5.9\right), 115.4,114.6,94.2\left(\mathrm{~d},{ }^{1} J \mathrm{C}-\mathrm{F}=202.0\right)$, $76.7\left(\mathrm{~d},{ }^{2} \mathrm{~J} \mathrm{C}-\mathrm{F}=21.4\right), 54.8,29.3$. Visible peaks of minor diastereoisomer: $194.9\left(\mathrm{~d},{ }^{2} \mathrm{~J} \mathrm{C}-\mathrm{F}=\right.$ 28.0), 173.3, 161.6, 142.7, 136.9, 134.1, 125.0, 116.0 (t, $\left.{ }^{3} J \mathrm{C}-\mathrm{F}=5.9\right), 115.5,114.8,91.2$ (d, $\left.{ }^{1} \mathrm{~J} \mathrm{C}-\mathrm{F}=197.0\right), 77.6\left(\mathrm{~d},{ }^{2} \mathrm{~J} \mathrm{C}-\mathrm{F}=24.4\right), 54.8,29.4 .{ }^{19} \mathrm{~F}$ NMR $\left(377 \mathrm{MHz}, \mathrm{C}_{6} \mathrm{D}_{6}, 25^{\circ} \mathrm{C}\right) \delta=-$ $198.78\left(\mathrm{~d},{ }^{2} J_{\mathrm{HF}}=46.6\right)$. Visible peaks of minor diastereoisomer: $-195.02\left(\mathrm{~d},{ }^{2} J_{\mathrm{HF}}=46.6\right)$. HRMS (ESI+): $\mathrm{m} / \mathrm{z}[\mathrm{M}+\mathrm{H}]^{+}$Calcd. for $\mathrm{C}_{18} \mathrm{H}_{15} \mathrm{Cl}_{2} \mathrm{FNO}_{4} \mathrm{~S}: 430.0077$; Found: 430.0075 . 


\section{S-(4-methoxyphenyl) (R)-2-((R)-4,7-dichloro-3-hydroxy-2-oxoindolin-3-yl)-2- fluoroethanethioate(40)}

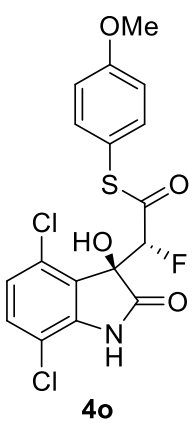

Prepared according to general procedure 2 using catalyst $F$. The product was purified by column chromatography (ethyl acetate / $n$-hexane) to afford the desired product as a white solid (36.0 mg, 94\% yield, 2.0:1 dr).

IR $\mathbf{v}_{\max }$ (neat) $/ \mathrm{cm}^{-1} 3271,2941,2837,2360,1736,1689,1614,1591,1493,1468$, 1432, 1292, 1249, 1162, 1107, 1094, 1026, 952, 928, 826, 798, 708. 'H NMR $\left(400 \mathrm{MHz}, \mathrm{CD}_{2} \mathrm{Cl}_{2}, 25^{\circ} \mathrm{C}\right) \delta 7.28(\mathrm{~d}, J=8.7 \mathrm{~Hz}, 1 \mathrm{H}, \mathrm{ArH}), 7.26-7.18(\mathrm{~m}, 2 \mathrm{H}$, $\operatorname{ArH}), 7.03(\mathrm{~d}, J=8.8 \mathrm{~Hz}, 1 \mathrm{H}, \mathrm{ArH}), 6.98-6.89(\mathrm{~m}, 2 \mathrm{H}, \mathrm{ArH}), 5.71(\mathrm{~d}, J=46.6$ $\mathrm{Hz}, 1 \mathrm{H}, \mathrm{C}(\mathrm{F}) \mathrm{H}), 3.80\left(\mathrm{~s}, 3 \mathrm{H}, \mathrm{OCH}_{3}\right)$. Visible peaks of minor diastereoisomer: $\delta$ $7.37-7.33(\mathrm{~m}, 2 \mathrm{H}, \mathrm{ArH}), 7.32(\mathrm{~d}, J=8.8 \mathrm{~Hz}, 1 \mathrm{H}, \mathrm{ArH}), 7.05(\mathrm{~d}, J=8.8 \mathrm{~Hz}, 1 \mathrm{H}, \mathrm{ArH}), 7.02-$ $6.97(\mathrm{~m}, 2 \mathrm{H}, \mathrm{ArH}), 5.73(\mathrm{~d}, J=46.4 \mathrm{~Hz}, 1 \mathrm{H}, \mathrm{C}(\mathrm{F}) \mathrm{H}), 3.84\left(\mathrm{~s}, 3 \mathrm{H}, \mathrm{OCH}_{3}\right) .{ }^{13} \mathrm{C}$ NMR $(101 \mathrm{MHz}$, $\left.\mathrm{CD}_{2} \mathrm{Cl}_{2}, 2^{\circ} \mathrm{C}\right) \delta 195.8\left(\mathrm{~d},{ }^{2} \mathrm{~J} \mathrm{C}-\mathrm{F}=27.7 \mathrm{~Hz}\right), 173.9,161.8,161.7,141.1,136.8,136.6,132.5$, 132.4, 131.0, 125.6, 125.5, 123.9 (d, ${ }^{3} J \mathrm{C}-\mathrm{F}=3.0 \mathrm{~Hz}$ ), 115.6, 115.5, 115.5, 115.0, 114.9, 94.0 $\left(\mathrm{d},{ }^{1} \mathrm{~J} \mathrm{C}-\mathrm{F}=201.1 \mathrm{~Hz}\right), 78.3\left(\mathrm{~d},{ }^{2} \mathrm{~J} \mathrm{C}-\mathrm{F}=21.6 \mathrm{~Hz}\right), 55.8$. Visible peaks of minor diastereoisomer: $\delta 199.7\left(\mathrm{~d},{ }^{2} \mathrm{~J} \mathrm{C}-\mathrm{F}=28.6 \mathrm{~Hz}\right), 173.6,161.8,141.6,136.8,132.5,132.4,125.5,115.6,91.2(\mathrm{~d}$, $\left.{ }^{1} \mathrm{~J} \mathrm{C}-\mathrm{F}=196.9 \mathrm{~Hz}\right), 79.0\left(\mathrm{~d},{ }^{2} \mathrm{~J} \mathrm{C}-\mathrm{F}=24.6 \mathrm{~Hz}\right), 55.8,30.1 .{ }^{19} \mathrm{~F} \mathrm{NMR}\left(376 \mathrm{MHz}, \mathrm{CD}_{2} \mathrm{Cl}_{2}, 25^{\circ} \mathrm{C}\right)$ $\delta-199.58\left(\mathrm{~d},{ }^{2} \mathrm{JHF}_{\mathrm{HF}}=46.5 \mathrm{~Hz}\right)$. Visible peaks of minor diastereoisomer: $\delta-195.55\left(\mathrm{~d},{ }^{2} \mathrm{JHF}_{\mathrm{HF}}=46.3\right.$ $\mathrm{Hz}$ ). HRMS (ESI+): $\mathrm{m} / \mathrm{z}[\mathrm{M}+\mathrm{Na}]^{+}$Calcd. for $\mathrm{C}_{17} \mathrm{H}_{12} \mathrm{Cl}_{2} \mathrm{FNO}_{4} \mathrm{SNa}: 437.9740$; Found: 437.9741.

\section{S-(4-methoxyphenyl) (R)-2-((R)-1-benzyl-4,7-dichloro-3-hydroxy-2-oxoindolin-3-yl)-2- fluoroethanethioate $(4 p)$}<smiles>COc1ccc(SC(=O)C(F)[C@]2(O)C(=O)C(=O)c3c(Cl)ccc(Cl)c32)cc1</smiles>

$\left(\mathrm{m}, 2 \mathrm{H}, \mathrm{ArCH}_{2}\right), 3.14\left(\mathrm{~s}, 3 \mathrm{H}, \mathrm{OCH}_{3}\right)$. Visible peaks of minor diastereoisomer $6.61-6.56(\mathrm{~m}$, $2 \mathrm{H}, \mathrm{ArH}), 6.47(\mathrm{~d}, J=8.7 \mathrm{~Hz}, 1 \mathrm{H}), \operatorname{ArH}, 6.36(\mathrm{~d}, \mathrm{~J}=8.8 \mathrm{~Hz}, 1 \mathrm{H}, \operatorname{ArH}), 5.90(\mathrm{~d}, \mathrm{~J}=46.5 \mathrm{~Hz}$, $1 \mathrm{H}, \mathrm{C}(\mathrm{F}) \mathrm{H}), 5.09(\mathrm{~d}, J=16.4 \mathrm{~Hz}, 1 \mathrm{H}, \mathrm{ArCH}), 4.96(\mathrm{~d}, \mathrm{~J}=16.5 \mathrm{~Hz}, 1 \mathrm{H}, \mathrm{ArCH}), 3.14(\mathrm{~s}, 3 \mathrm{H}$, $\left.\mathrm{OCH}_{3}\right) .{ }^{13} \mathrm{C}$ NMR $\left(101 \mathrm{MHz}, \mathrm{C}_{6} \mathrm{D}_{6}, 25^{\circ} \mathrm{C}\right) \delta 199.7$ (d, $\left.{ }^{2} \mathrm{~J} \mathrm{C}-\mathrm{F}=28.6 \mathrm{~Hz}\right), 174.1,161.7,142.1$, 137.1, 136.9, 134.3, 131.2, 129.0, 127.5, 126.4, 125.3, 125.0, 115.9 (d, $\left.{ }^{3} J \mathrm{C}-\mathrm{F}=6.4 \mathrm{~Hz}\right), 115.5$, 115.0, 114.8, 90.9 (d, $\left.{ }^{1} \mathrm{~J} \mathrm{C}-\mathrm{F}=196.3 \mathrm{~Hz}\right), 77.6\left(\mathrm{~d},{ }^{2} \mathrm{~J} \mathrm{C}-\mathrm{F}=24.8 \mathrm{~Hz}\right), 54.8,45.2$. Visible peaks of minor diastereoisomer 173.4, 161.9, 137.6, 136.6, 134.2, 129.0, 126.5, 125.1, 115.3, 93.9 $\left(\mathrm{d},{ }^{1} \mathrm{~J} \mathrm{C}-\mathrm{F}=201.2 \mathrm{~Hz}\right), 54.8,45.4 .{ }^{19} \mathrm{~F}$ NMR $\left(376 \mathrm{MHz}, \mathrm{C}_{6} \mathrm{D}_{6}, 25^{\circ} \mathrm{C}\right) \delta-194.53\left(\mathrm{~d},{ }^{2} \mathrm{JHF}_{\mathrm{HF}}=46.6\right.$ $\mathrm{Hz}$ ). Visible peaks of minor diastereoisomer: $\delta-198.97\left(\mathrm{~d},{ }^{2} \mathrm{~J}_{\mathrm{HF}}=46.5 \mathrm{~Hz}\right) . \mathbf{H R M S}(\mathbf{E S I}+): \mathrm{m} / \mathrm{z}$ $[\mathrm{M}+\mathrm{Na}]^{+}$Calcd. for $\mathrm{C}_{24} \mathrm{H}_{18} \mathrm{Cl}_{2} \mathrm{FNO}_{4} \mathrm{SNa}$ : 528.0210; Found: 528.0209. 


\section{S-(4-methoxyphenyl) (R)-2-fluoro-2-((R)-3-hydroxy-1-methyl-2-oxo-5-}

\section{(trifluoromethoxy)indolin-3-yl)ethanethioate (4q)}

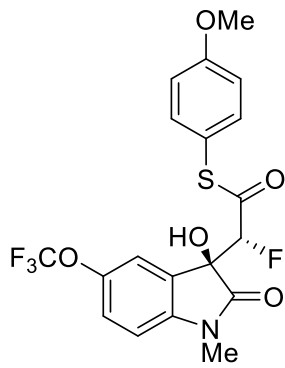

$4 q$

Prepared according to general procedure 2 using catalyst $F$. The product was purified by column chromatography (ethyl acetate / $n$-hexane) to afford the desired product as a white solid $(31.6 \mathrm{mg}, 87 \%$ yield, $1.8: 1 \mathrm{dr})$.

IR $\mathbf{v}_{\max }$ (neat)/ $/ \mathrm{cm}^{-1} 3371,2942,2845.1715 ; 1621,1592,1495,1368,1251$, 1214, 1172, 1109, 1029. ${ }^{1} \mathrm{H}$ NMR $\left(400 \mathrm{MHz}, \mathrm{C}_{6} \mathrm{D}_{6}, 25^{\circ} \mathrm{C}\right) \delta=7.47(\mathrm{~d}, \mathrm{~J}=2.4$, $1 \mathrm{H}, \mathrm{ArH}), 7.08$ (d, J=8.7, 2H, ArH), $6.87-6.75(\mathrm{~m}, 1 \mathrm{H}, \mathrm{ArH}), 6.57$ (d, J=8.8, $2 \mathrm{H}, \mathrm{ArH}), 5.81(\mathrm{~d}, J=8.5,1 \mathrm{H}, \mathrm{ArH}), 5.17\left(\mathrm{~d},{ }^{2} J_{\mathrm{HF}}=47.7,1 \mathrm{H}, \mathrm{C}(\mathrm{F}) \mathrm{H}\right), 4.50(\mathrm{~s}$, $1 \mathrm{H}, \mathrm{OH}), 3.10\left(\mathrm{~s}, 3 \mathrm{H}, \mathrm{OCH}_{3}\right), 2.42\left(\mathrm{~s}, 3 \mathrm{H}, \mathrm{NCH}_{3}\right)$. Visible peaks of minor diastereoisomer: $7.33-7.30(\mathrm{~m}, 1 \mathrm{H}, \mathrm{ArH}), 7.21(\mathrm{~d}, \mathrm{~J}=8.7,2 \mathrm{H}, \operatorname{ArH}), 6.68(\mathrm{~d}, \mathrm{~J}=8.7,2 \mathrm{H}, \mathrm{ArH})$, $5.32\left(\mathrm{~d},{ }^{2} J_{\mathrm{HF}}=45.8,1 \mathrm{H}, \mathrm{C}(\mathrm{F}) \mathrm{H}\right), 3.16\left(\mathrm{~s}, 3 \mathrm{H}, \mathrm{OCH}_{3}\right) .{ }^{13} \mathrm{C} \mathrm{NMR}\left(101 \mathrm{MHz}, \mathrm{C}_{6} \mathrm{D}_{6}\right) \delta=197.0\left(\mathrm{~d},{ }^{2} \mathrm{~J}\right.$ $\mathrm{C}-\mathrm{F}=28.9), 173.6\left(\mathrm{~d},{ }^{3} \mathrm{~J} \mathrm{C}-\mathrm{F}=4.9\right), 161.4,145.0\left(\mathrm{~d},{ }^{3} \mathrm{~J} \mathrm{C}-\mathrm{F}=2.1\right), 143.6,136.6,127.5,123.9$, $121.4\left(\mathrm{~d},{ }^{1} \mathrm{~J} \mathrm{CF}_{3}=256.6\right), 119.8,115.9\left(\mathrm{~d},{ }^{3} \mathrm{~J} \mathrm{C}-\mathrm{F}=6.4\right), 115.3,109.2,95.7\left(\mathrm{~d},{ }^{1} \mathrm{~J}=199.3\right)$, $76.6\left(\mathrm{~d},{ }^{2} \mathrm{~J} \mathrm{C}-\mathrm{F}=20.9\right), 54.8,25.9$. Visible peaks of minor diastereoisomer: $197.0\left(\mathrm{~d},{ }^{2} \mathrm{~J} \mathrm{C}-\mathrm{F}\right.$ $=27.9$ ), 161.6, 143.7, 136.6, 127.2, 119.7, 115.8 (d, $\left.{ }^{3} J \mathrm{C}-\mathrm{F}=5.5\right), 115.5,109.5,94.1$ (d, ${ }^{1} J \mathrm{C}-\mathrm{F}$ $=196.5), 76.2\left(\mathrm{~d},{ }^{2} \mathrm{~J} \mathrm{C}-\mathrm{F}=24.4\right), 54.9,25.9 .{ }^{19} \mathrm{~F}$ NMR $\left(376 \mathrm{MHz}, \mathrm{C}_{6} \mathrm{D}_{6}, 25^{\circ} \mathrm{C}\right) \delta=-58.20$, $200.52\left(\mathrm{~d},{ }^{2} J_{\mathrm{HF}}=47.7\right)$. Visible peaks of minor diastereoisomer: $-58.05,-193.83\left(\mathrm{~d},{ }^{2} J_{\mathrm{HF}}=45.8\right)$. HRMS (ESI+): $\mathrm{m} / \mathrm{z}[\mathrm{M}+\mathrm{H}]^{+}$Calcd. for $\mathrm{C}_{19} \mathrm{H}_{16} \mathrm{~F}_{4} \mathrm{NO}_{5} \mathrm{~S}$ : 446.0680; Found: 446.0674.

\section{S-(4-methoxyphenyl) (R)-2-((R)-6-chloro-3-hydroxy-1-methyl-2-oxoindolin-3-yl)-2- fluoroethanethioate (4r)}

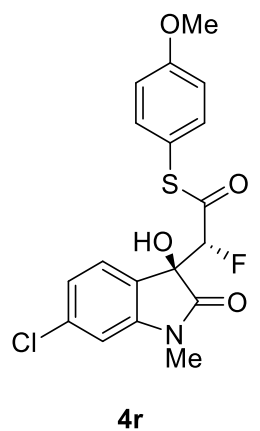

Prepared according to general procedure 2 using catalyst $F$. The product was purified by column chromatography (ethyl acetate / $n$-hexane) to afford the desired product as a white solid (30.7 mg, 95\% yield, 1.8:1 dr).

IR $\mathrm{V}_{\max }$ (neat)/cm ${ }^{-1} 3358,2923,2851,2355,1730,1699,1611,1592,1494$, 1463, 1436, 1374, 1291, 1250, 1174, 1107, 1073, 1027, 1002, 960, 872, 825, 741, 685. ${ }^{1} \mathrm{H}$ NMR $\left(400 \mathrm{MHz}, \mathrm{C}_{6} \mathrm{D}_{6}, 25^{\circ} \mathrm{C}\right) \delta=7.21(\mathrm{~d}, \mathrm{~J}=8.2,1 \mathrm{H}, \mathrm{ArH}), 7.09$ $(\mathrm{d}, \mathrm{J}=8.8,2 \mathrm{H}, \mathrm{ArH}), 6.80(\mathrm{td}, \mathrm{J}=8.2,1.9,1 \mathrm{H}, \mathrm{ArH}), 6.57(\mathrm{~d}, \mathrm{~J}=8.8,2 \mathrm{H}, \mathrm{ArH})$, $6.24(\mathrm{t}, \mathrm{J}=1.9,1 \mathrm{H}, \mathrm{ArH}), 5.19\left(\mathrm{~d},{ }^{2} \mathrm{~J}_{\mathrm{HF}}=47.7,1 \mathrm{H}, \mathrm{C}(\mathrm{F}) \mathrm{H}\right), 4.33(\mathrm{~s}, 1 \mathrm{H}, \mathrm{OH}), 3.10$ $\left(\mathrm{s}, 3 \mathrm{H}, \mathrm{OCH}_{3}\right), 2.33\left(\mathrm{~s}, 3 \mathrm{H}, \mathrm{NCH}_{3}\right)$. Visible peaks of minor diastereoisomer: $7.17(\mathrm{~d}, \mathrm{~J}=8.7,2 \mathrm{H}, \mathrm{ArH}), 6.68(\mathrm{~d}, \mathrm{~J}=8.8,2 \mathrm{H}, \mathrm{ArH}), 5.33\left(\mathrm{~d},{ }^{2} \mathrm{JHF}_{\mathrm{HF}}=46.0,1 \mathrm{H}, \mathrm{C}(\mathrm{F}) \mathrm{H}\right), 4.43(\mathrm{~s}, 1 \mathrm{H}$, $\mathrm{OH}), 3.18\left(\mathrm{~s}, 3 \mathrm{H}, \mathrm{OCH}_{3}\right), 2.32\left(\mathrm{~s}, 3 \mathrm{H}, \mathrm{NCH}_{3}\right) .{ }^{13} \mathrm{C} \mathrm{NMR}\left(101 \mathrm{MHz}, \mathrm{C}_{6} \mathrm{D}_{6}, 25^{\circ} \mathrm{C}\right) \delta=197.1(\mathrm{~d}$, $\left.{ }^{2} J \mathrm{C}-\mathrm{F}=29.0\right), 173.6\left(\mathrm{~d},{ }^{3} \mathrm{~J} \mathrm{C}-\mathrm{F}=14.5\right), 161.5,146.2,136.7,136.7,126.8,124.5,122.9,116.0$ $\left(\mathrm{d},{ }^{3} J \mathrm{C}-\mathrm{F}=6.4\right), 115.4,109.6,95.8\left(\mathrm{~d},{ }^{1} J \mathrm{C}-\mathrm{F}=199.0\right), 76.3\left(\mathrm{~d},{ }^{2} J \mathrm{C}-\mathrm{F}=20.9\right), 54.8,25.8$. Visible peaks of minor diastereoisomer: $196.7\left(\mathrm{~d},{ }^{2} \mathrm{~J} \mathrm{C}-\mathrm{F}=27.6\right), 161.6,146.4,136.8,136.6$, 126.6, 124.1, 122.8, 116.1 (d, $\left.{ }^{3} J \mathrm{C}-\mathrm{F}=5.3\right), 115.5,109.9,94.2\left(\mathrm{~d},{ }^{1} J_{\mathrm{C}-\mathrm{F}}=196.4\right), 75.9\left(\mathrm{~d},{ }^{2} J_{\mathrm{C}-\mathrm{F}}\right.$ $=24.4), 54.9,25.8 .{ }^{19} \mathrm{~F}$ NMR $\left(376 \mathrm{MHz}, \mathrm{C}_{6} \mathrm{D}_{6}, 25^{\circ} \mathrm{C}\right) \delta=-200.33\left(\mathrm{~d},{ }^{2} \mathrm{JHF}_{\mathrm{HF}}=47.4\right)$. Visible peaks of minor diastereoisomer: $-193.35\left(\mathrm{~d},{ }^{2} \mathrm{JHF}_{\mathrm{H}}=45.8\right)$. HRMS (ESI+): $\mathrm{m} / \mathrm{z}[\mathrm{M}+\mathrm{Na}]^{+}$Calcd. for $\mathrm{C}_{18} \mathrm{H}_{15} \mathrm{CIFNO}_{4} \mathrm{SNa}: 418.0287$; Found: 418.0288. 


\section{S-(4-methoxyphenyl) (R)-2-fluoro-2-((R)-3-hydroxy-1-methyl-2-oxo-7-}

(trifluoromethyl)indolin-3-yl)ethanethioate (4s)

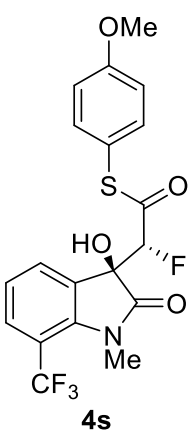
$5.19\left(\mathrm{~d},{ }^{2} J_{\mathrm{HF}}=46.1,1 \mathrm{H}, \mathrm{C}(\mathrm{F}) \mathrm{H}\right), 3.17\left(\mathrm{~s}, 3 \mathrm{H}, \mathrm{OCH}_{3}\right) .{ }^{13} \mathrm{C}$ NMR $\left(101 \mathrm{MHz}, \mathrm{C}_{6} \mathrm{D}_{6}, 25^{\circ} \mathrm{C}\right) \delta=197.0$ $\left(\mathrm{d},{ }^{2} J_{\mathrm{C}-\mathrm{F}}=29.0\right), 174.9-174.6(\mathrm{~m}), 161.5,142.9\left(\mathrm{~d},{ }^{4} J_{\mathrm{C}-\mathrm{F}}=1.9\right), 136.7,129.2,128.6\left(\mathrm{~d},{ }^{4} J_{\mathrm{C}-\mathrm{F}}\right.$ $=1.3$ ), 125.1, 122.6, 122.4, 122.4, $115.8\left(\mathrm{~d},{ }^{3} J_{\mathrm{C}-\mathrm{F}}=6.2\right), 115.4,112.9\left(\mathrm{~d},{ }^{2} J_{\mathrm{C}-\mathrm{F}}=33.2\right), 95.8(\mathrm{~d}$, $\left.{ }^{1} J_{\mathrm{C}-\mathrm{F}}=200.3\right), 54.8,28.9\left(\mathrm{q},{ }^{3} \mathrm{~J}_{\mathrm{C}-\mathrm{F}}=6.5\right)$. Visible peaks of minor diastereoisomer: $195.8\left(\mathrm{~d},{ }^{2} \mathrm{~J}_{\mathrm{C}-\mathrm{F}}\right.$ $=27.5), 161.6,136.5,129.0,116.0\left(\mathrm{~d},{ }^{3} J_{\mathrm{C}-\mathrm{F}}=5.2\right), 115.5,94.5\left(\mathrm{~d},{ }^{1} J_{\mathrm{C}-\mathrm{F}}=198.3\right), 74.6\left(\mathrm{~d},{ }^{2} J_{\mathrm{C}-\mathrm{F}}\right.$ $=23.5), 54.9 .{ }^{19} \mathrm{~F}$ NMR $\left(376 \mathrm{MHz}, \mathrm{C}_{6} \mathrm{D}_{6}\right) \delta=-53.39,-200.17\left(\mathrm{~d},{ }^{2} J_{\mathrm{HF}}=47.9\right)$. Visible peaks of minor diastereoisomer: $-53.34,-193.86\left(\mathrm{~d},{ }^{2} \mathrm{JHF}_{\mathrm{HF}}=46.0\right)$. HRMS (ESI+): $\mathrm{m} / \mathrm{z}[\mathrm{M}+\mathrm{H}]^{+}$Calcd. for $\mathrm{C}_{19} \mathrm{H}_{16} \mathrm{~F}_{4} \mathrm{NO}_{4} \mathrm{~S}$ : 430.0731; Found: 430.0732 .

\section{Methyl (R)-3-((R)-1-fluoro-2-((4-methoxyphenyl)thio)-2-oxoethyl)-3-hydroxy-1-methyl-2-} oxoindoline-7-carboxylate (4t)

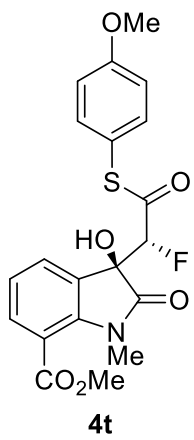

Prepared according to general procedure 2 using catalyst $F$. The product was purified by column chromatography (ethyl acetate / $n$-hexane) to afford the desired product as a white solid (38.0 mg, 98\% yield, 1.5:1 dr).

IR $\mathbf{v}_{\max }($ neat $) / \mathrm{cm}^{-1} 3391,2950,2839,1718,1594,1494,1458,1294,1249,1202$, 1111, 1028. ${ }^{1} \mathrm{H}$ NMR $\left(400 \mathrm{MHz}, \mathrm{C}_{6} \mathrm{D}_{6}, 25^{\circ} \mathrm{C}\right) \delta=7.52$ (ddd, J=7.3, 1.4, 0.9, $1 \mathrm{H}$, $\operatorname{ArH}), 7.47(\mathrm{~d}, J=8.0,1 \mathrm{H}, \operatorname{ArH}), 7.11(\mathrm{~d}, J=8.8,2 \mathrm{H}, \operatorname{ArH}), 6.75-6.65(\mathrm{~m}, 1 \mathrm{H}$, $\mathrm{ArH}), 6.60(\mathrm{~d}, J=8.9,2 \mathrm{H}, \mathrm{ArH}), 5.22\left(\mathrm{~d},{ }^{2} J_{\mathrm{HF}}=47.9,1 \mathrm{H}, \mathrm{C}(\mathrm{F}) \mathrm{H}\right), 4.40(\mathrm{~s}, 1 \mathrm{H}$, $\mathrm{OH}), 3.31\left(\mathrm{~s}, 3 \mathrm{H}, \mathrm{OCH}_{3}\right), 3.12\left(\mathrm{~s}, 3 \mathrm{H}, \mathrm{OCH}_{3}\right), 2.95\left(\mathrm{~s}, 3 \mathrm{H}, \mathrm{NCH}_{3}\right)$. Visible peaks of minor diastereoisomer: $7.36(\mathrm{dd}, J=7.4,1.3,1 \mathrm{H}, \mathrm{ArH}), 7.20(\mathrm{~d}, J=8.9,2 \mathrm{H}$, ArH), $5.25\left(\mathrm{~d},{ }^{2} J_{\mathrm{HF}}=46.2,1 \mathrm{H}, \mathrm{C}(\mathrm{F}) \mathrm{H}\right), 4.49(\mathrm{~s}, 1 \mathrm{H}, \mathrm{OH}), 3.34\left(\mathrm{~s}, 3 \mathrm{H}, \mathrm{OCH}_{3}\right), 3.18\left(\mathrm{~s}, 3 \mathrm{H}, \mathrm{OCH}_{3}\right)$, $2.95\left(\mathrm{~s}, 3 \mathrm{H}, \mathrm{NCH}_{3}\right) .{ }^{13} \mathrm{C}$ NMR $\left(101 \mathrm{MHz}, \mathrm{C}_{6} \mathrm{D}_{6}, 25^{\circ} \mathrm{C}\right) \delta=196.9\left(\mathrm{~d},{ }^{2} \mathrm{~J}_{\mathrm{C}-\mathrm{F}}=29.0\right), 175.2,175.1$ $\left(\mathrm{d},{ }^{3} J_{\mathrm{C}-\mathrm{F}}=4.6\right), 166.4,161.4,144.0,136.8,132.0,128.6,122.5,116.7,116.1$ (d, $\left.{ }^{3} J_{\mathrm{C}-\mathrm{F}}=6.2\right)$, 115.4, $96.0\left(\mathrm{~d},{ }^{1} \mathrm{~J}_{\mathrm{C}-\mathrm{F}}=200.0\right), 75.6\left(\mathrm{~d},{ }^{2} \mathrm{~J}_{\mathrm{C}-\mathrm{F}}=20.8\right), 54.8,51.8,30.2$. Visible peaks of minor diastereoisomer: $195.7\left(\mathrm{~d},{ }^{2} \mathrm{~J}_{\mathrm{C}-\mathrm{F}}=27.6\right), 166.3,161.5,144.2,136.6,132.2,122.3,116.8,116.3$ $\left(\mathrm{d},{ }^{3} J_{\mathrm{C}-\mathrm{F}}=5.0\right), 115.4,94.7\left(\mathrm{~d},{ }^{1} J_{\mathrm{C}-\mathrm{F}}=198.0\right), 75.2\left(\mathrm{~d},{ }^{2} J_{\mathrm{C}-\mathrm{F}}=23.6\right), 54.9,51.8,30.3 .{ }^{19} \mathrm{~F}$ NMR $\left(377 \mathrm{MHz}, \mathrm{C}_{6} \mathrm{D}_{6}, 25^{\circ} \mathrm{C}\right) \delta=-199.99\left(\mathrm{~d},{ }^{2} \mathrm{~J}_{\mathrm{HF}}=47.8\right)$. Visible peaks of minor diastereoisomer: $193.50\left(\mathrm{~d},{ }^{2} \mathrm{JHF}_{\mathrm{HF}}=46.3\right)$. HRMS (ESI+): $\mathrm{m} / \mathrm{z}[\mathrm{M}+\mathrm{H}]^{+}$Calcd. for $\mathrm{C}_{20} \mathrm{H}_{19} \mathrm{FNO}_{6} \mathrm{~S}: 420.0912$; Found: 420.0908 . 


\section{S-(4-methoxyphenyl) (R)-2-fluoro-2-((R)-3-hydroxy-1,5,7-trimethyl-2-oxoindolin-3-}

yl)ethanethioate $(4 \mathrm{u})$

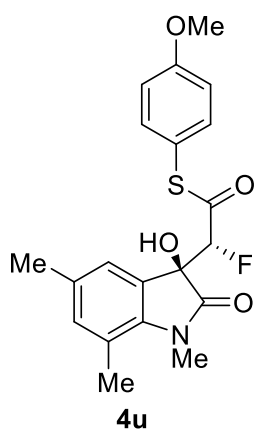

Prepared according to general procedure 2 using catalyst $F$. The product was purified by column chromatography (ethyl acetate / $n$-hexane) to afford the desired product as a white solid $(28.0 \mathrm{mg}, 87 \%$ yield, $1.8: 1 \mathrm{dr})$.

IR $\mathbf{V}_{\max }$ (neat)/cm ${ }^{-1} 3368,2937,1698,1592,1492,1460,1359,1290,1175$, 1094, 1028. ${ }^{1} \mathrm{H}$ NMR $\left(500 \mathrm{MHz}, \mathrm{C}_{6} \mathrm{D}_{6}, 25^{\circ} \mathrm{C}\right) \delta=7.26(\mathrm{~d}, J=8.9,2 \mathrm{H}, \mathrm{ArH})$, $6.61(\mathrm{~d}, J=8.9,2 \mathrm{H}, \mathrm{ArH}), 6.60-6.55(\mathrm{~m}, 1 \mathrm{H}, \mathrm{ArH}), 5.15\left(\mathrm{~d},{ }^{2} J_{\mathrm{HF}}=47.5,1 \mathrm{H}\right.$, $\mathrm{C}(\mathrm{F}) \mathrm{H}), 4.36(\mathrm{~s}, 1 \mathrm{H}, \mathrm{OH}), 3.12\left(\mathrm{~s}, 3 \mathrm{H}, \mathrm{OCH}_{3}\right), 2.80\left(\mathrm{~s}, 3 \mathrm{H}, \mathrm{NCH}_{3}\right), 2.07$ (s, $\left.3 \mathrm{H}, \mathrm{ArCH}_{3}\right), 1.85\left(\mathrm{~s}, 3 \mathrm{H}, \mathrm{ArCH}_{3}\right)$. Visible peaks of minor diastereoisomer: 7.21 (d, $J=8.9,2 \mathrm{H}, \mathrm{ArH}), 6.67(\mathrm{~d}, J=8.9,2 \mathrm{H}, \mathrm{ArH}), 5.38\left(\mathrm{dd},{ }^{2} J_{\mathrm{HF}}=46.2,3.5,1 \mathrm{H}\right.$, $\mathrm{C}(\mathrm{F}) \mathrm{H}), 4.25(\mathrm{~s}, 1 \mathrm{H}, \mathrm{OH}), 3.17\left(\mathrm{~s}, 3 \mathrm{H}, \mathrm{OCH}_{3}\right), 2.81$ (s, 3H, $\left.\mathrm{NCH}_{3}\right), 2.06$ (s, 3H, $\left.\mathrm{ArCH}_{3}\right), 1.86$ (d, J=0.8, 3H, $\left.\mathrm{ArCH}_{3}\right) .{ }^{13} \mathrm{C}$ NMR $\left(126 \mathrm{MHz}, \mathrm{C}_{6} \mathrm{D}_{6}, 25^{\circ} \mathrm{C}\right) \delta=197.9\left(\mathrm{~d},{ }^{2} \mathrm{~J}_{\mathrm{C}-\mathrm{F}}=29.4\right), 174.5(\mathrm{~d}$, $\left.{ }^{3} J_{C-F}=3.3\right), 161.4,140.4,136.9,134.8,132.5,127.0,124.2\left(d,{ }^{4} J_{C-F}=1.3\right), 120.1,116.5(d$, $\left.{ }^{3} J_{C-F}=6.6\right), 115.3,95.6\left(d,{ }^{1} J_{C-F}=198.0\right), 76.5,54.8,29.2,20.7,18.4$. Visible peaks of minor diastereoisomer: 161.5, 140.5, 136.7, 134.9, 132.1, 126.6, 124.4, 120.2, 115.4, 94.8 (d, ${ }^{1} \mathrm{~J}_{\mathrm{C}-\mathrm{F}}$ $=196.6), 75.8\left(\mathrm{~d},{ }^{2} \mathrm{~J}_{\mathrm{C}-\mathrm{F}}=23.8\right), 54.8,29.3,20.7,18.5 .{ }^{19} \mathrm{~F}$ NMR $\left(471 \mathrm{MHz}, \mathrm{C}_{6} \mathrm{D}_{6}, 25^{\circ} \mathrm{C}\right) \delta=-$ $198.72\left(d,{ }^{2} J_{H F}=47.7\right)$. Visible peaks of minor diastereoisomer: $-193.42\left(d,{ }^{2} J_{H F}=45.7\right)$. HRMS (ESI+): $\mathrm{m} / \mathrm{z}[\mathrm{M}+\mathrm{H}]^{+}$Calcd. for $\mathrm{C}_{20} \mathrm{H}_{21} \mathrm{FNO}_{4} \mathrm{~S}$ : 390.1170; Found: 390.1165.

\section{S-(4-methoxyphenyl) (R)-2-((R)-5,7-dibromo-3-hydroxy-1-methyl-2-oxoindolin-3-yl)-2- fluoroethanethioate $(4 v)$}

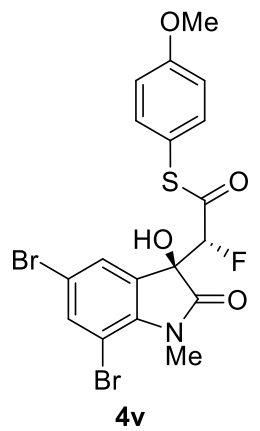

Prepared according to general procedure 2 using catalyst $F$. The product was purified by column chromatography (ethyl acetate / $n$-hexane) to afford the desired product as a white solid $(28.7 \mathrm{mg}, 90 \%$ yield, $2.0: 1 \mathrm{dr})$.

IR $\mathbf{V}_{\max }$ (neat)/cm ${ }^{-1} 3341,3074,2947,2837,2360,1717,1691,1591,1570$, 1493, 1454, 1340, 1291, 1247, 1174, 1104, 1078, 1026, 951, 867, 926, 774, 733, 707. ${ }^{1} \mathrm{H}$ NMR $\left(600 \mathrm{MHz}, \mathrm{C}_{6} \mathrm{D}_{6}, 25^{\circ} \mathrm{C}\right) \delta 7.37(\mathrm{~d}, J=1.9 \mathrm{~Hz}, 1 \mathrm{H}, \mathrm{ArH})$, $7.33(\mathrm{~d}, J=2.0 \mathrm{~Hz}, 1 \mathrm{H}, \mathrm{ArH}), 7.25-7.21(\mathrm{~m}, 2 \mathrm{H}, \mathrm{ArH}), 6.64-6.58(\mathrm{~m}, 2 \mathrm{H}$, $\mathrm{ArH}), 4.65(\mathrm{~d}, J=47.2 \mathrm{~Hz}, 1 \mathrm{H}, \mathrm{C}(\mathrm{F}) \mathrm{H}), 3.93(\mathrm{~d}, J=1.2 \mathrm{~Hz}, 1 \mathrm{H}, \mathrm{OH}), 3.11$ (s, $\left.3 \mathrm{H}, \mathrm{OCH}_{3}\right), 2.93\left(\mathrm{~s}, 3 \mathrm{H}, \mathrm{NCH}_{3}\right)$. Visible peaks of minor diastereoisomer 7.30 (d, $J=1.9 \mathrm{~Hz}, 1 \mathrm{H}, \mathrm{ArH}), 7.27(\mathrm{~d}, J=2.0 \mathrm{~Hz}, 1 \mathrm{H}, \mathrm{ArH}), 6.73-6.67(\mathrm{~m}, 2 \mathrm{H}, \mathrm{ArH}), 4.99$ (d, J = $46.0 \mathrm{~Hz}, 1 \mathrm{H}, \mathrm{C}(\mathrm{F}) \mathrm{H}), 3.68(\mathrm{~d}, J=1.2 \mathrm{~Hz}, 1 \mathrm{H}, \mathrm{OH}), 3.16\left(\mathrm{~s}, 3 \mathrm{H}, \mathrm{OCH}_{3}\right), 2.95\left(\mathrm{~s}, 3 \mathrm{H}, \mathrm{NCH}_{3}\right) .{ }^{13} \mathrm{C}$ $\operatorname{NMR}\left(151 \mathrm{MHz}, \mathrm{C}_{6} \mathrm{D}_{6}, 25^{\circ} \mathrm{C}\right) \delta 198.0,173.4,161.6,141.7,138.3,136.8,130.6,115.9,115.5$, 103.6, $94.4\left(\mathrm{~d},{ }^{1} \mathrm{~J}_{\mathrm{C}-\mathrm{F}}=199.0 \mathrm{~Hz}\right), 75.9\left(\mathrm{~d},{ }^{2} \mathrm{~J}_{\mathrm{C}-\mathrm{F}}=22.2 \mathrm{~Hz}\right), 54.8,29.5$. Visible peaks of minor diastereoisomer 197.8, 172.9, 172.8, 161.8, 141.7, 138.3, 136.7, 130.1, 115.8, 115.71, 115.6, 103.8, 94.4, 93.1, 75.8, 75.2, 54.9, 29.6. ${ }^{19} \mathrm{~F}$ NMR $\left(565 \mathrm{MHz}, \mathrm{C}_{6} \mathrm{D}_{6}, 25^{\circ} \mathrm{C}\right) \delta-194.28\left(\mathrm{~d},{ }^{2} J_{\mathrm{HF}}=\right.$ $46.1 \mathrm{~Hz}$ ). Visible peaks of minor diastereoisomer $-198.52\left(\mathrm{~d},{ }^{2} \mathrm{JFF}_{\mathrm{HF}}=47.2 \mathrm{~Hz}\right)$. HRMS (ESI+): $\mathrm{m} / \mathrm{z}[\mathrm{M}+\mathrm{Na}]^{+}$Calcd. for $\mathrm{C}_{18} \mathrm{H}_{14} \mathrm{Br}_{2} \mathrm{FNO}_{4} \mathrm{SNa}$ : 539.8887; Found: 539.8881 . 


\section{Methyl (R)-2-fluoro-2-((R)-3-hydroxy-1-methyl-2-oxoindolin-3-yl)acetate (5)}

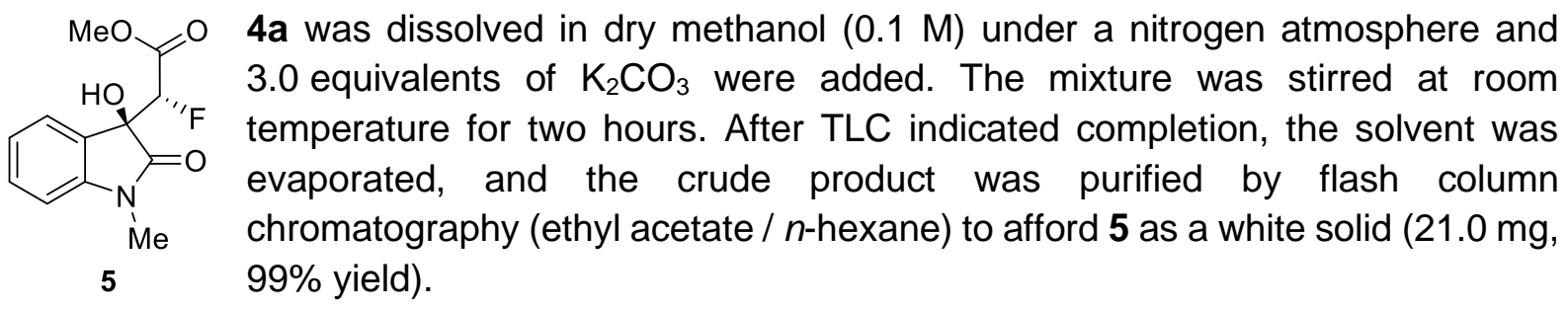

IR $\mathrm{v}_{\max }$ (neat)/cm ${ }^{-1} 3365,3059,2952,2933,2852,2359,1762,1708,1612,1494,1470,1374$, 1349, 1293, 1216, 1106, 1090, 1020, 801, 753, 692. ${ }^{1} \mathbf{H}$ NMR $\left(600 \mathrm{MHz}, \mathrm{CD}_{2} \mathrm{Cl}_{2}, 25^{\circ} \mathrm{C}\right) \delta 7.45$ (ddt, $J=7.4,1.3,0.6 \mathrm{~Hz}, 1 \mathrm{H}, \mathrm{ArH}), 7.41$ (td, $J=7.8,1.3 \mathrm{~Hz}, 1 \mathrm{H}, \mathrm{ArH}), \delta 7.15-7.12(\mathrm{~m}, 1 \mathrm{H}$, ArH), $6.90-6.87(\mathrm{~m}, 1 \mathrm{H}, \mathrm{ArH}), 5.22(\mathrm{~d}, J=47.0 \mathrm{~Hz}, 1 \mathrm{H}, \mathrm{C}(\mathrm{F}) \mathrm{H}), 3.72\left(\mathrm{~s}, 3 \mathrm{H}, \mathrm{OCH}_{3}\right), 3.17$ (s, $\left.3 \mathrm{H}, \mathrm{NH}_{3}\right)$. Visible peaks of minor diastereoisomer $7.43-7.40(\mathrm{~m}, 1 \mathrm{H}, \mathrm{ArH}), 7.28(\mathrm{ddd}, J=7.5$, 1.3, $0.6 \mathrm{~Hz}, 1 \mathrm{H}, \mathrm{ArH}), 7.13-7.07(\mathrm{~m}, 1 \mathrm{H}, \mathrm{ArH}), 6.92-6.89(\mathrm{~m}, 1 \mathrm{H}, \mathrm{ArH}), 5.18(\mathrm{~d}, J=46.4 \mathrm{~Hz}$, $1 \mathrm{H}, \mathrm{C}(\mathrm{F}) \mathrm{H}), 3.85\left(\mathrm{~s}, 3 \mathrm{H}, \mathrm{OCH}_{3}\right), 3.18\left(\mathrm{~s}, 3 \mathrm{H}, \mathrm{NCH}_{3}\right) .{ }^{13} \mathrm{C} \mathrm{NMR}\left(151 \mathrm{MHz}, \mathrm{CD}_{2} \mathrm{Cl}_{2}, 25^{\circ} \mathrm{C}\right) \delta 174.0$ $\left(\mathrm{d},{ }^{3} J_{\mathrm{C}-\mathrm{F}}=3.4 \mathrm{~Hz}\right), 167.8\left(\mathrm{~d},{ }^{2} \mathrm{~J}_{\mathrm{C}-\mathrm{F}}=23.0 \mathrm{~Hz}\right), 131.4,126.1,125.0,125.0,123.6,109.2,89.6$ (d, $\left.{ }^{1} J_{\mathrm{C}-\mathrm{F}}=196.6 \mathrm{~Hz}\right), 75.8\left(\mathrm{~d},{ }^{2} J_{\mathrm{C}-\mathrm{F}}=22.6 \mathrm{~Hz}\right), 53.2,26.6$. Visible peaks of minor diastereoisomer $173.9\left(\mathrm{~d},{ }^{3} \mathrm{~J}_{\mathrm{C}-\mathrm{F}}=2.0 \mathrm{~Hz}\right), 168.1\left(\mathrm{~d},{ }^{2} \mathrm{~J}_{\mathrm{C}-\mathrm{F}}=23.2 \mathrm{~Hz}\right), 144.9,131.4,125.7,125.07,123.5,109.3$, $89.2\left(\mathrm{~d},{ }^{1} J_{\mathrm{C}-\mathrm{F}}=193.3 \mathrm{~Hz}\right) ., 75.6\left(\mathrm{~d},{ }^{2} J_{\mathrm{C}-\mathrm{F}}=22.2 \mathrm{~Hz}\right), 53.3,26.8$. HRMS (ESI+): $\mathrm{m} / \mathrm{z}[\mathrm{M}+\mathrm{Na}]^{+}$ Calcd. for $\mathrm{C}_{12} \mathrm{H}_{12} \mathrm{FNO}_{4} \mathrm{Na}$ : 276.0643; Found: 276.0641 .

\section{(R)-N-benzyl-2-fluoro-2-((R)-3-hydroxy-1-methyl-2-oxoindolin-3-yl)acetamide (6)}

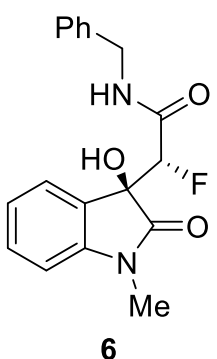

Under inert conditions 4a was dissolved in dry $\mathrm{CH}_{2} \mathrm{Cl}_{2}(0.1 \mathrm{M})$ and benzyl amine (3.0 equiv.) was added. The solution was stirred at room temperature for $5 \mathrm{~h}$ or until TLC indicated full conversion. The solvent was removed under reduced pressure and the crude product was purified by flash column chromatography (ethyl acetate / $n$-hexane) to afford 6 as a colorless oil $(27.2 \mathrm{mg}, 99 \%$ yield).

IR $\vee_{\max }$ (neat)/cm ${ }^{-1} 3345,3056,2927,2853,2339,1721,1661,1612,1543$, 1492, 1468, 1420, 1388, 1350, 1253, 1216, 1109, 1084, 1066, 1010, 951, 833, 795, 752, 710, 691. ${ }^{1} \mathrm{H}$ NMR $\left(600 \mathrm{MHz}, \mathrm{CD}_{2} \mathrm{Cl}_{2}, 25^{\circ} \mathrm{C}\right) \delta 7.40-7.32(\mathrm{~m}, 4 \mathrm{H}, \mathrm{ArH}), 7.31-7.28$ $(\mathrm{m}, 2 \mathrm{H}, \mathrm{ArH}), 7.08$ (ddd, $J=7.4,1.3,0.6 \mathrm{~Hz}, 1 \mathrm{H}, \mathrm{ArH}), 6.97-6.91(\mathrm{~m}, 1 \mathrm{H}, \mathrm{ArH}), 6.85$ (dt, $J=$ 7.8, $0.8 \mathrm{~Hz}, 1 \mathrm{H}, \mathrm{ArH}), 6.77$ (bs, $1 \mathrm{H}, \mathrm{NH}), 5.29$ (d, $J=46.7 \mathrm{~Hz}, 1 \mathrm{H}, \mathrm{C}(\mathrm{F}) \mathrm{H}), 4.61$ (dd, $J=14.8$, $6.6 \mathrm{~Hz}, 1 \mathrm{H}, \mathrm{ArCH}), 4.47$ (dd, $J=14.6,5.7 \mathrm{~Hz}, 1 \mathrm{H}, \mathrm{ArCH}), 3.16\left(\mathrm{~s}, 3 \mathrm{H}, \mathrm{NCH}_{3}\right)$. Visible peaks of minor diastereoisomer 7.45 (ddd, $J=7.4,1.5,0.5 \mathrm{~Hz}, 1 \mathrm{H}, \mathrm{ArH}), 7.41(\mathrm{td}, J=7.8,1.3 \mathrm{~Hz}, 1 \mathrm{H}$, ArH), $7.35-7.29(\mathrm{~m}, 2 \mathrm{H}, \mathrm{ArH}), 7.31-7.25(\mathrm{~m}, 1 \mathrm{H}, \mathrm{ArH}), 7.23-7.18(\mathrm{~m}, 2 \mathrm{H}, \mathrm{ArH}), 7.16-7.10$ $(\mathrm{m}, 1 \mathrm{H}, \mathrm{ArH}), 6.87(\mathrm{dt}, J=7.9,0.8 \mathrm{~Hz}, 1 \mathrm{H}, \mathrm{ArH}), 6.71(\mathrm{bs}, 1 \mathrm{H}, \mathrm{NH}), 5.21(\mathrm{~d}, J=47.2 \mathrm{~Hz}, 1 \mathrm{H}$, $\mathrm{C}(\mathrm{F}) \mathrm{H}), 4.55$ (dd, $J=14.9,6.4 \mathrm{~Hz}, 1 \mathrm{H}, \mathrm{ArCH}), 4.39$ (dd, $J=14.9,5.6 \mathrm{~Hz}, 1 \mathrm{H}, \mathrm{ArCH}), 3.15$ (s, $\left.3 \mathrm{H}, \mathrm{NCH}_{3}\right) .{ }^{13} \mathrm{C}$ NMR $\left(151 \mathrm{MHz}, \mathrm{CD}_{2} \mathrm{Cl}_{2}, 25^{\circ} \mathrm{C}\right) \delta 173.0,169.2\left(\mathrm{~d},{ }^{2} \mathrm{~J}_{\mathrm{C}-\mathrm{F}}=19.0 \mathrm{~Hz}\right), 145.1,137.2$, 131.0, 129.2, 128.5, 128.4, 126.5, 124.7, 123.4, 109.1, 88.7 (d, $\left.{ }^{1} J_{\mathrm{C}-\mathrm{F}}=195.3 \mathrm{~Hz}\right), 75.8\left(\mathrm{~d},{ }^{2} J_{\mathrm{C}-\mathrm{F}}\right.$ $=23.9 \mathrm{~Hz}), 43.6,26.7$. Visible peaks of minor diastereoisomer $174.4\left(\mathrm{~d},{ }^{3} \mathrm{~J}_{\mathrm{C}-\mathrm{F}}=2.3 \mathrm{~Hz}\right), 168.1$ $\left(\mathrm{d},{ }^{2} J_{\mathrm{C}-\mathrm{F}}=19.0 \mathrm{~Hz}\right), 145.3,137.6,131.2,129.1,128.1,128.0,126.7,124.8,124.8,123.5,109.1$, $90.0\left(\mathrm{~d},{ }^{1} \mathrm{~J}_{\mathrm{C}-\mathrm{F}}=196.3 \mathrm{~Hz}\right), 76.1\left(\mathrm{~d},{ }^{2} \mathrm{~J}_{\mathrm{C}-\mathrm{F}}=22.7 \mathrm{~Hz}\right), 43.3,26.6 .{ }^{19} \mathrm{~F}$ NMR $\left(565 \mathrm{MHz}, \mathrm{CD}_{2} \mathrm{Cl}_{2}\right.$, $\left.25^{\circ} \mathrm{C}\right) \delta-197.39\left(\mathrm{~d},{ }^{2} \mathrm{JF}_{\mathrm{HF}} \mathrm{J}=47.4 \mathrm{~Hz}\right.$ ). Visible peaks of minor diastereoisomer $-200.56\left(\mathrm{~d},{ }^{2} \mathrm{JF}_{\mathrm{HF}}\right.$ $=47.3 \mathrm{~Hz})$. HRMS (ESI+): $\mathrm{m} / \mathrm{z}[\mathrm{M}+\mathrm{Na}]^{+}$Calcd. for $\mathrm{C}_{18} \mathrm{H}_{17} \mathrm{FN}_{2} \mathrm{O}_{3} \mathrm{Na}: 351.1115$; Found: 351.1115 . 
(R)-3-((S)-1-fluoro-2-hydroxyethyl)-3-hydroxy-1-methylindolin-2-one (7)

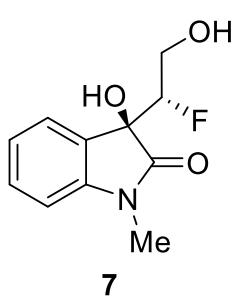

Under inert conditions $4 \mathrm{a}$ was dissolved in dry THF $(0.1 \mathrm{M})$ and the solution was cooled to $0{ }^{\circ} \mathrm{C}$. Subsequently, $\mathrm{NaBH}_{4}$ (5.0 equiv.) was added in one portion and the solution was stirred at $0{ }^{\circ} \mathrm{C}$ for $30 \mathrm{~min}$. Then the solution was allowed to warm up to room temperature and stirred for additional $30 \mathrm{~min}$ at this temperature. The reaction was quenched with $0.1 \mathrm{M} \mathrm{HCl}$ and extracted with ethyl acetate. The combined organic phases were dried over $\mathrm{Na}_{2} \mathrm{SO}_{4}$ and concentrated under reduced pressure. The crude product was purified by flash column chromatography (ethyl acetate / $n$-hexane) to afford 7 as a white solid (22.2 mg, $85 \%$ yield).

IR $\mathrm{v}_{\max }$ (neat) $/ \mathrm{cm}^{-1} 3355,2929,1703,1613,1494,1470,1376,1351,1258,1229,1114,1093$, 1047, 1021, 938, 872, 754, 707. ' $\mathrm{H}$ NMR $\left(500 \mathrm{MHz}, \mathrm{CD}_{2} \mathrm{Cl}_{2}, 25^{\circ} \mathrm{C}\right) \delta 7.45$ (ddt, $J=1.5,1.0$, $0.6 \mathrm{~Hz}, 1 \mathrm{H}, \mathrm{ArH}), 7.40$ (td, $J=7.8,1.3 \mathrm{~Hz}, 1 \mathrm{H}, \mathrm{ArH}), 7.18-7.10(\mathrm{~m}, 1 \mathrm{H}, \mathrm{ArH}), 6.90$ (dt, $J=$ 7.8, $0.5 \mathrm{~Hz}, 1 \mathrm{H}, \mathrm{ArH}$ ), 4.60 (ddd, $J=45.8,4.6,3.5 \mathrm{~Hz}, 1 \mathrm{H}, \mathrm{C}(\mathrm{F}) \mathrm{H}), 4.05$ (d, J=24.9 Hz, 2H, $\left.\mathrm{CH}_{2}\right), 3.17\left(\mathrm{~s}, 3 \mathrm{H}, \mathrm{NCH}_{3}\right)$. Visible peaks of minor diastereoisomer $7.46(\mathrm{td}, J=1.3,0.6 \mathrm{~Hz}, 1 \mathrm{H}$, ArH), 7.41 (td, $J=7.8,1.3 \mathrm{~Hz}, 1 \mathrm{H}, \mathrm{ArH}$ ), 7.15 (ddd, $J=7.4,1.0 \mathrm{~Hz}, 1 \mathrm{H}, \mathrm{ArH}$ ), 4.85 (ddd, $J=$ 47.3, 5.9, 4.2 Hz, $1 \mathrm{H}, \mathrm{C}(\mathrm{F}) \mathrm{H}), 4.01\left(\mathrm{~d}, J=15.3 \mathrm{~Hz}, 2 \mathrm{H}, \mathrm{CH}_{2}\right) .{ }^{13} \mathrm{C}$ NMR (126 MHz, $\mathrm{CD}_{2} \mathrm{Cl}_{2}$, $\left.25^{\circ} \mathrm{C}\right) \delta 175.7\left(\mathrm{~d},{ }^{3} \mathrm{~J}_{\mathrm{C}-\mathrm{F}}=5.8 \mathrm{~Hz}\right), 130.9,125.8,125.8,124.9,124.9,123.6,109.2,94.2\left(\mathrm{~d},{ }^{1} \mathrm{~J}_{\mathrm{C}-\mathrm{F}}\right.$ $=183.1 \mathrm{~Hz}), 77.2\left(\mathrm{~d},{ }^{2} \mathrm{~J}_{\mathrm{C}-\mathrm{F}}=21.3 \mathrm{~Hz}\right), 61.8\left(\mathrm{~d},{ }^{2} J_{\mathrm{C}-\mathrm{F}}=22.6 \mathrm{~Hz}\right), 26.7$. Visible peaks of minor diastereoisomer 144.5, 131.0, 127.7, 127.5, 123.8, 109.3, $94.7\left(\mathrm{~d},{ }^{1} \mathrm{~J}_{\mathrm{C}-\mathrm{F}}=180.3 \mathrm{~Hz}\right), 76.7$ (d, $\left.{ }^{2} J_{\mathrm{C}-\mathrm{F}}=23.7 \mathrm{~Hz}\right), 61.4\left(\mathrm{~d},{ }^{2} \mathrm{~J}_{\mathrm{C}-\mathrm{F}}=23.8 \mathrm{~Hz}\right), 26.7 .{ }^{19} \mathrm{~F} \mathbf{N M R}\left(471 \mathrm{MHz}, \mathrm{CD}_{2} \mathrm{Cl}_{2}, 25^{\circ} \mathrm{C}\right) \delta-200.55$ (ddd, $\left.{ }^{2} J_{\mathrm{HF}}=45.9,26.4,23.8 \mathrm{~Hz}\right)$. Visible peaks of minor diastereoisomer -202.03 (ddd, ${ }^{2} J_{\mathrm{HF}}=$ 47.4, 23.7, $20.5 \mathrm{~Hz}$ ). HRMS (ESI+): $\mathrm{m} / \mathrm{z}[\mathrm{M}+\mathrm{Na}]^{+}$Calcd. for $\mathrm{C}_{11} \mathrm{H}_{12} \mathrm{FNO}_{3} \mathrm{Na}$ : 248.0693; Found: 248.0691 .

\section{(R)-3-((R)-1-fluoro-2-(4-methoxyphenyl)-2-oxoethyl)-3-hydroxy-1-methylindolin-2-one}

(8)

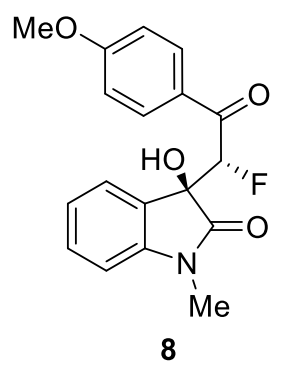

Under inert conditions $\mathbf{4 a}, \mathrm{Cu}(\mathrm{I})$ thiophene-2-carboxylate (1.6 equiv.), (4methoxyphenyl)boronic acid (1.2 equiv.), $\mathrm{Pd}_{2} \mathrm{dba}_{3}$ (5 mol\%) and tri(furan-2yl)phosphane (15 mol\%) were added to a Schlenk-flask. The solids were dissolved in dry THF $(0.1 \mathrm{M})$ and heated at $50^{\circ} \mathrm{C}$ in an oil bath for $20 \mathrm{~h}$. The reaction was quenched by the addition of $1 \mathrm{M} \mathrm{HCl}(5 \mathrm{~mL})$ and then $5 \mathrm{~mL}$ diethyl ether was added. The phases were separated, and the organic phase was washed with water $(5 \mathrm{~mL})$. The organic layer was dried over $\mathrm{Na}_{2} \mathrm{SO}_{4}$ and concentrated under reduced pressure. The crude product was purified by flash column chromatography (ethyl acetate / $n$-hexane) to afford 8 as a white solid (26.4 $\mathrm{mg}, 64 \%$ yield).

IR $\mathbf{v}_{\max }$ (neat)/cm ${ }^{-1} 3353,3059,2932,2843,2357,1706,1682,1598,1572,1510,1494,1470$, 1422, 1351, 1310, 1261, 1244, 1174, 1091, 1021, 957, 841, 754. ${ }^{1} \mathrm{H}$ NMR (500 MHz, $\mathrm{C}_{6} \mathrm{D}_{6}$, $\left.25^{\circ} \mathrm{C}\right) \delta 7.87-7.81(\mathrm{~m}, 2 \mathrm{H}, \mathrm{ArH}), 7.48-7.43(\mathrm{~m}, 1 \mathrm{H}, \mathrm{ArH}), 6.96(\mathrm{td}, J=7.8,1.3 \mathrm{~Hz}, 1 \mathrm{H}, \mathrm{ArH})$, $6.78(\mathrm{td}, J=7.5,1.0 \mathrm{~Hz}, 1 \mathrm{H}, \mathrm{ArH}), 6.49-6.43(\mathrm{~m}, 2 \mathrm{H}, \mathrm{ArH}), 6.22-6.17(\mathrm{~m}, 1 \mathrm{H}, \mathrm{ArH}), 5.77(\mathrm{~d}$, $J=47.1 \mathrm{~Hz}, 1 \mathrm{H}, \mathrm{C}(\mathrm{F}) \mathrm{H}), 3.06\left(\mathrm{~s}, 3 \mathrm{H}, \mathrm{OCH}_{3}\right), 2.59\left(\mathrm{~s}, 3 \mathrm{H}, \mathrm{NCH}_{3}\right)$. Visible peaks of minor diastereoisomer 7.70 (dd, $J=3.7,1.3 \mathrm{~Hz}, 2 \mathrm{H}, \mathrm{ArH}), 6.78-6.74(\mathrm{~m}, 2 \mathrm{H}, \mathrm{ArH}), 6.16$ (ddt, $J=$ 7.8, 1.5, $0.7 \mathrm{~Hz}, 1 \mathrm{H}, \mathrm{ArH}), 5.99(\mathrm{dd}, J=46.1,1.6 \mathrm{~Hz}, 1 \mathrm{H}, \mathrm{C}(\mathrm{F}) \mathrm{H}), 3.08(\mathrm{~d}, J=0.6 \mathrm{~Hz}, 3 \mathrm{H}$, $\left.\mathrm{OCH}_{3}\right), 2.55\left(\mathrm{~d}, J=0.9 \mathrm{~Hz}, 3 \mathrm{H}, \mathrm{NCH}_{3}\right) .{ }^{13} \mathrm{C}$ NMR $\left(126 \mathrm{MHz}, \mathrm{C}_{6} \mathrm{D}_{6}, 2^{\circ} \mathrm{C}\right) \delta 193.9\left(\mathrm{~d},{ }^{2} \mathrm{~J}_{\mathrm{C}-\mathrm{F}}=\right.$ 
$21.3 \mathrm{~Hz}), 174.35\left(\mathrm{~d},{ }^{3} \mathrm{~J}_{\mathrm{C}-\mathrm{F}}=5.5 \mathrm{~Hz}\right), 163.9,144.8,134.4,133.0,132.0,131.9,129.9,124.9$, 124.9, 122.6, 113.5, 108.2, $95.5\left(\mathrm{~d},{ }^{1} J_{\mathrm{C}-\mathrm{F}}=194.8 \mathrm{~Hz}\right), 76.6\left(\mathrm{~d},{ }^{2} J_{\mathrm{C}-\mathrm{F}}=21.6 \mathrm{~Hz}\right), 54.5,25.4$. Visible peaks of minor diastereoisomer 165.6, 132.0, 130.1, 128.0, 127.9, 127.7, 127.5, 122.5, 113.7, 108.3, 54.5, 25.5. ${ }^{19} \mathrm{~F}$ NMR $\left(471 \mathrm{MHz}, \mathrm{C}_{6} \mathrm{D}_{6}, 25^{\circ} \mathrm{C}\right) \delta-196.10\left(\mathrm{~d},{ }^{2} \mathrm{JHF}_{\mathrm{HF}}=46.4 \mathrm{~Hz}\right)$. Visible peaks of minor diastereoisomer -191.45 (dd, $J=46.0,16.8 \mathrm{~Hz}$ ). HRMS (ESI+): m/z [M+Na] ${ }^{+}$ Calcd. for $\mathrm{C}_{18} \mathrm{H}_{16} \mathrm{FNO}_{4} \mathrm{Na}$ : 352.0956; Found: 352.0953.

\section{(S)-4,7-dichloro-3-((S)-1-fluoro-2-(4-methoxyphenyl)-2-oxoethyl)-3-hydroxyindolin-2- one (9)}<smiles>COc1ccc(C(=O)C(F)C2(O)C(=O)Nc3c(Cl)ccc(Cl)c32)cc1</smiles>

Under inert conditions 40 was dissolved in dry THF $(0.1 \mathrm{M})$. The solution was cooled to $0{ }^{\circ} \mathrm{C}$ and (4-methoxyphenyl)magnesium bromide ( 3.5 equiv., $0.5 \mathrm{M}$ in THF) was added dropwise at this temperature. The solution was stirred for ten minutes at $0{ }^{\circ} \mathrm{C}$ and then warmed up to room temperature and stirred for additional $20 \mathrm{~min}$. The reaction was quenched by the addition of $0.1 \mathrm{M} \mathrm{HCl}$. The phases were separated and the aqueous phase was extracted with $\mathrm{CH}_{2} \mathrm{Cl}_{2}$ three times. The combined organic layers were dried of $\mathrm{Na}_{2} \mathrm{SO}_{4}$ and concentrated under reduced pressure. The crude product was purified by flash column chromatography (ethyl acetate / $n$-hexane) to afford 9 as a white solid $(7.5 \mathrm{mg}, 60 \%$ yield).

IR $\mathrm{v}_{\max }$ (neat) $/ \mathrm{cm}^{-1} 3307,2956,2921,2849,2359,2339,1738,1677,1614,1598,1572,1511$, $1469,1423,1311,1262,1240,1173,1117,1084,1026,968,930,838,802,744 .{ }^{1} \mathbf{H}$ NMR (400 MHz, $\left.\mathrm{CD}_{2} \mathrm{Cl}_{2}, 25^{\circ} \mathrm{C}\right) \delta 8.04-7.96(\mathrm{~m}, 2 \mathrm{H}, \mathrm{ArH}), 7.65(\mathrm{bs}, 1 \mathrm{H}, \mathrm{NH}), 7.30(\mathrm{~d}, J=8.7 \mathrm{~Hz}$, $1 \mathrm{H}, \mathrm{ArH}), 7.05(\mathrm{~d}, J=8.8 \mathrm{~Hz}, 1 \mathrm{H}, \mathrm{ArH}), 6.99-6.94(\mathrm{~m}, 2 \mathrm{H}, \mathrm{ArH}), 6.10(\mathrm{~d}, J=46.4 \mathrm{~Hz}, 1 \mathrm{H}$, $\mathrm{C}(\mathrm{F}) \mathrm{H}), 3.88\left(\mathrm{~s}, 3 \mathrm{H}, \mathrm{OCH}_{3}\right)$. Visible peaks of minor diastereoisomer $7.93-7.85(\mathrm{~m}, 2 \mathrm{H}, \mathrm{ArH})$, $7.19(\mathrm{~d}, J=8.8 \mathrm{~Hz}, 1 \mathrm{H}, \mathrm{ArH}), 6.96-6.88(\mathrm{~m}, 2 \mathrm{H}, \mathrm{ArH}), 6.88(\mathrm{~d}, J=8.8 \mathrm{~Hz}, 1 \mathrm{H}, \mathrm{ArH}), 6.34(\mathrm{~d}$, $J=46.7 \mathrm{~Hz}, 1 \mathrm{H}, \mathrm{C}(\mathrm{F}) \mathrm{H}), 3.86\left(\mathrm{~s}, 3 \mathrm{H}, \mathrm{OCH}_{3}\right) .{ }^{13} \mathrm{C}$ NMR $\left(151 \mathrm{MHz}, \mathrm{CD}_{2} \mathrm{Cl}_{2}, 25^{\circ} \mathrm{C}\right) \delta 196.7(\mathrm{~d}$, $\left.{ }^{2} J_{C-F}=20.7 \mathrm{~Hz}\right), 174.1,165.2,141.5,132.6,132.1,130.7,128.9,128.6\left(\mathrm{~d},{ }^{3} J_{C-F}=3.6 \mathrm{~Hz}\right)$, 125.4, 124.9, 114.7, 114.3, 114.3, $91.1\left(\mathrm{~d},{ }^{1} \mathrm{~J}_{\mathrm{C}-\mathrm{F}}=194.9 \mathrm{~Hz}\right), 79.6\left(\mathrm{~d},{ }^{2} \mathrm{~J}_{\mathrm{C}-\mathrm{F}}=26.4 \mathrm{~Hz}\right), 56.1$. Visible peaks of minor diastereoisomer $191.8\left(\mathrm{~d},{ }^{2} \mathrm{~J}_{\mathrm{C}-\mathrm{F}}=19.1 \mathrm{~Hz}\right), 174.1,165.1,141.3,132.6$, 132.1, 132.0, 130.7, $128.6\left(\mathrm{~d},{ }^{3} J_{\mathrm{C}-\mathrm{F}}=3.6 \mathrm{~Hz}\right), 125.1,114.6,114.4,91.9\left(\mathrm{~d},{ }^{1} J_{\mathrm{C}-\mathrm{F}}=194.6 \mathrm{~Hz}\right)$, $77.7\left(\mathrm{~d},{ }^{2} J_{\mathrm{C}-\mathrm{F}}=21.2 \mathrm{~Hz}\right), 56.1 .{ }^{19} \mathrm{~F}$ NMR $\left(376 \mathrm{MHz}, \mathrm{CD}_{2} \mathrm{Cl}_{2}, 25^{\circ} \mathrm{C}\right) \delta-191.92\left(\mathrm{~d},{ }^{2} J_{\mathrm{HF}}=46.4 \mathrm{~Hz}\right)$. Visible peaks of minor diastereoisomer $-197.65\left(\mathrm{~d},{ }^{2} \mathrm{JHF}_{\mathrm{HF}}=46.6 \mathrm{~Hz}\right)$. HRMS (ESI+): $\mathrm{m} / \mathrm{z}[\mathrm{M}+\mathrm{Na}]^{+}$ Calcd. for $\mathrm{C}_{17} \mathrm{H}_{12} \mathrm{Cl}_{2} \mathrm{FNO}_{4} \mathrm{Na}$ : 406.0020; Found: 406.0019 . 


\section{Crystal Structure Analysis of Fluorinated Oxindoles}

Crystal Structure of S-(4-methoxyphenyl) (R)-2-((R)-1-benzyl-4,7-dichloro-3-hydroxy-2oxoindolin-3-yl)-2-fluoroethanethioate (4p)

Single crystals of $4 p$ were obtained by liquid-liquid diffusion in benzene and $n$-hexane as the anti-solvent. A suitable crystal was selected and measured on a XtaLAB Synergy, Dualflex, Pilatus 300K diffractometer. The crystal was kept at 100.0(1) K during data collection. Using Olex $2^{8}$, the structure was solved with the ShelXT ${ }^{9}$ structure solution program using Intrinsic Phasing and refined with the SheIXL ${ }^{10}$ refinement package using Least Squares minimization.

Crystal size $/ \mathrm{mm}^{3}: 0.194 \times 0.117 \times 0.072$

Crystal Data for $\mathrm{C}_{27} \mathrm{H}_{21} \mathrm{Cl}_{2} \mathrm{FNO}_{4} \mathrm{~S}(\mathrm{M}=545.41 \mathrm{~g} / \mathrm{mol})$ : triclinic, space group P-1 (no. 2), $\mathrm{a}=$ $10.11410(10) \AA, b=11.02490(10) \AA, c=12.92480(10) \AA, \alpha=112.9100(10)^{\circ}, \beta=$ $105.7200(10)^{\circ}, y=96.9710(10)^{\circ}, V=1235.34(2) \AA^{3}, Z=2, T=100.0(1) K, \mu($ CuKa $)=3.526$ $\mathrm{mm}^{-1}, \mathrm{D}_{\text {calc }}=1.466 \mathrm{~g} / \mathrm{cm}^{3}, 47155$ reflections measured $\left(7.922^{\circ} \leq 2 \Theta \leq 159.37^{\circ}\right), 5250$ unique $\left(R_{\text {int }}=0.0366, R_{\text {sigma }}=0.0184\right)$ which were used in all calculations. The final $R_{1}$ was $0.0286(I$ $>2 \sigma(\mathrm{l}))$ and $w \mathrm{R}_{2}$ was 0.0744 (all data).

The crystal structure is deposited in the Cambridge Crystallographic Data Centre (CCDC Code: 2049349).

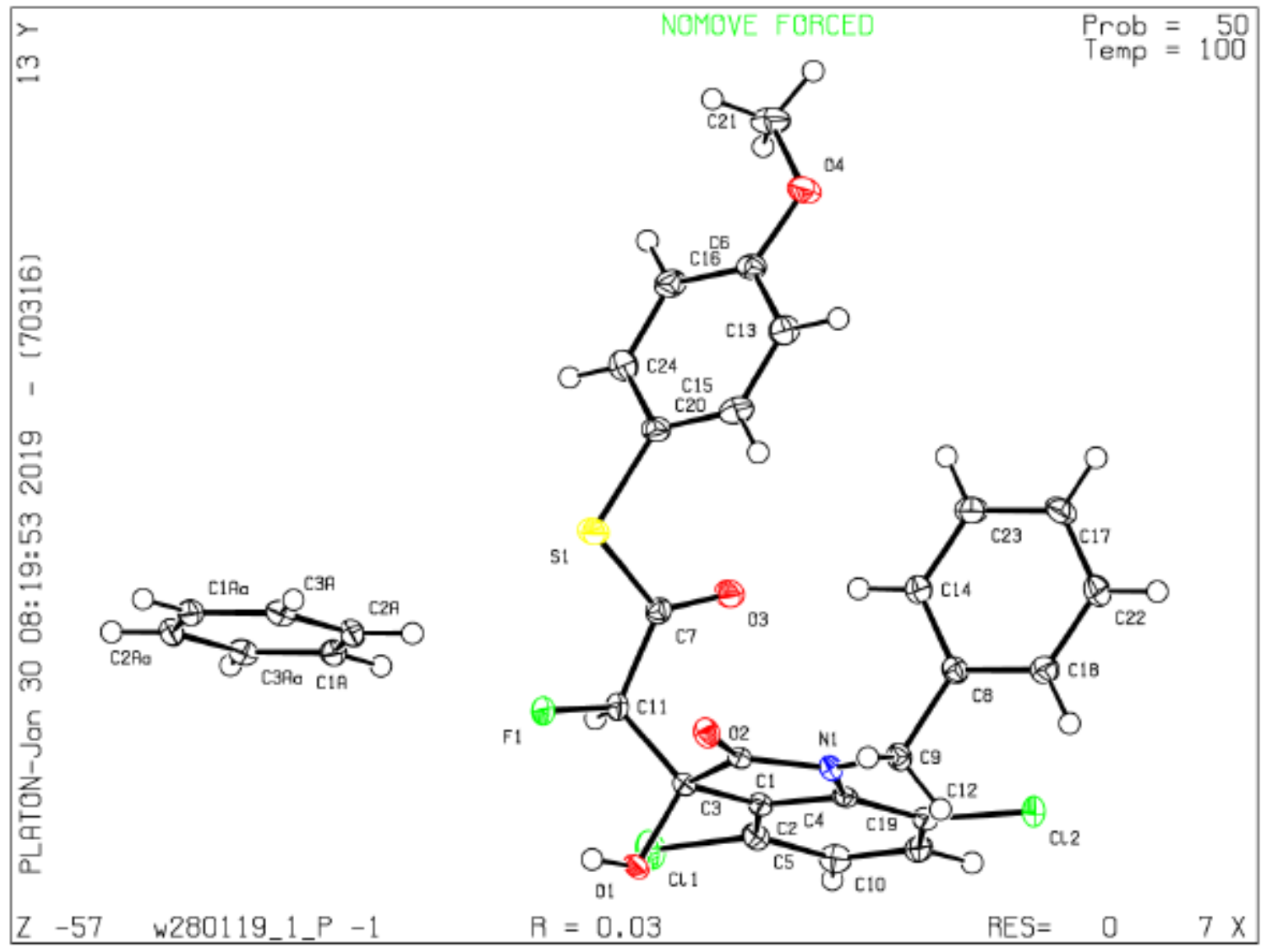


Crystal Structure of S-(4-methoxyphenyl) (R)-2-fluoro-2-((R)-3-hydroxy-1-methyl-2-oxo-4phenylindolin-3-yl)ethanethioate (4m)

Single crystals of $\mathbf{4 m}$ were obtained by liquid-liquid diffusion in benzene and $n$-hexane as the anti-solvent. A suitable crystal was selected and measured on a XtaLAB Synergy, Dualflex, Pilatus 300K diffractometer. The crystal was kept at 100.0(1) K during data collection. Using Olex $2^{8}$, the structure was solved with the ShelXT ${ }^{9}$ structure solution program using Intrinsic Phasing and refined with the ShelXL ${ }^{10}$ refinement package using Least Squares minimization.

Crystal size $/ \mathrm{mm}^{3}: 0.285 \times 0.176 \times 0.055$

Crystal Data for $\mathrm{C}_{24} \mathrm{H}_{20} \mathrm{FNO} 4 \mathrm{~S}(\mathrm{M}=437.47 \mathrm{~g} / \mathrm{mol}$ ): triclinic, space group P-1 (no. 2), $\mathrm{a}=$ $9.48400(10) \AA, b=9.84270(10) \AA, c=11.39790(10) \AA, \alpha=97.5380(10)^{\circ}, \beta=102.9740(10)^{\circ}$, $\mathrm{V}=92.8890(10)^{\circ}, \mathrm{V}=1024.334(18) \AA^{3}, \mathrm{Z}=2, \mathrm{~T}=100.0(1) \mathrm{K}, \mu(\mathrm{Cu} \mathrm{K \alpha})=1.763 \mathrm{~mm}^{-1}, \mathrm{D}_{\text {calc }}=$ $1.418 \mathrm{~g} / \mathrm{cm}^{3}, 25511$ reflections measured $\left(8.046^{\circ} \leq 2 \Theta \leq 159.668^{\circ}\right), 4317$ unique $\left(R_{\text {int }}=0.0323\right.$, $\left.R_{\text {sigma }}=0.0198\right)$ which were used in all calculations. The final $R_{1}$ was $0.0321(I>2 \sigma(I))$ and $w \mathrm{R}_{2}$ was 0.0836 (all data).

The crystal structure is deposited in the Cambridge Crystallographic Data Centre (CCDC Code: 2056442).

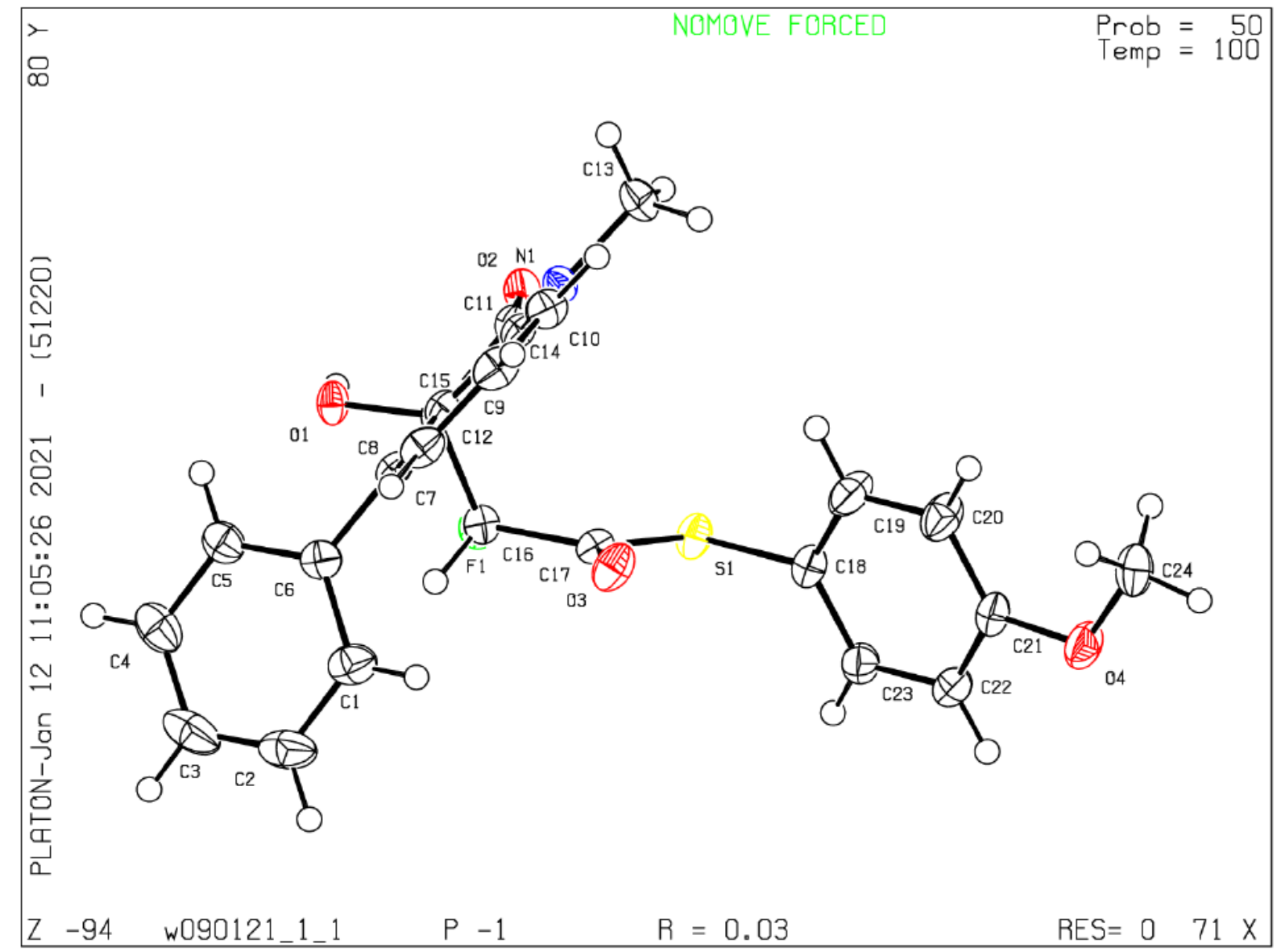




\section{NMR Spectra}

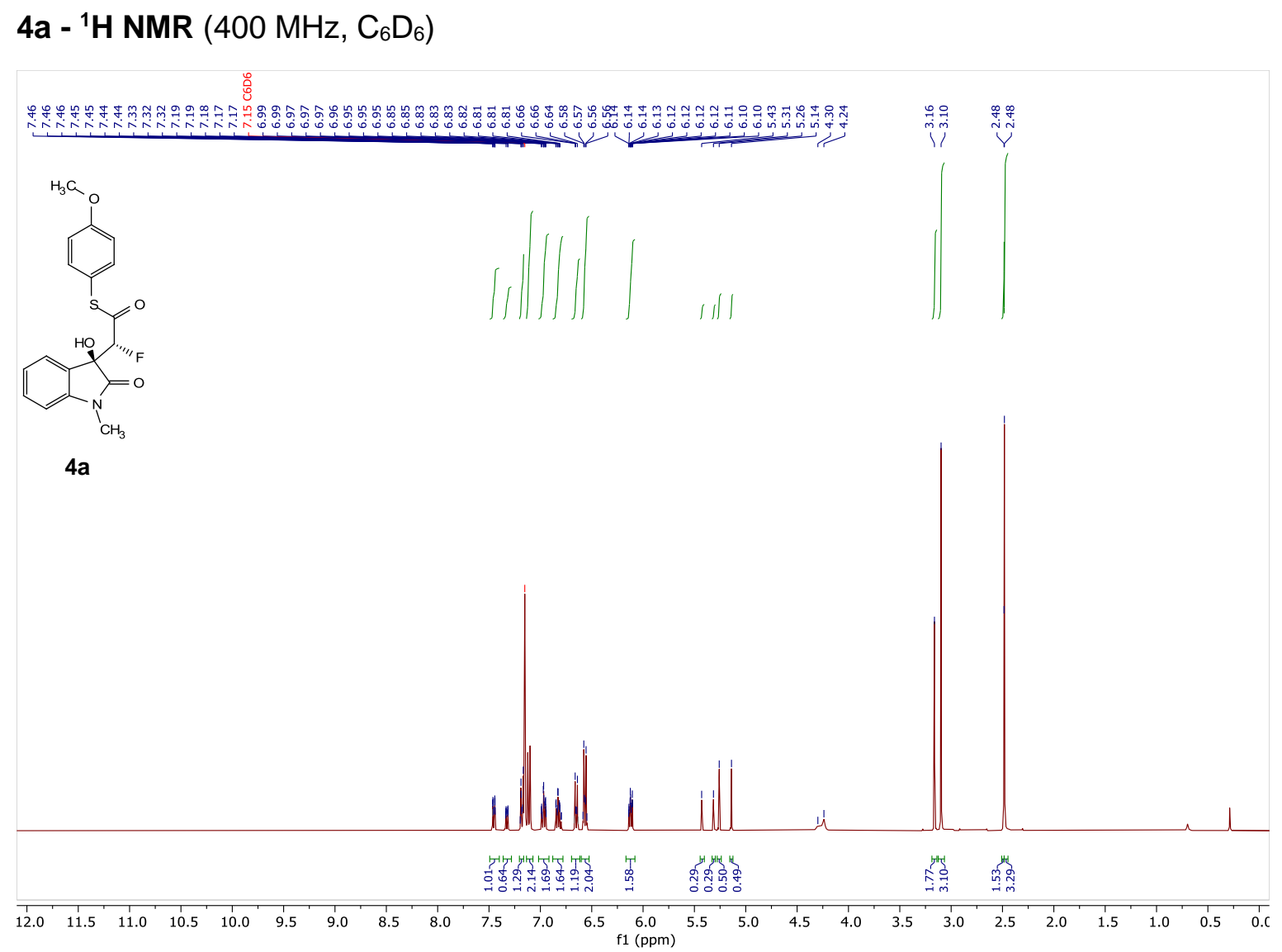

4a $-{ }^{13} \mathrm{C}$ NMR $\left(101 \mathrm{MHz}, \mathrm{C}_{6} \mathrm{D}_{6}\right)$

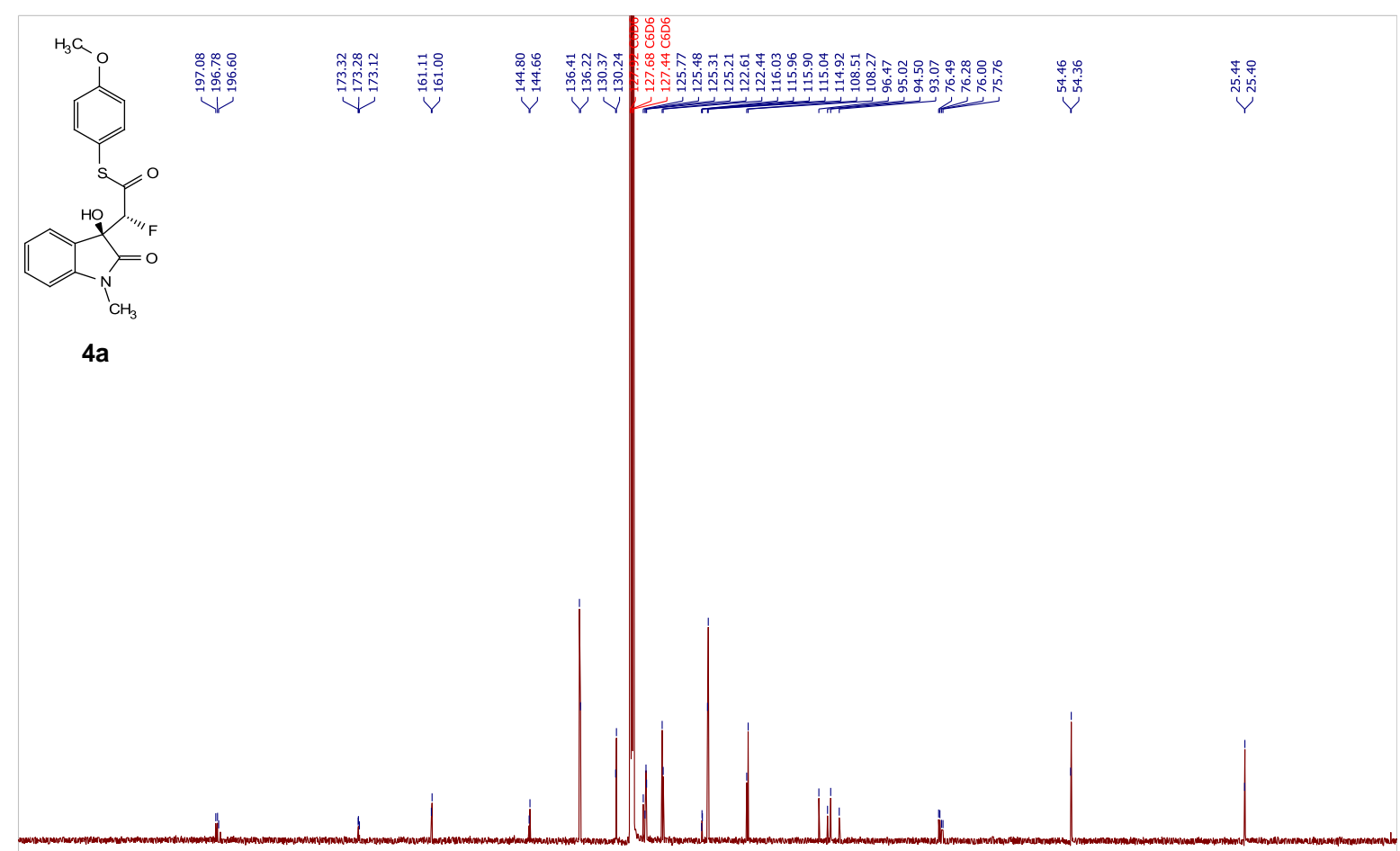

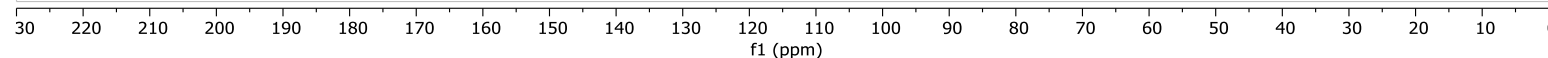


Aa - ${ }^{19}$ F NMR $\left(377 \mathrm{MHz}, \mathrm{C}_{6} \mathrm{D}_{6}\right)$

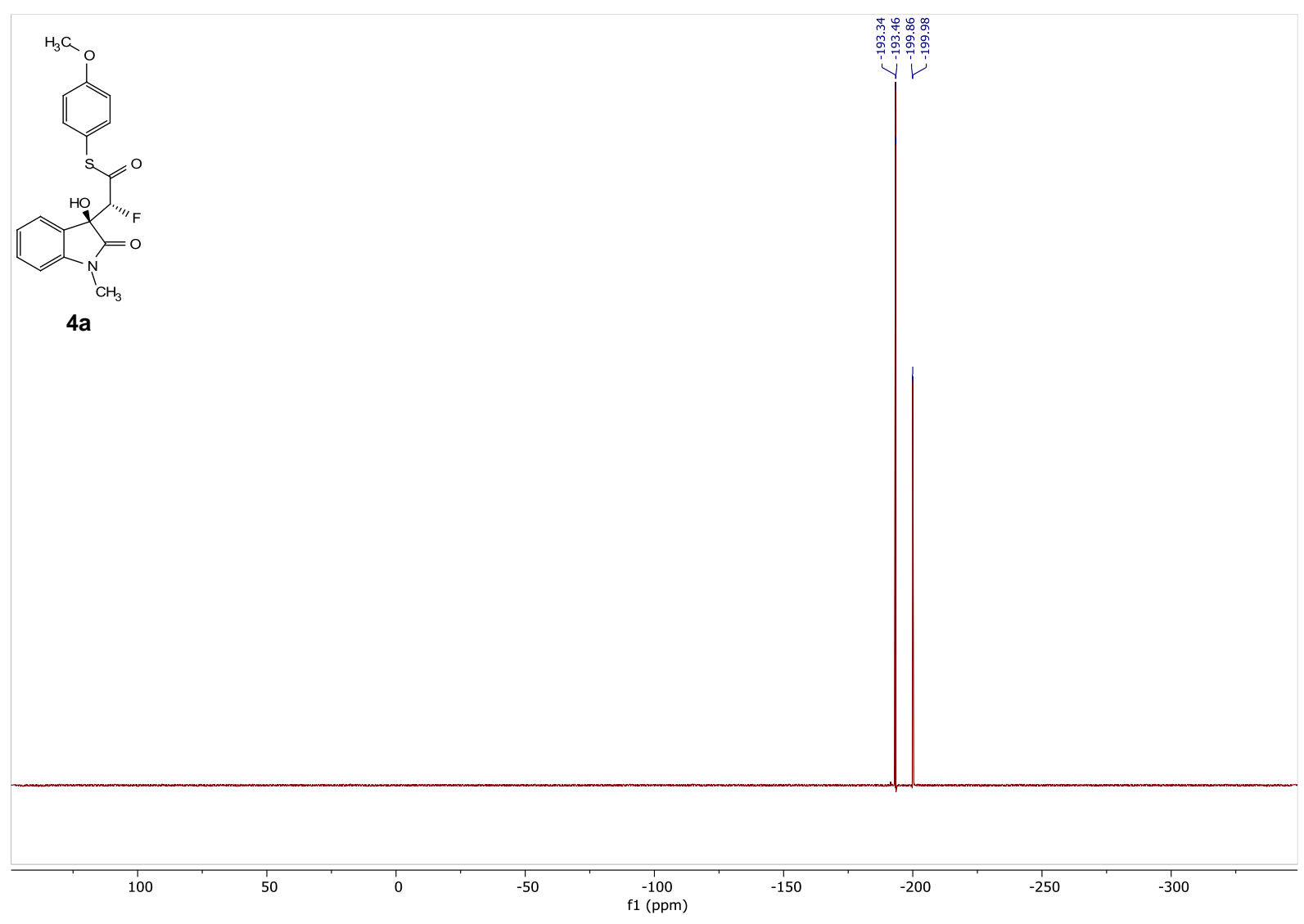

Ab - ${ }^{1} \mathbf{H}$ NMR $\left(400 \mathrm{MHz}, \mathrm{CD}_{2} \mathrm{Cl}_{2}\right)$

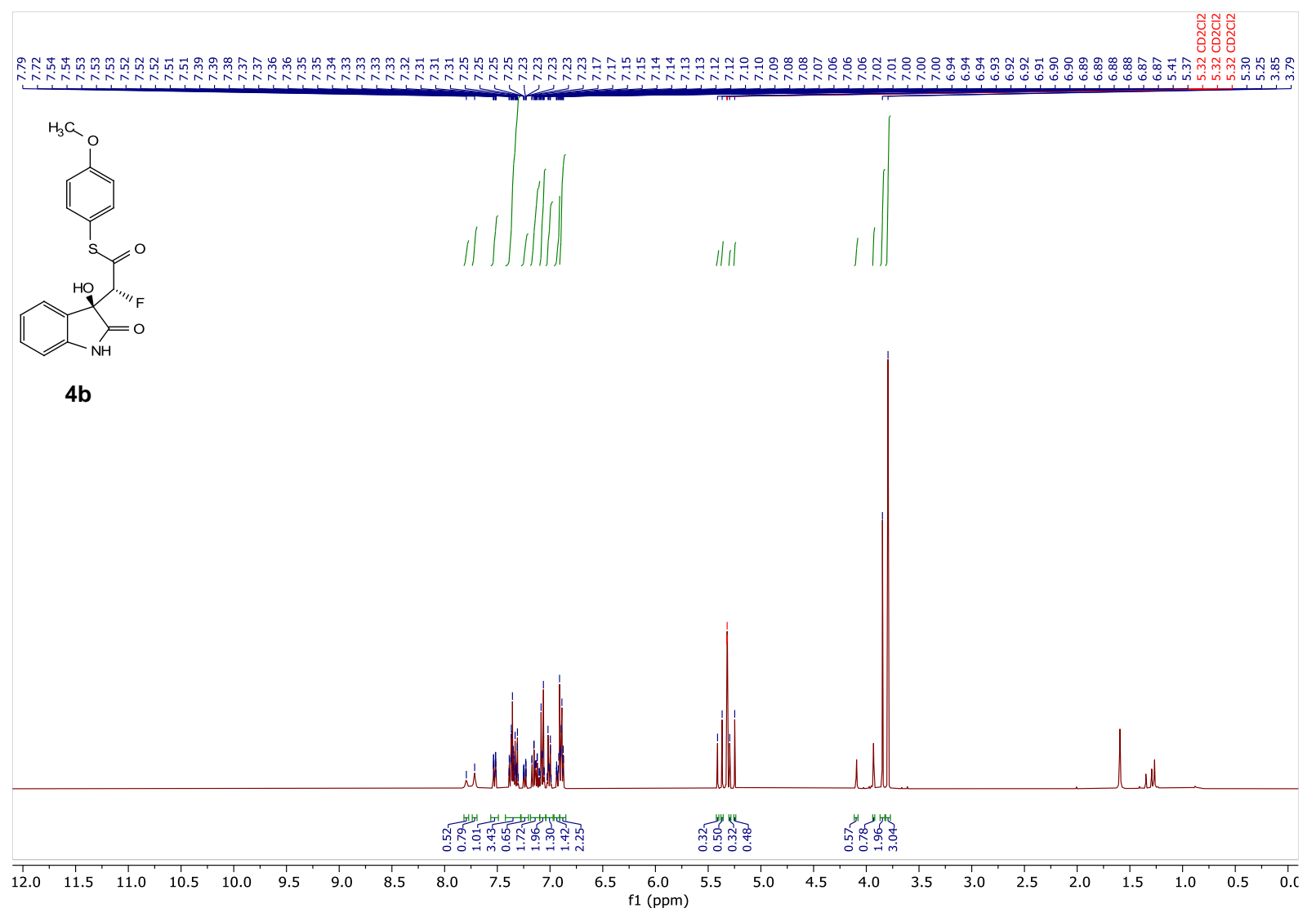


4b $-{ }^{13} \mathbf{C}$ NMR $\left(101 \mathrm{MHz}, \mathrm{CD}_{2} \mathrm{Cl}_{2}\right)$
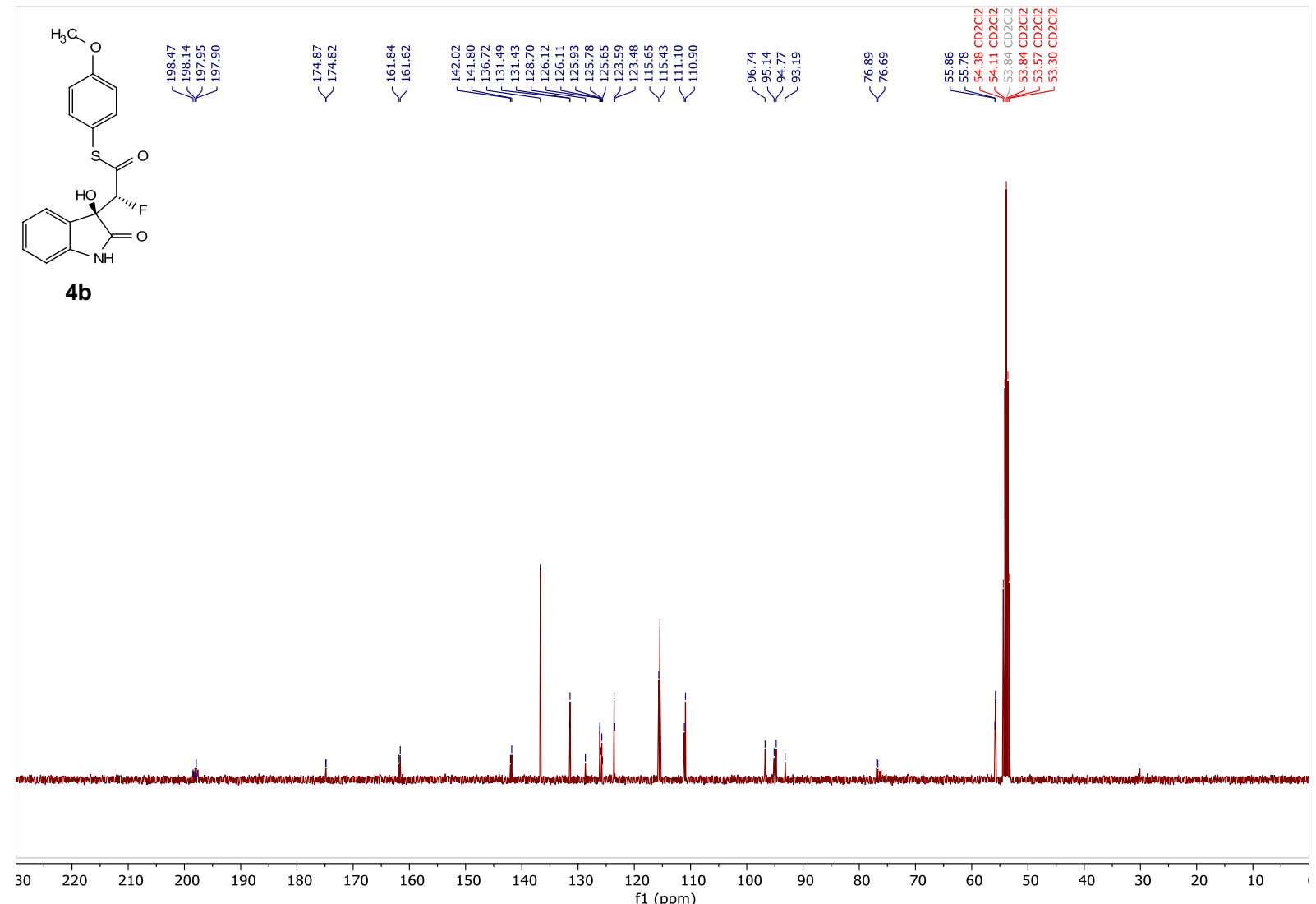

4b - ${ }^{19}$ F NMR (376 MHz, $\mathrm{CD}_{2} \mathrm{Cl}_{2}$ )

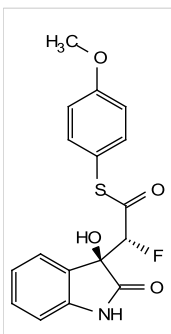

$4 b$ 


\section{4c $-{ }^{1} \mathrm{H}$ NMR $\left(400 \mathrm{MHz}, \mathrm{C}_{6} \mathrm{D}_{6}\right)$}

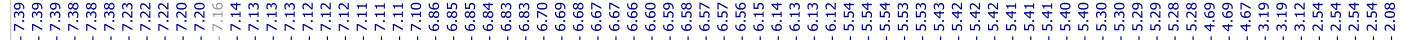

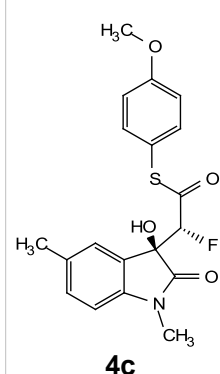
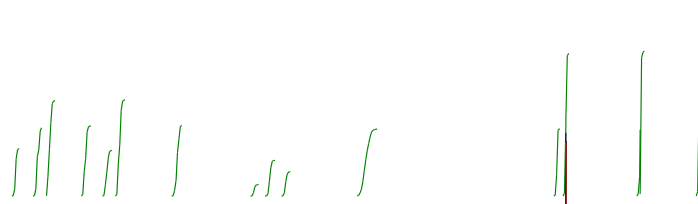

$4 \mathrm{c}$

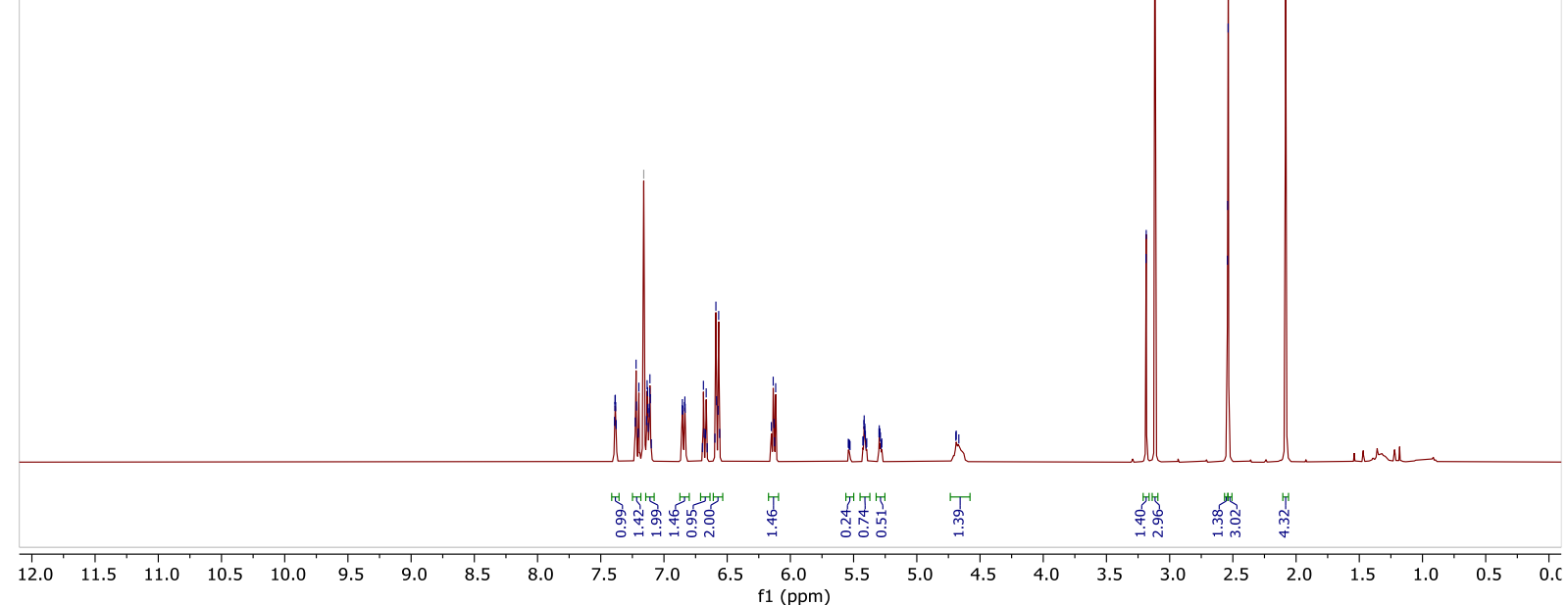

4c - ${ }^{13}$ C NMR (101 MHz, $\left.\mathrm{C}_{6} \mathrm{D}_{6}\right)$

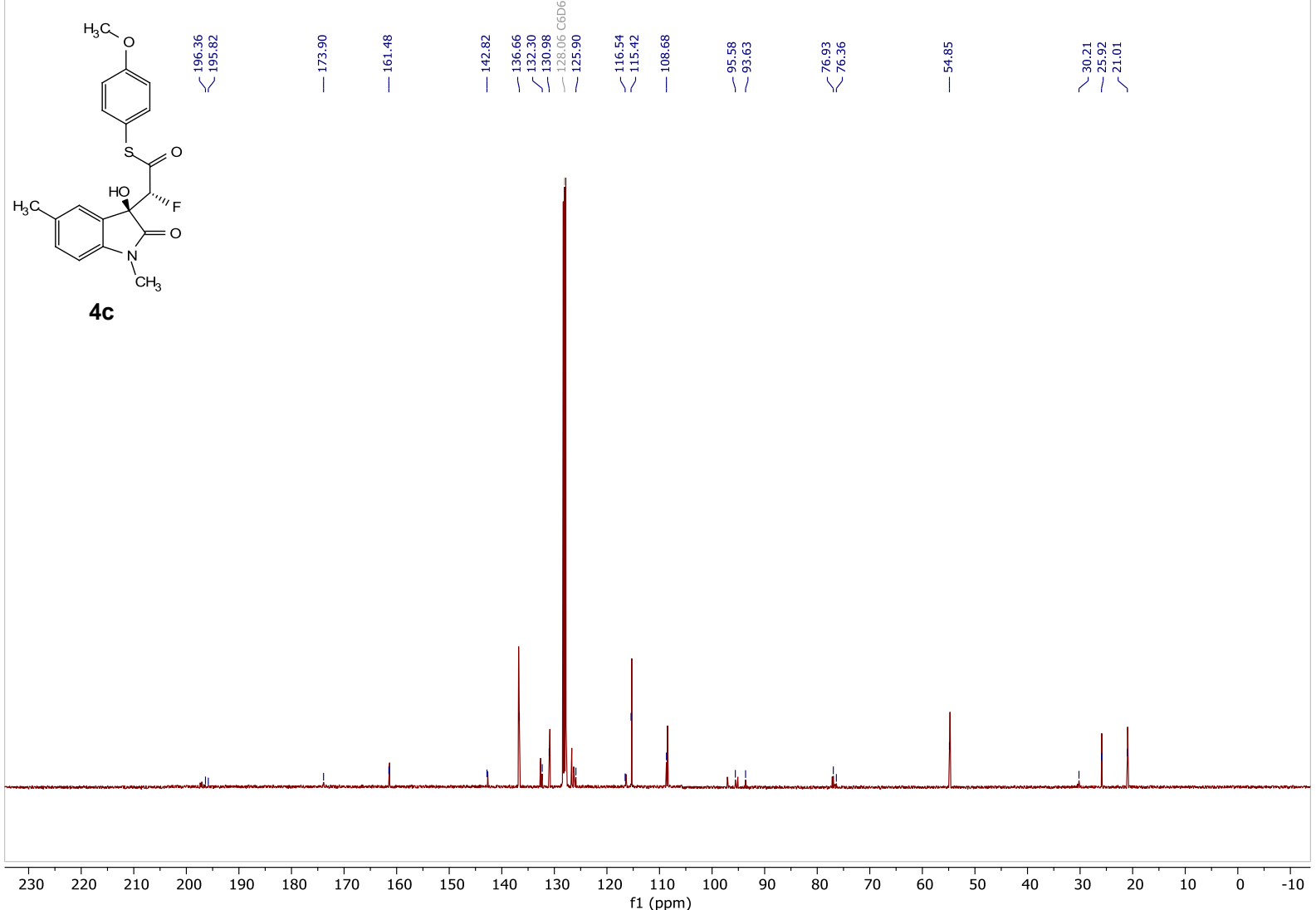


4c - ${ }^{19} \mathrm{~F}$ NMR $\left(376 \mathrm{MHz}, \mathrm{C}_{6} \mathrm{D}_{6}\right)$

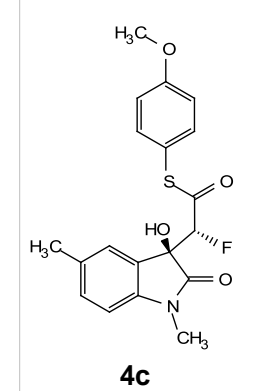

국

\%彳ำ

4c

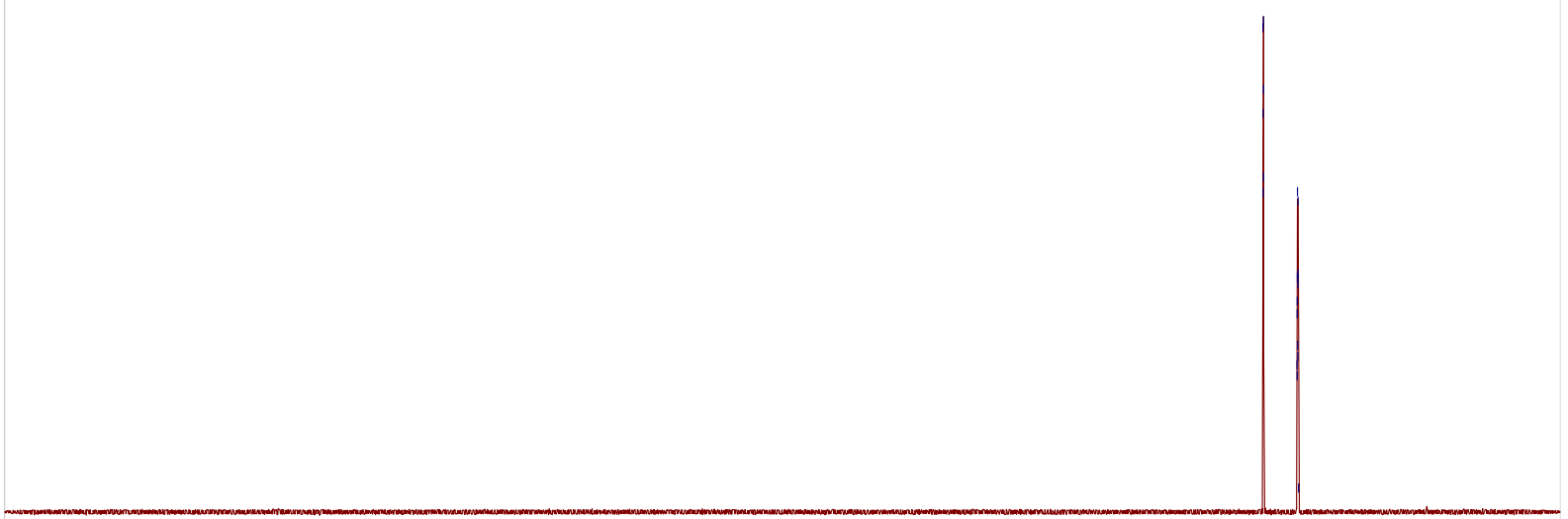

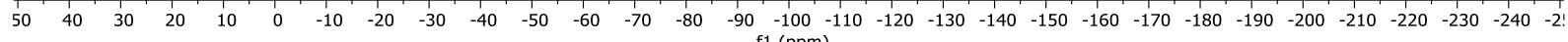

4d - ${ }^{1} \mathbf{H}$ NMR $\left(400 \mathrm{MHz}, \mathrm{C}_{6} \mathrm{D}_{6}\right)$
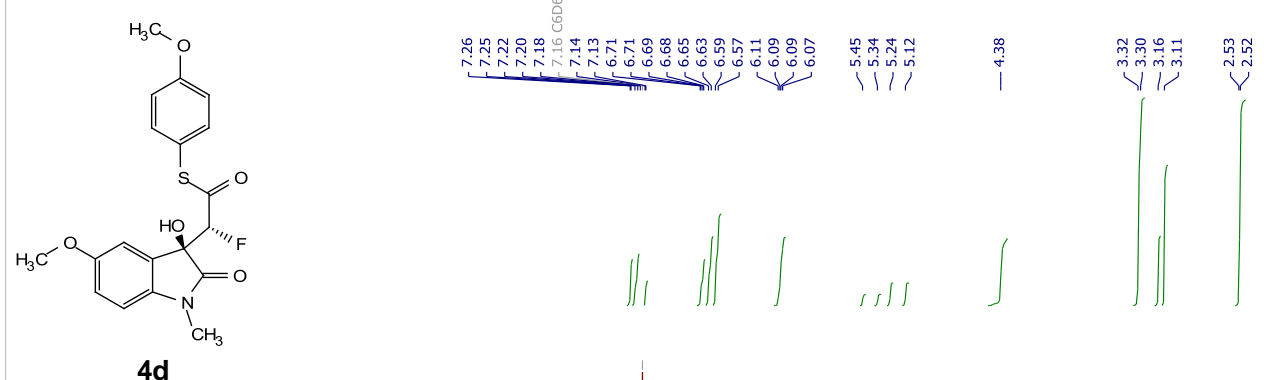

$4 d$

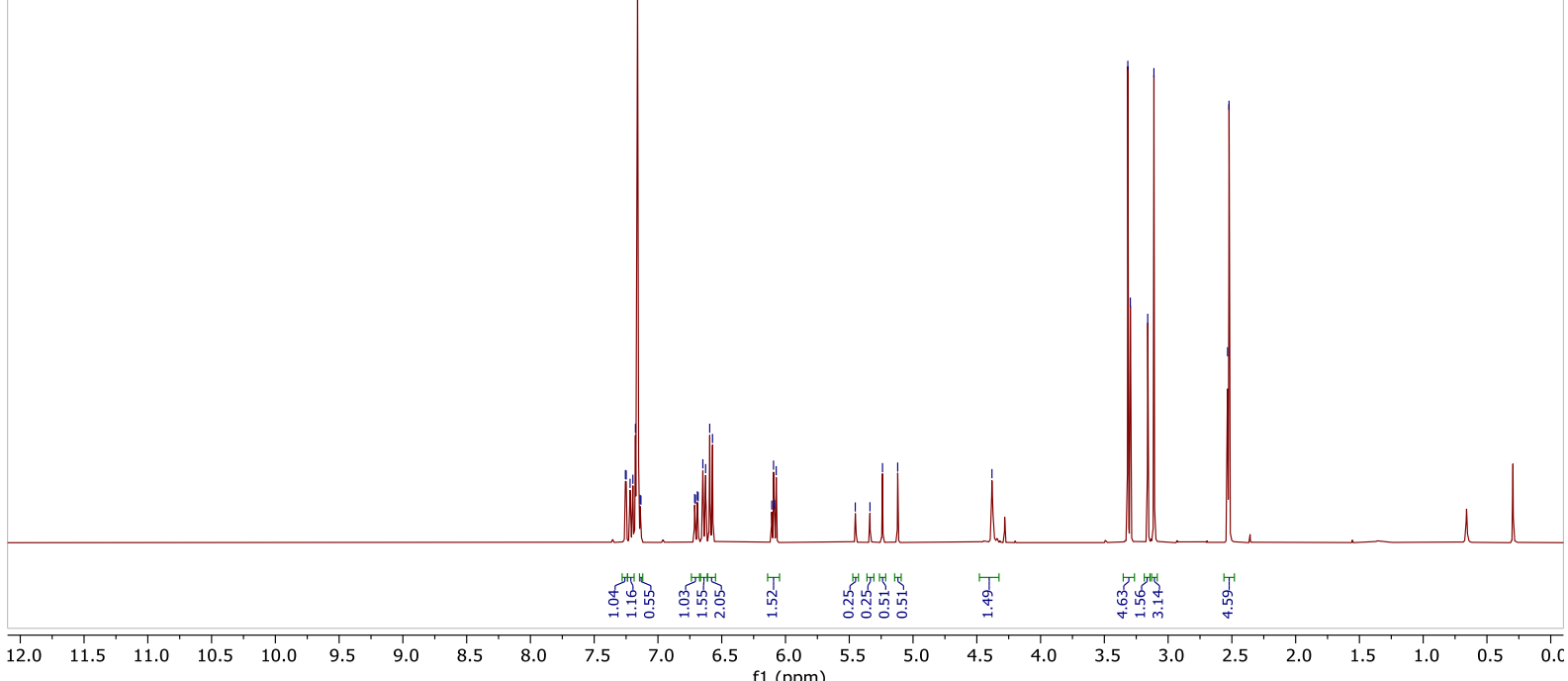


4d - ${ }^{13} \mathbf{C}$ NMR $\left(101 \mathrm{MHz}, \mathrm{C}_{6} \mathrm{D}_{6}\right)$

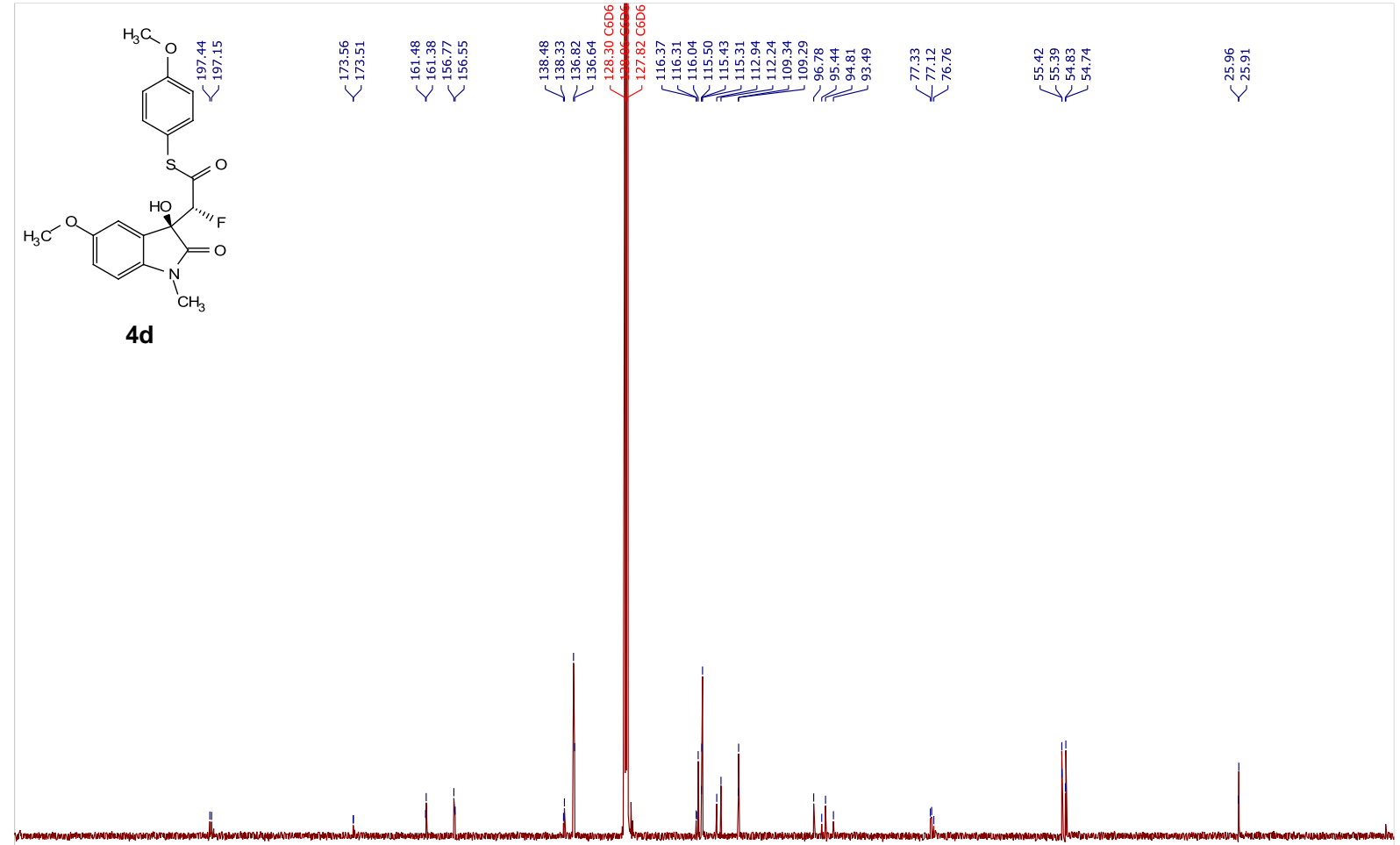

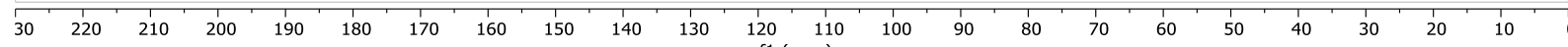

4d - ${ }^{19}$ F NMR (376 MHz, $\left.\mathrm{C}_{6} \mathrm{D}_{6}\right)$

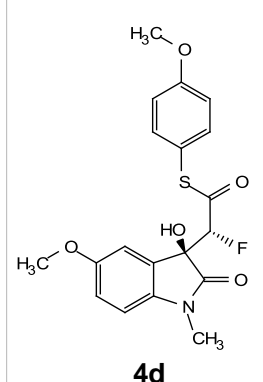

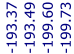

$\checkmark v$

$4 d$

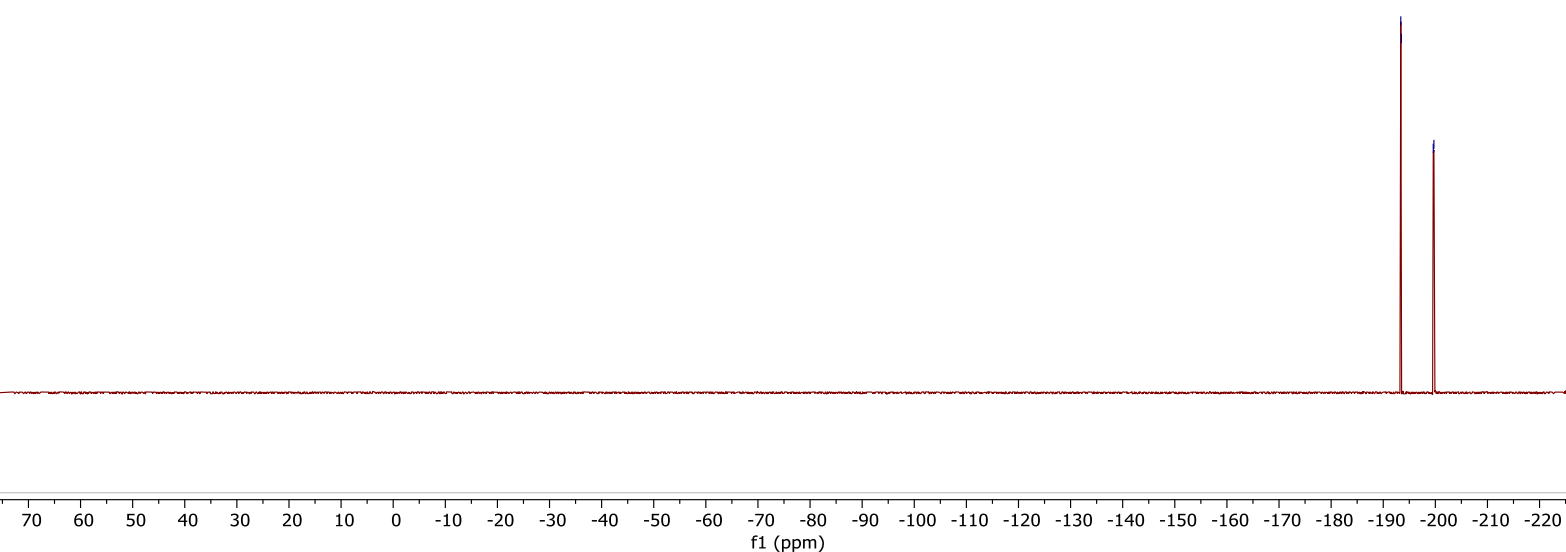


4e - ${ }^{1} \mathbf{H}$ NMR $\left(400 \mathrm{MHz}, \mathrm{CD}_{2} \mathrm{Cl}_{2}\right)$

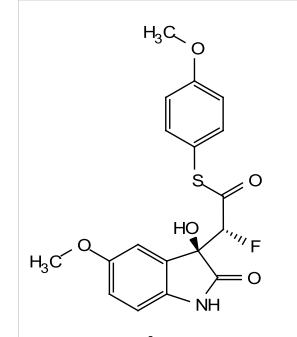

$4 e$
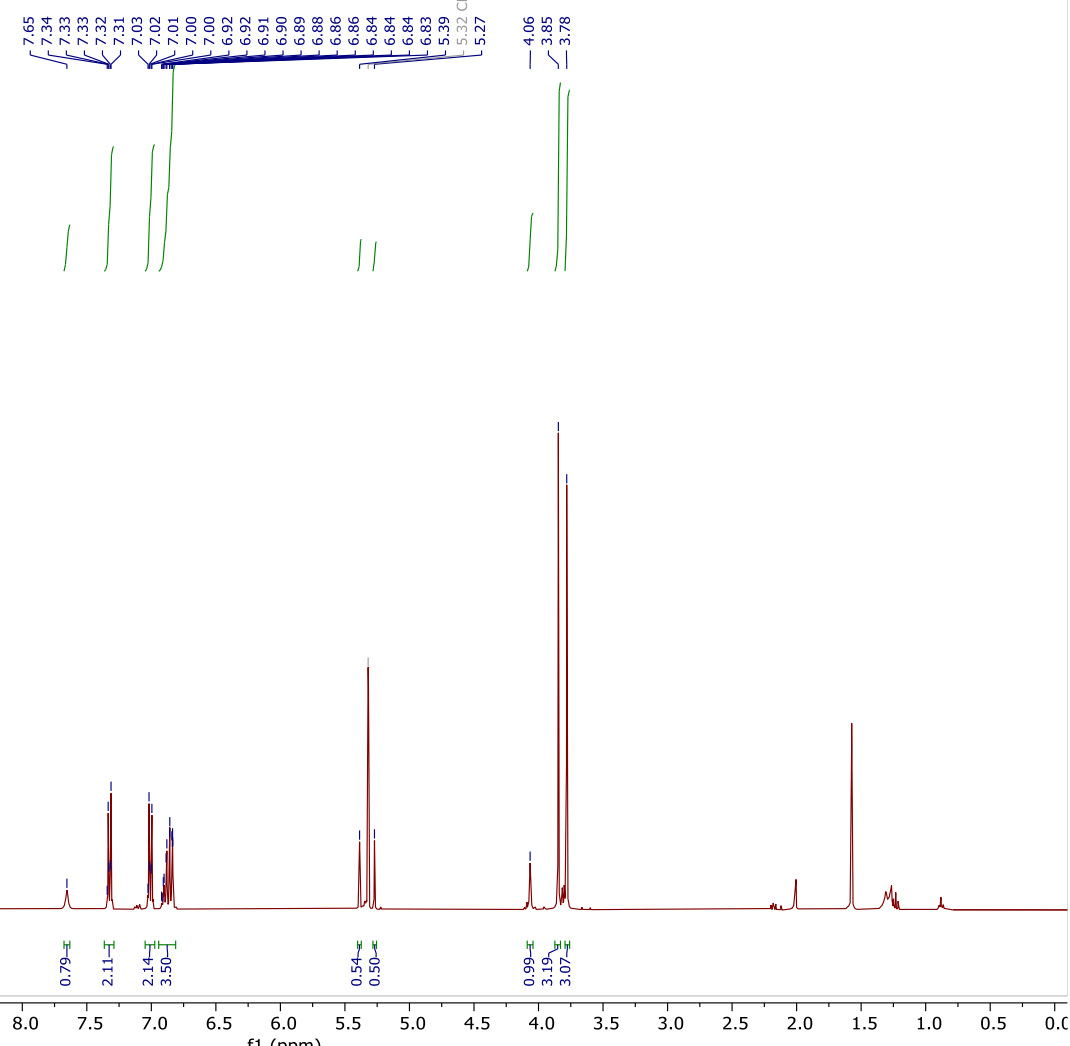

4e - ${ }^{13} \mathbf{C}$ NMR $\left(101 \mathrm{MHz}, \mathrm{CD}_{2} \mathrm{Cl}_{2}\right)$
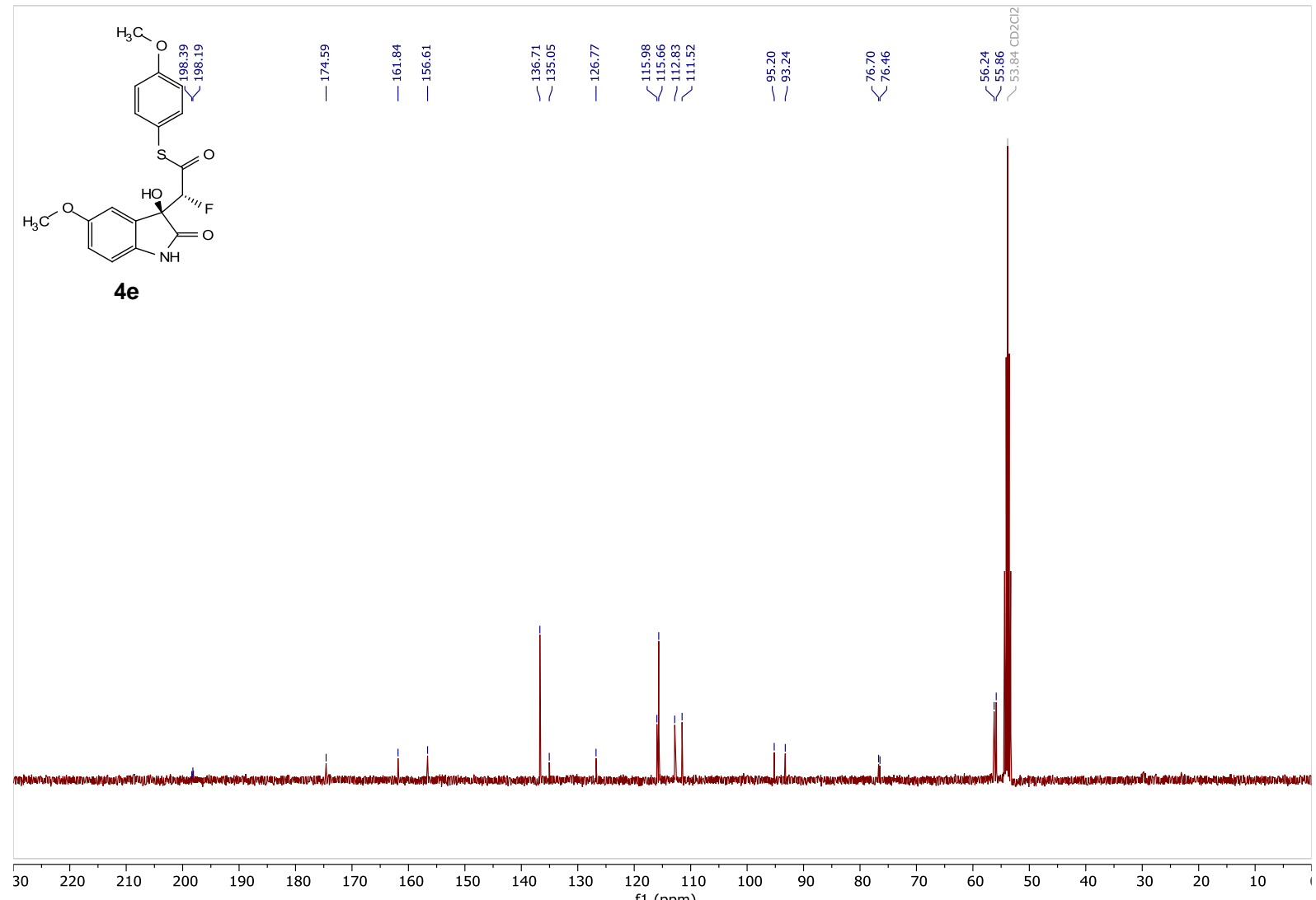
4e - ${ }^{19}$ F NMR (376 MHz, $\mathrm{CD}_{2} \mathrm{Cl}_{2}$ )

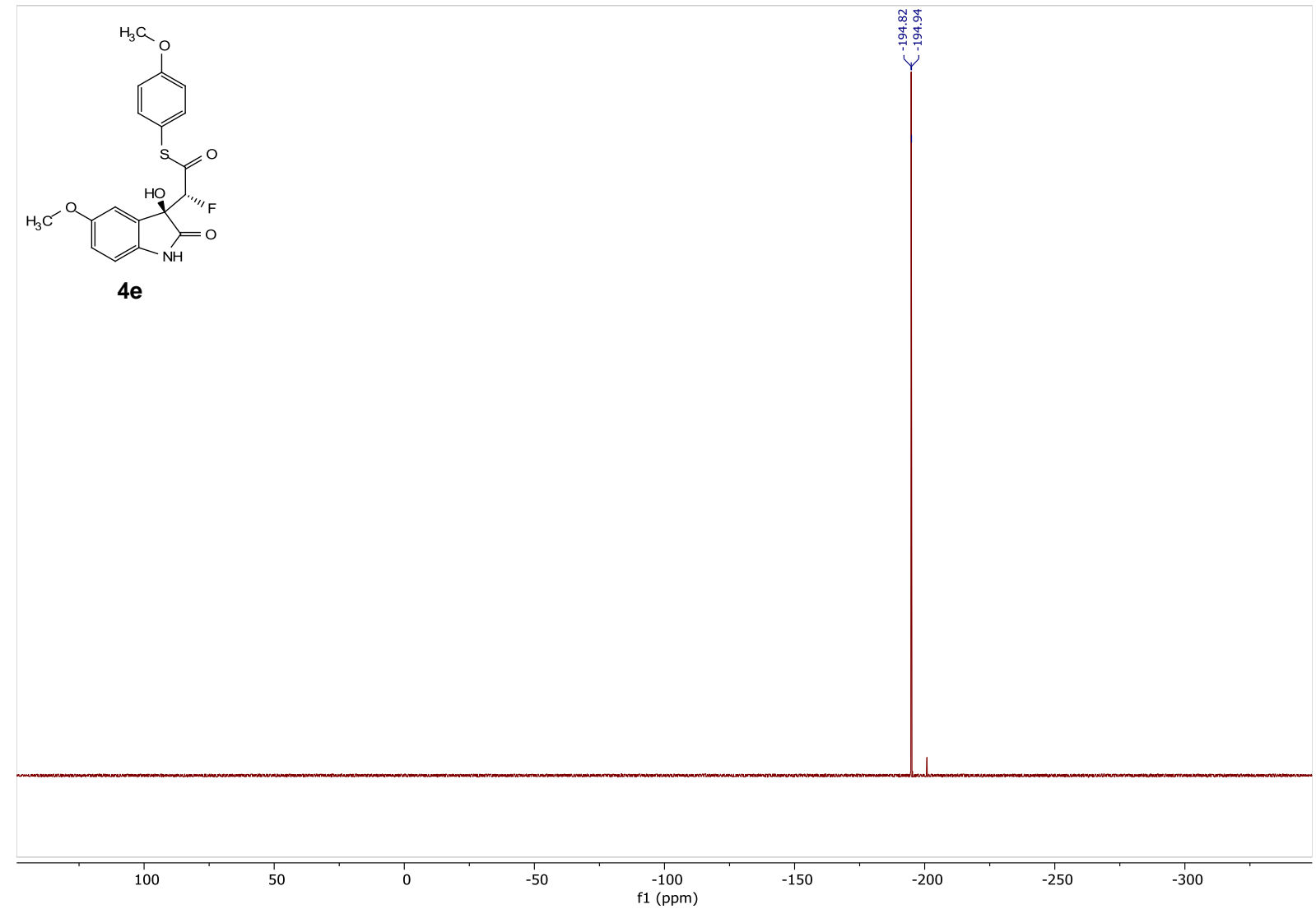

4f - ${ }^{1} \mathbf{H}$ NMR (400 MHz, $\left.\mathrm{C}_{6} \mathrm{D}_{6}\right)$

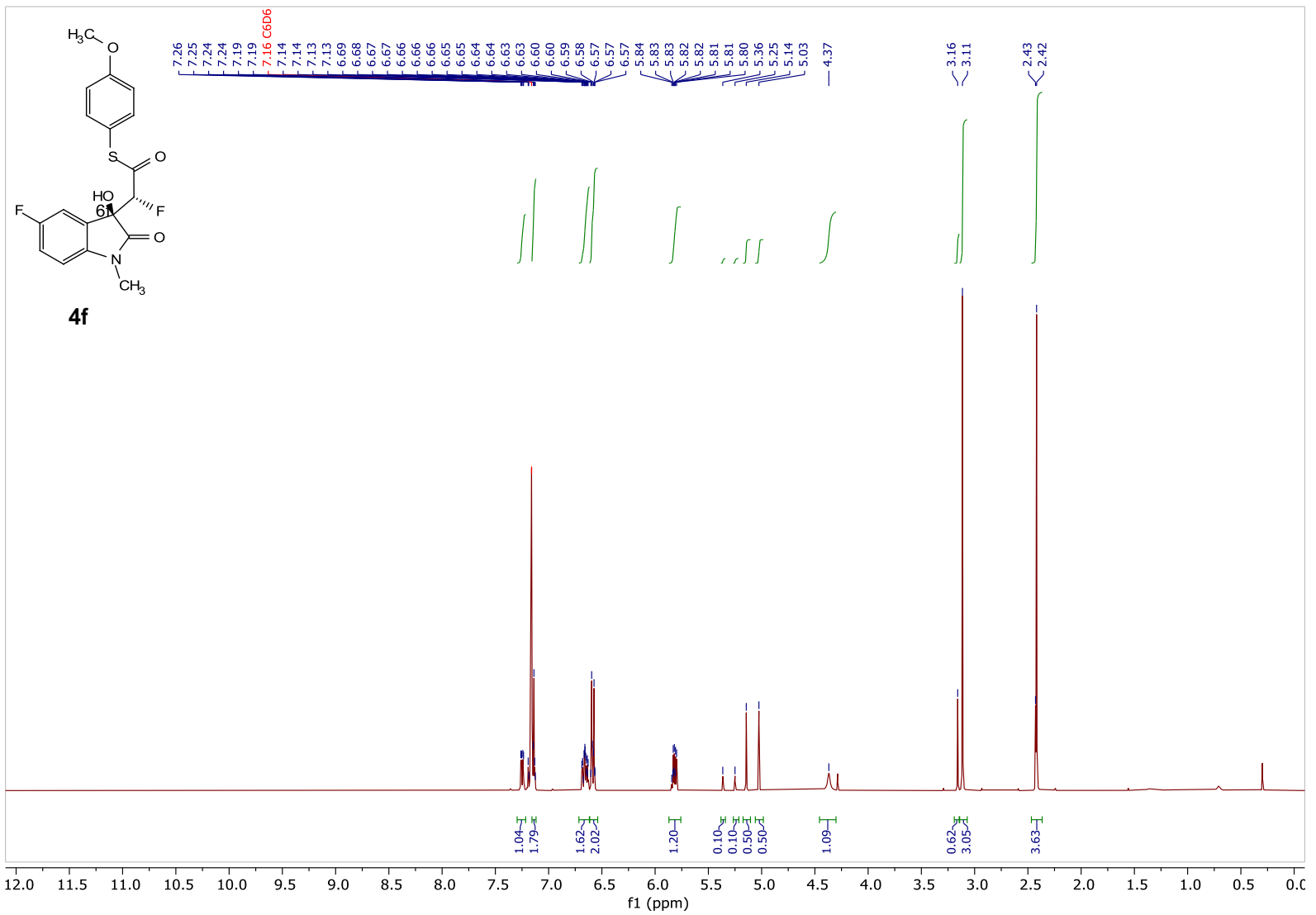


4f - ${ }^{13} \mathbf{C}$ NMR $\left(101 \mathrm{MHz}, \mathrm{C}_{6} \mathrm{D}_{6}\right)$

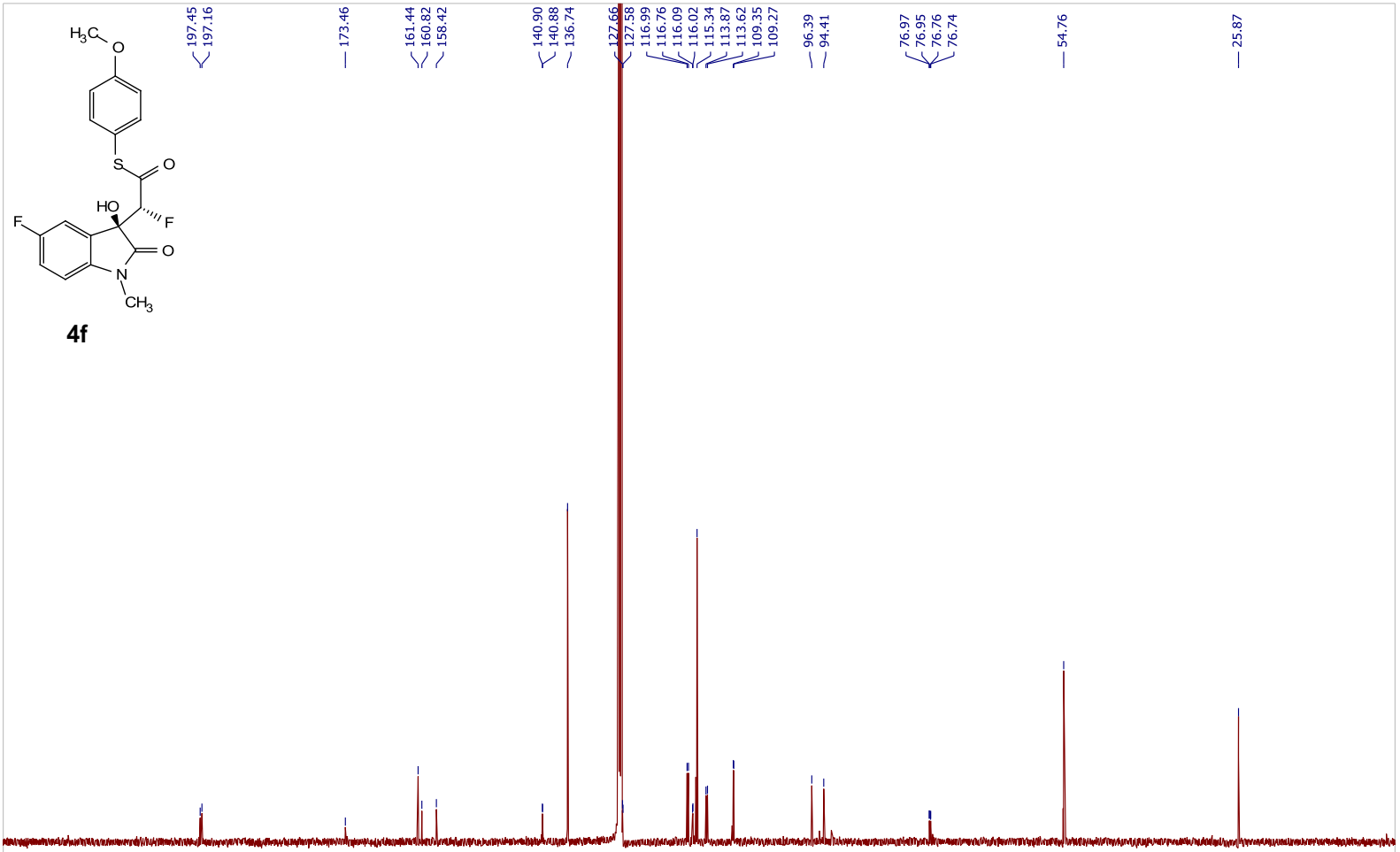

\begin{tabular}{lllllllllllllllllllllllllll}
\hline 30 & 220 & 210 & 200 & 190 & 180 & 170 & 160 & 150 & 140 & 130 & 120 & 110 & 100 & 90 & 80 & 70 & 60 & 50 & 40 & 30 & 20 & 10 & 10
\end{tabular}

\section{4f - ${ }^{19} \mathbf{F}$ NMR $\left(376 \mathrm{MHz}, \mathrm{C}_{6} \mathrm{D}_{6}\right)$}

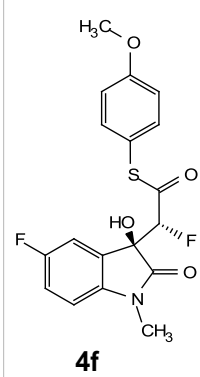

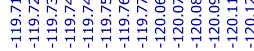

Vir

Ү

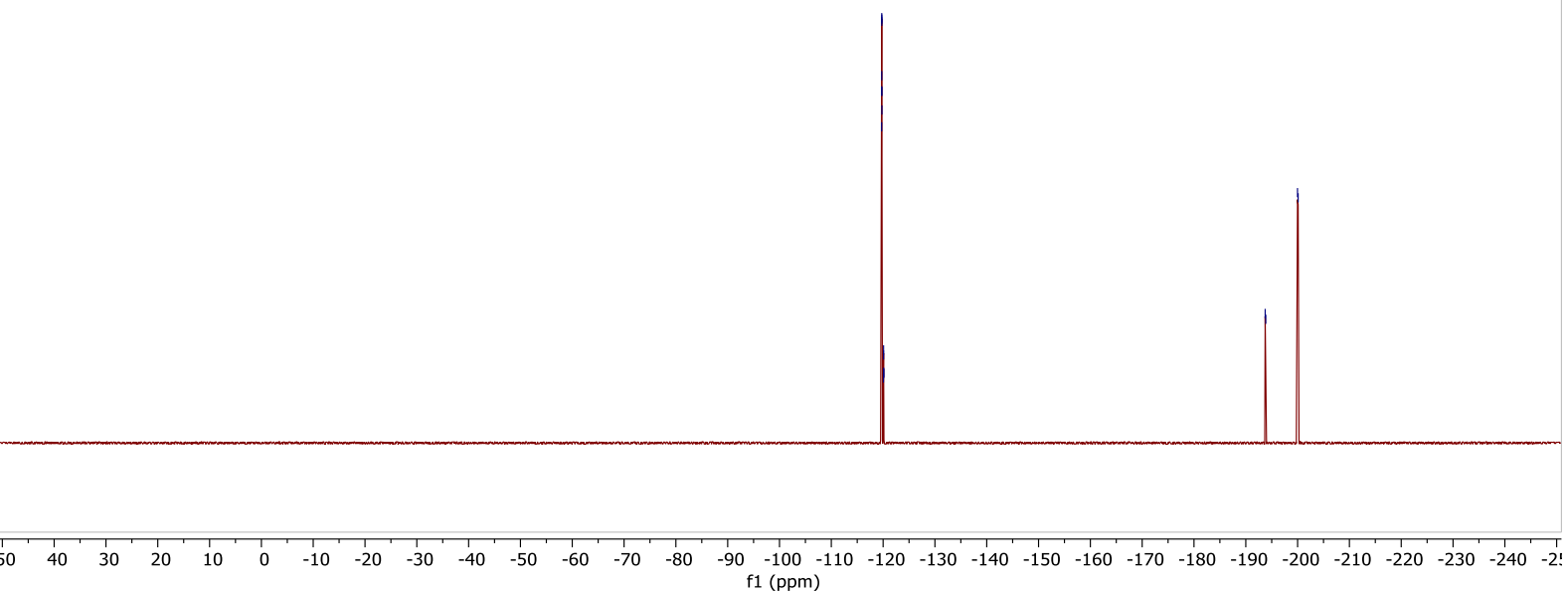


4g - ${ }^{1} \mathbf{H}$ NMR $\left(400 \mathrm{MHz}, \mathrm{C}_{6} \mathrm{D}_{6}\right)$

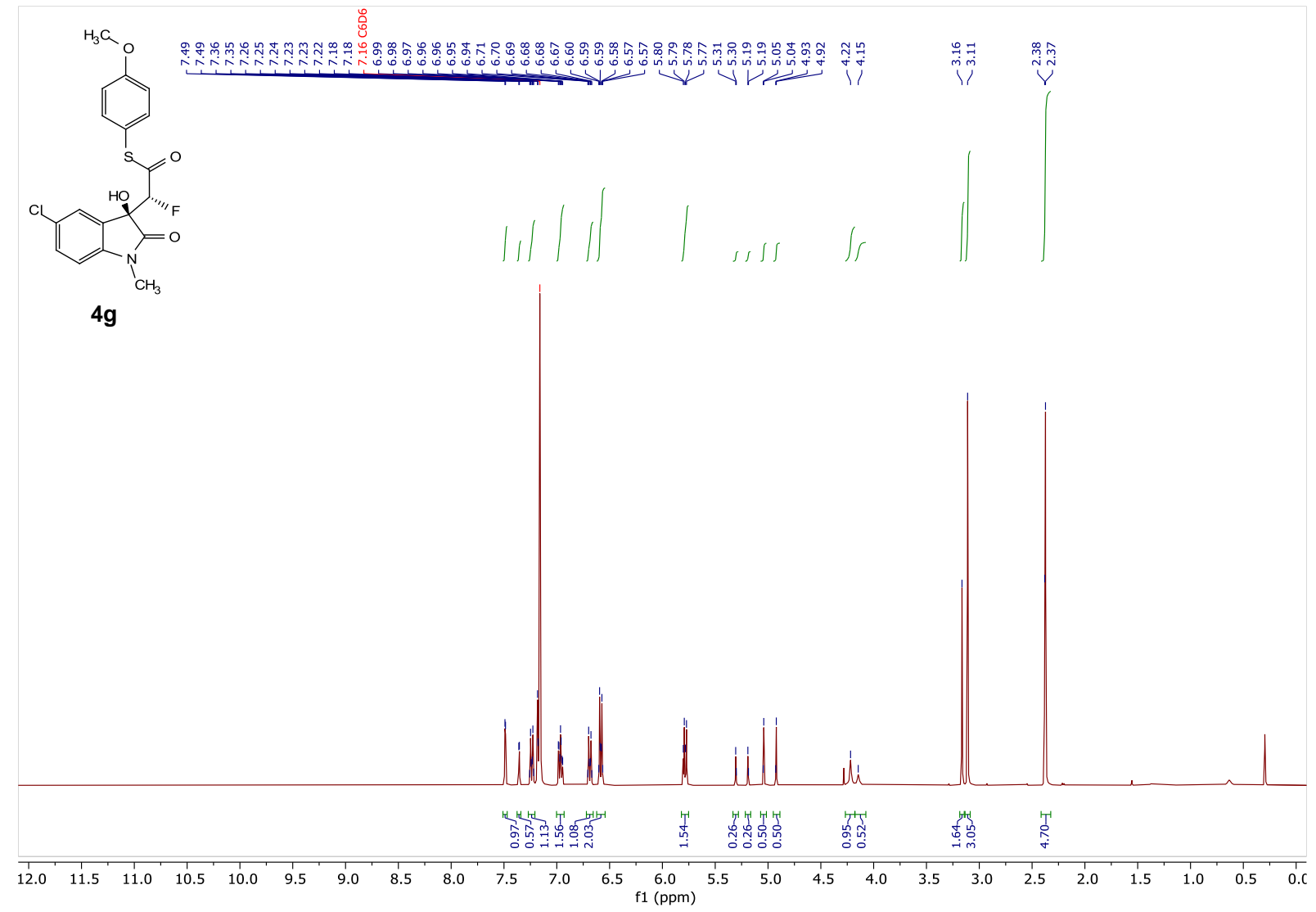

4g - ${ }^{13} \mathbf{C}$ NMR $\left(101 \mathrm{MHz}, \mathrm{C}_{6} \mathrm{D}_{6}\right)$

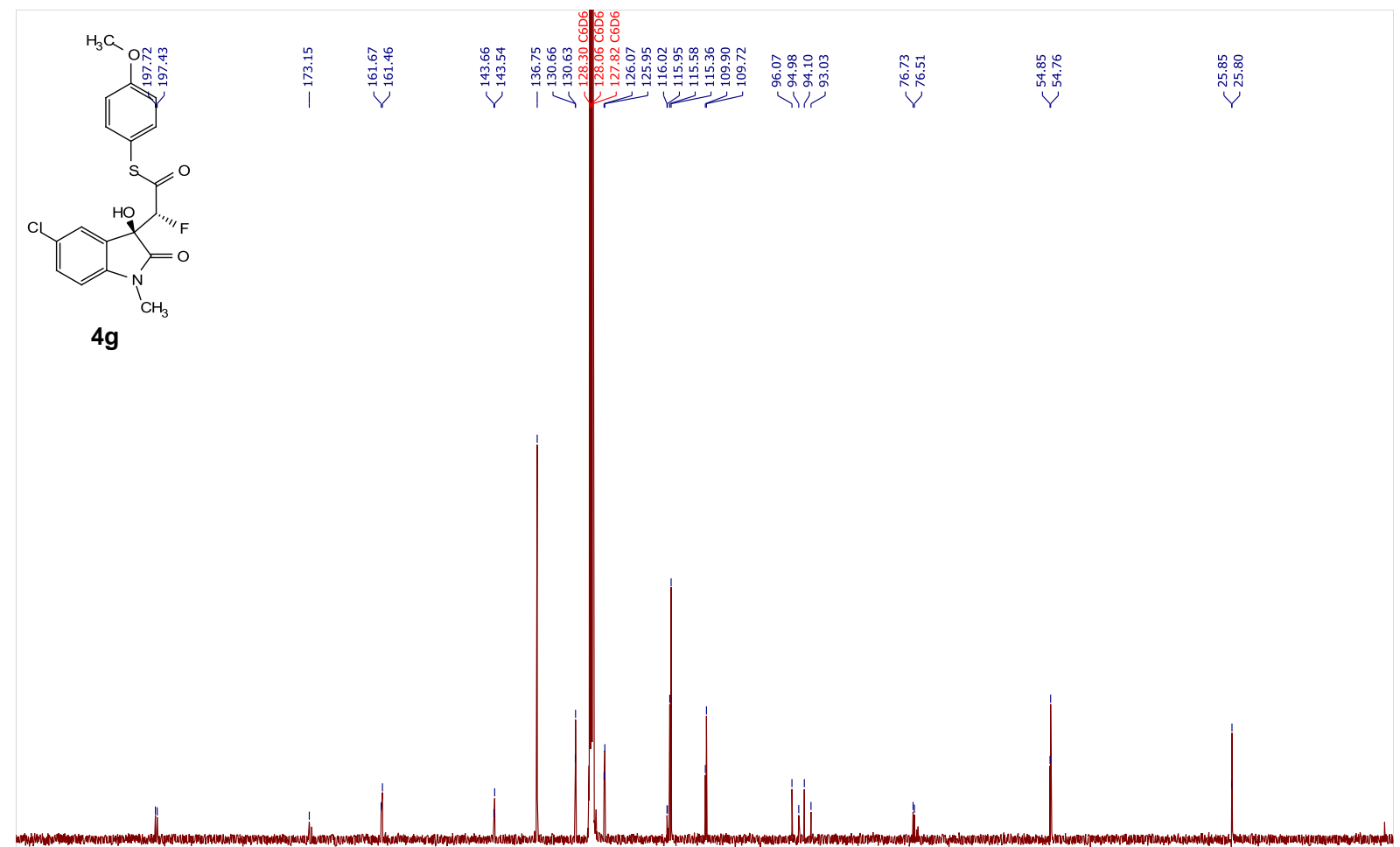

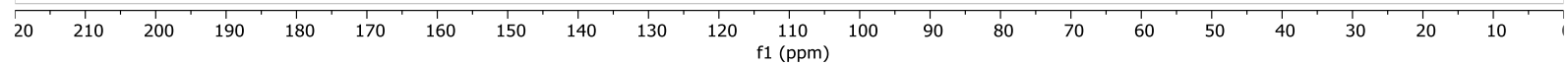


4g - ${ }^{19}$ F NMR (376 MHz, $\left.\mathrm{C}_{6} \mathrm{D}_{6}\right)$
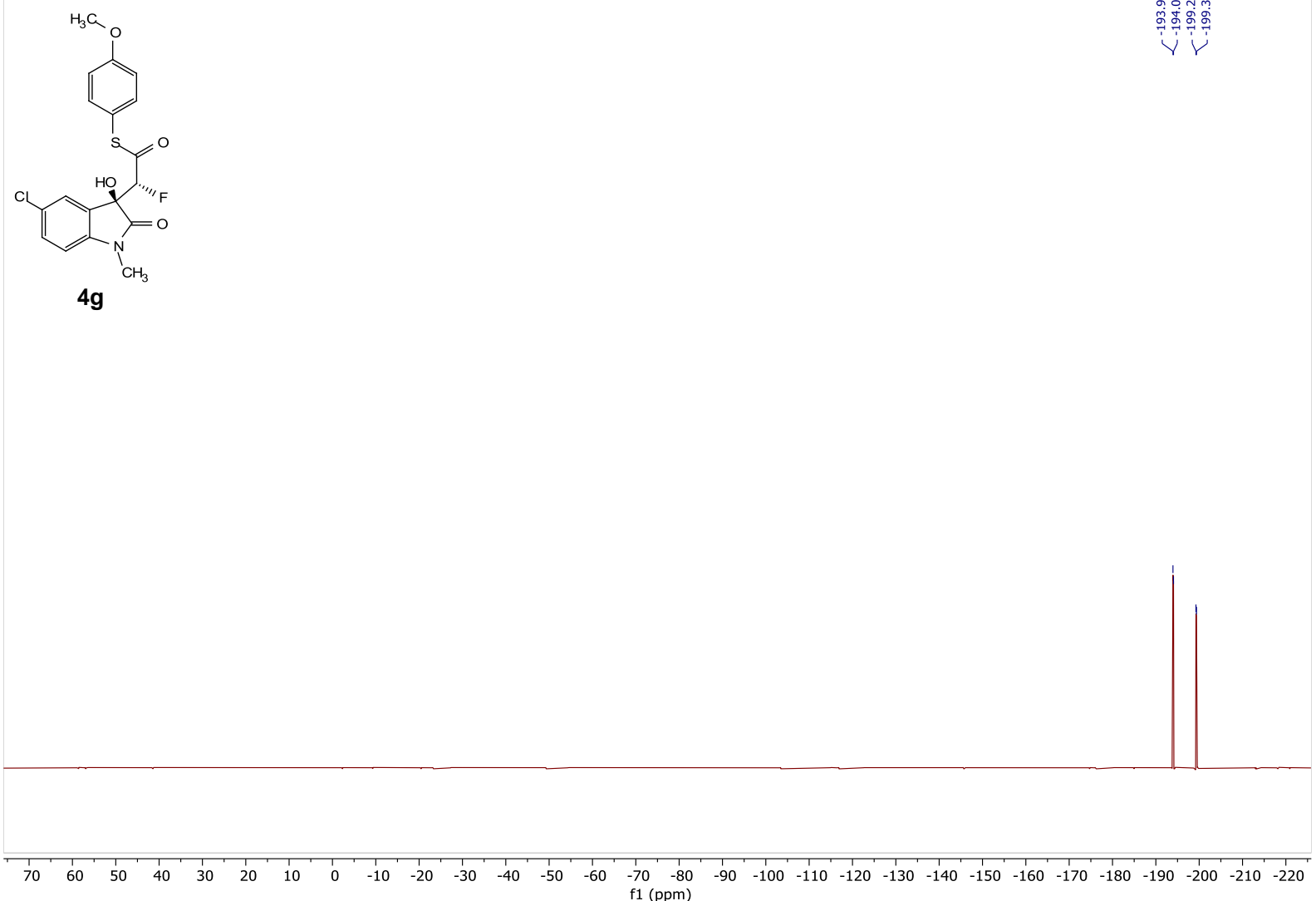

4h - ${ }^{1} \mathrm{H}$ NMR $\left(400 \mathrm{MHz}, \mathrm{C}_{6} \mathrm{D}_{6}\right)$

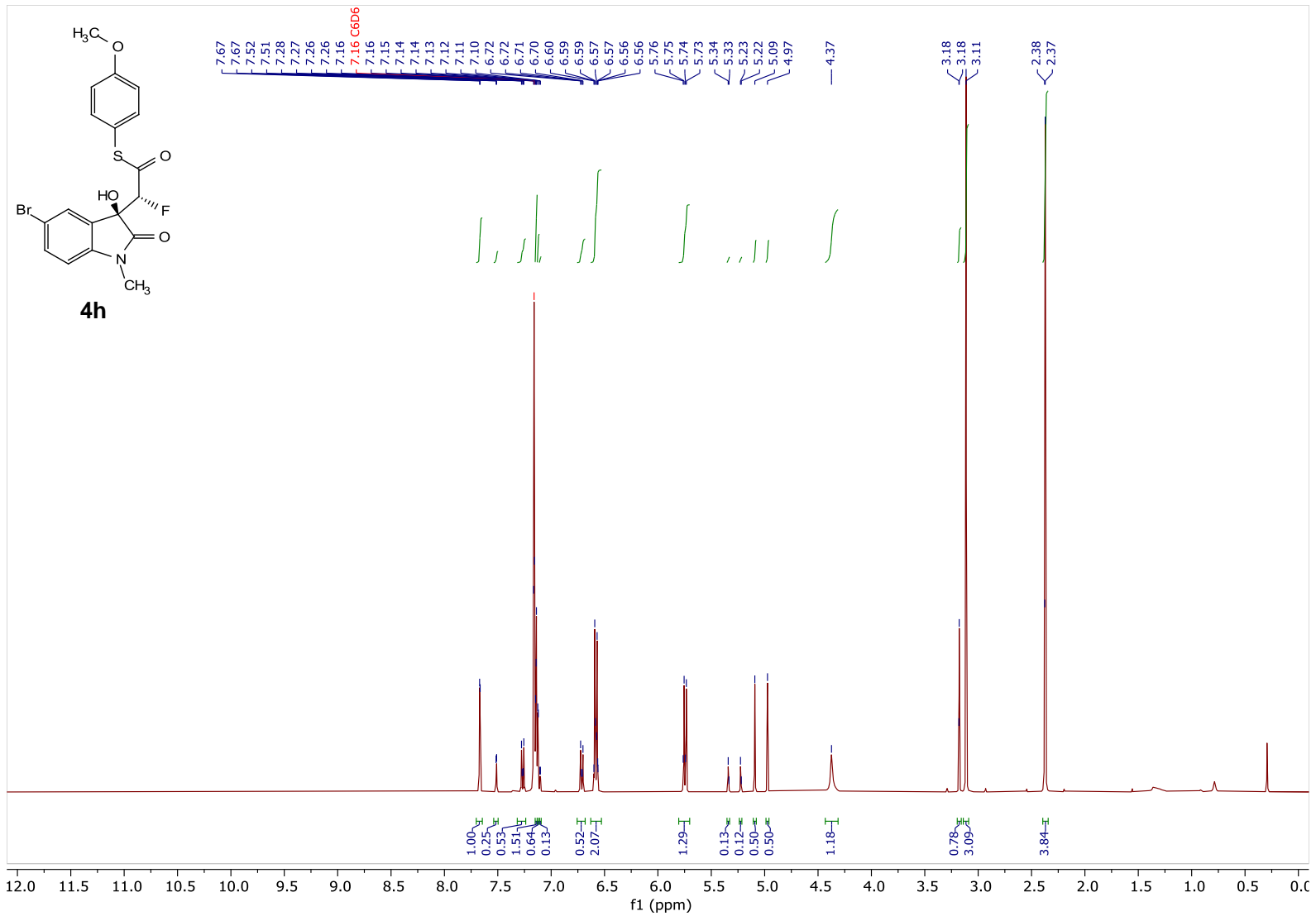


Ah - ${ }^{13}$ C NMR (101 MHz, $\left.C_{6} D_{6}\right)$

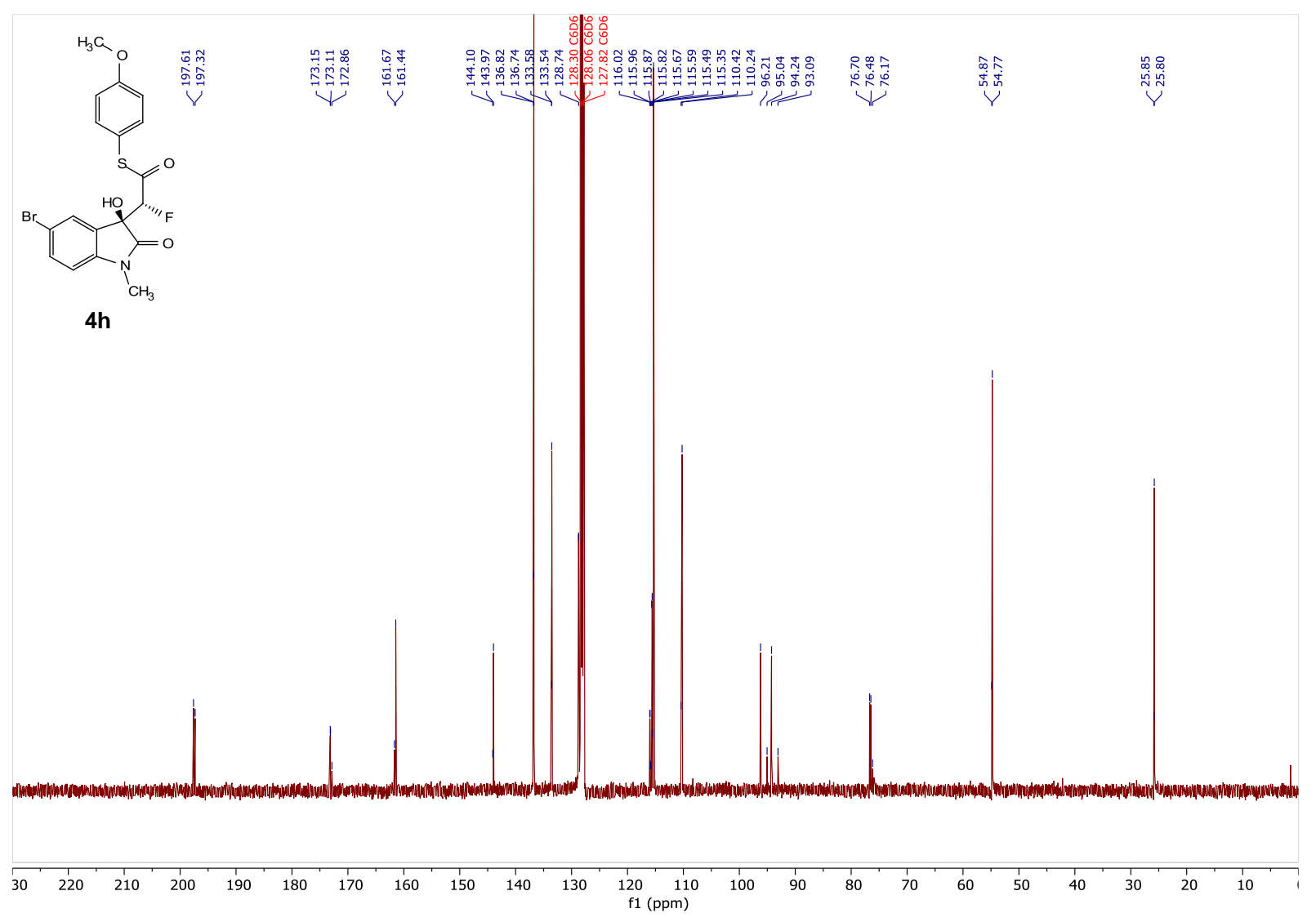

Th - ${ }^{19}$ F NMR (376 MHz, $\left.\mathrm{C}_{6} \mathrm{D}_{6}\right)$

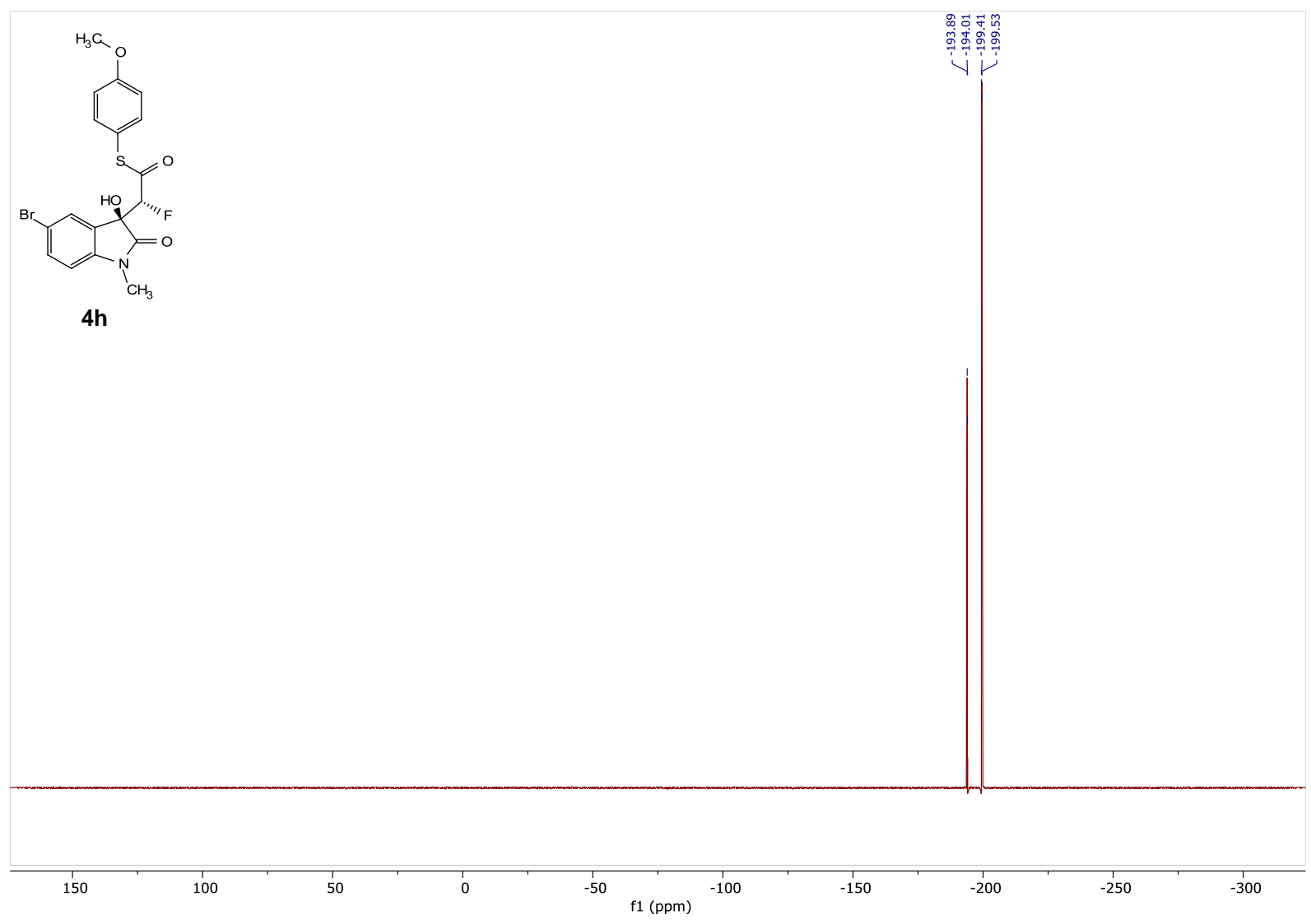




\section{4i - ${ }^{1} \mathbf{H}$ NMR (400 MHz, $\left.\mathrm{C}_{6} \mathrm{D}_{6}\right)$}

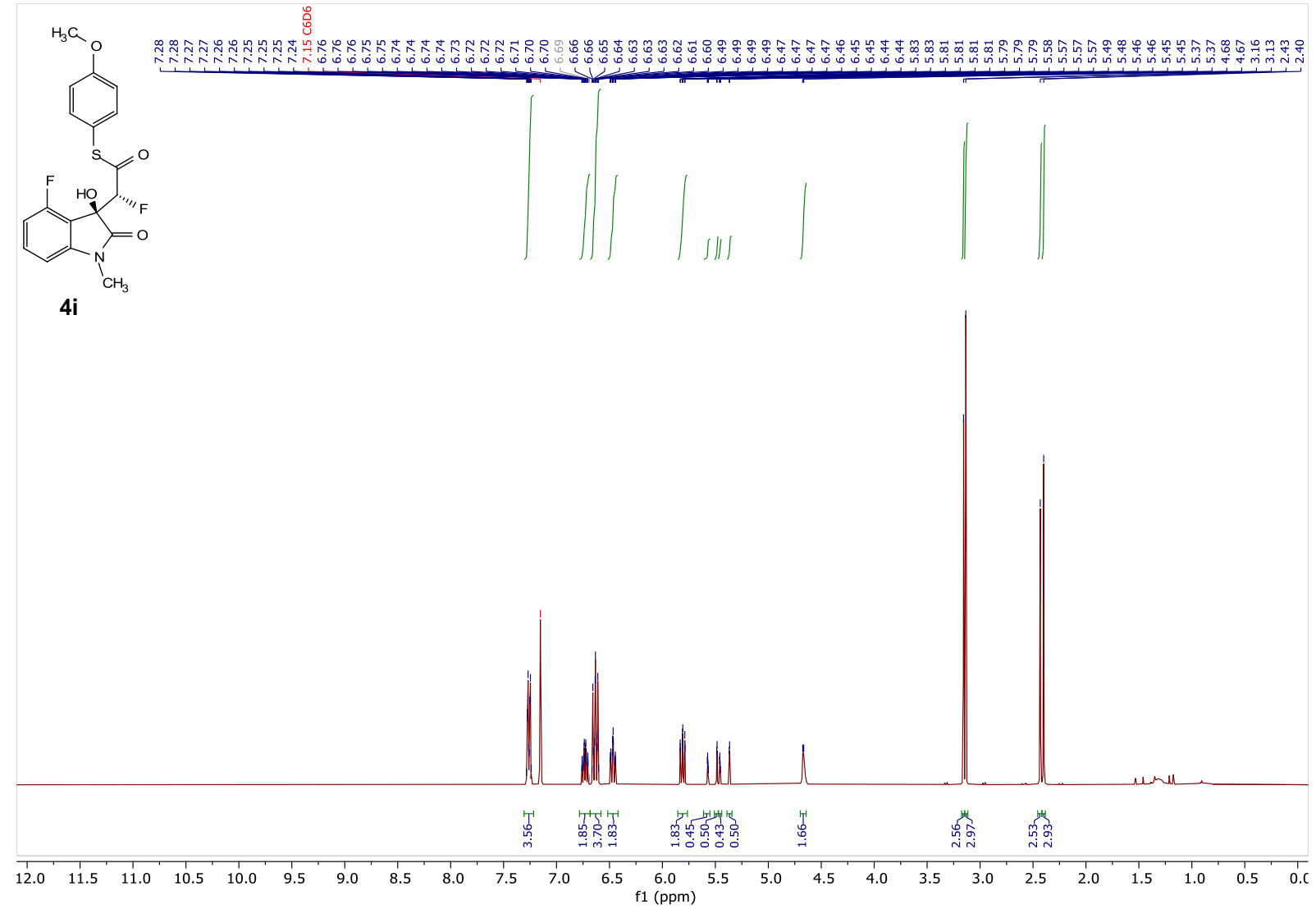

4i - ${ }^{13}$ C NMR $\left(101 \mathrm{MHz}, \mathrm{C}_{6} \mathrm{D}_{6}\right)$

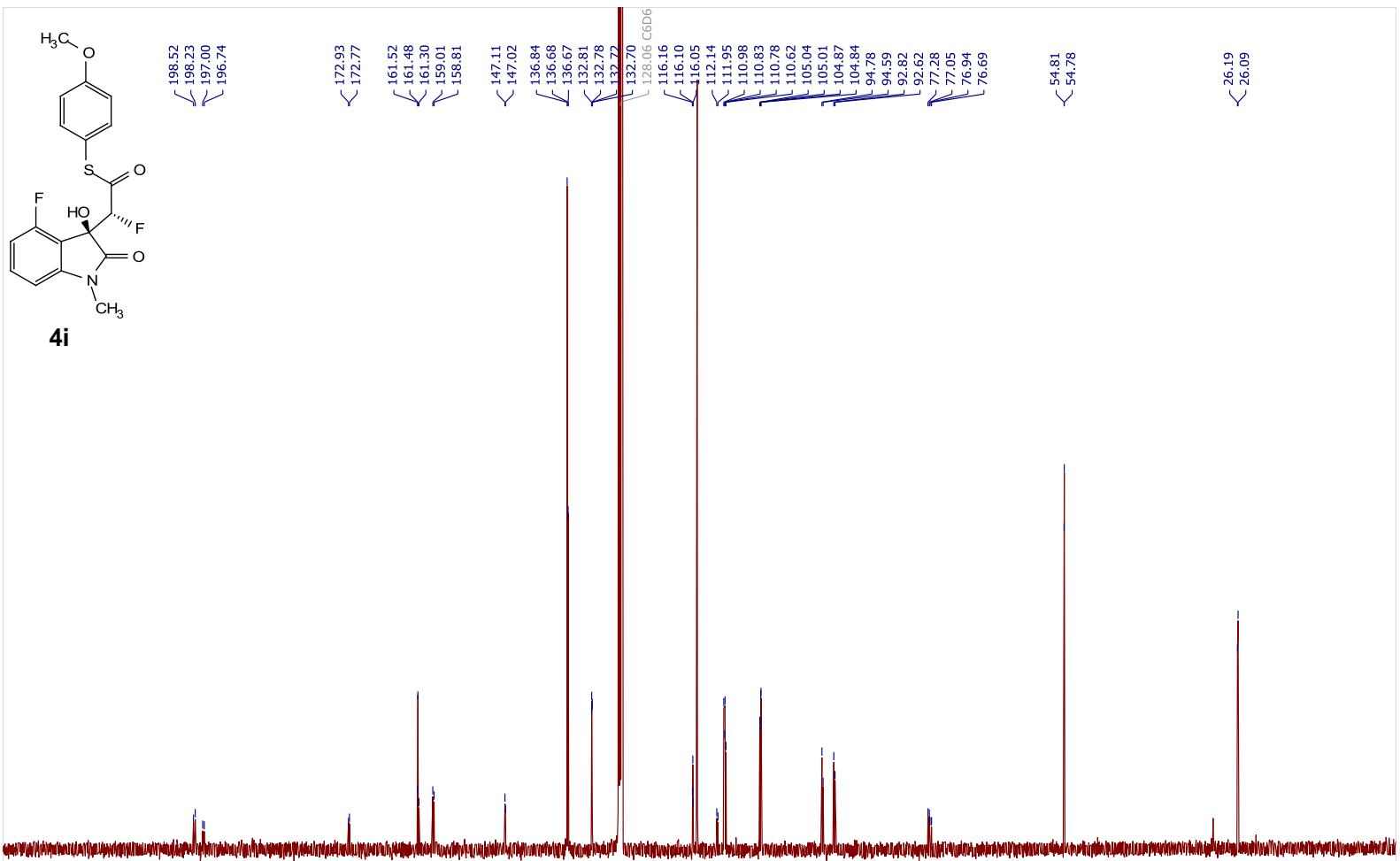

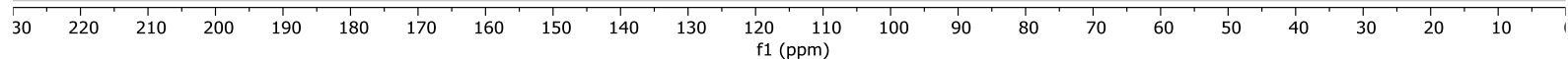


Li - ${ }^{19}$ F NMR $\left(376 \mathrm{MHz}, \mathrm{C}_{6} \mathrm{D}_{6}\right)$

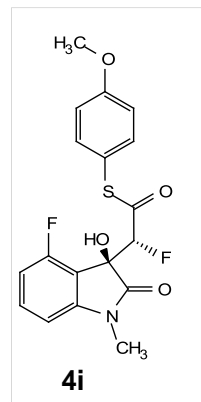

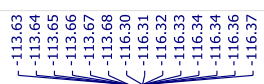

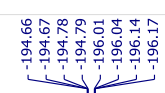

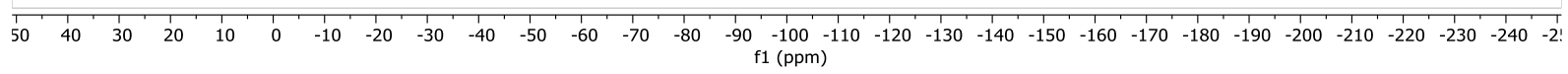

4j - ${ }^{1} \mathbf{H}$ NMR $\left(400 \mathrm{MHz}, \mathrm{CD}_{2} \mathrm{Cl}_{2}\right)$

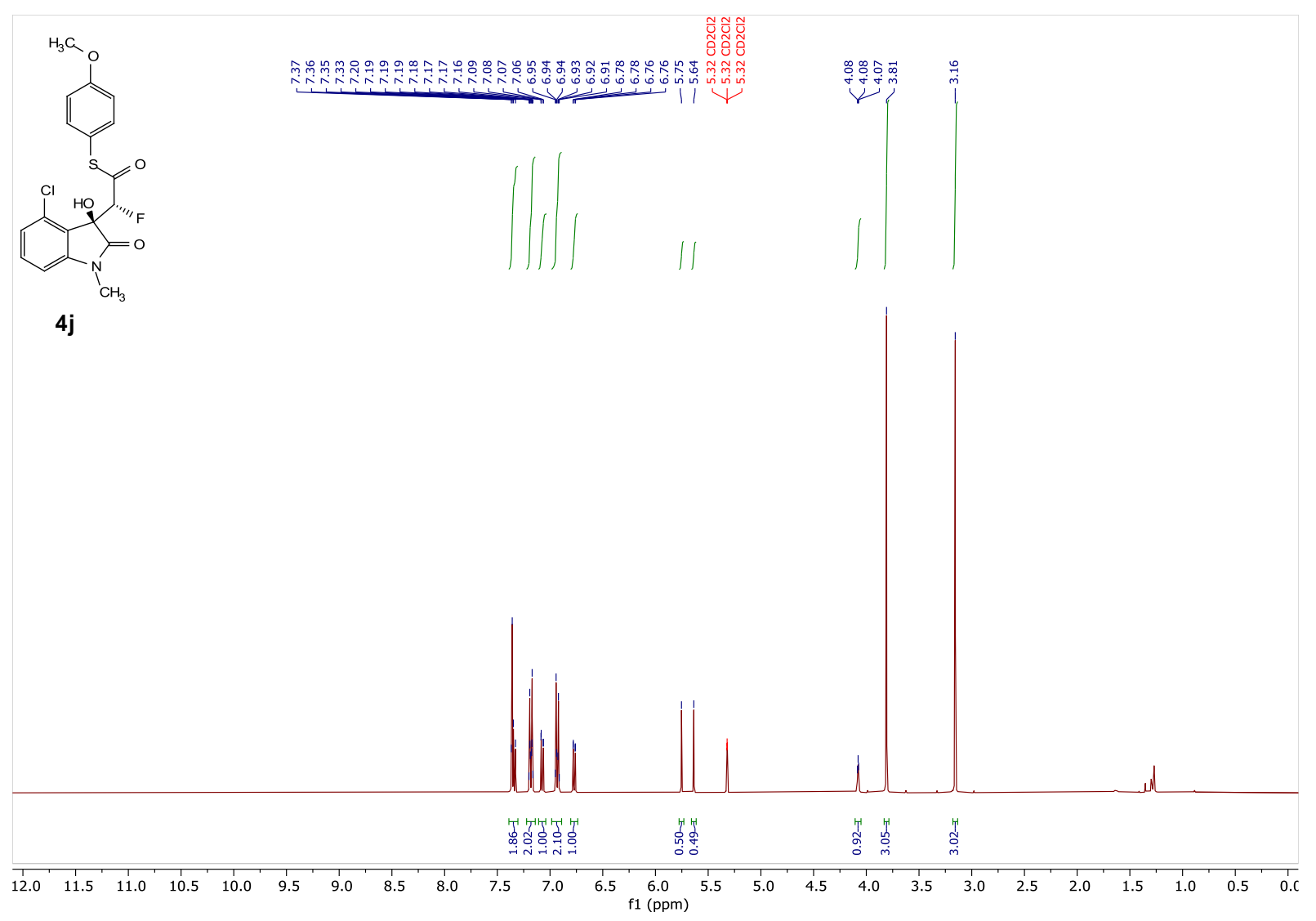


4j - ${ }^{13} \mathbf{C}$ NMR (101 MHz, $\left.\mathrm{CD}_{2} \mathrm{Cl}_{2}\right)$

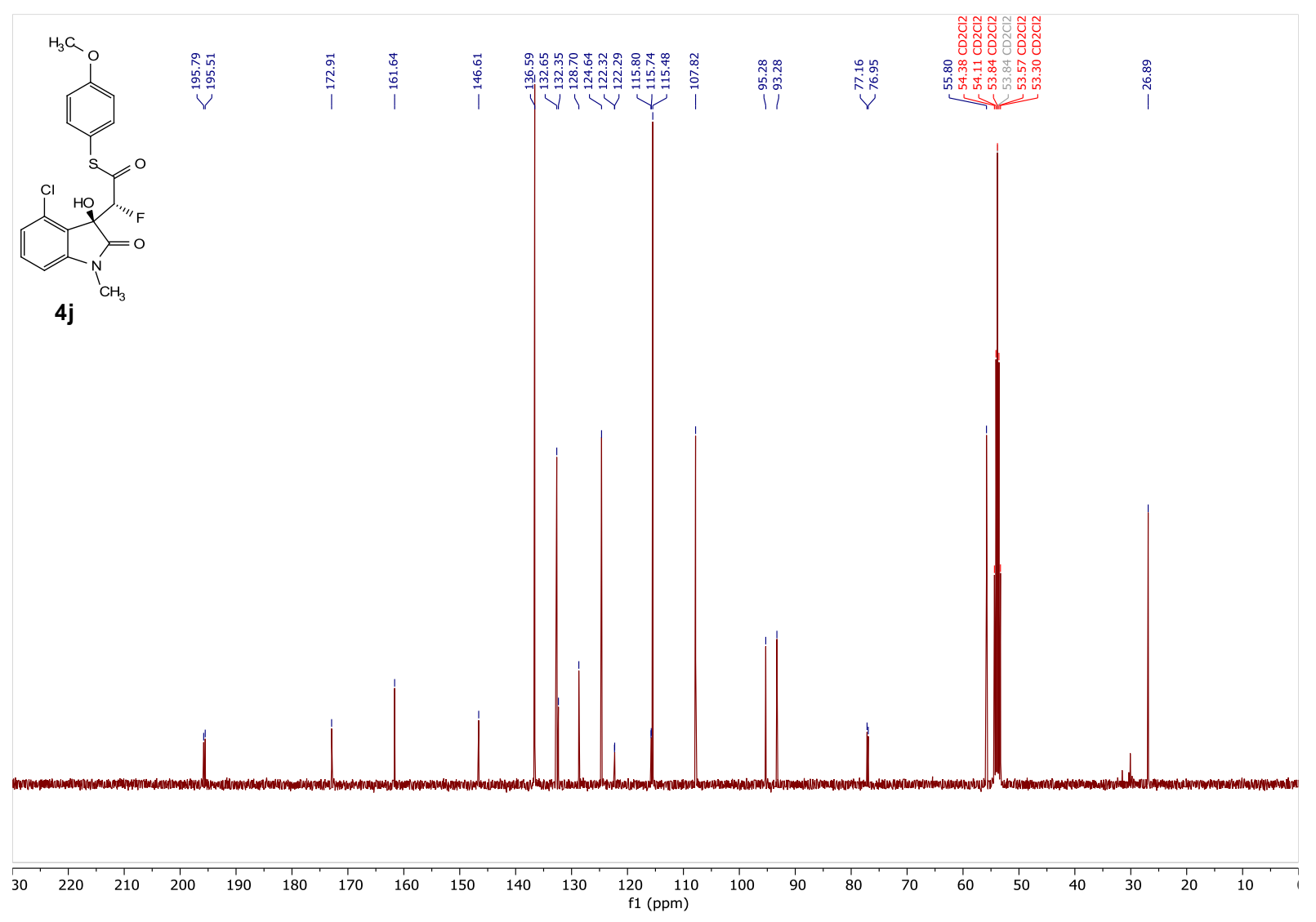

4j - ${ }^{19}$ F NMR (376 MHz, $\left.\mathrm{CD}_{2} \mathrm{Cl}_{2}\right)$

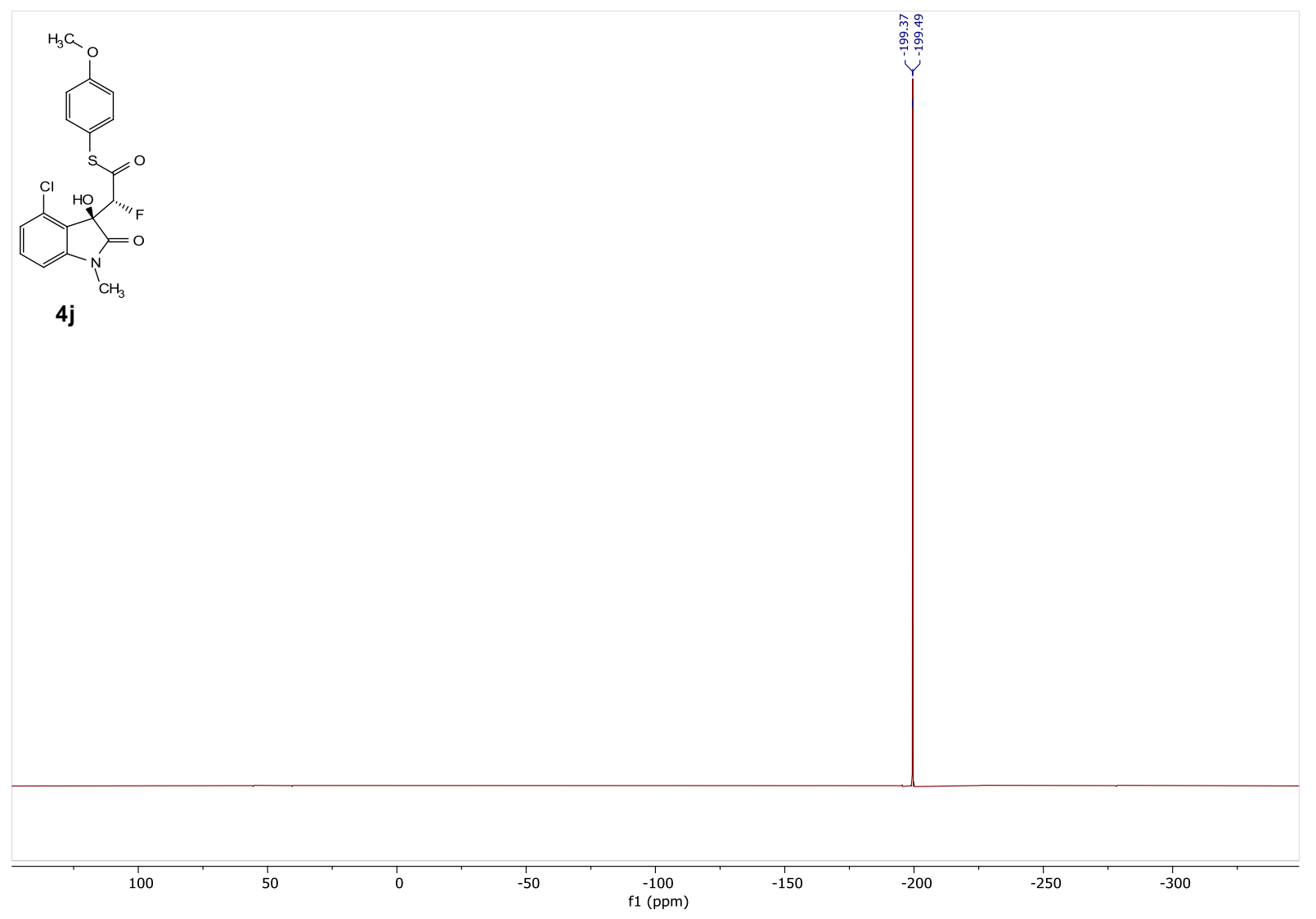


4k - ' $\mathbf{H}$ NMR (600 MHz, d6-acetone)
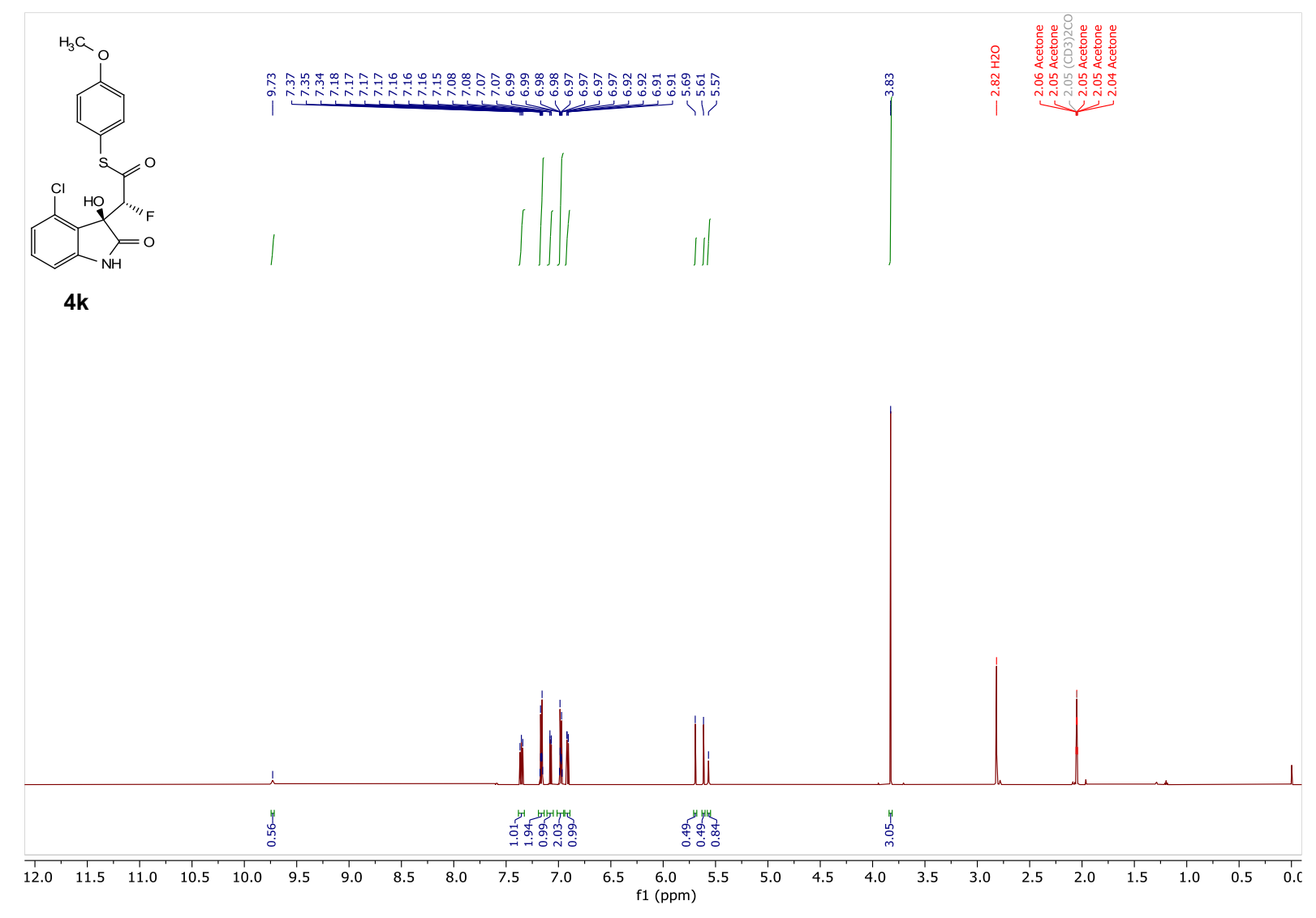

4k - ${ }^{13}$ C NMR (151 MHz, d6-acetone)

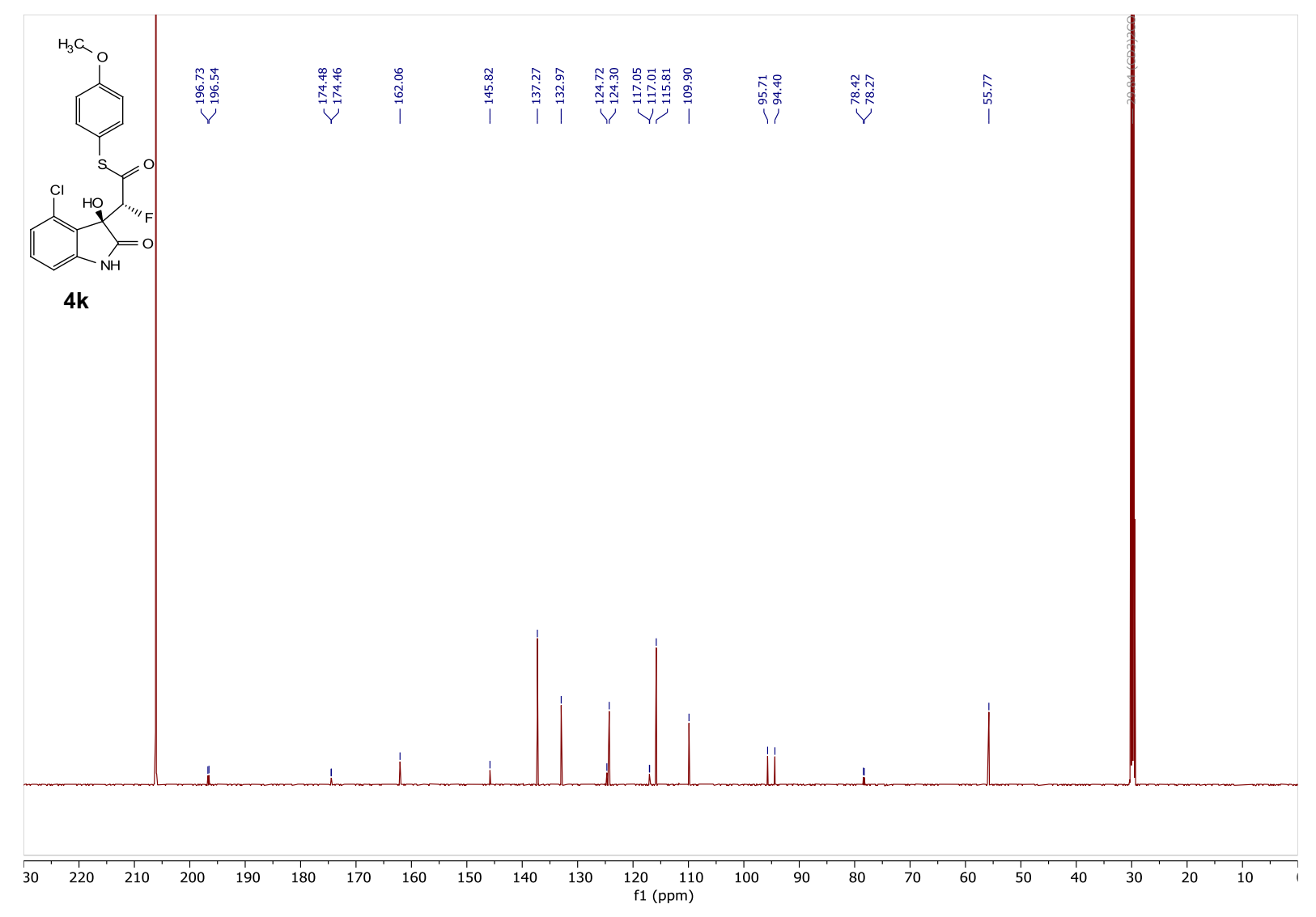


4k - ${ }^{19}$ F NMR (470 MHz, d6-acetone)

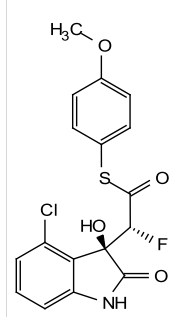

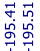

$4 k$

$\begin{array}{lll}-145 & -150 & -155\end{array}$

$-160$

$55 \quad-190$

4k - Other diastereoisomer: ${ }^{1} \mathbf{H}$ NMR (600 MHz, d6-acetone)

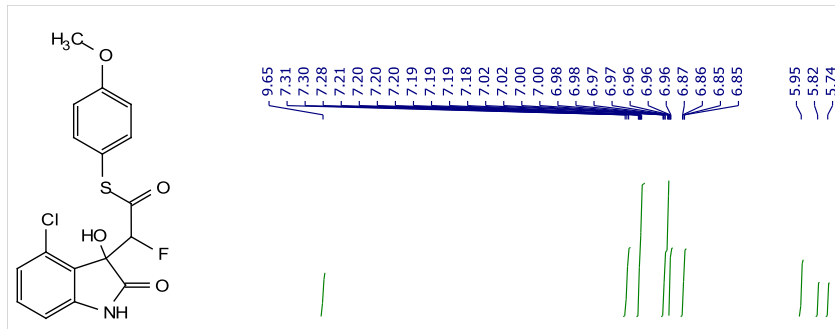

4k
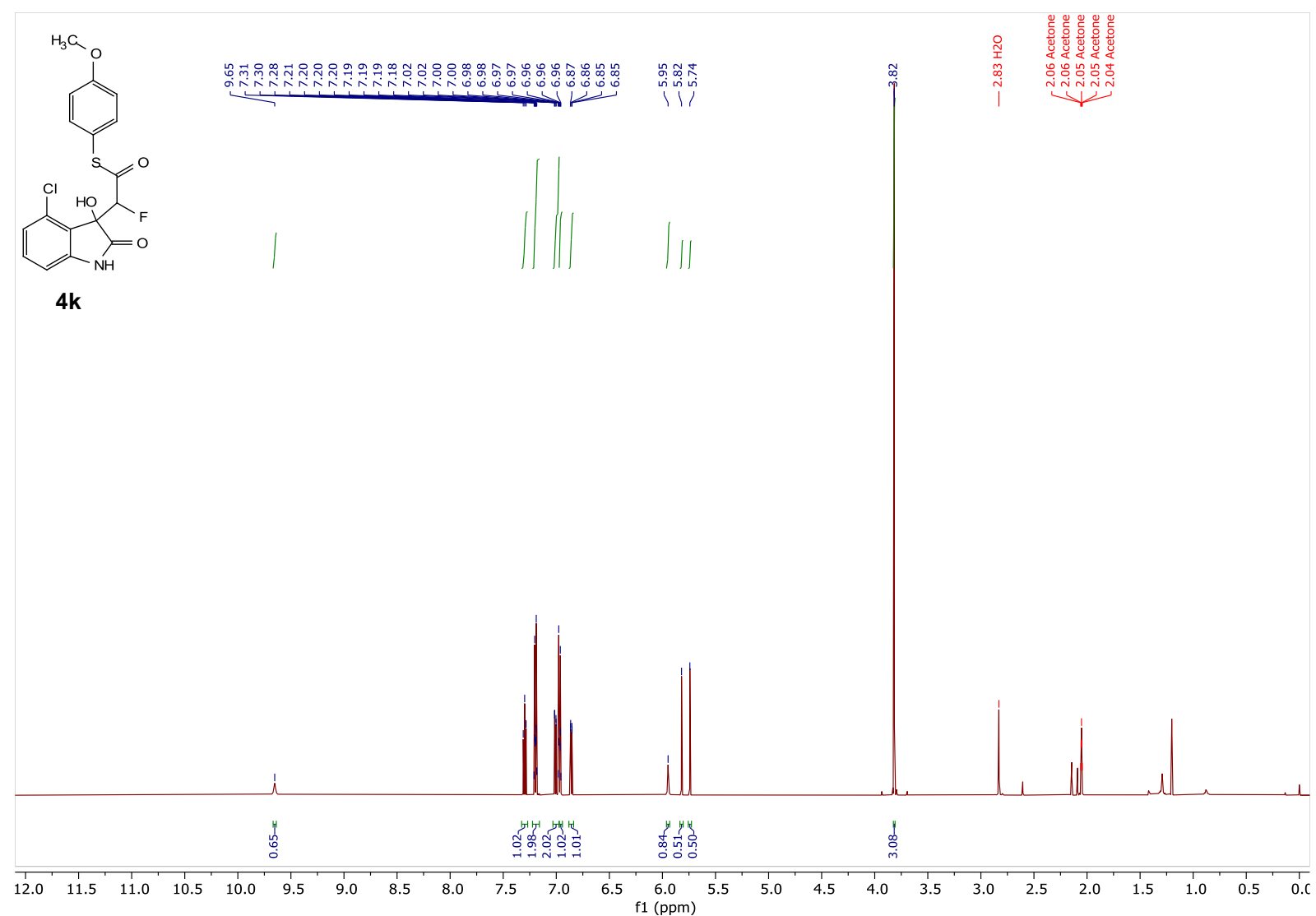
4k - ${ }^{13} \mathbf{C}$ NMR (151 MHz, d6-acetone)

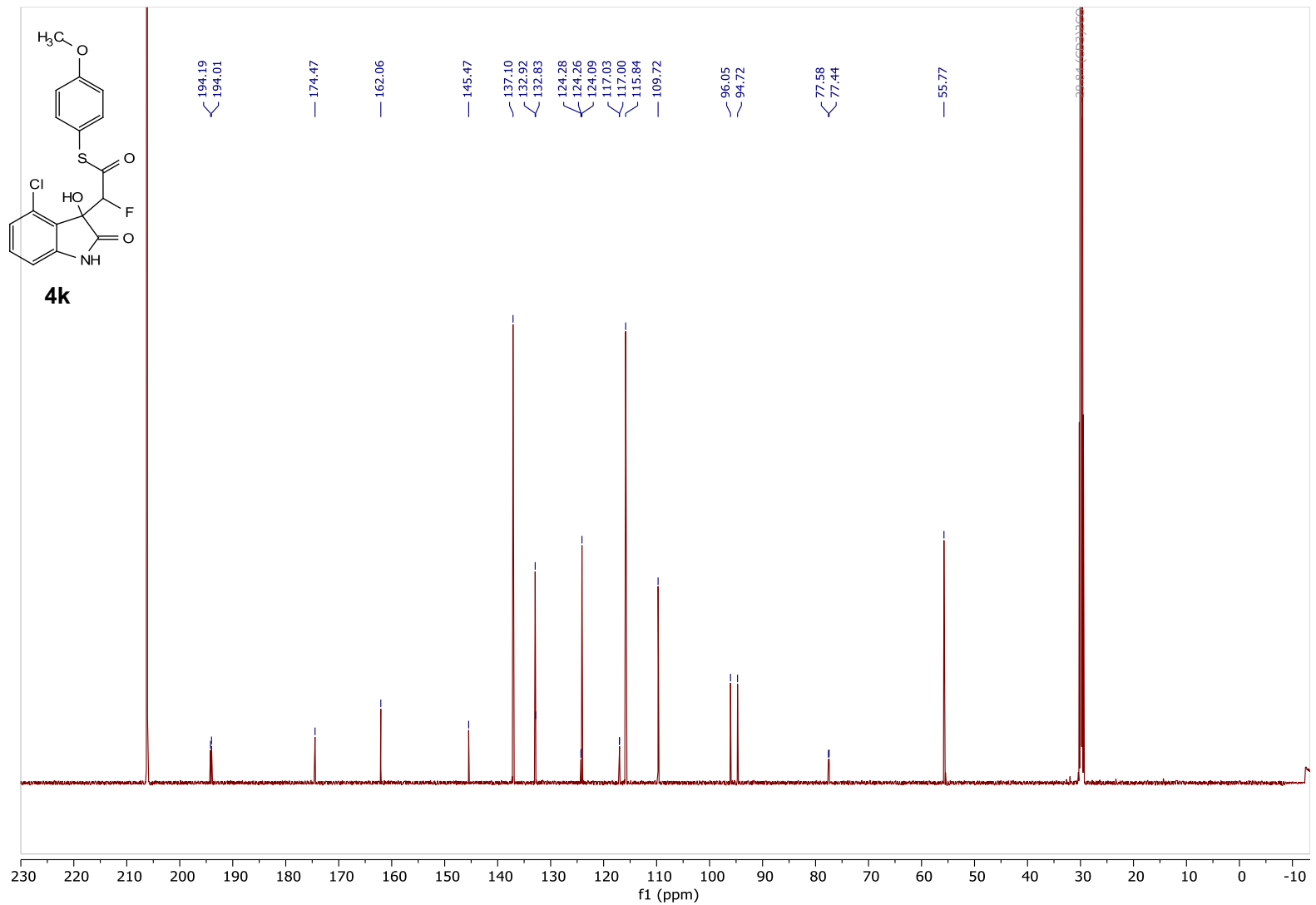

4k - ${ }^{19}$ F NMR (470 MHz, d6-acetone)

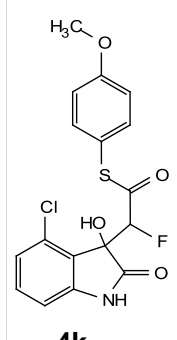

$4 k$ 
AI - ${ }^{1} \mathbf{H}$ NMR (500 MHz, $\left.\mathrm{CD}_{2} \mathrm{Cl}_{2}\right)$

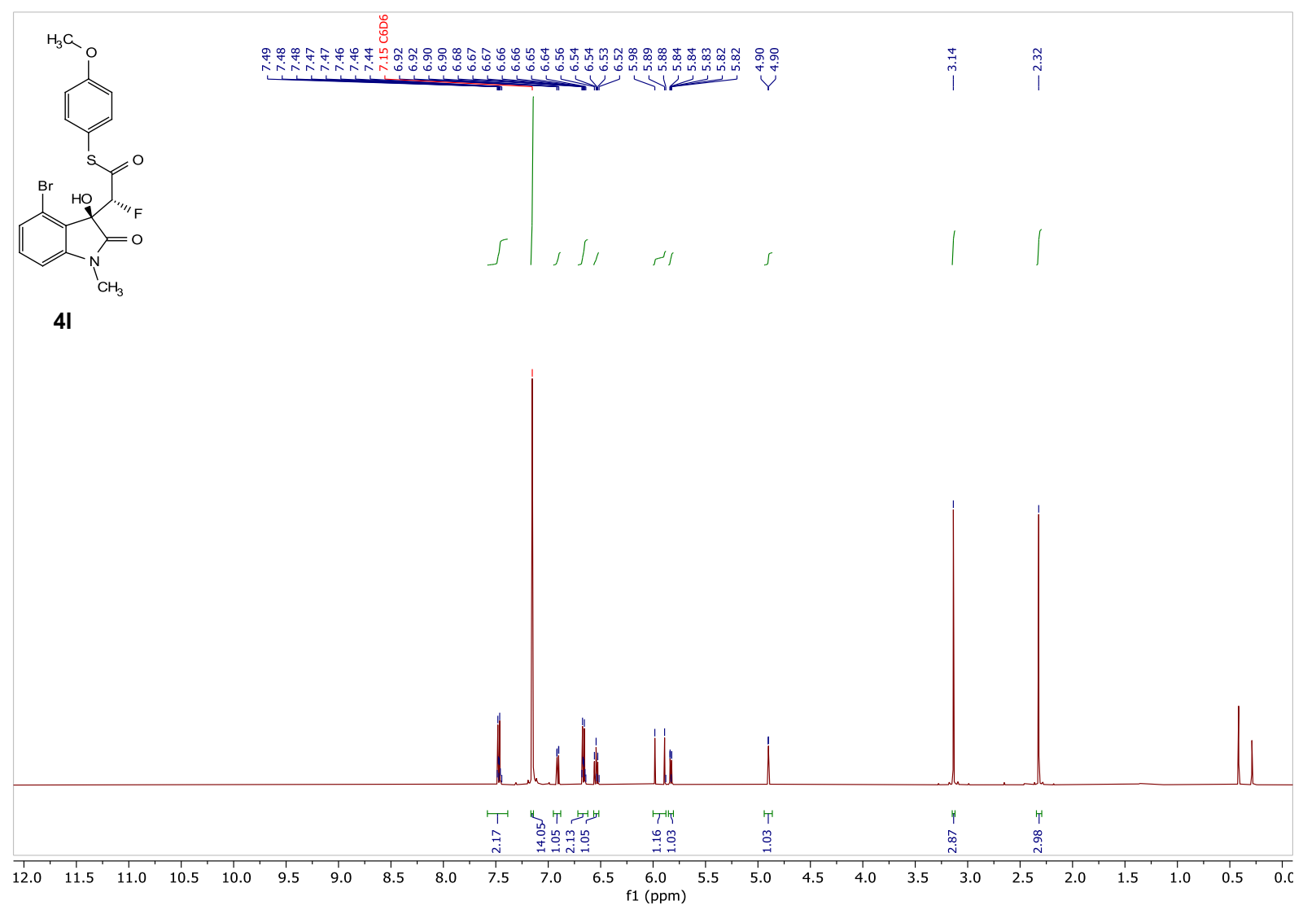

II - ${ }^{13} \mathrm{C}$ NMR (126 MHz, $\mathrm{CD}_{2} \mathrm{Cl}_{2}$ )

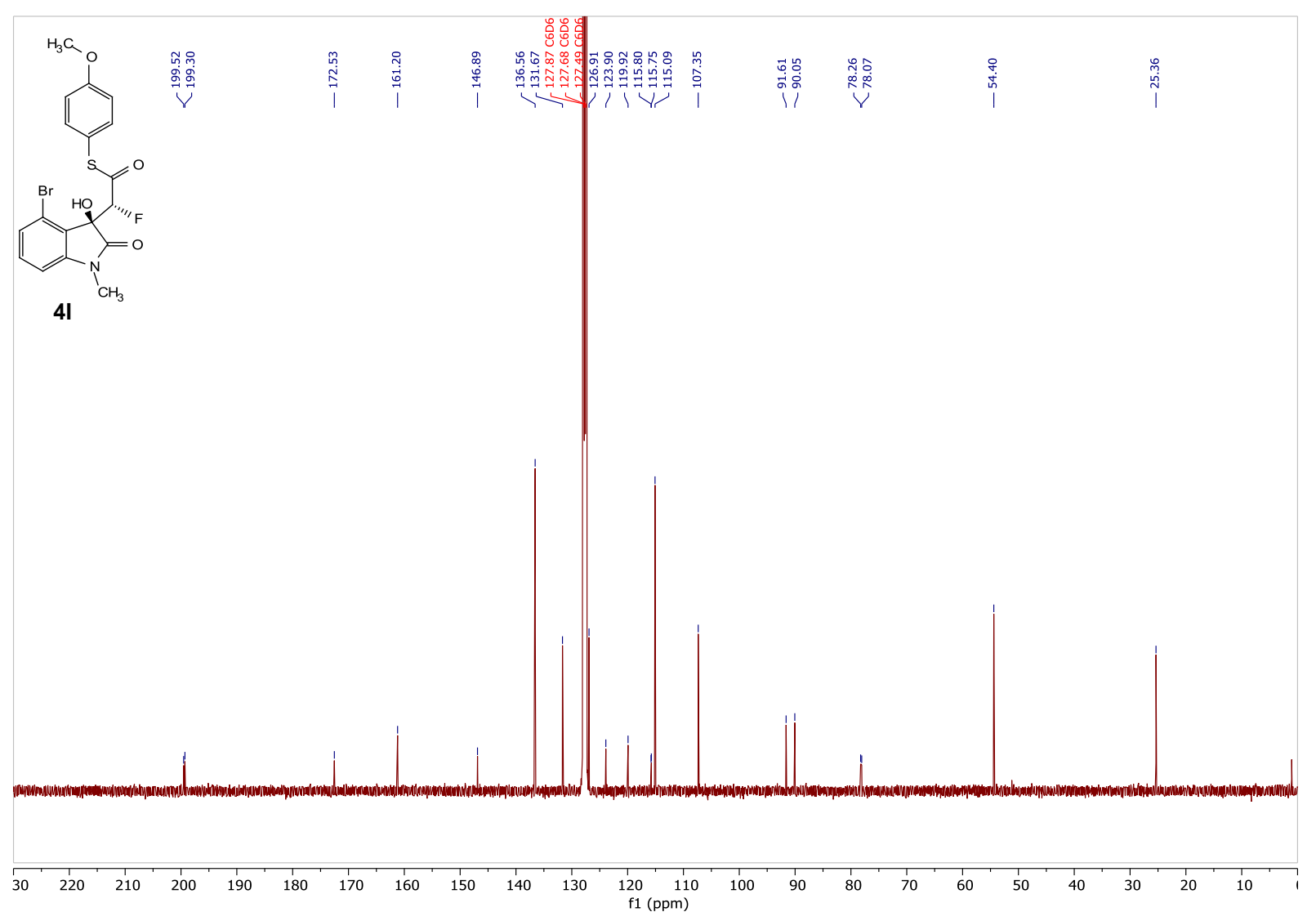


LI - ${ }^{19} \mathrm{~F}$ NMR (471 MHz, $\left.\mathrm{CD}_{2} \mathrm{Cl}_{2}\right)$

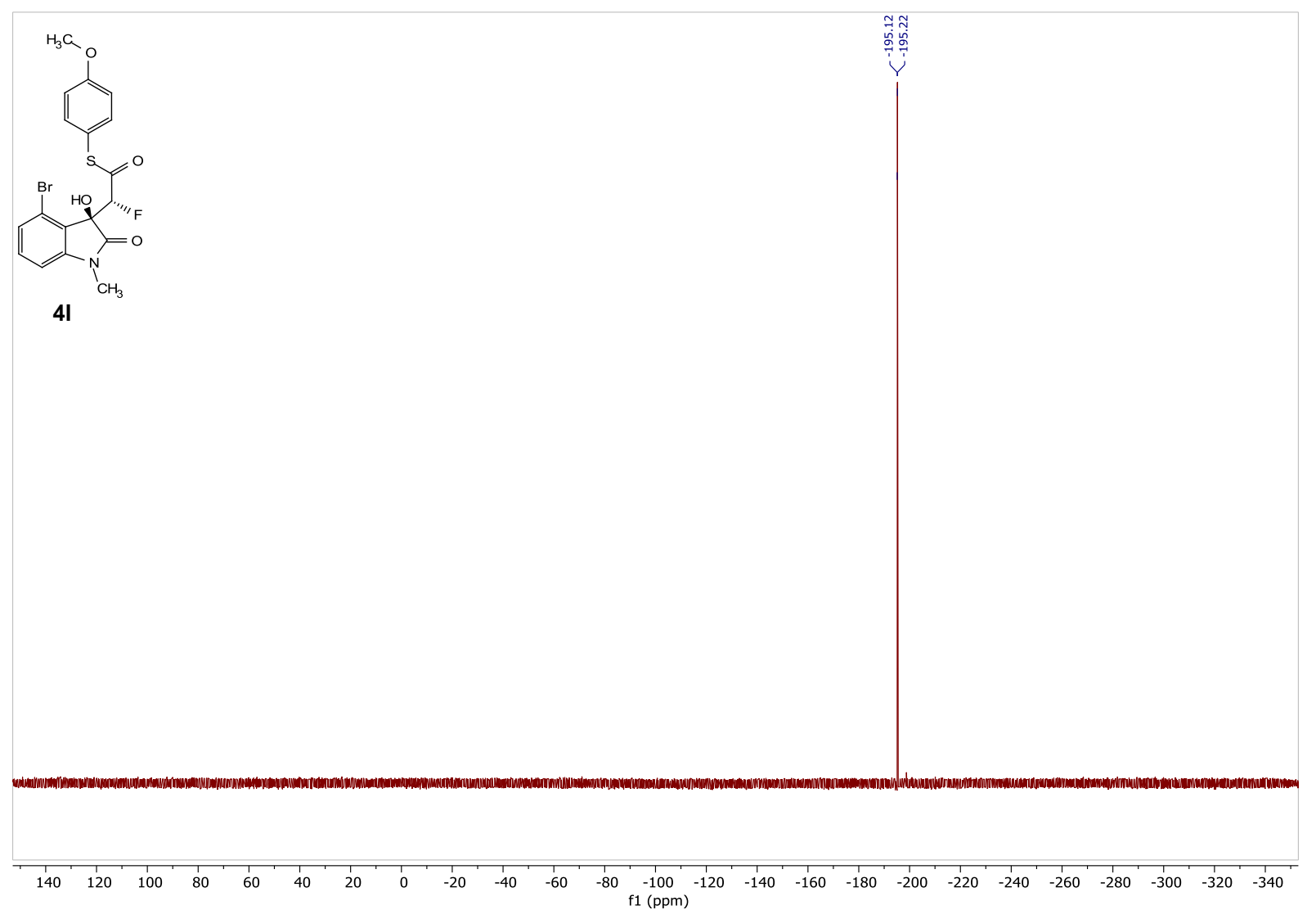

4m - ${ }^{1} \mathbf{H}$ NMR $\left(600 \mathrm{MHz}, \mathrm{C}_{6} \mathrm{D}_{6}\right)$

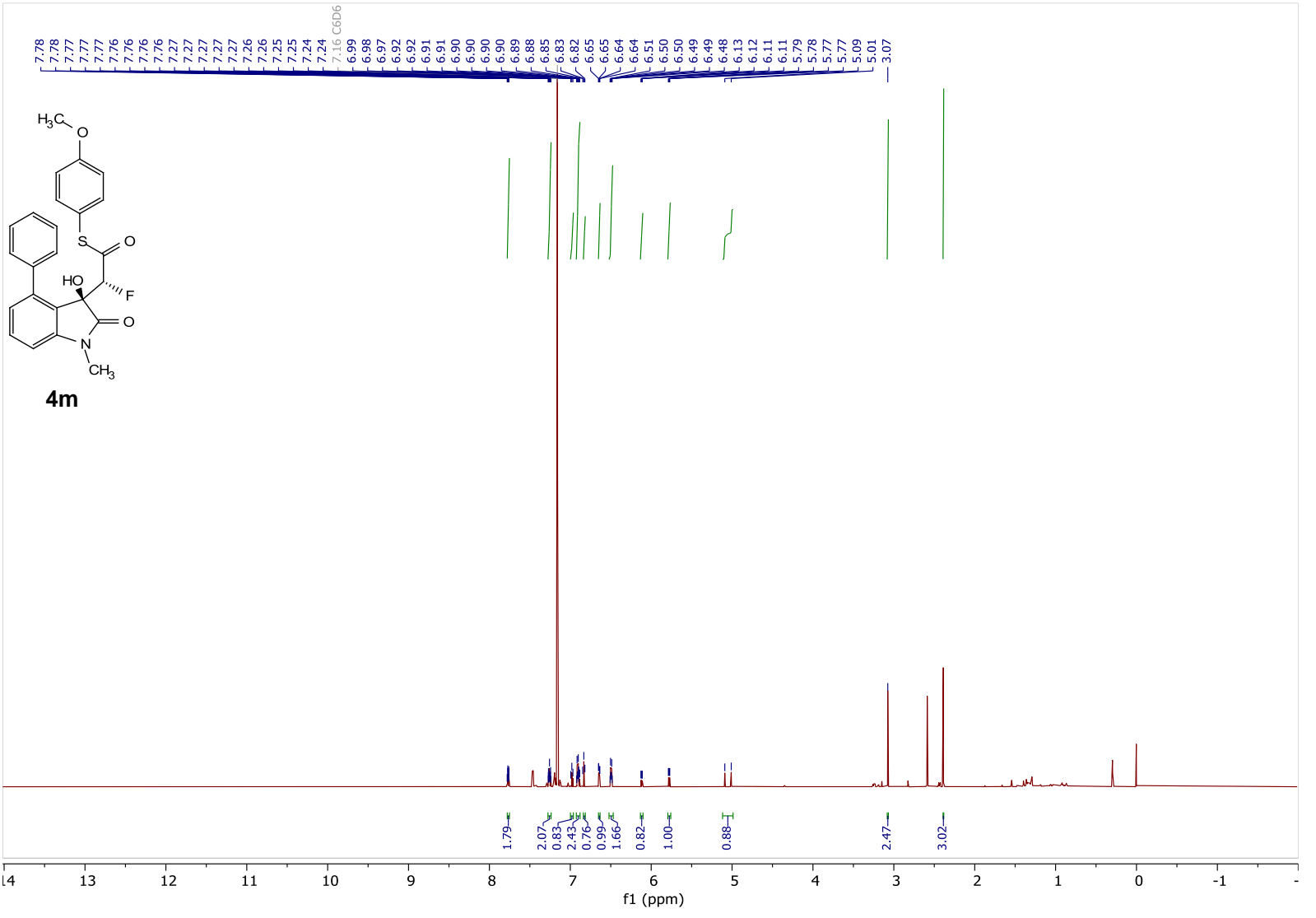




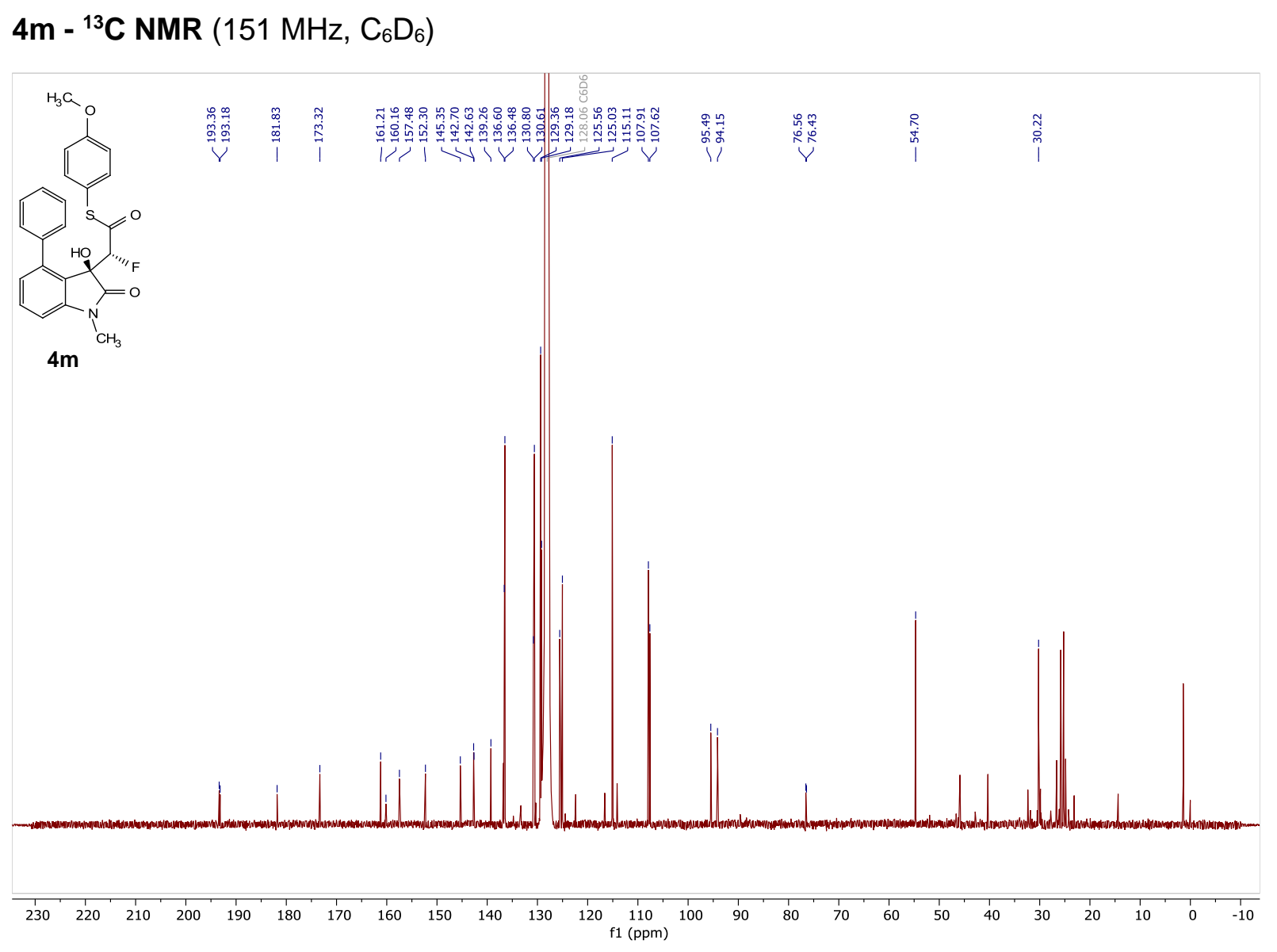

Um - ${ }^{19}$ F NMR $\left(470 \mathrm{MHz}, \mathrm{C}_{6} \mathrm{D}_{6}\right)$

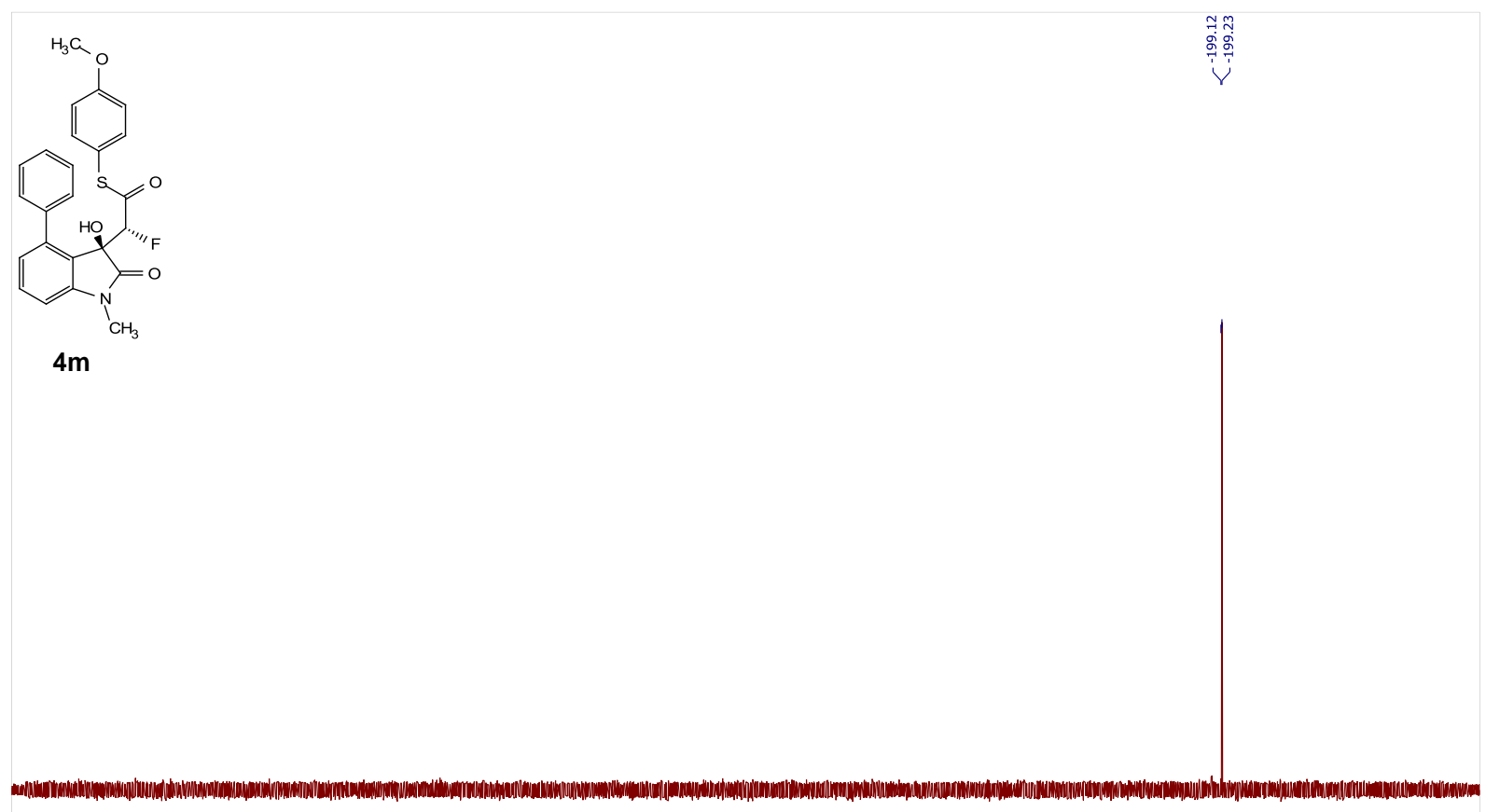

\begin{tabular}{llllllllllllllllllllllllllllllllllllllllllllll}
\hline & -75 & -80 & -85 & -90 & -95 & -100 & -105 & -110 & -115 & -120 & -125 & -130 & -135 & -140 & -145 & -150 & -155 & -160 & -165 & -170 & -175 & -180 & -185 & -190 & -195 & -200 & -205 & -210 & -215 & -220 & -22
\end{tabular} 
4n - ${ }^{1} \mathrm{H}$ NMR $\left(400 \mathrm{MHz}, \mathrm{C}_{6} \mathrm{D}_{6}\right)$

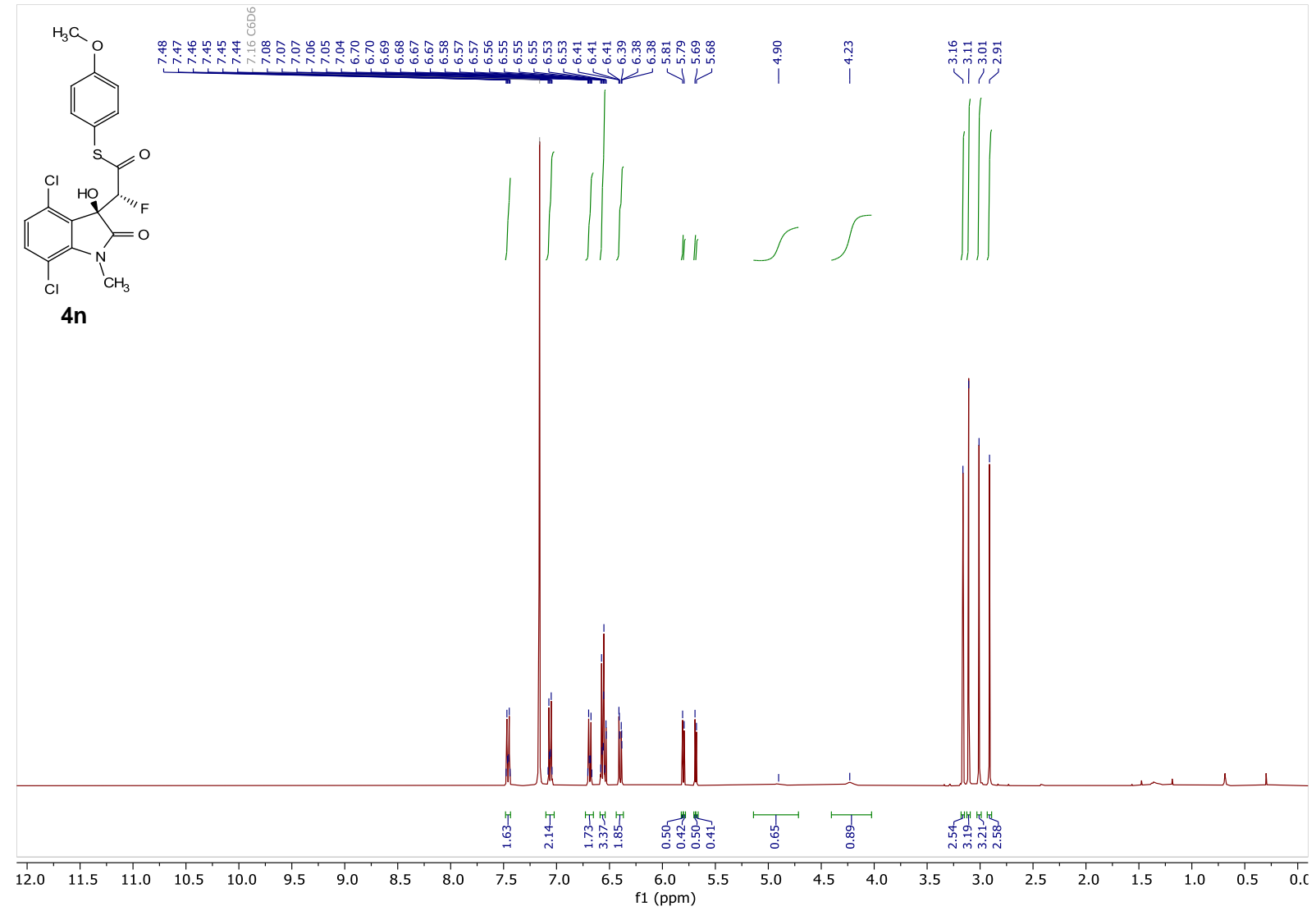

4n $-{ }^{13} \mathrm{C}$ NMR $\left(101 \mathrm{MHz}, \mathrm{C}_{6} \mathrm{D}_{6}\right)$

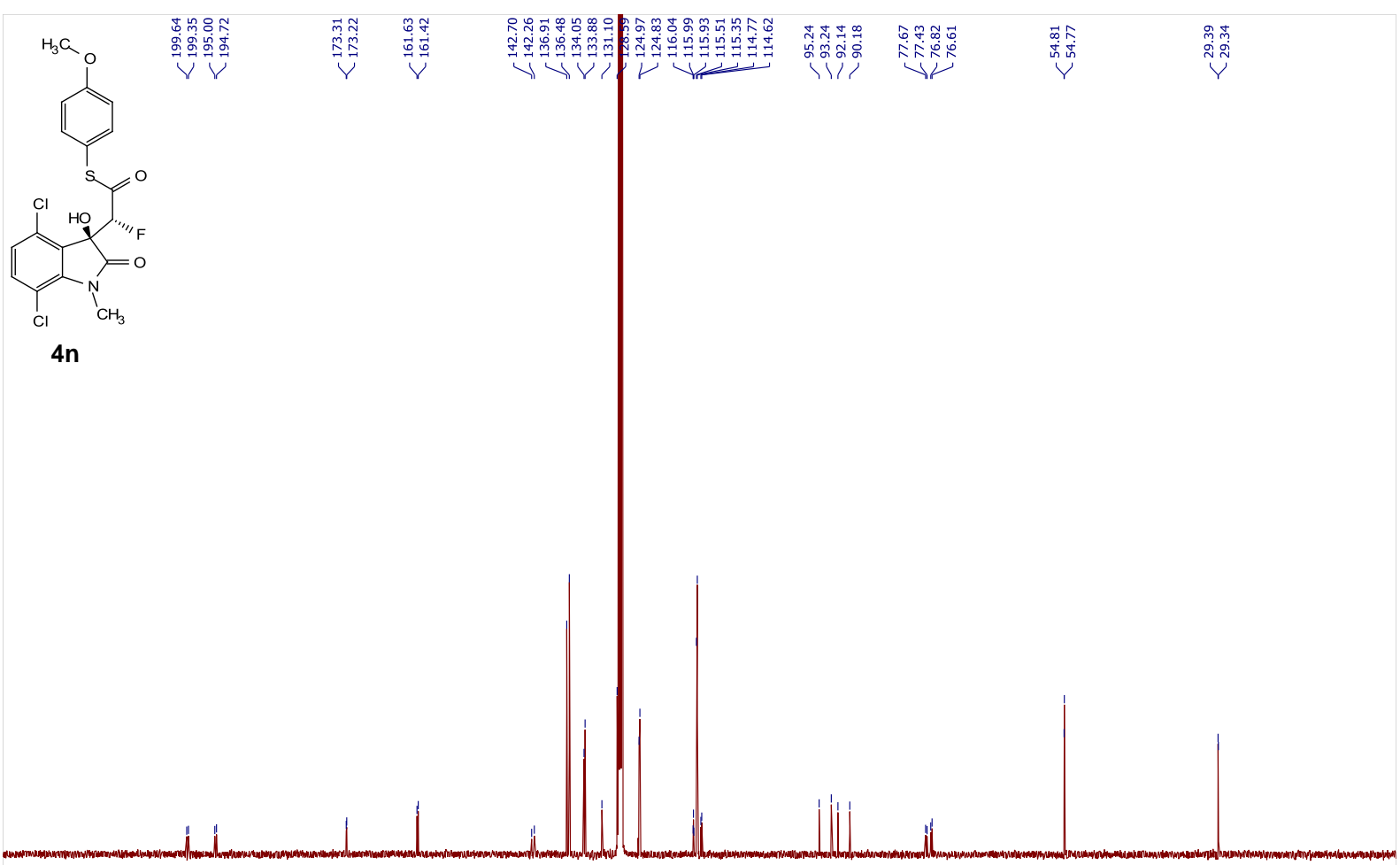

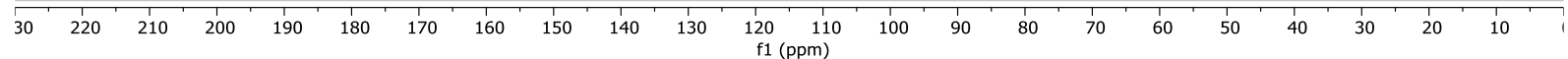


Un - ${ }^{19}$ F NMR (376 MHz, $\left.\mathrm{C}_{6} \mathrm{D}_{6}\right)$

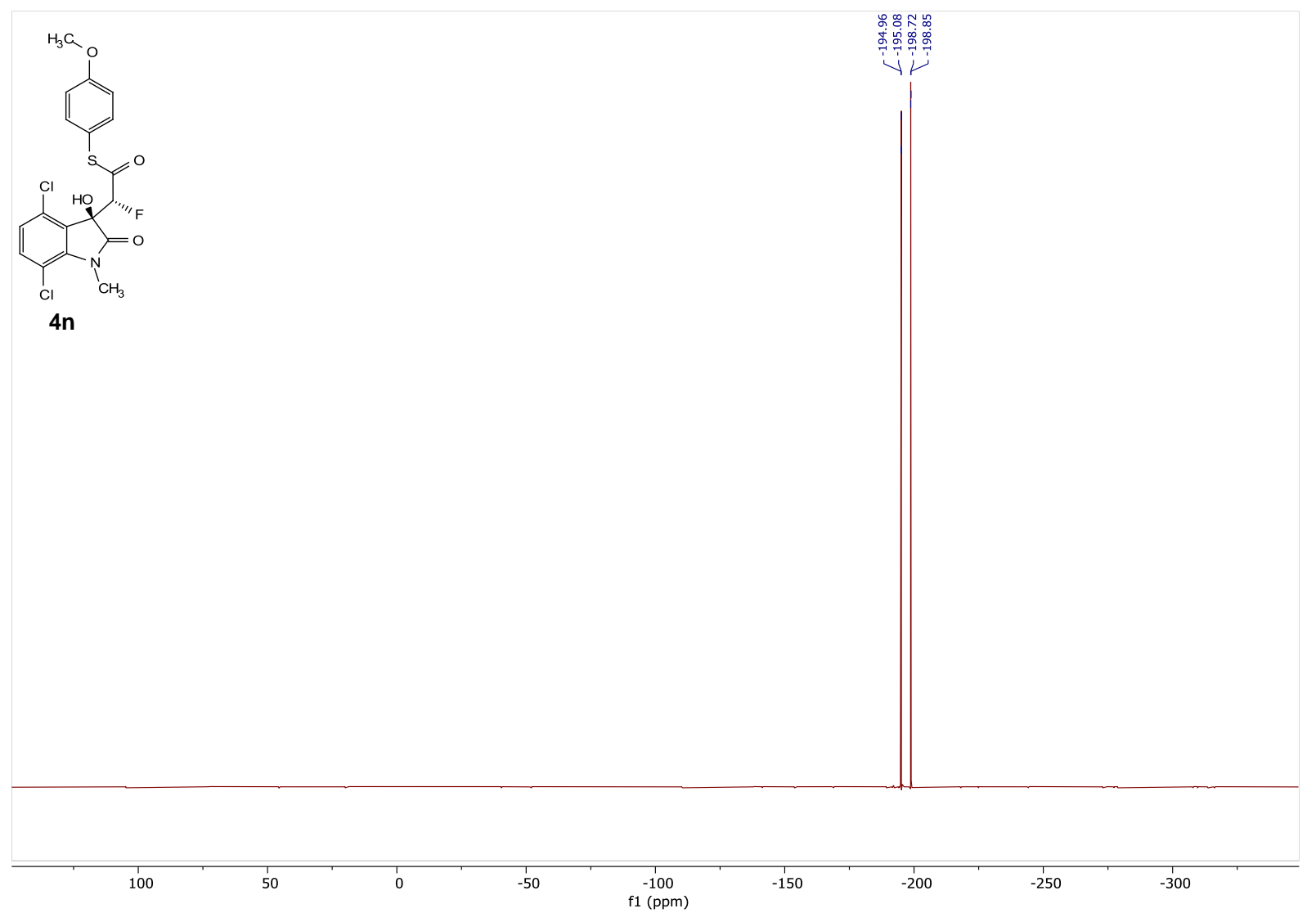

40 - ${ }^{1} \mathbf{H}$ NMR (400 MHz, $\left.\mathrm{CD}_{2} \mathrm{Cl}_{2}\right)$

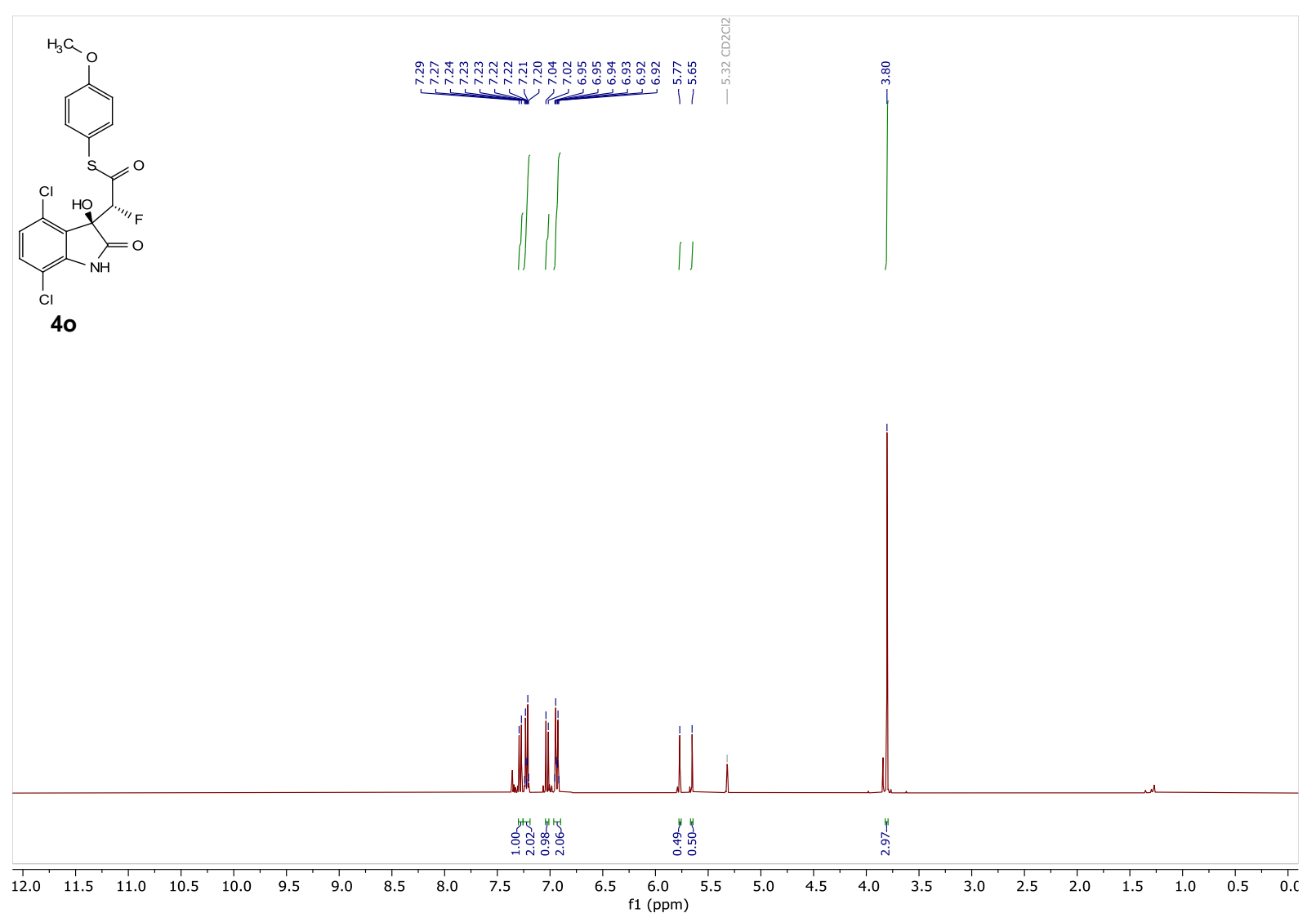


4o - ${ }^{13}$ C NMR (101 MHz, $\mathrm{CD}_{2} \mathrm{Cl}_{2}$ )

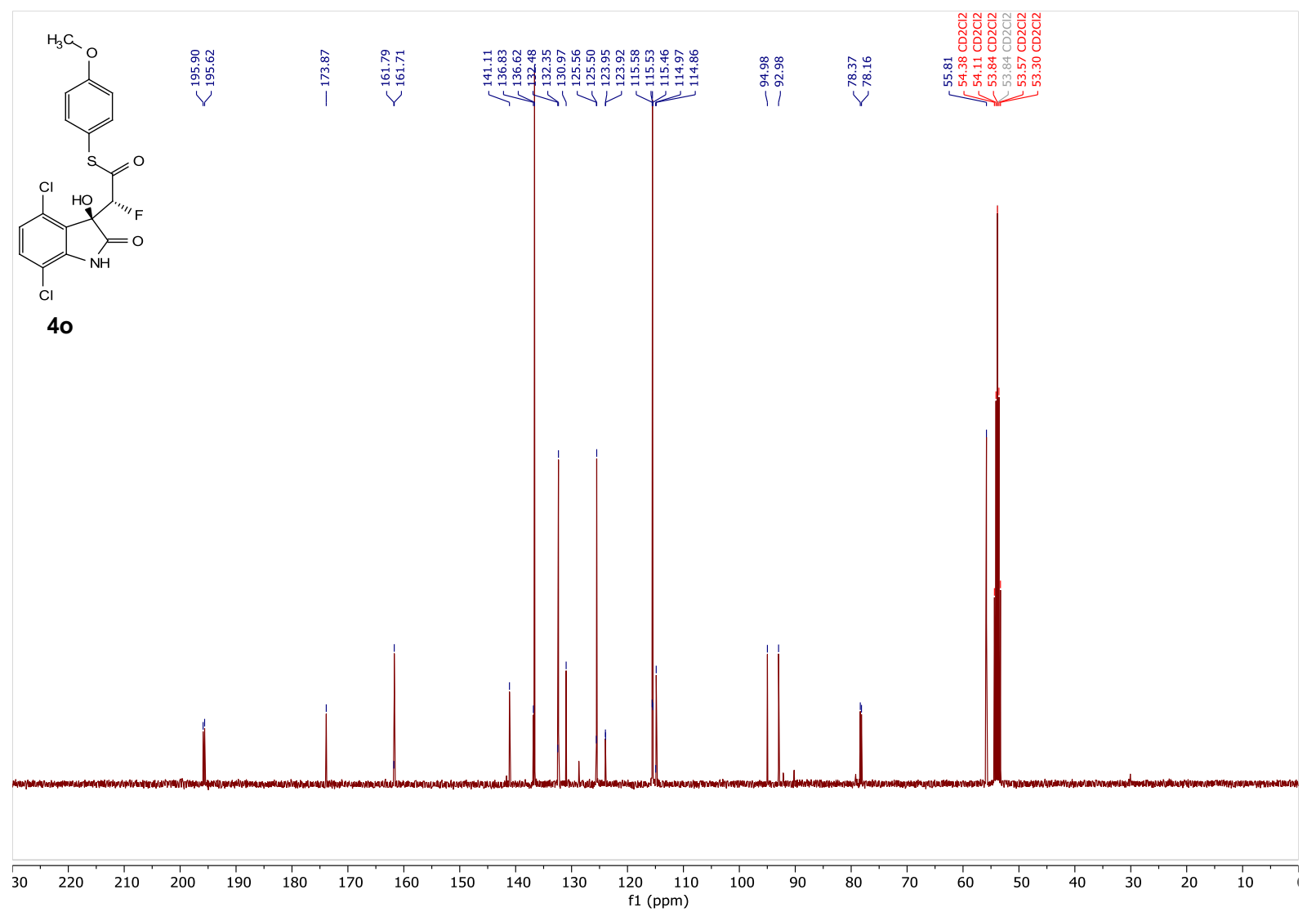

40 - ${ }^{19}$ F NMR (376 MHz, $\mathrm{CD}_{2} \mathrm{Cl}_{2}$ )

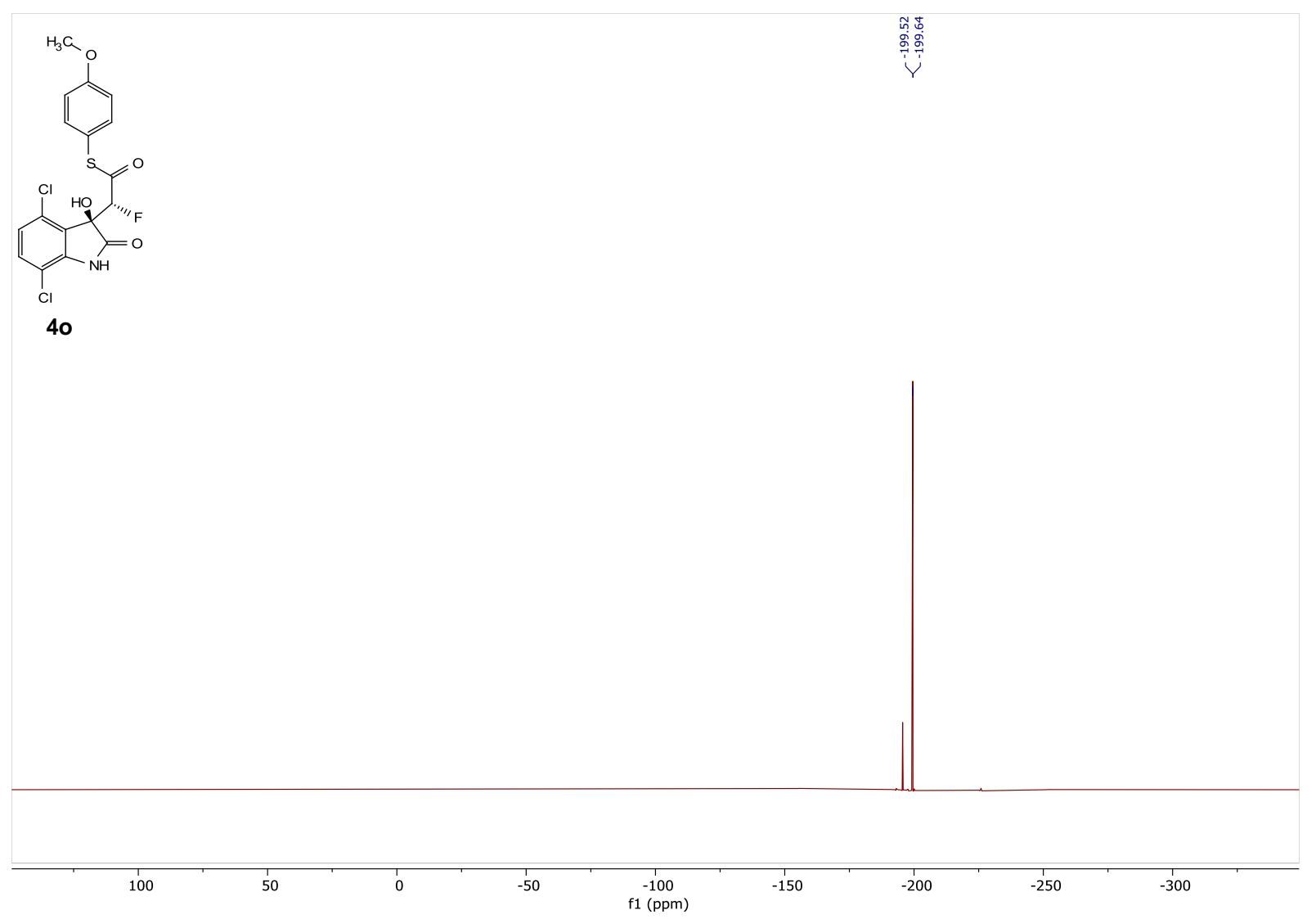


40 - Other Diastereoisomer- ${ }^{1} \mathrm{H}$ NMR $\left(400 \mathrm{MHz}, \mathrm{CD}_{2} \mathrm{Cl}_{2}\right)$

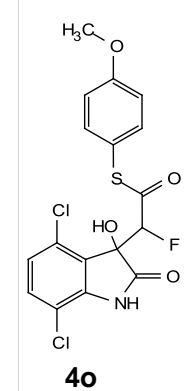

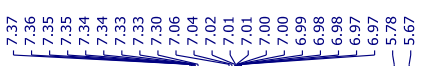
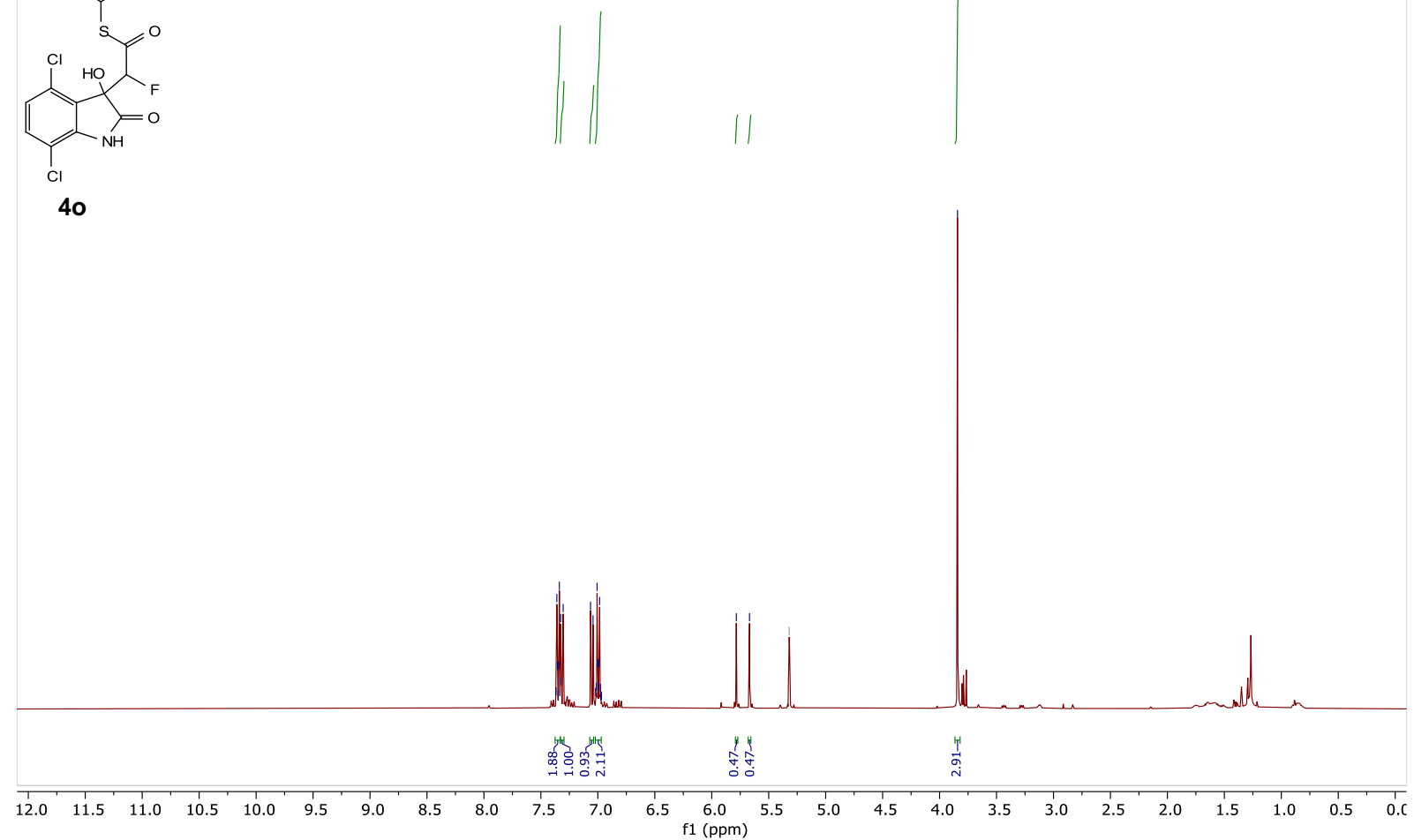

$40-{ }^{13} \mathrm{C}$ NMR $\left(101 \mathrm{MHz}, \mathrm{CD}_{2} \mathrm{Cl}_{2}\right)$

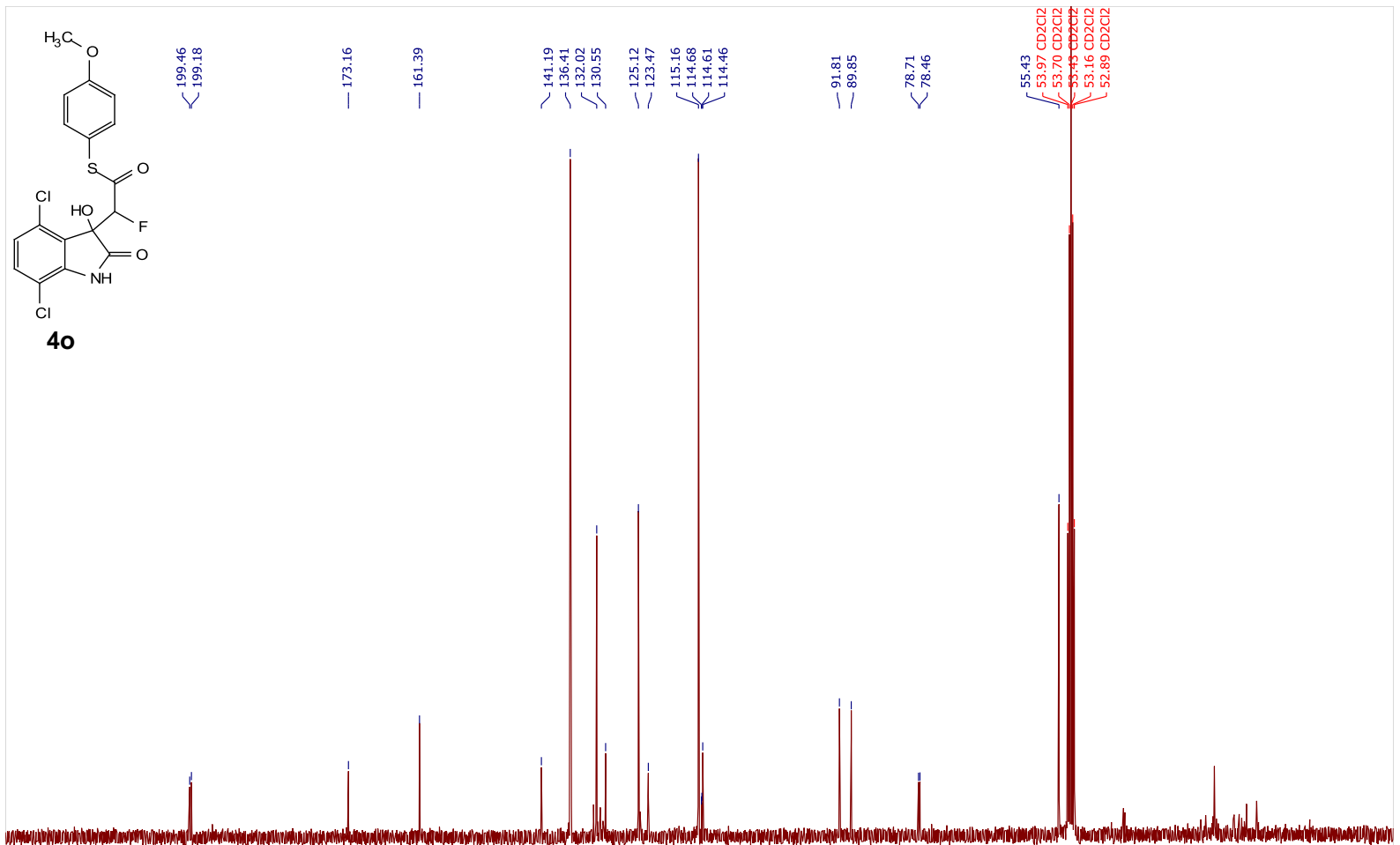

$\begin{array}{rllllllllll}220 & 210 & 200 & 190 & 180 & 170 & 160 & 150 & 140 & 130 & 120 \\ \mathrm{f} 1(\mathrm{ppm})\end{array}$ 
4o - ${ }^{19}$ F NMR (376 MHz, $\left.\mathrm{CD}_{2} \mathrm{Cl}_{2}\right)$

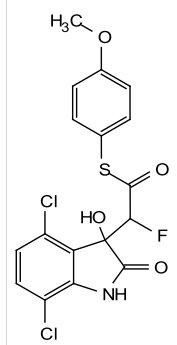

40$$
40
$$

100

50

$-50$

$\stackrel{-100}{f 1(p p m)}$

$-150 \quad-200$

$-250$

$-300$

4p - ${ }^{1} \mathrm{H}$ NMR $\left(400 \mathrm{MHz}, \mathrm{C}_{6} \mathrm{D}_{6}\right)$

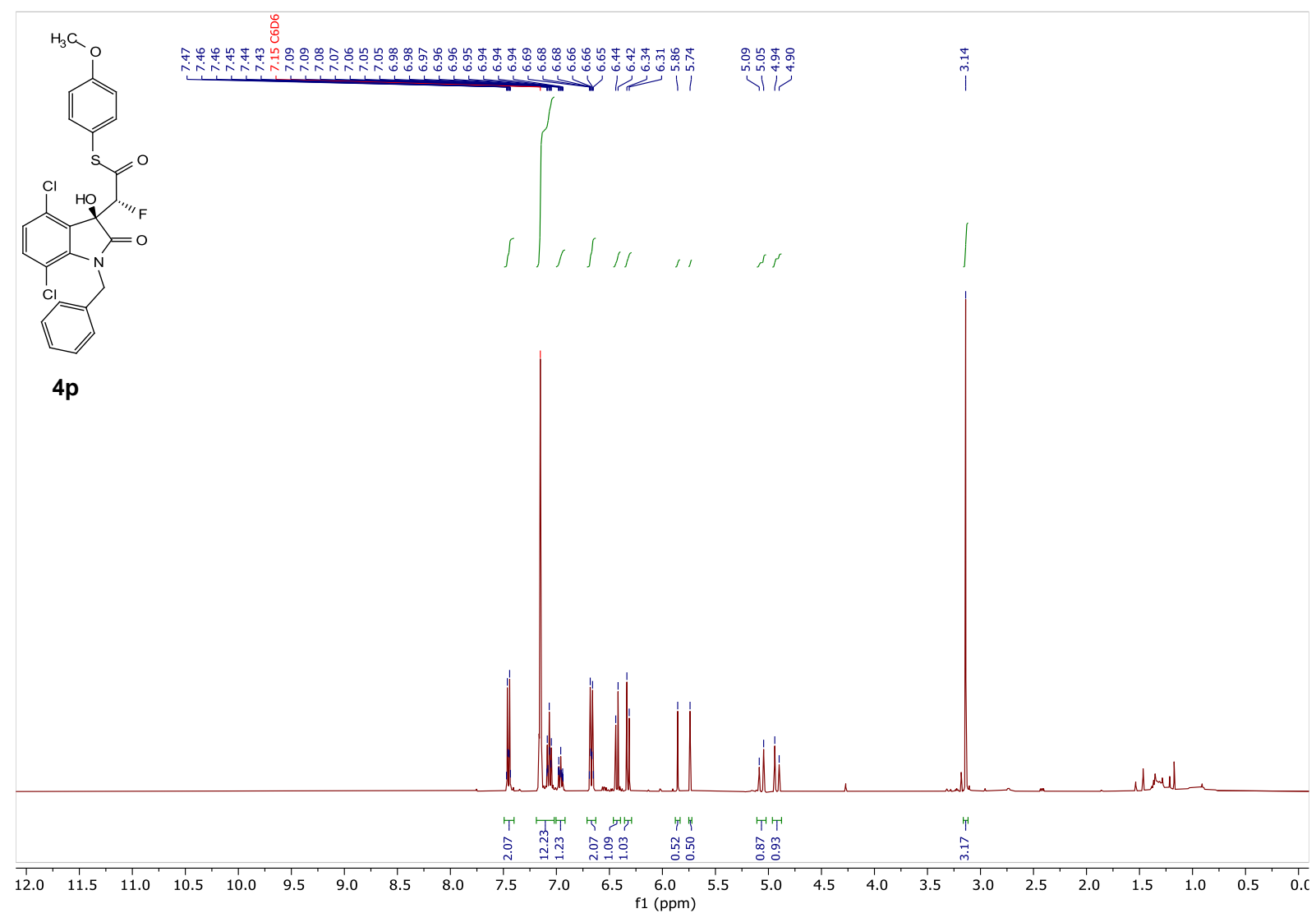


Ap - ${ }^{13}$ C NMR (101 MHz, $\left.C_{6} D_{6}\right)$

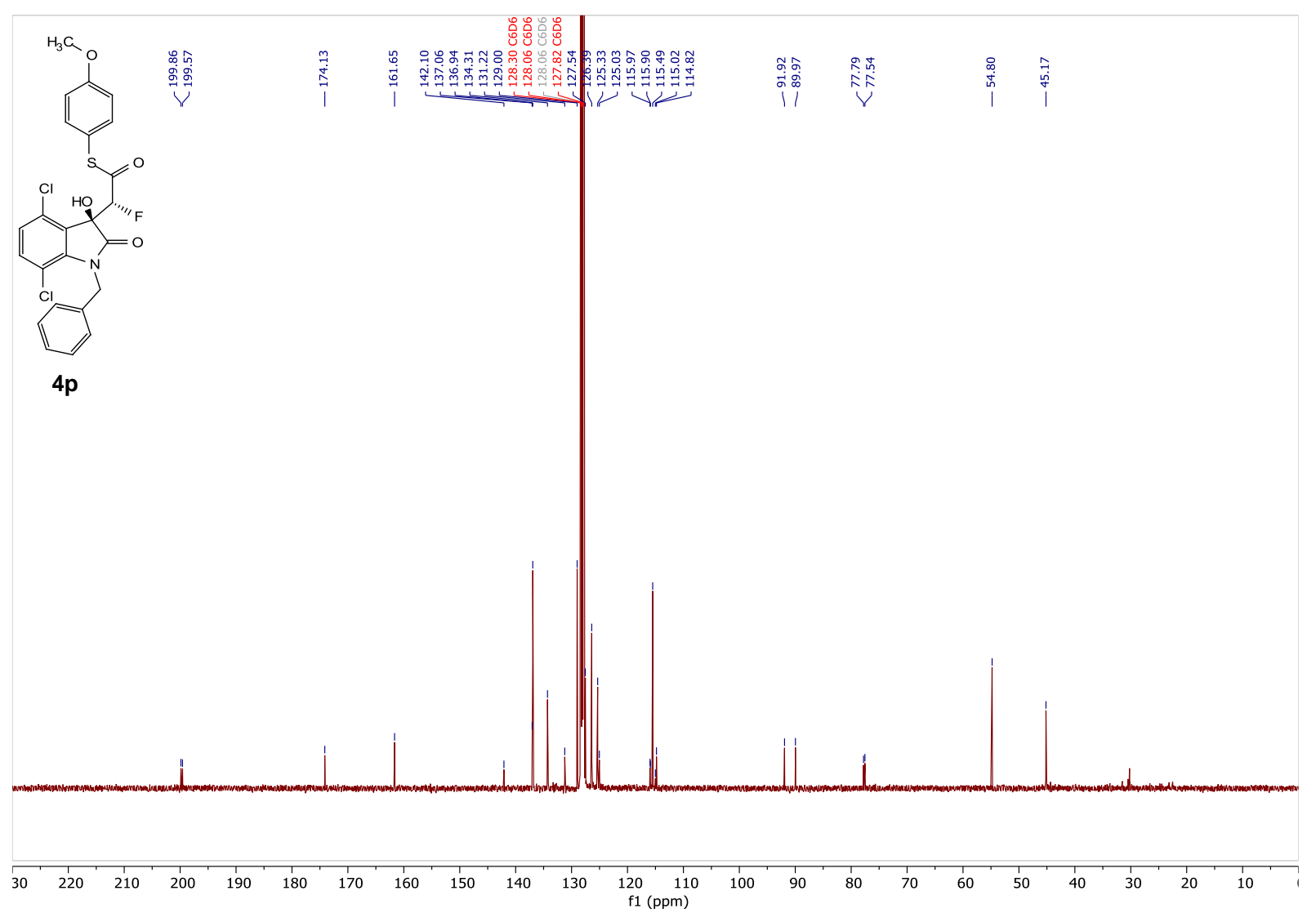

Ap - ${ }^{19}$ F NMR (376 MHz, $\left.\mathrm{C}_{6} \mathrm{D}_{6}\right)$

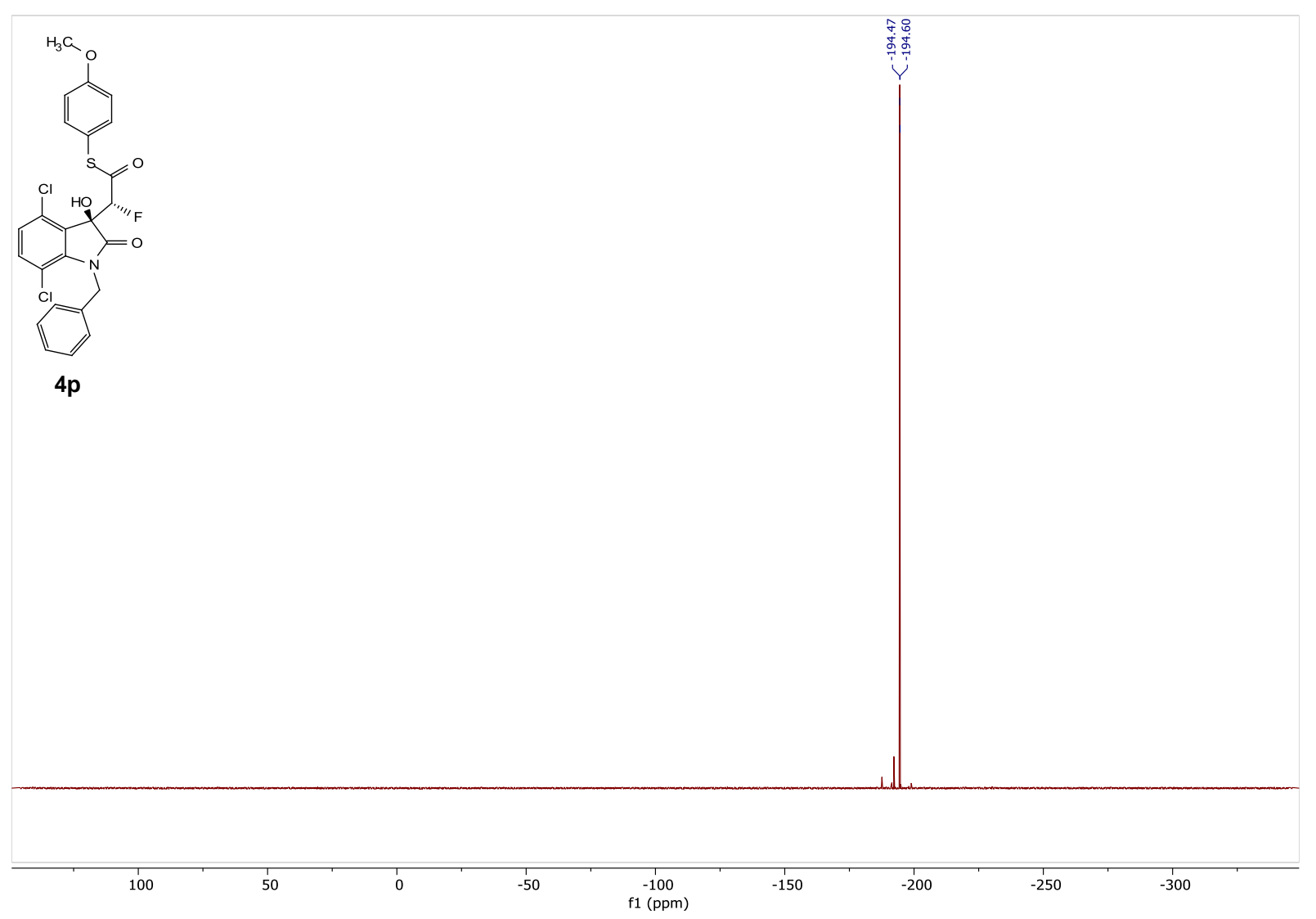


$4 \mathbf{p}$ - Other Diastereoisomer - ${ }^{1} \mathrm{H}$ NMR $\left(400 \mathrm{MHz}, \mathrm{C}_{6} \mathrm{D}_{6}\right)$

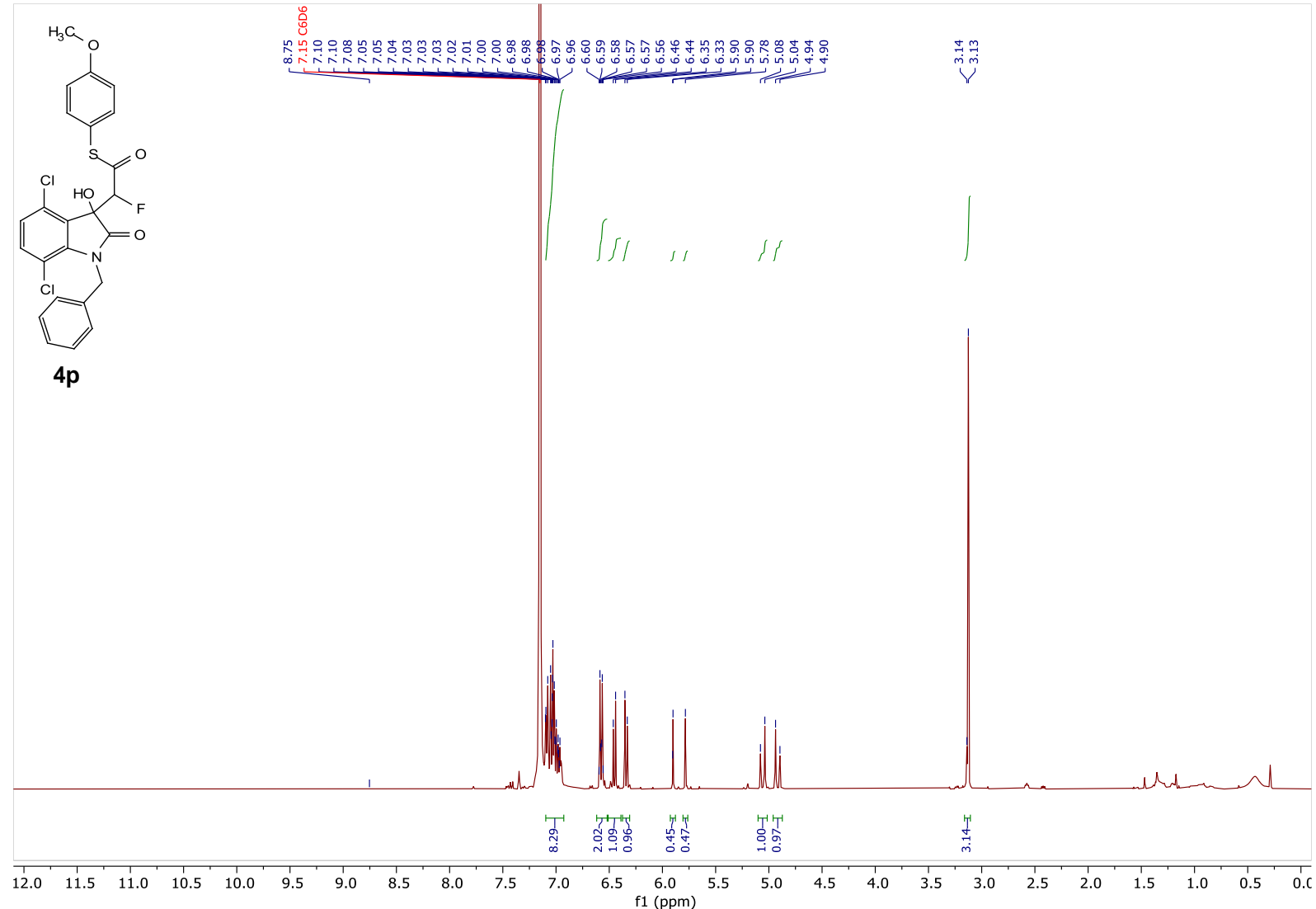

4p - ${ }^{13}$ C NMR $\left(101 \mathrm{MHz}, \mathrm{C}_{6} \mathrm{D}_{6}\right)$

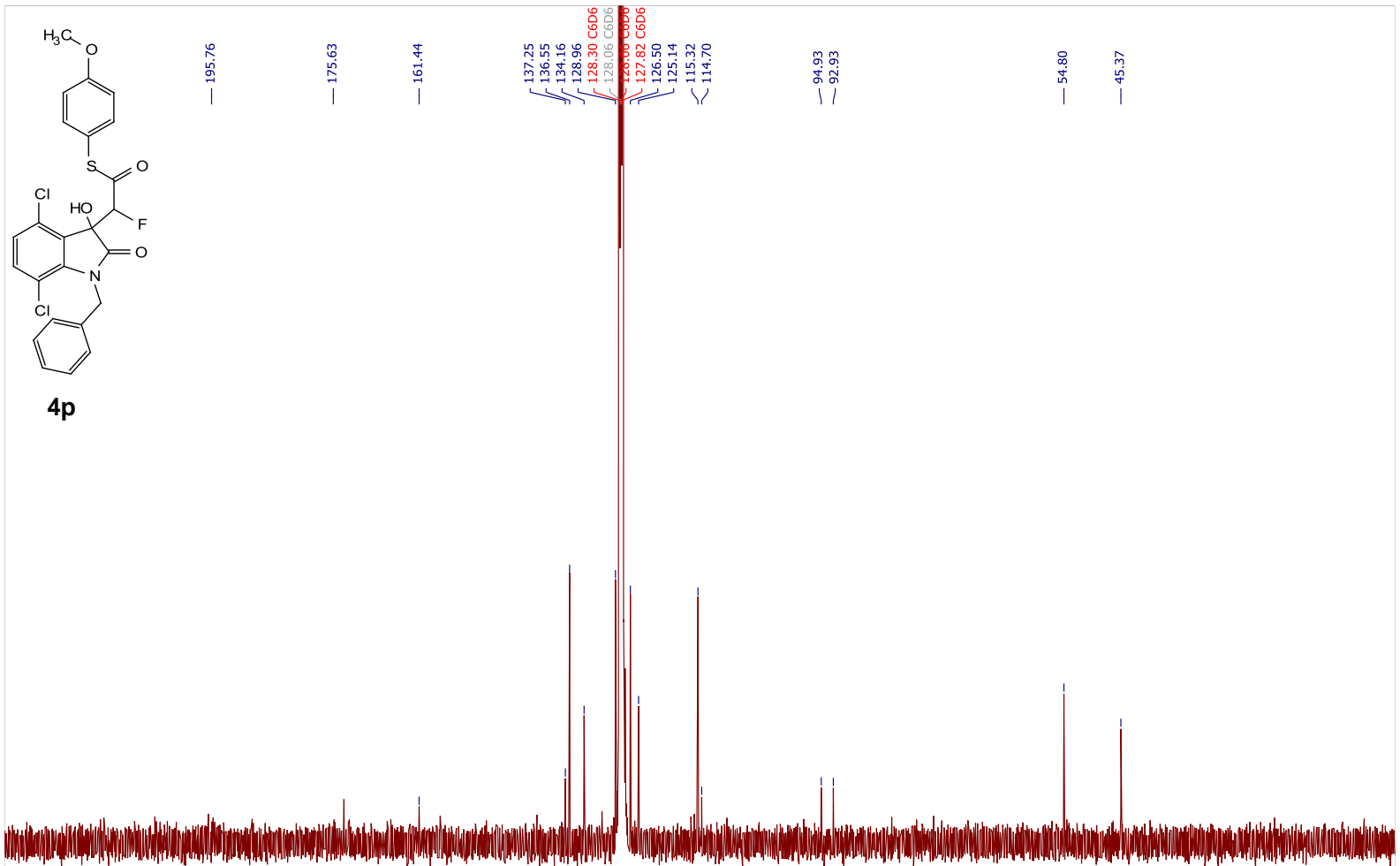

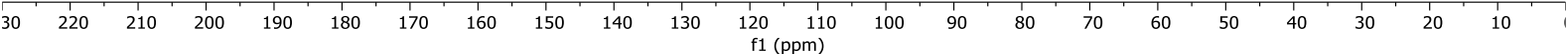


4p - ${ }^{19}$ F NMR (376 MHz, $\left.C_{6} D_{6}\right)$

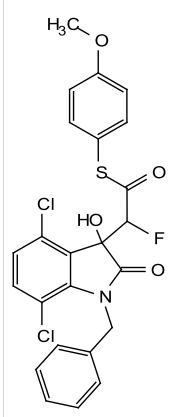

$4 p$

50

\begin{tabular}{llllllllllllllllllllllllllllllllllllll}
\hline & 30 & 20 & 10 & 0 & -10 & -20 & -30 & -40 & -50 & -60 & -70 & -80 & -90 & -100 & -110 & -120 & -130 & -140 & -150 & -160 & -170 & -180 & -190 & -200 & -210 & -220 & -230 & -240 & $-2 !$
\end{tabular} 4q - ${ }^{1} \mathrm{H}$ NMR $\left(400 \mathrm{MHz}, \mathrm{C}_{6} \mathrm{D}_{6}\right)$

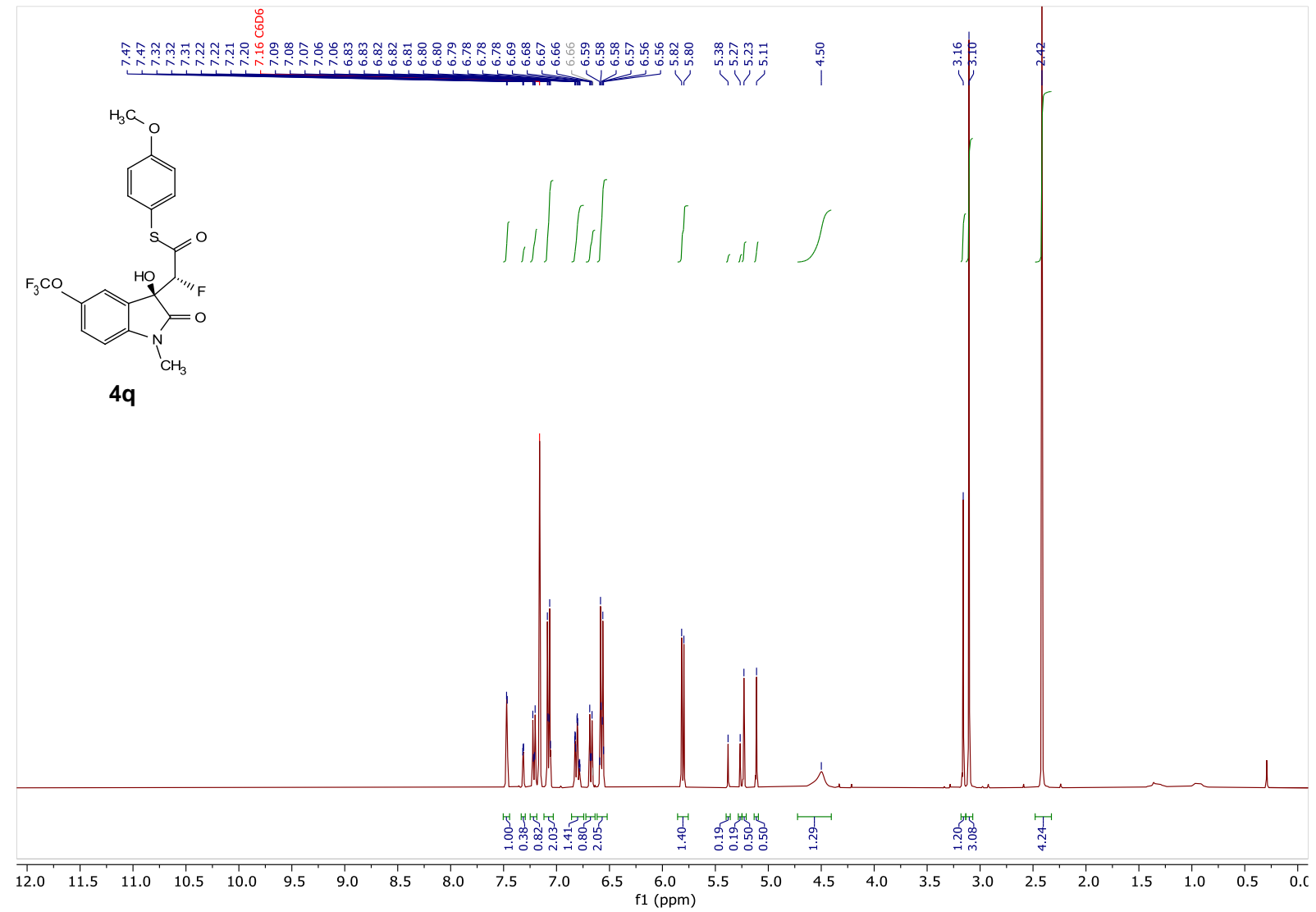


Aq - ${ }^{13}$ C NMR (101 MHz, $\left.C_{6} D_{6}\right)$

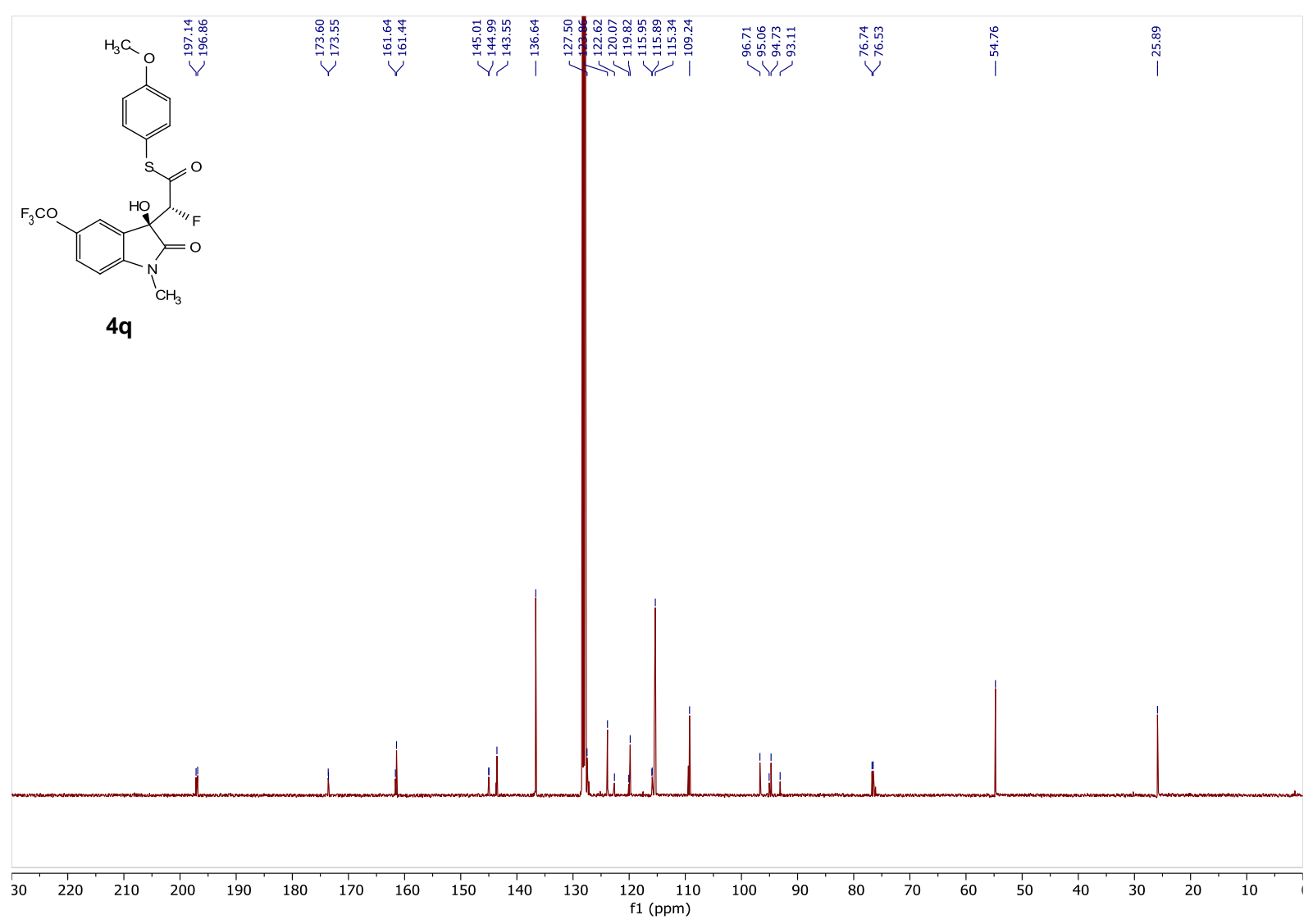

Aq - ${ }^{19}$ F NMR $\left(376 \mathrm{MHz}, \mathrm{C}_{6} \mathrm{D}_{6}\right)$

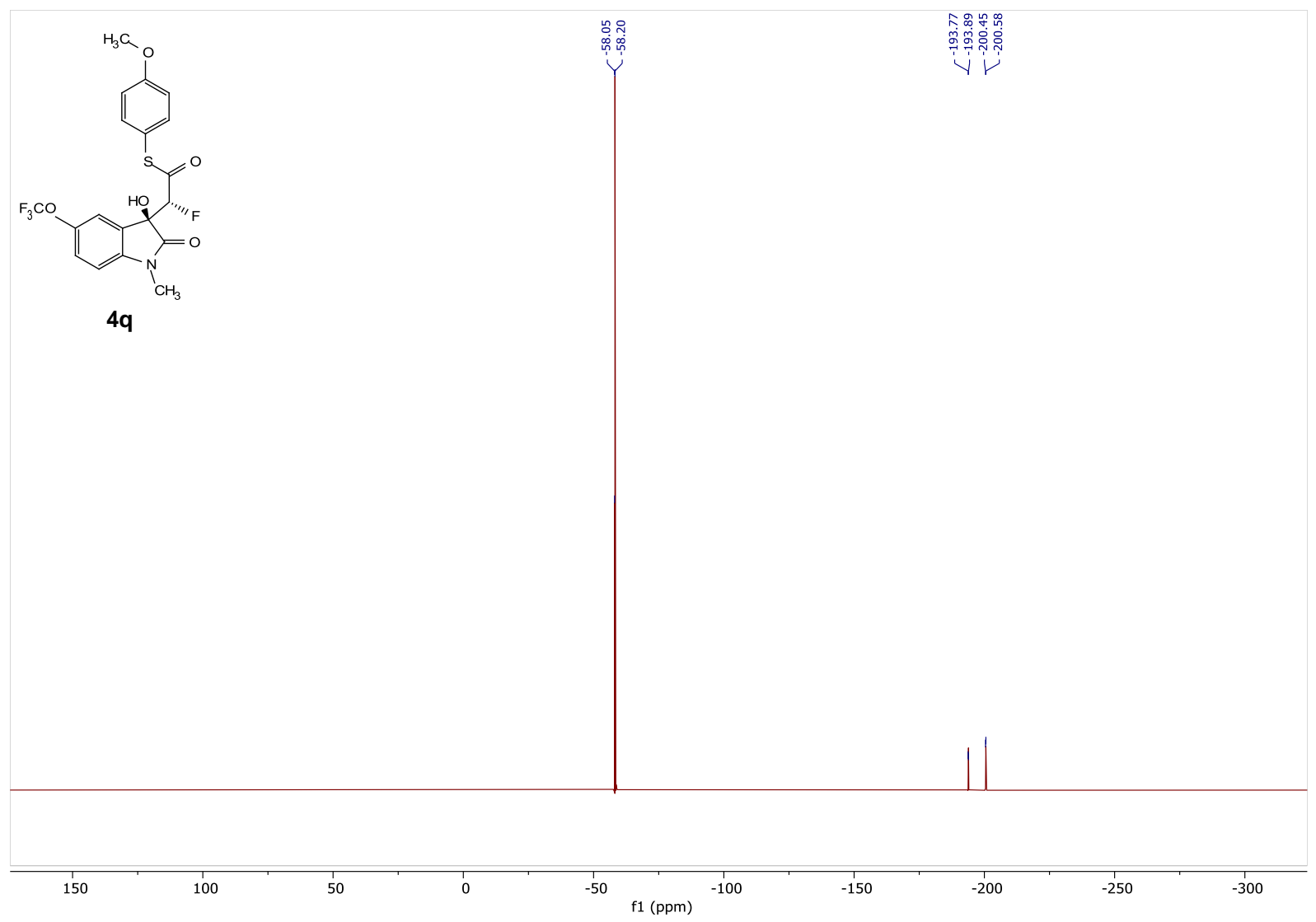




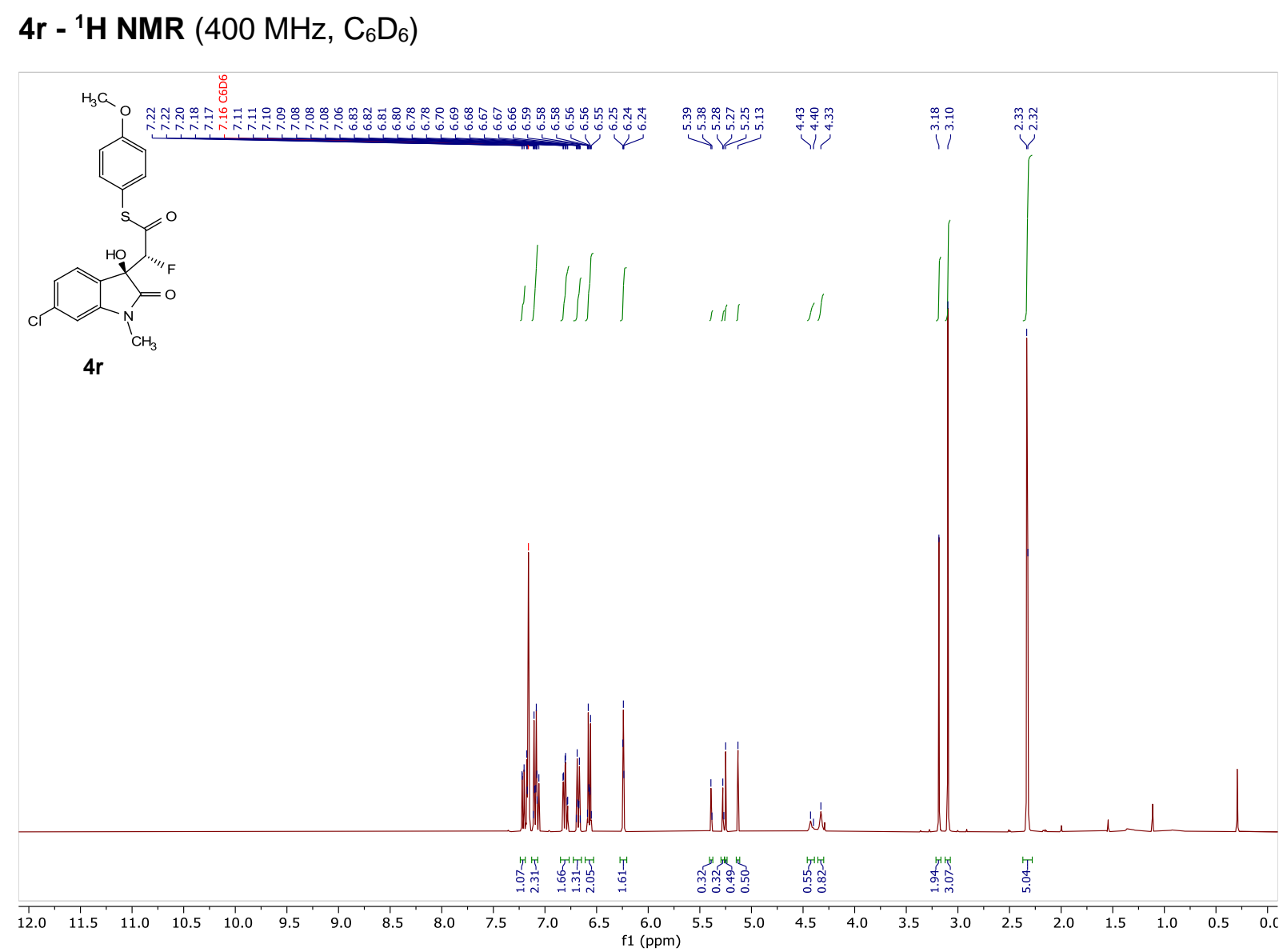

4r $-{ }^{13} \mathrm{C}$ NMR $\left(101 \mathrm{MHz}, \mathrm{C}_{6} \mathrm{D}_{6}\right)$

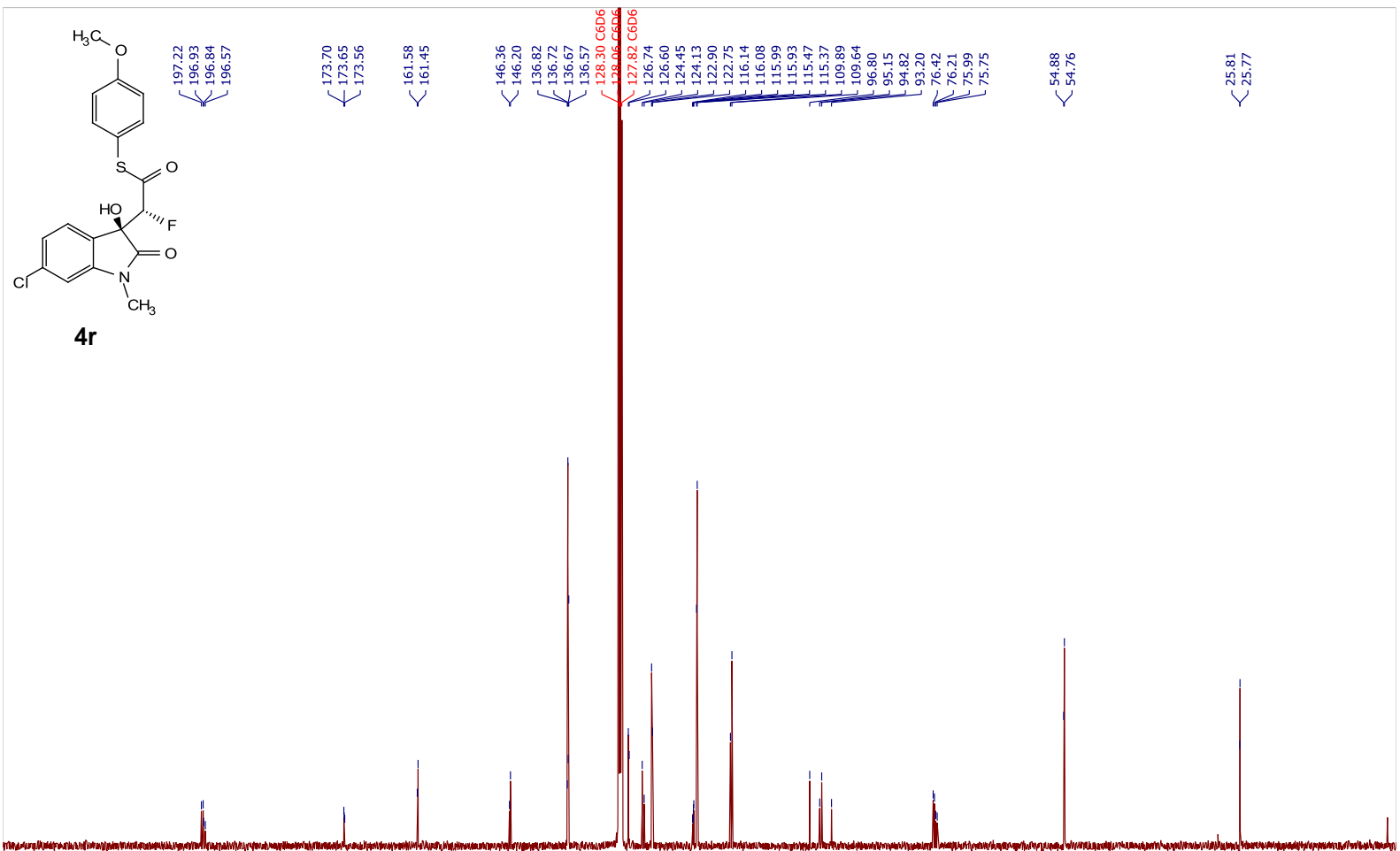

$\begin{array}{llllllllllllllllllllllllll}220 & 210 & 200 & 190 & 180 & 170 & 160 & 150 & 140 & 130 & 120 & 110 & 100 & 90 & 80 & 70 & 60 & 50 & 40 & 30 & 20 & 10 & 1\end{array}$ 
4r - ${ }^{19}$ F NMR (376 MHz, $\left.C_{6} D_{6}\right)$

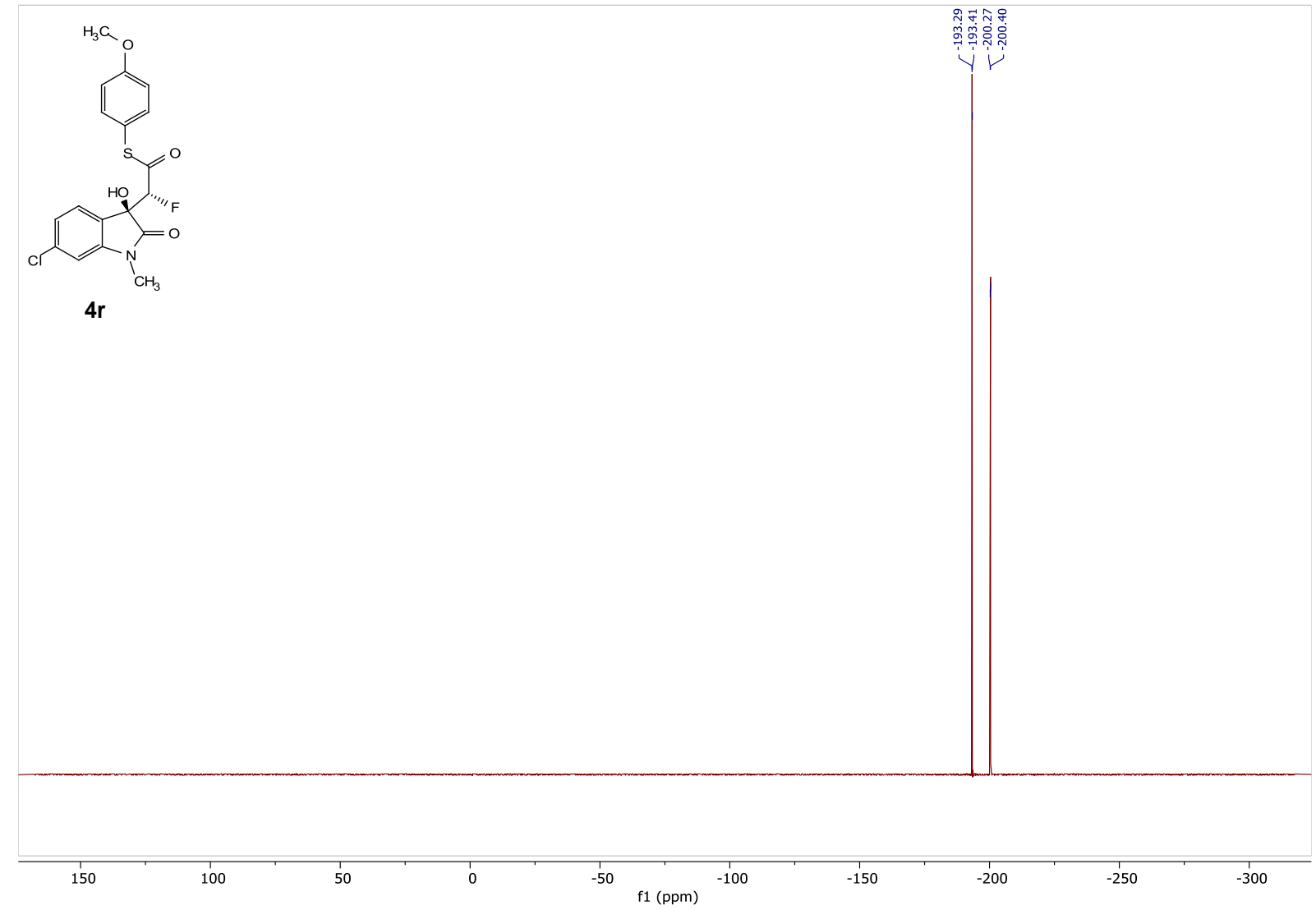

4s - ${ }^{1} \mathbf{H}$ NMR $\left(400 \mathrm{MHz}, \mathrm{C}_{6} \mathrm{D}_{6}\right)$

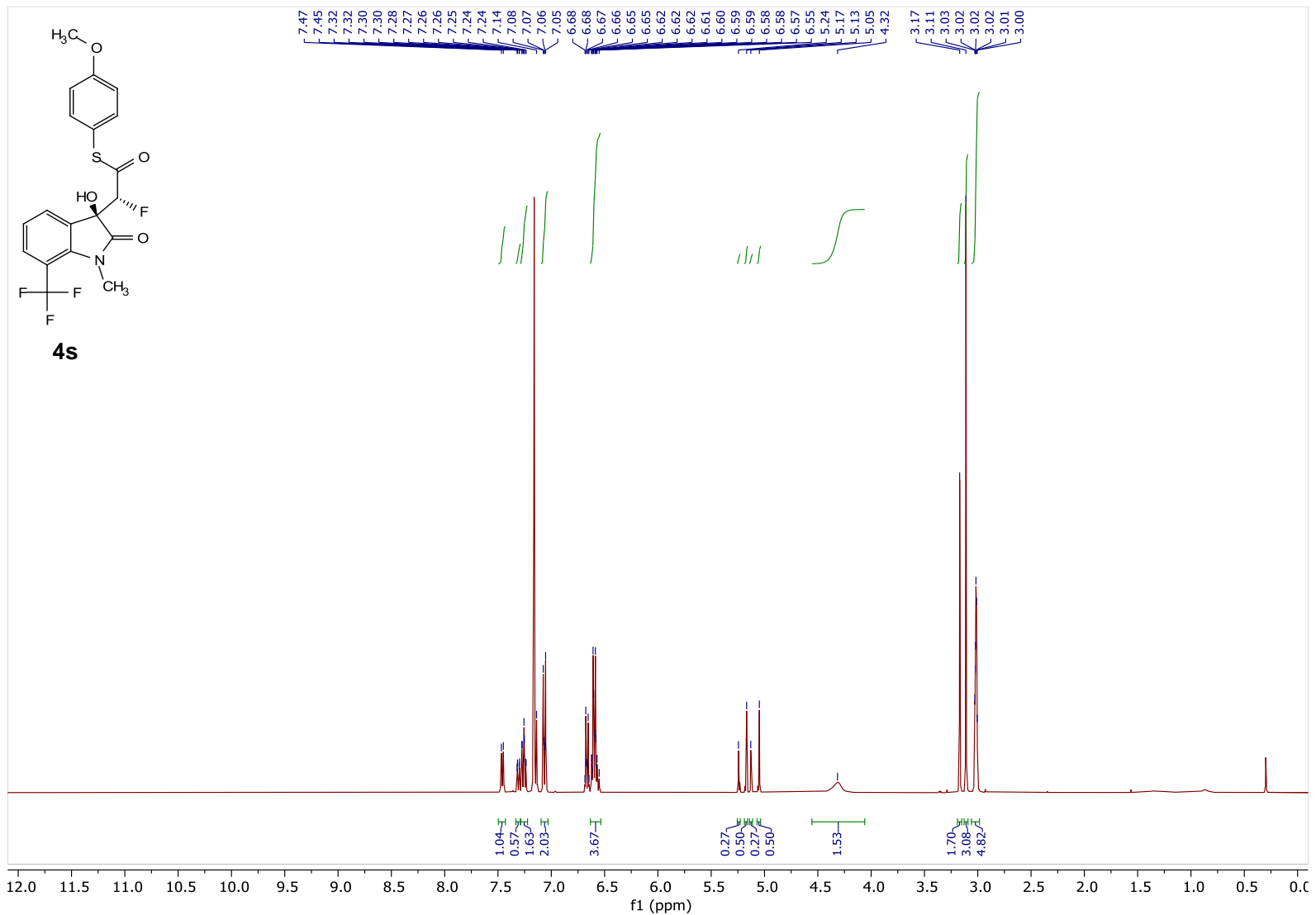


4s - ${ }^{13}$ C NMR (101 MHz, $\left.C_{6} D_{6}\right)$
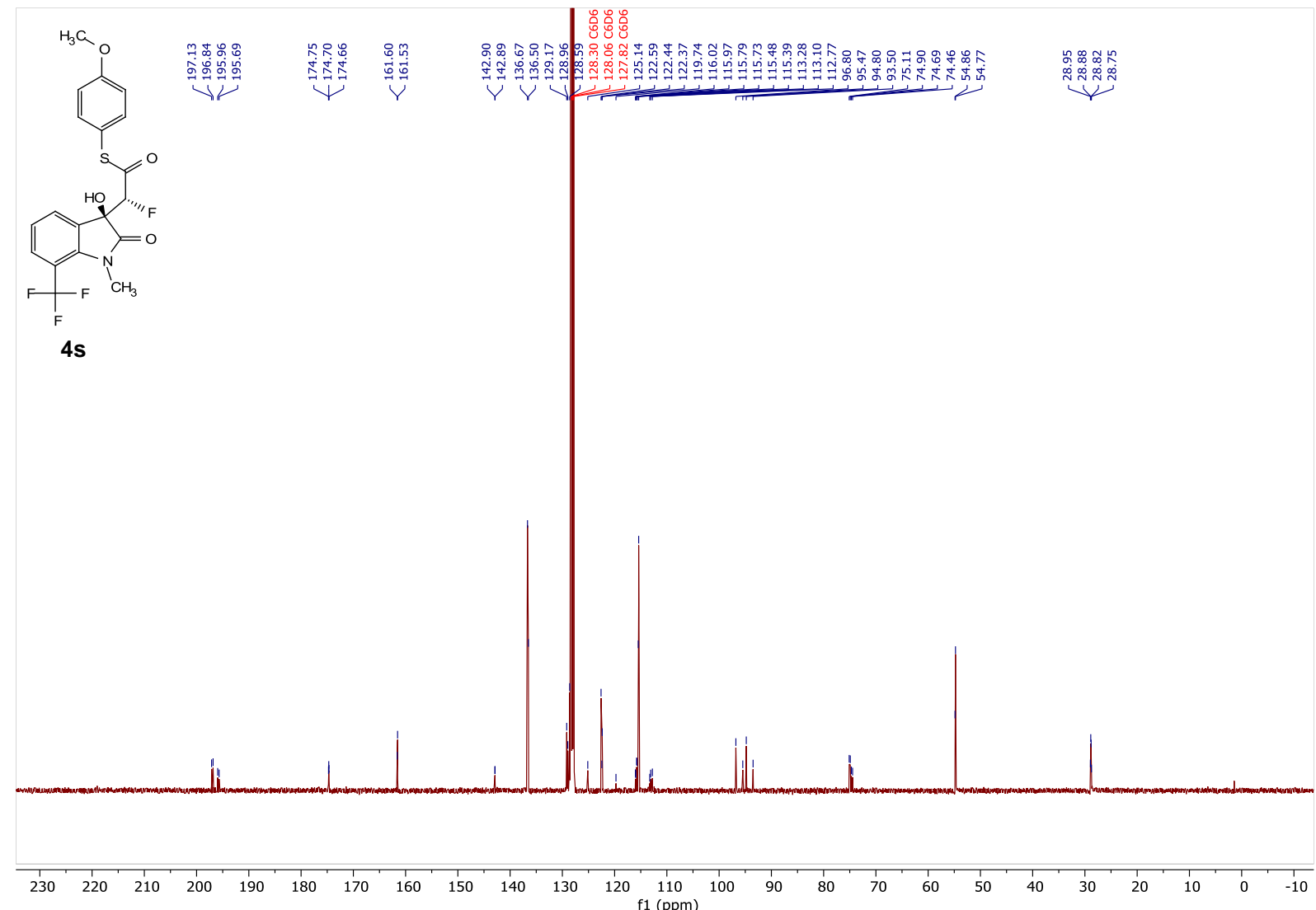

4s - ${ }^{19}$ F NMR (376 MHz, $\left.\mathrm{C}_{6} \mathrm{D}_{6}\right)$
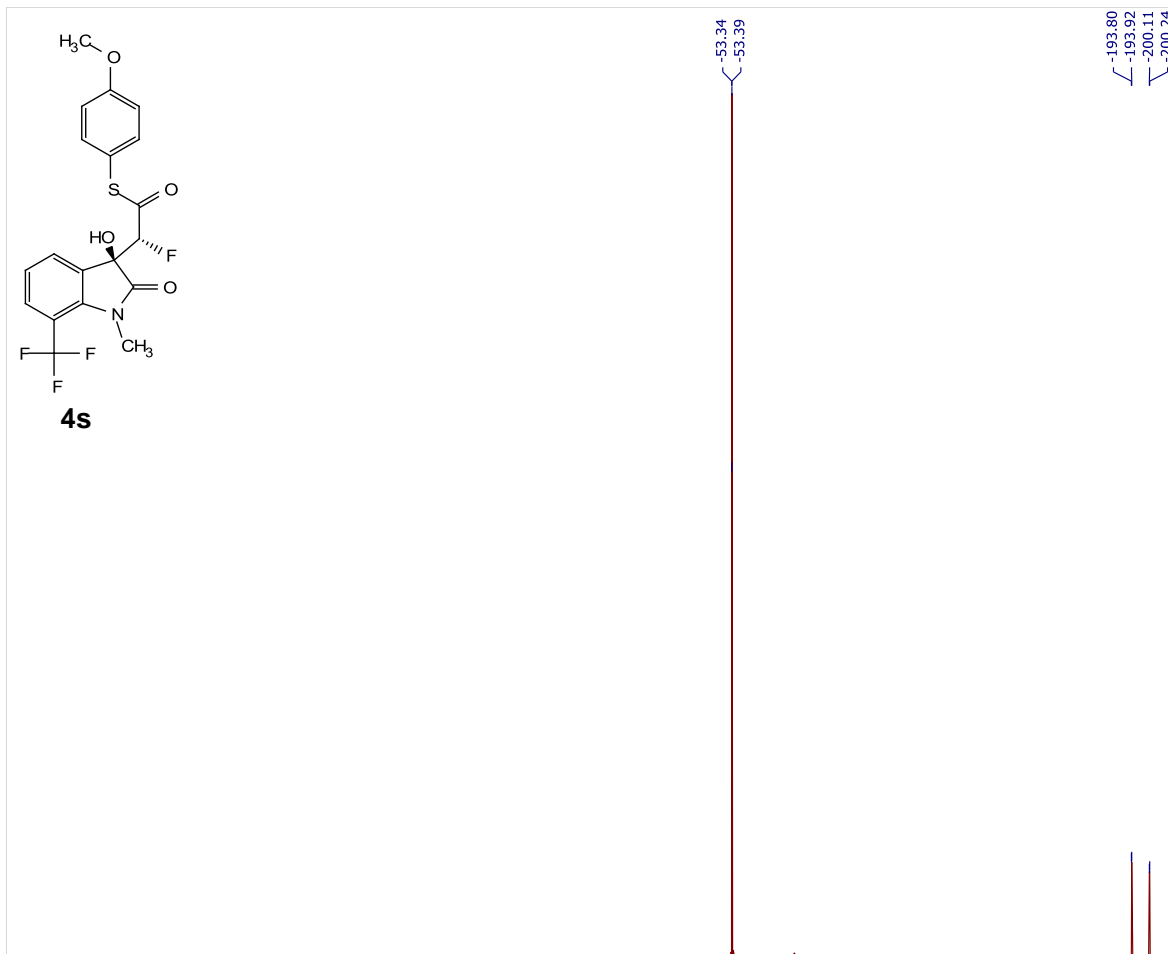

$: 00$

150

100

50

$-50 \mathrm{f1}(\mathrm{ppm})^{-100}$

$-150$

$-200$

$-250$

$-300$ 


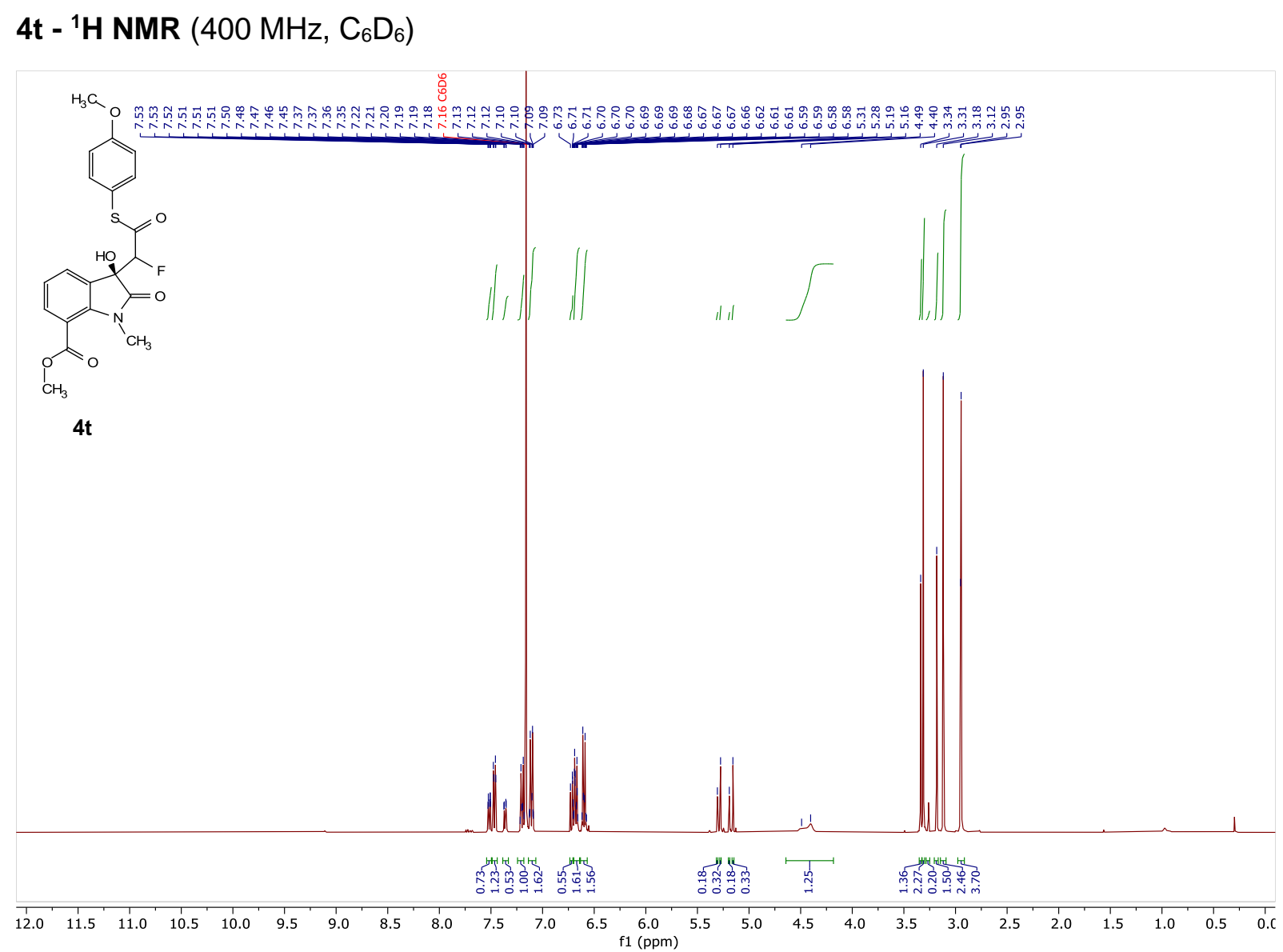

4t $-{ }^{13} \mathrm{C}$ NMR $\left(101 \mathrm{MHz}, \mathrm{C}_{6} \mathrm{D}_{6}\right)$

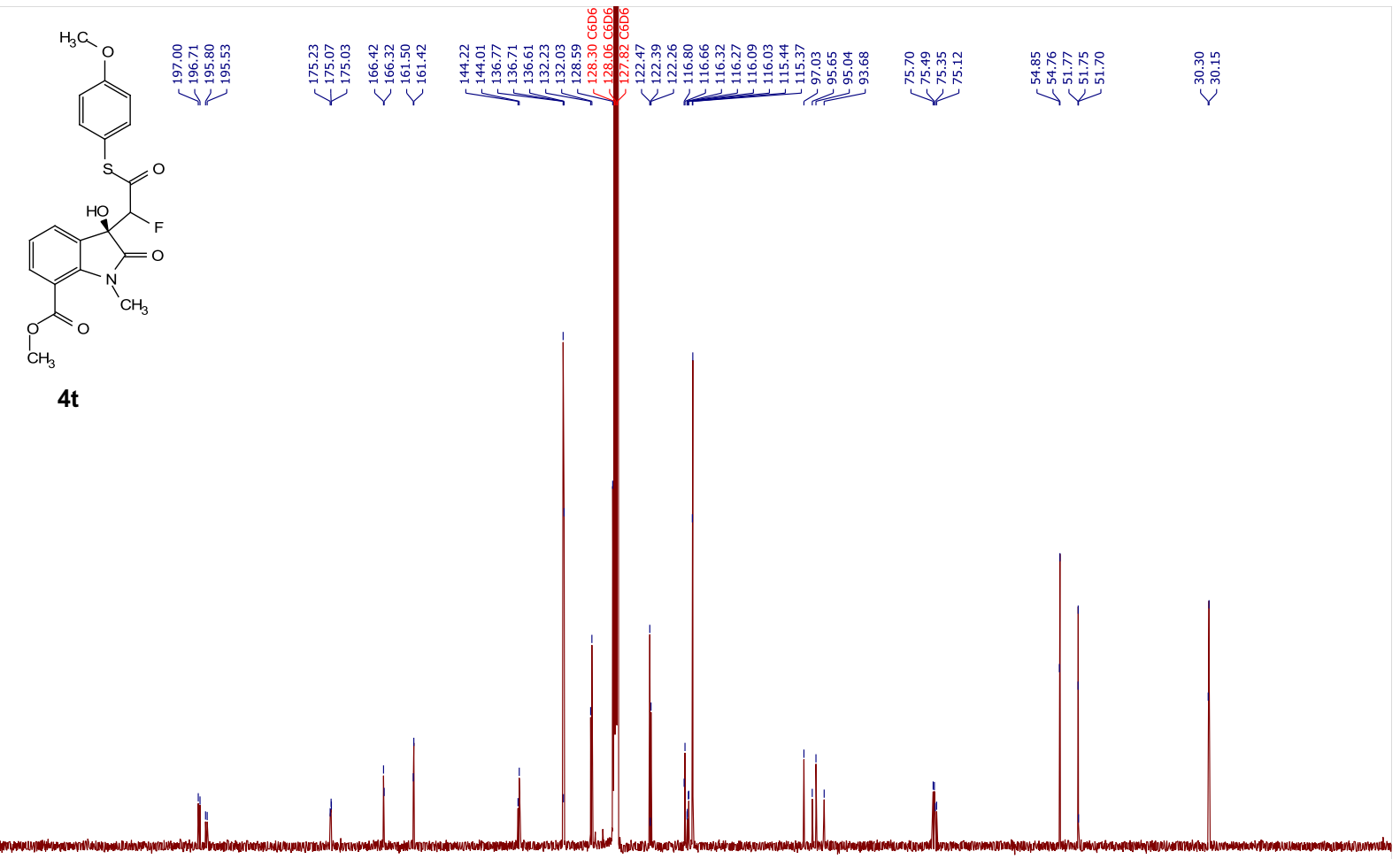

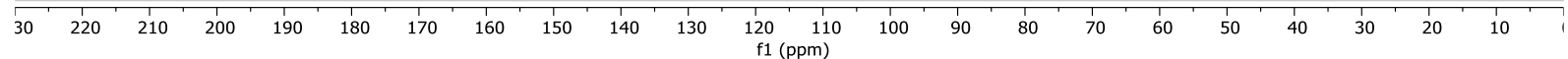


4t - ${ }^{19}$ F NMR (376 MHz, $\left.\mathrm{C}_{6} \mathrm{D}_{6}\right)$

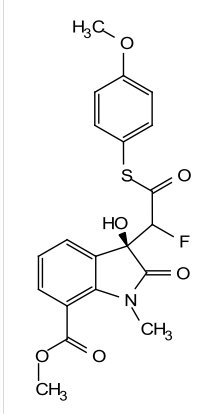

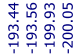

บriv

$4 t$

\begin{tabular}{|c|}
\hline 100 \\
\hline
\end{tabular}

\section{4u - ${ }^{1} \mathbf{H}$ NMR $\left(500 \mathrm{MHz}, \mathrm{C}_{6} \mathrm{D}_{6}\right)$}

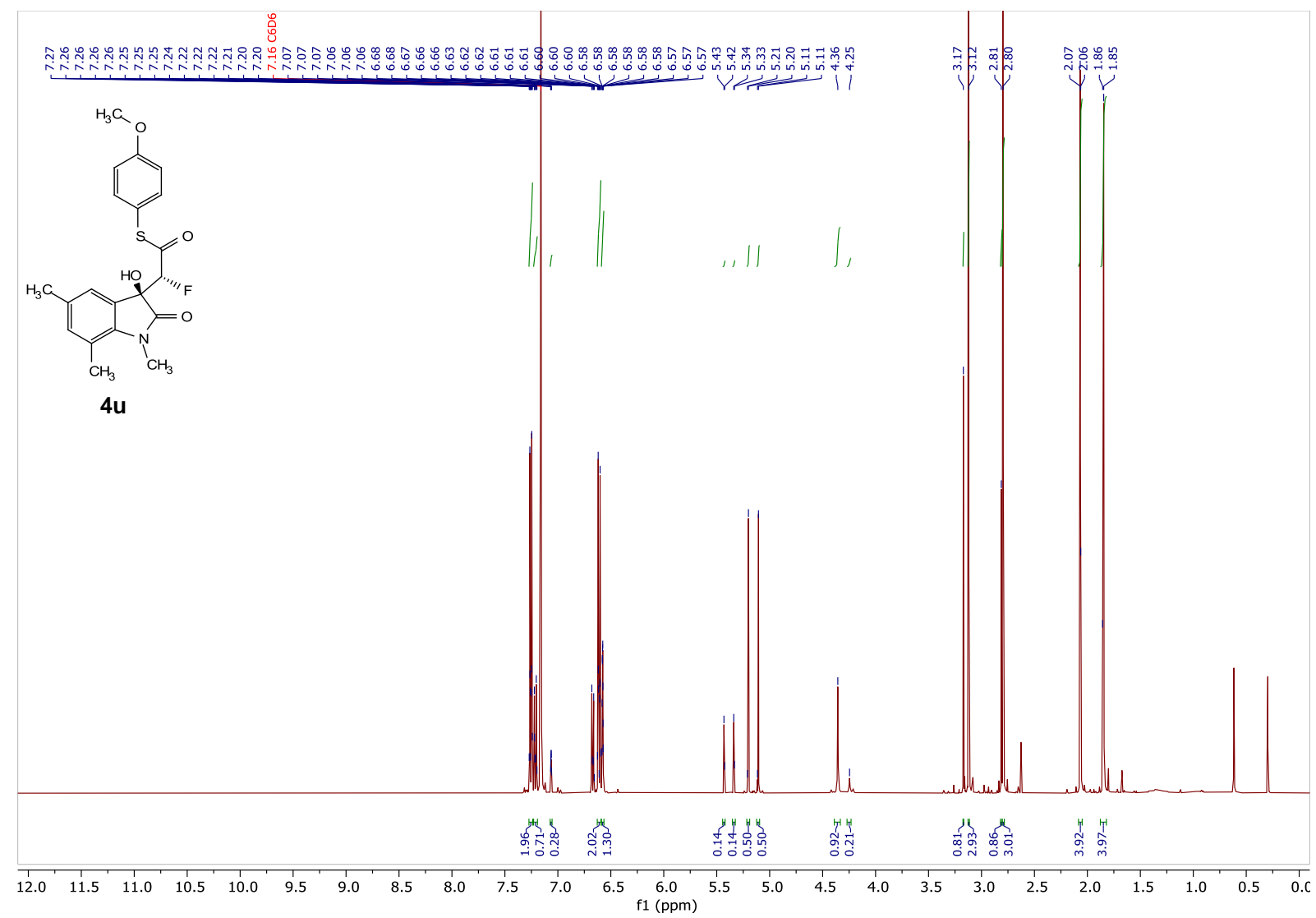


4u $-{ }^{13} \mathrm{C}$ NMR $\left(126 \mathrm{MHz}, \mathrm{C}_{6} \mathrm{D}_{6}\right)$
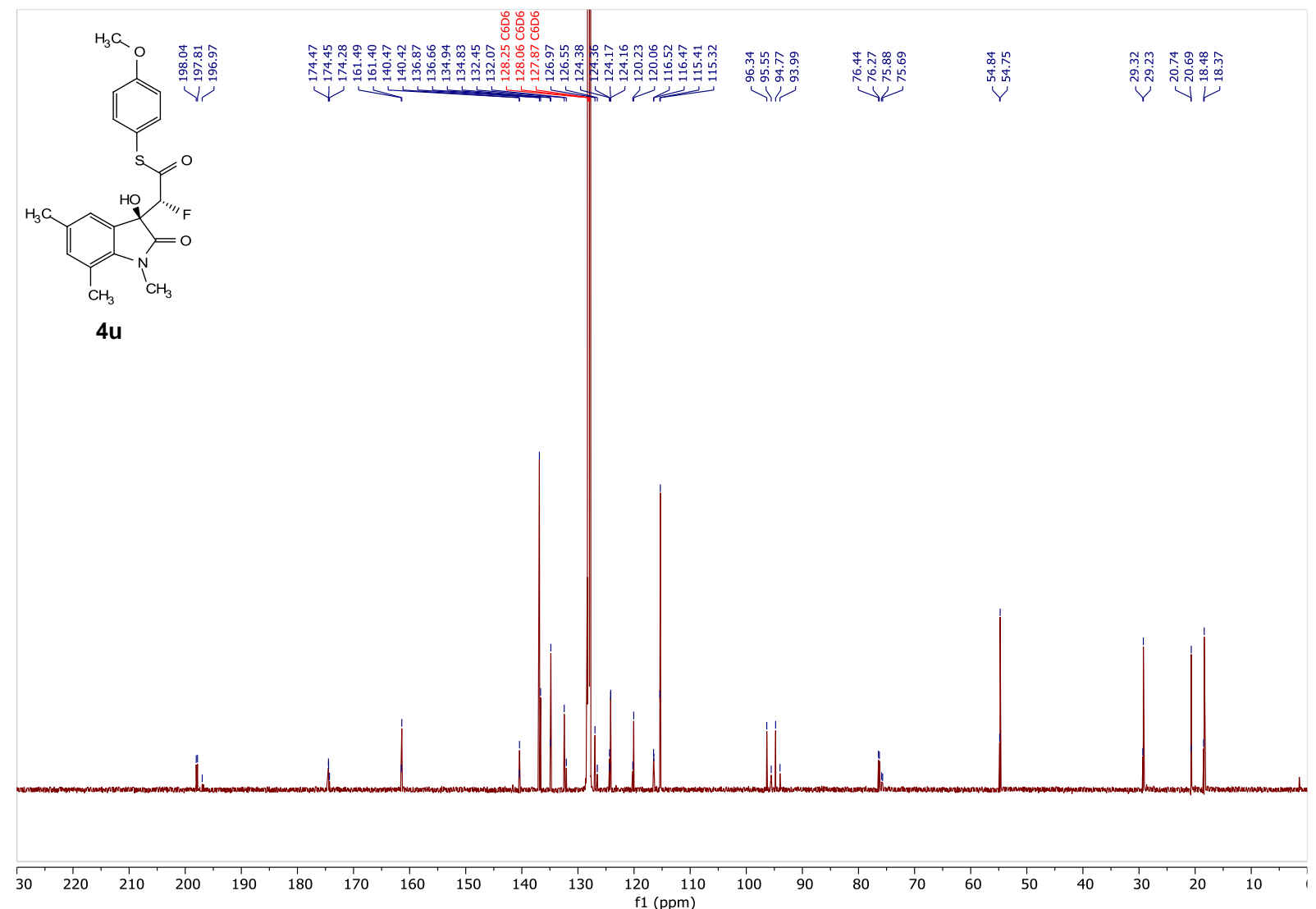

4u - ${ }^{19} \mathrm{~F}$ NMR $\left(471 \mathrm{MHz}, \mathrm{C}_{6} \mathrm{D}_{6}\right)$

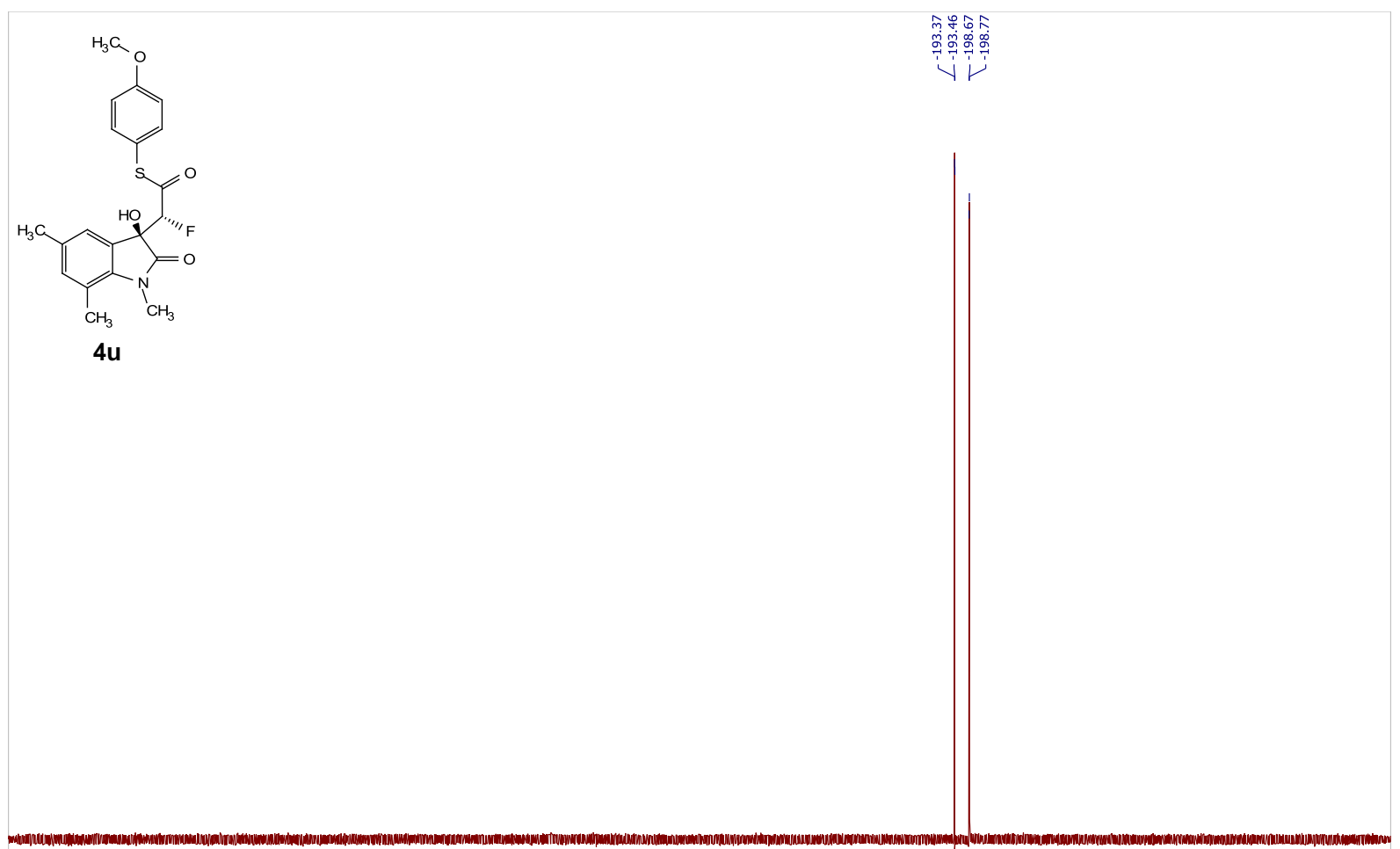


4v - ${ }^{1} \mathbf{H}$ NMR $\left(600 \mathrm{MHz}, \mathrm{C}_{6} \mathrm{D}_{6}\right)$

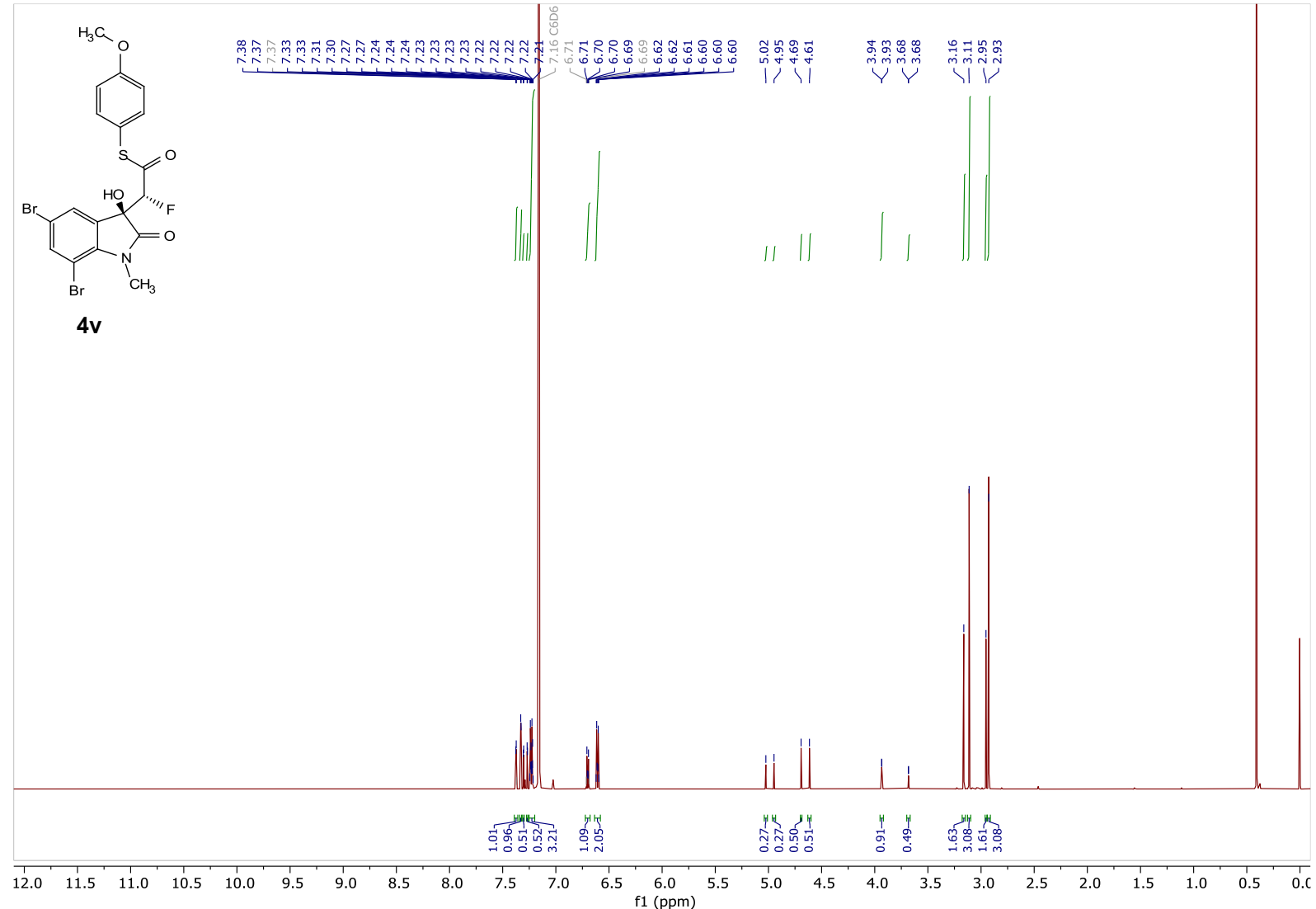

4v - ${ }^{13} \mathbf{C}$ NMR $\left(151 \mathrm{MHz}, \mathrm{C}_{6} \mathrm{D}_{6}\right)$

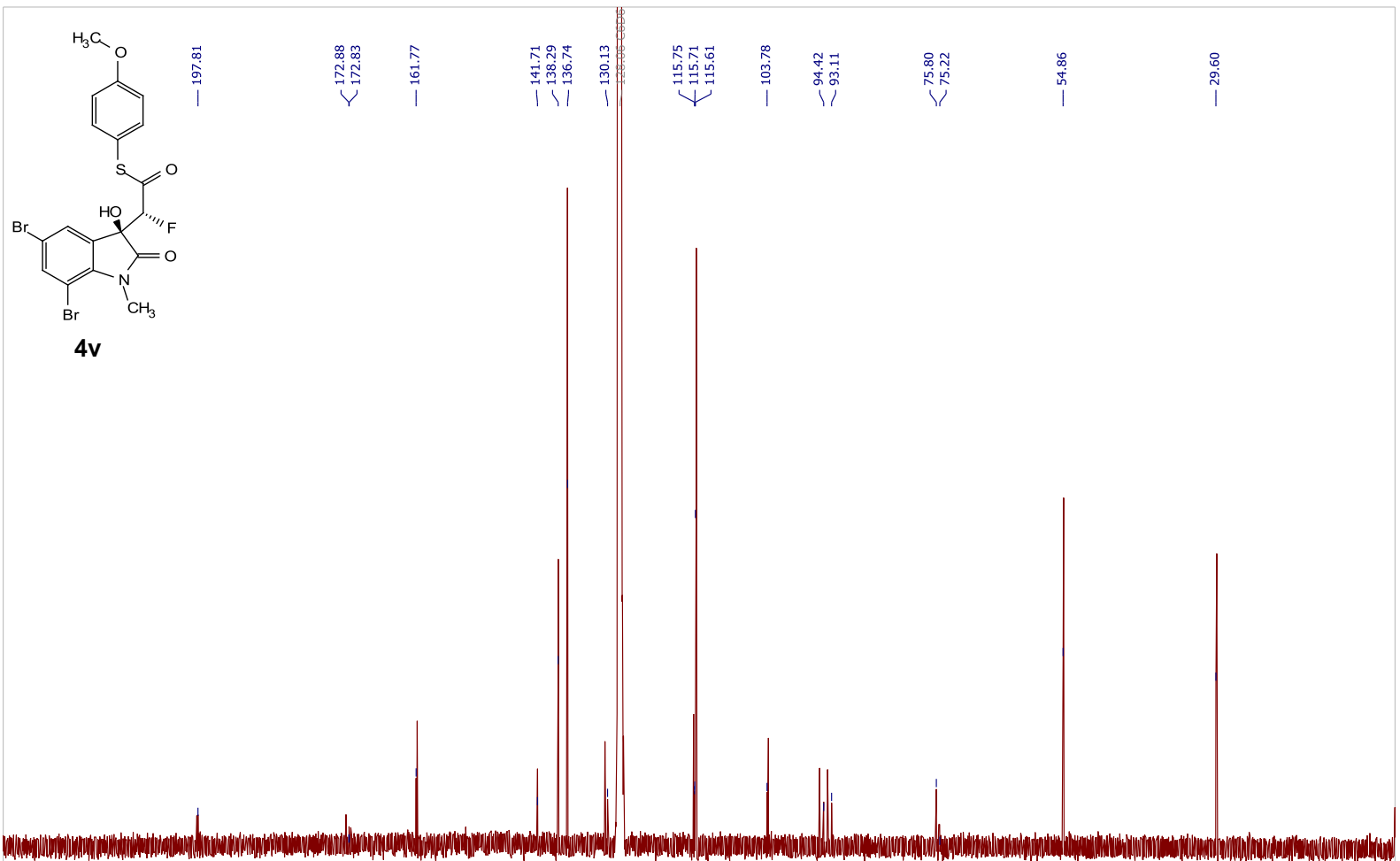

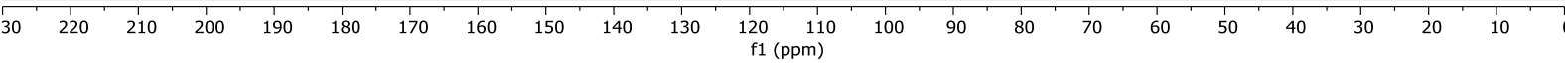


4v - ${ }^{19}$ F NMR $\left(470 \mathrm{MHz}, \mathrm{C}_{6} \mathrm{D}_{6}\right)$

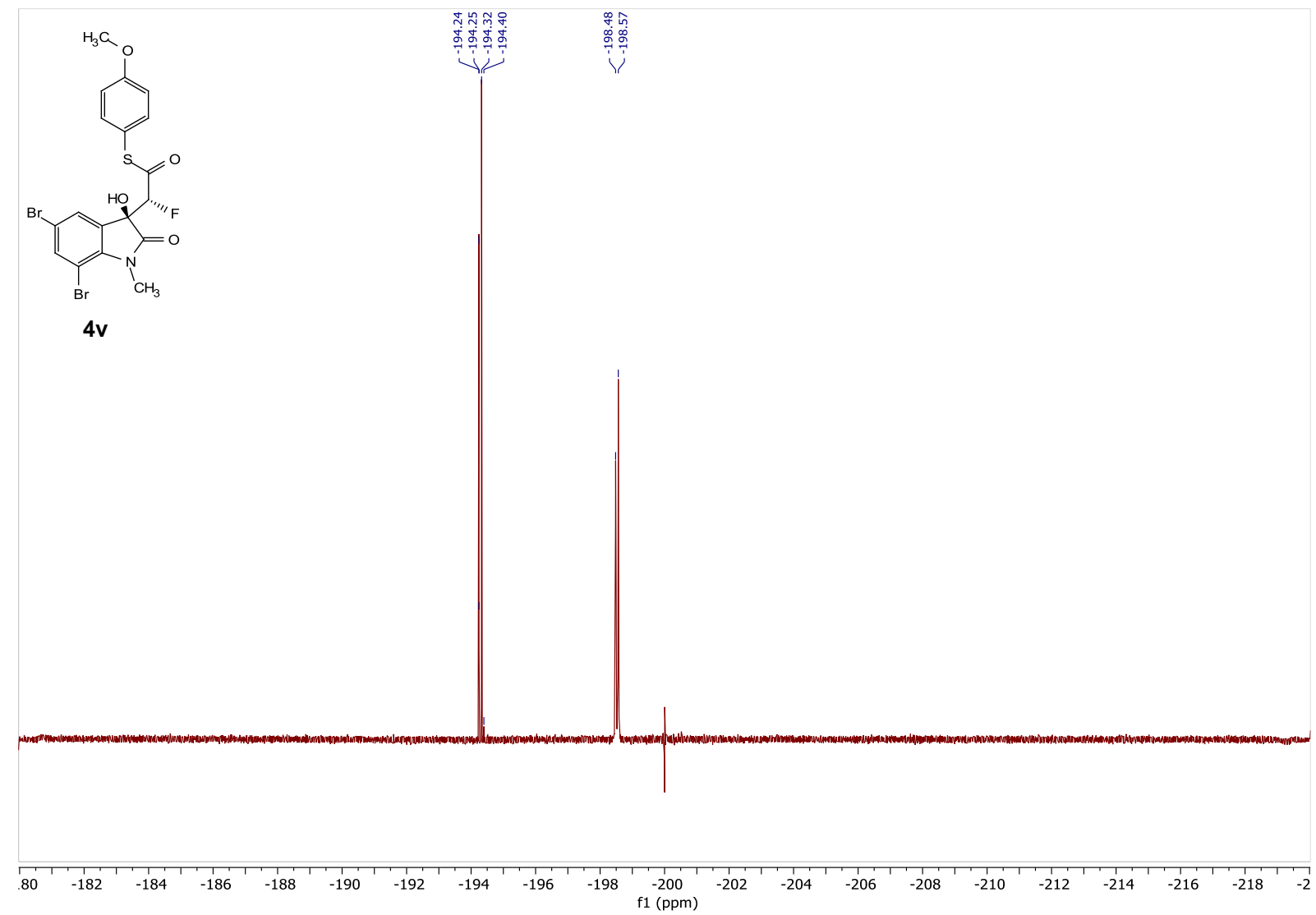

5 - ${ }^{1} \mathbf{H}$ NMR $\left(600 \mathrm{MHz}, \mathrm{CD}_{2} \mathrm{Cl}_{2}\right)$

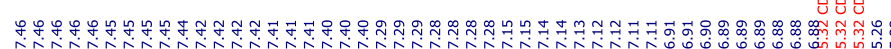

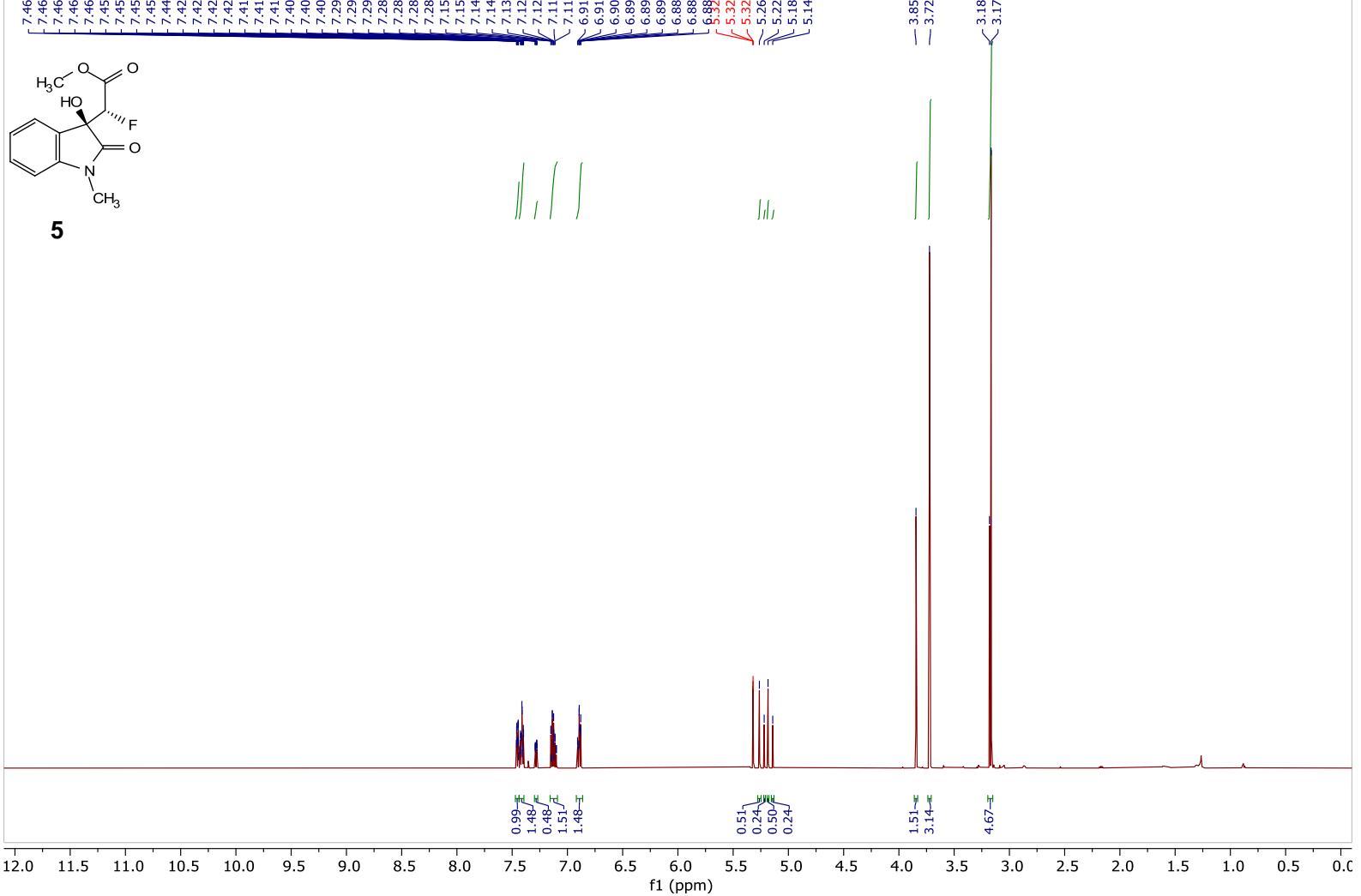


$5-{ }^{13} \mathbf{C}$ NMR $\left(151 \mathrm{MHz}, \mathrm{CD}_{2} \mathrm{Cl}_{2}\right)$

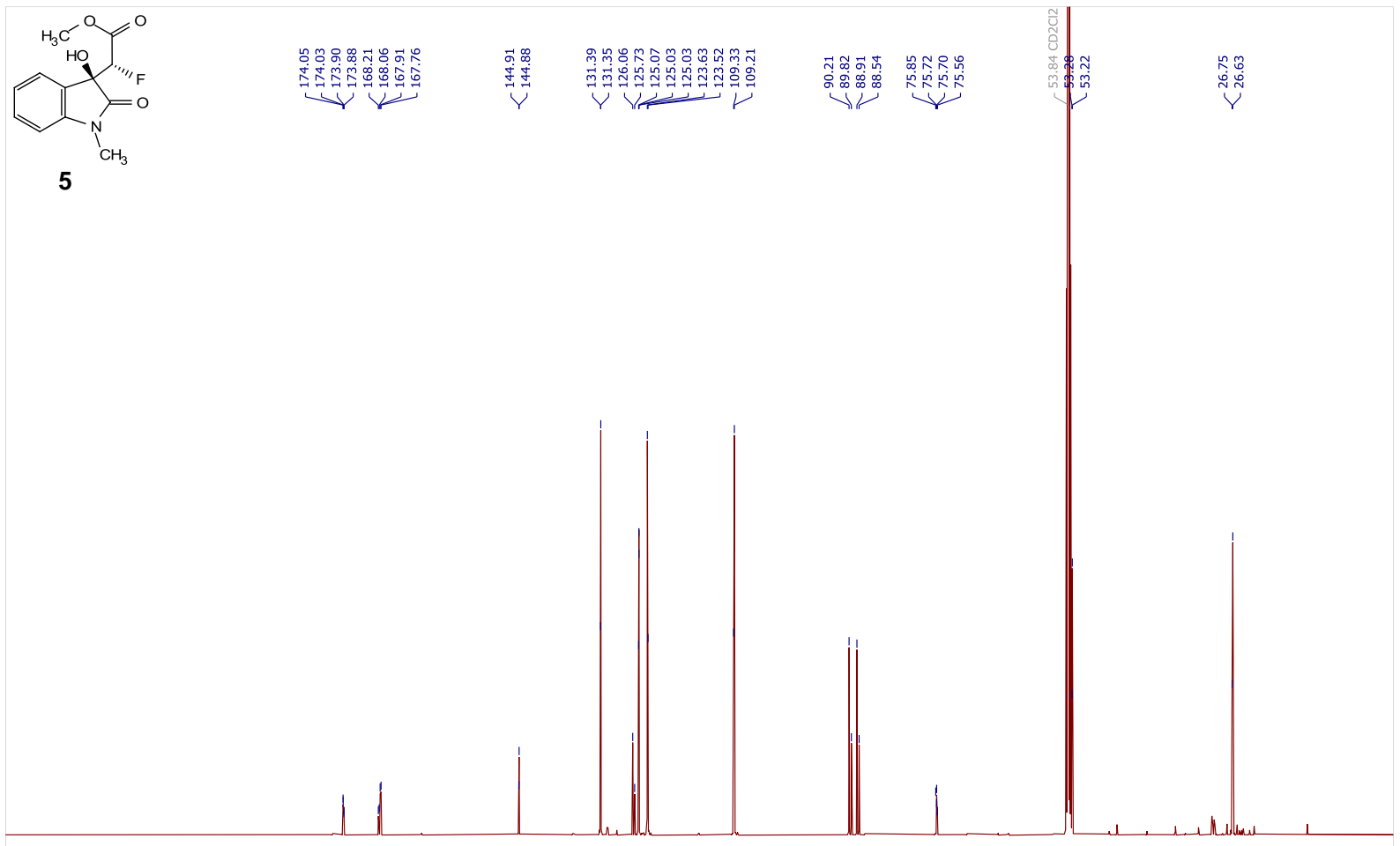

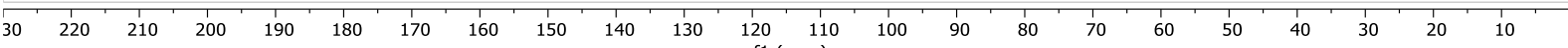

6 - ${ }^{1} \mathrm{H}$ NMR $\left(600 \mathrm{MHz}, \mathrm{CD}_{2} \mathrm{Cl}_{2}\right)$

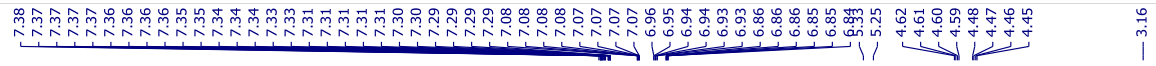

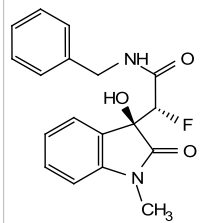

6
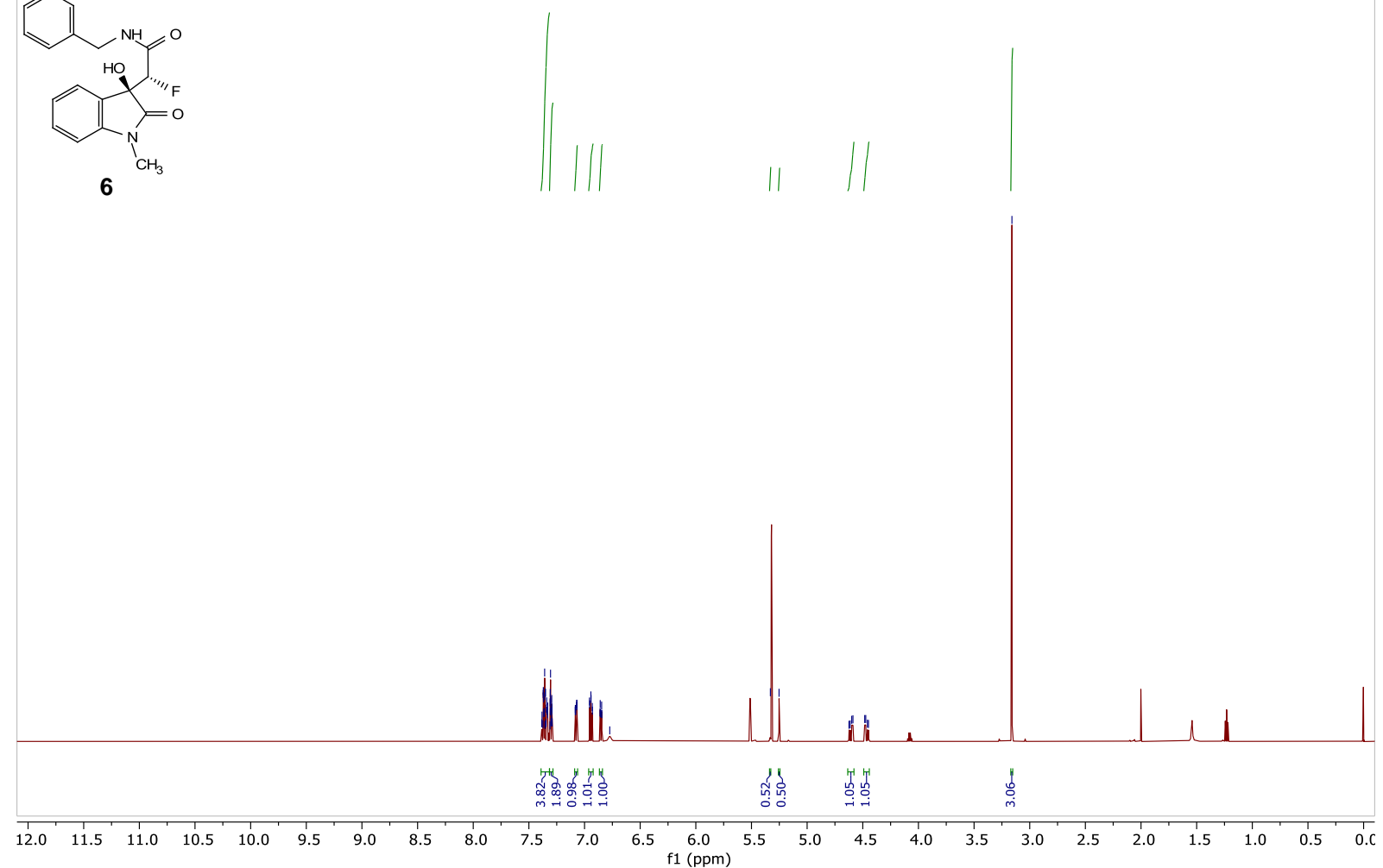
$6-{ }^{13} \mathrm{C}$ NMR (151 MHz, $\left.\mathrm{CD}_{2} \mathrm{Cl}_{2}\right)$
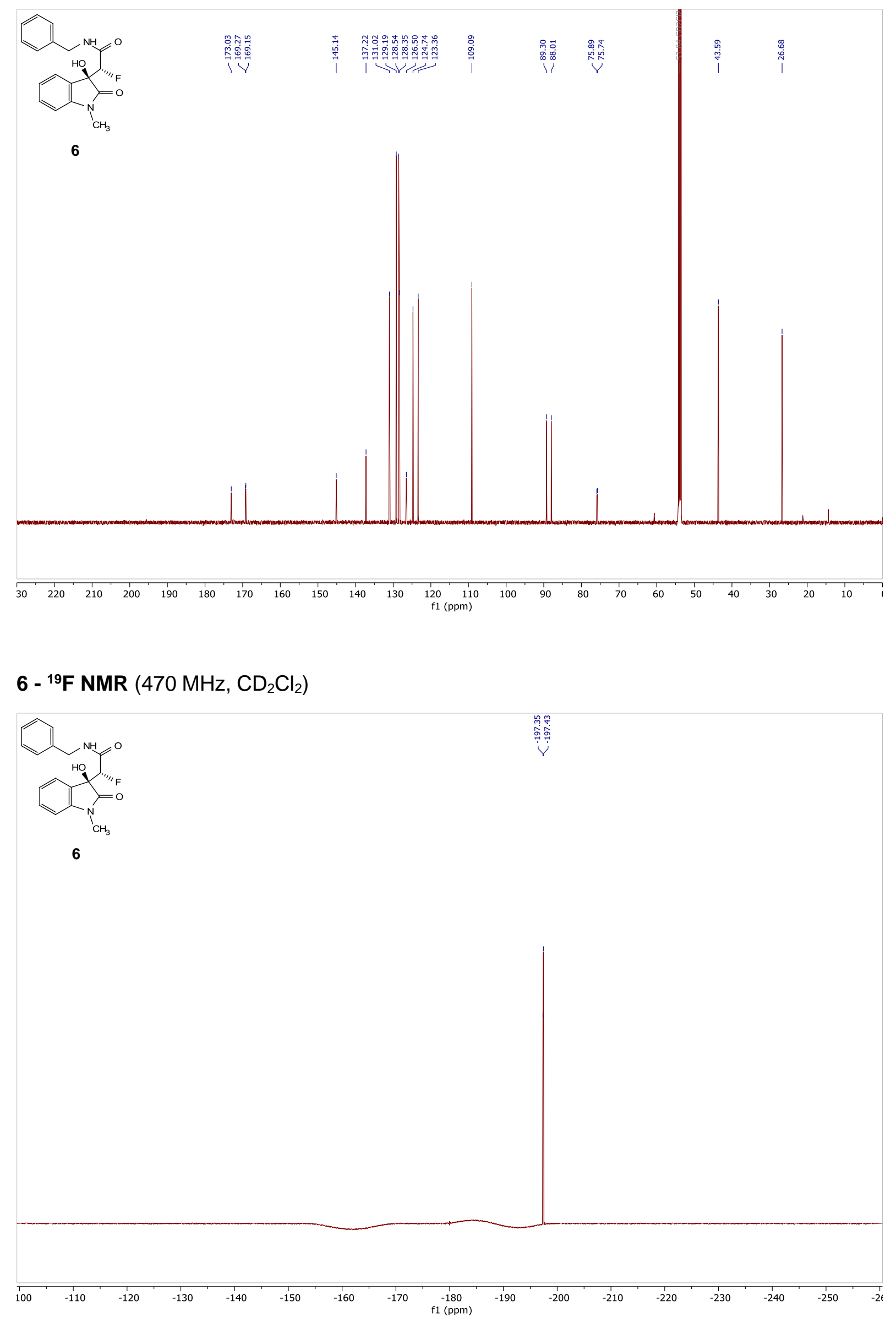
6 - Minor Diastereoisomer - ${ }^{1} \mathrm{H}$ NMR $\left(600 \mathrm{MHz}, \mathrm{CD}_{2} \mathrm{Cl}_{2}\right)$

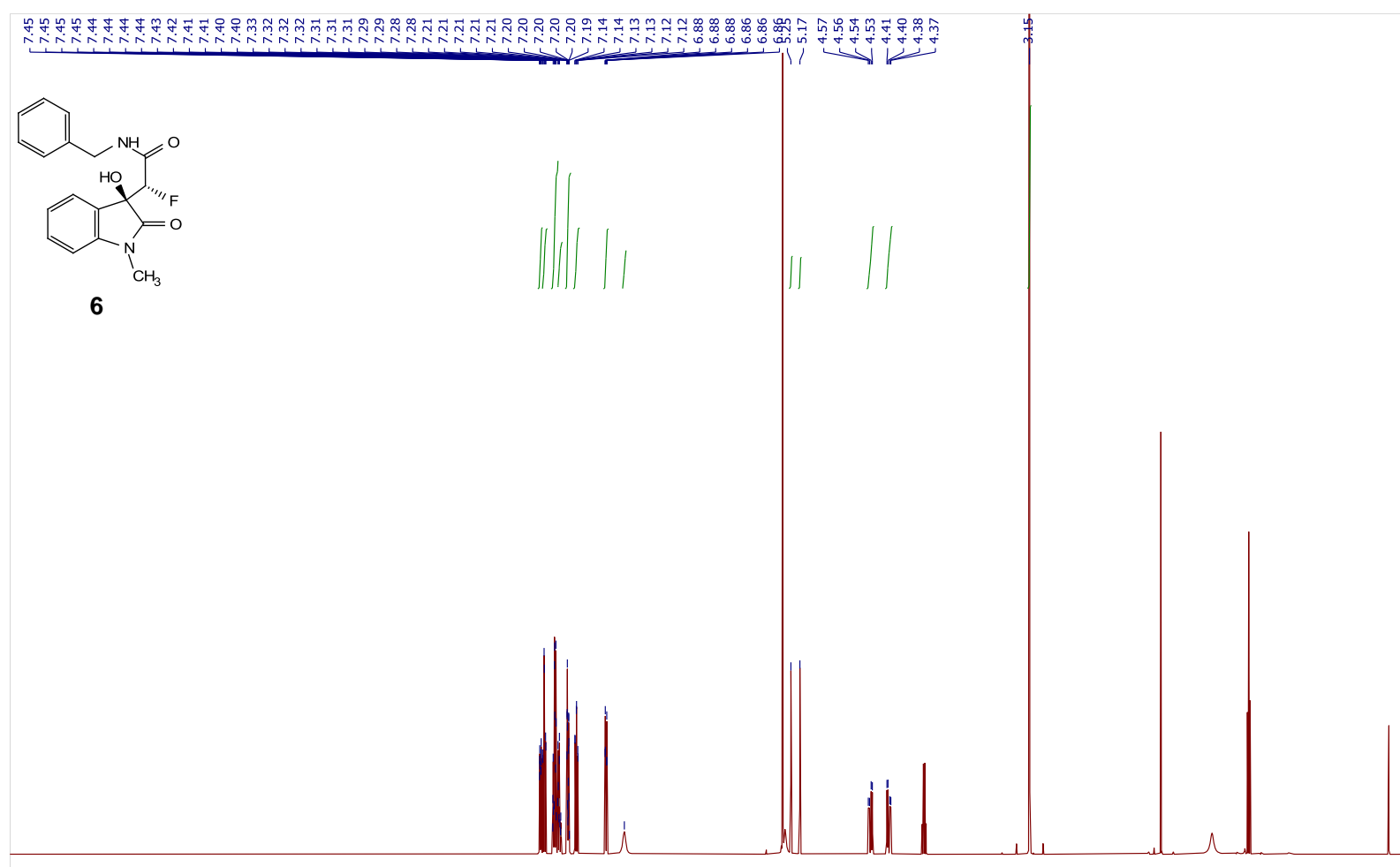

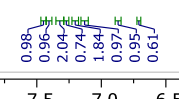

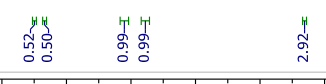

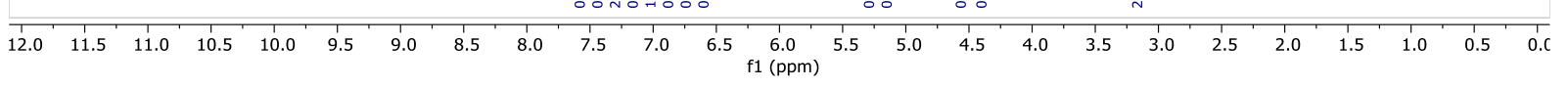

$6-{ }^{13} \mathrm{C}$ NMR $\left(151 \mathrm{MHz}, \mathrm{CD}_{2} \mathrm{Cl}_{2}\right)$

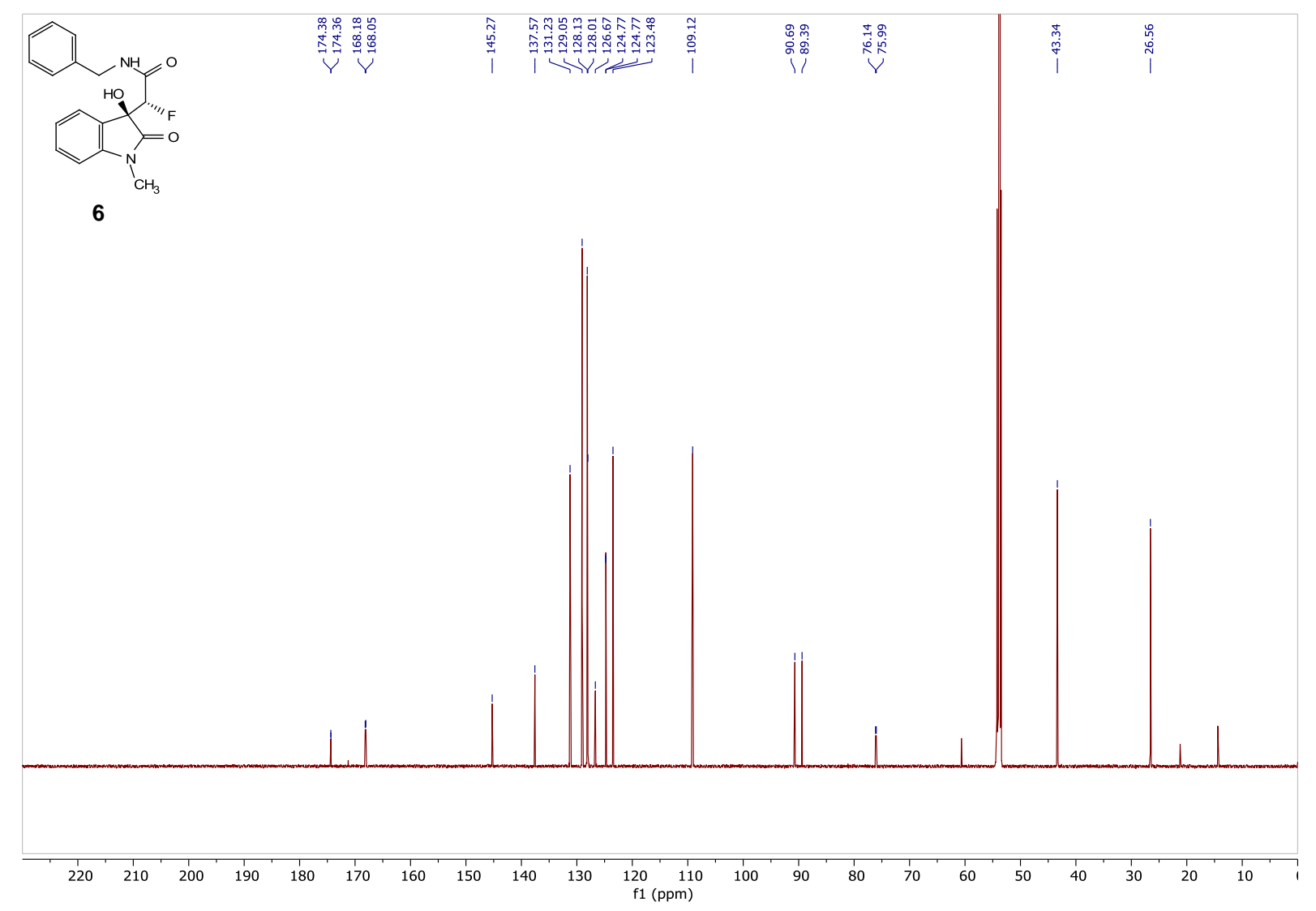


$6-{ }^{19} \mathrm{~F}$ NMR $\left(470 \mathrm{MHz}, \mathrm{CD}_{2} \mathrm{Cl}_{2}\right)$

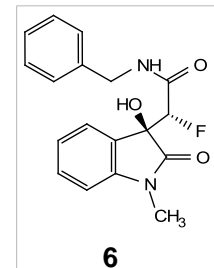

\section{กิัยำ}

6

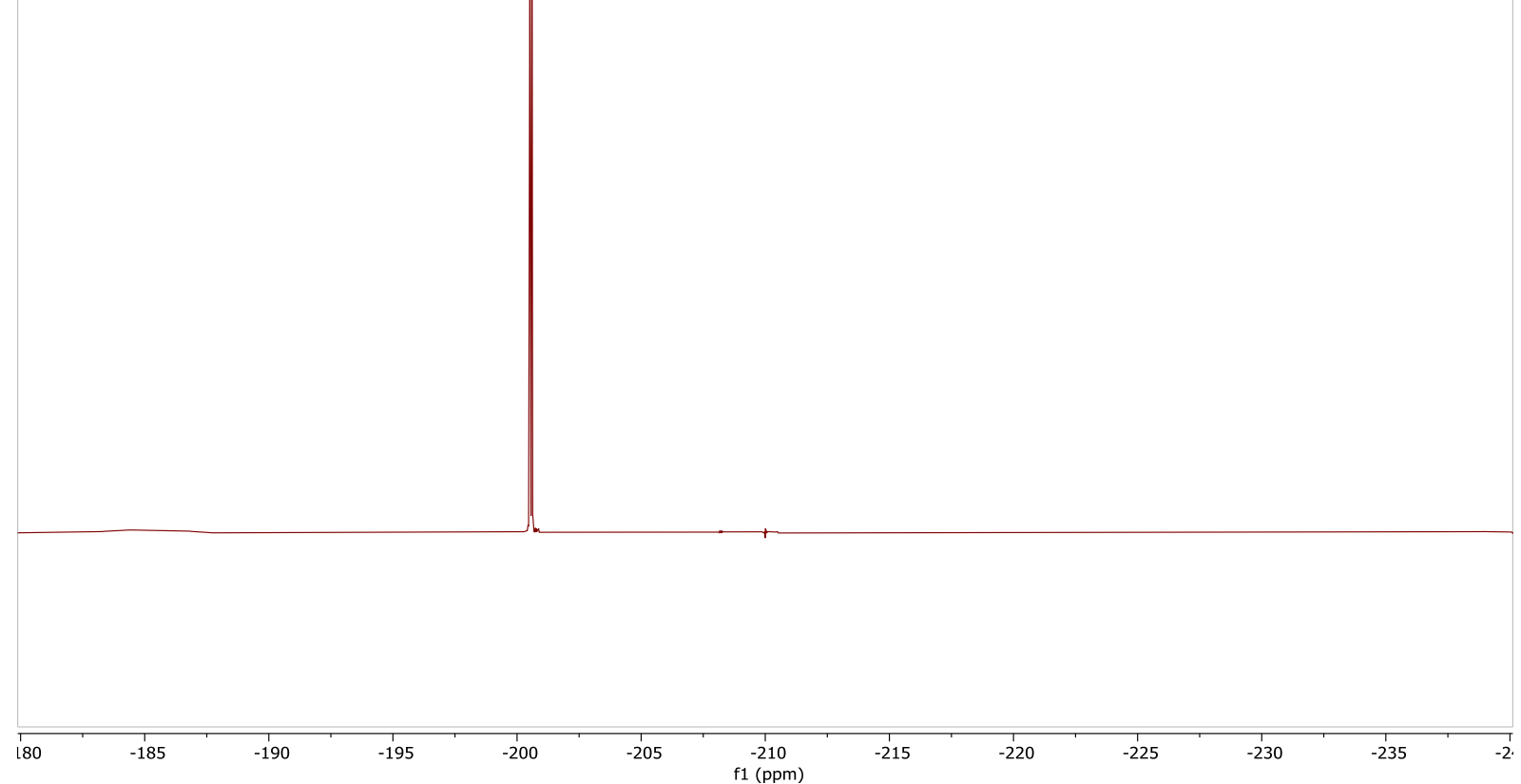

7 - ${ }^{1} \mathbf{H}$ NMR $\left(500 \mathrm{MHz}, \mathrm{CD}_{2} \mathrm{Cl}_{2}\right)$

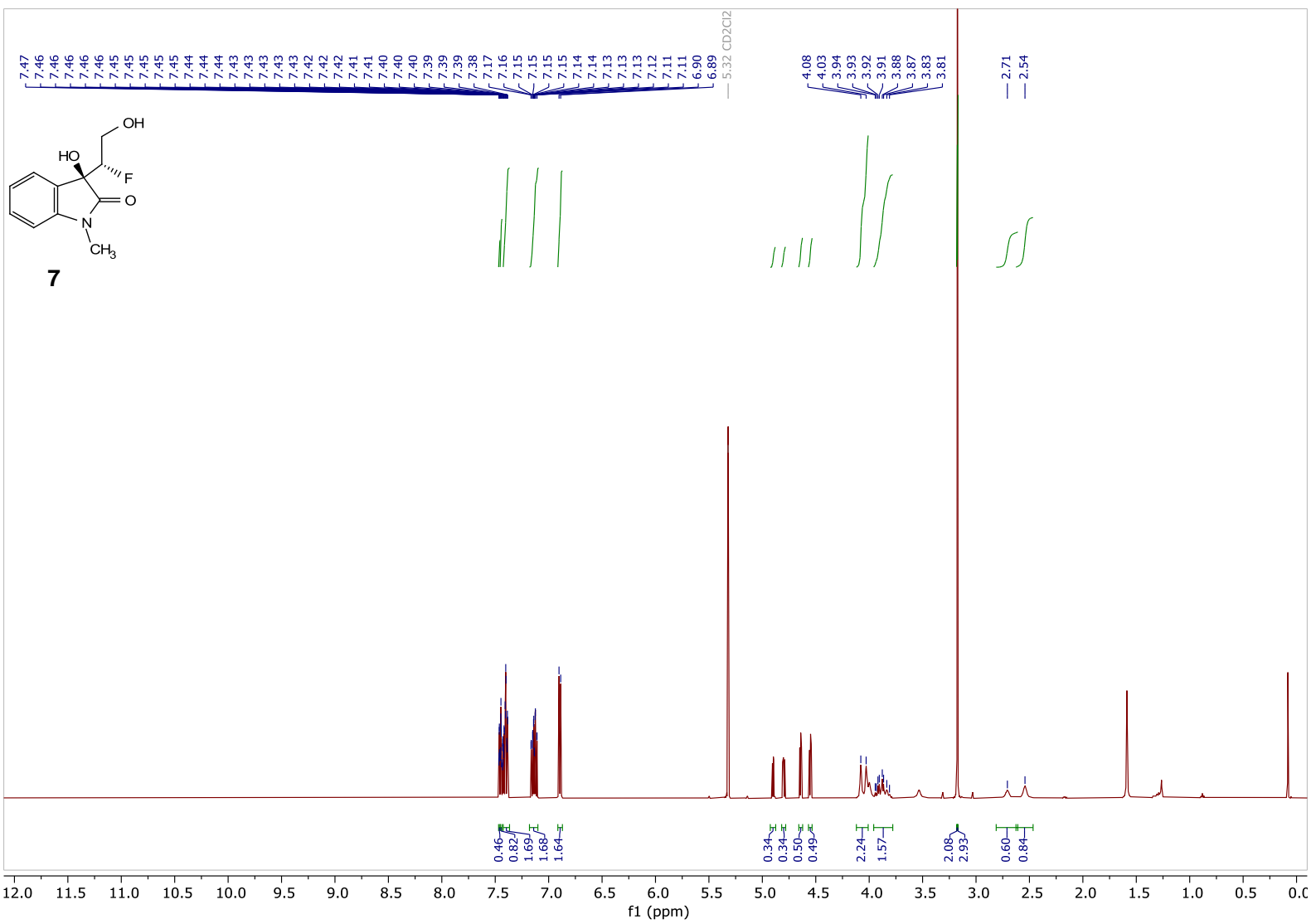


$7={ }^{13} \mathrm{C}$ NMR $\left(126 \mathrm{MHz}, \mathrm{CD}_{2} \mathrm{Cl}_{2}\right)$

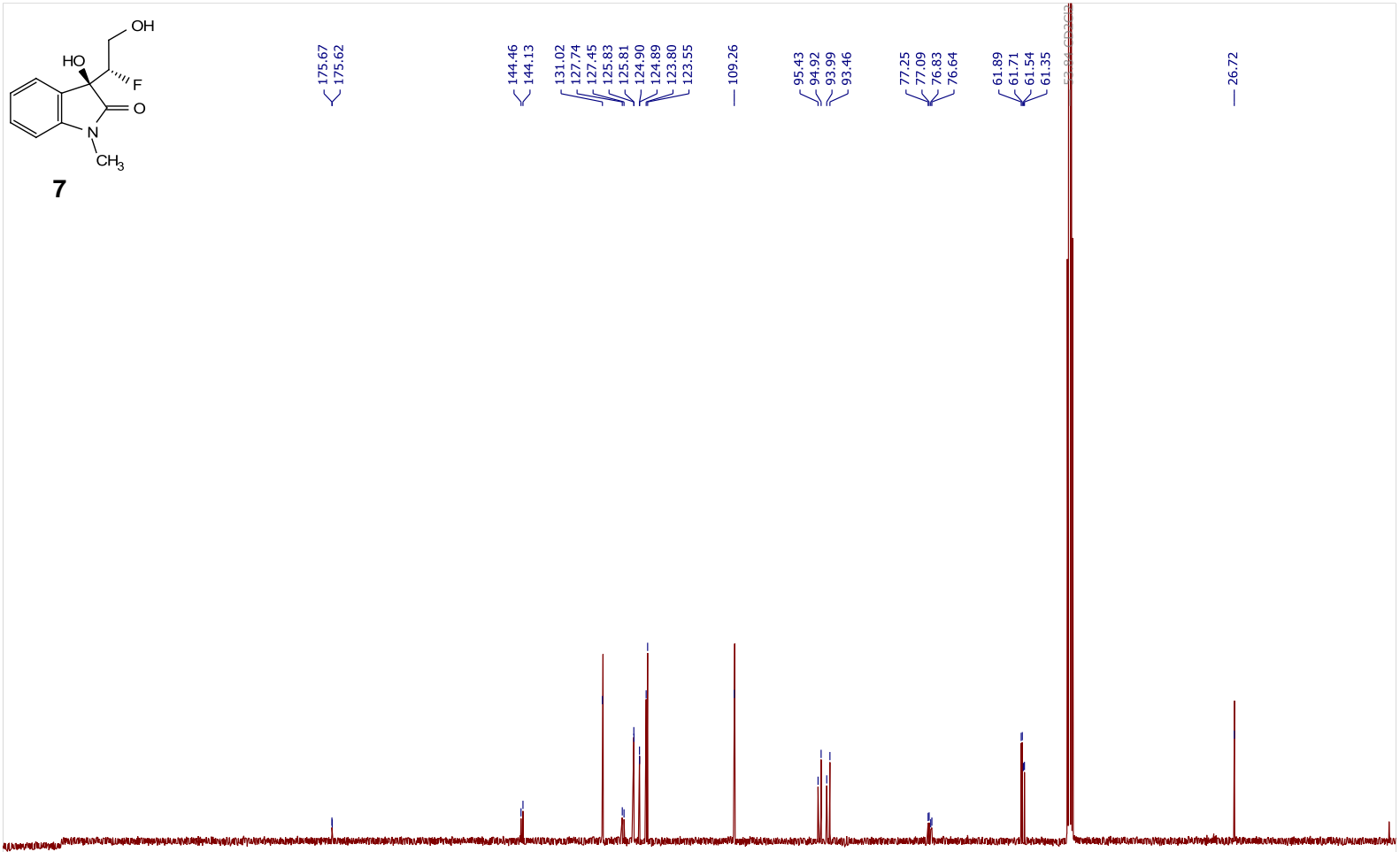

\begin{tabular}{|lllllllllllllllllllllllllll}
\hline 30 & 220 & 210 & 200 & 190 & 180 & 170 & 160 & 150 & 140 & 130 & 120 & 110 & 100 & 90 & 80 & 70 & 60 & 50 & 40 & 30 & 20 & 10 & 10
\end{tabular}

7 - ${ }^{19} \mathrm{~F}$ NMR $\left(471 \mathrm{MHz}, \mathrm{CD}_{2} \mathrm{Cl}_{2}\right)$

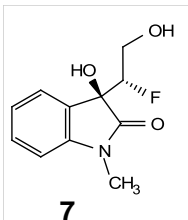


8 - ${ }^{1} \mathrm{H}$ NMR $\left(500 \mathrm{MHz}, \mathrm{C}_{6} \mathrm{D}_{6}\right)$

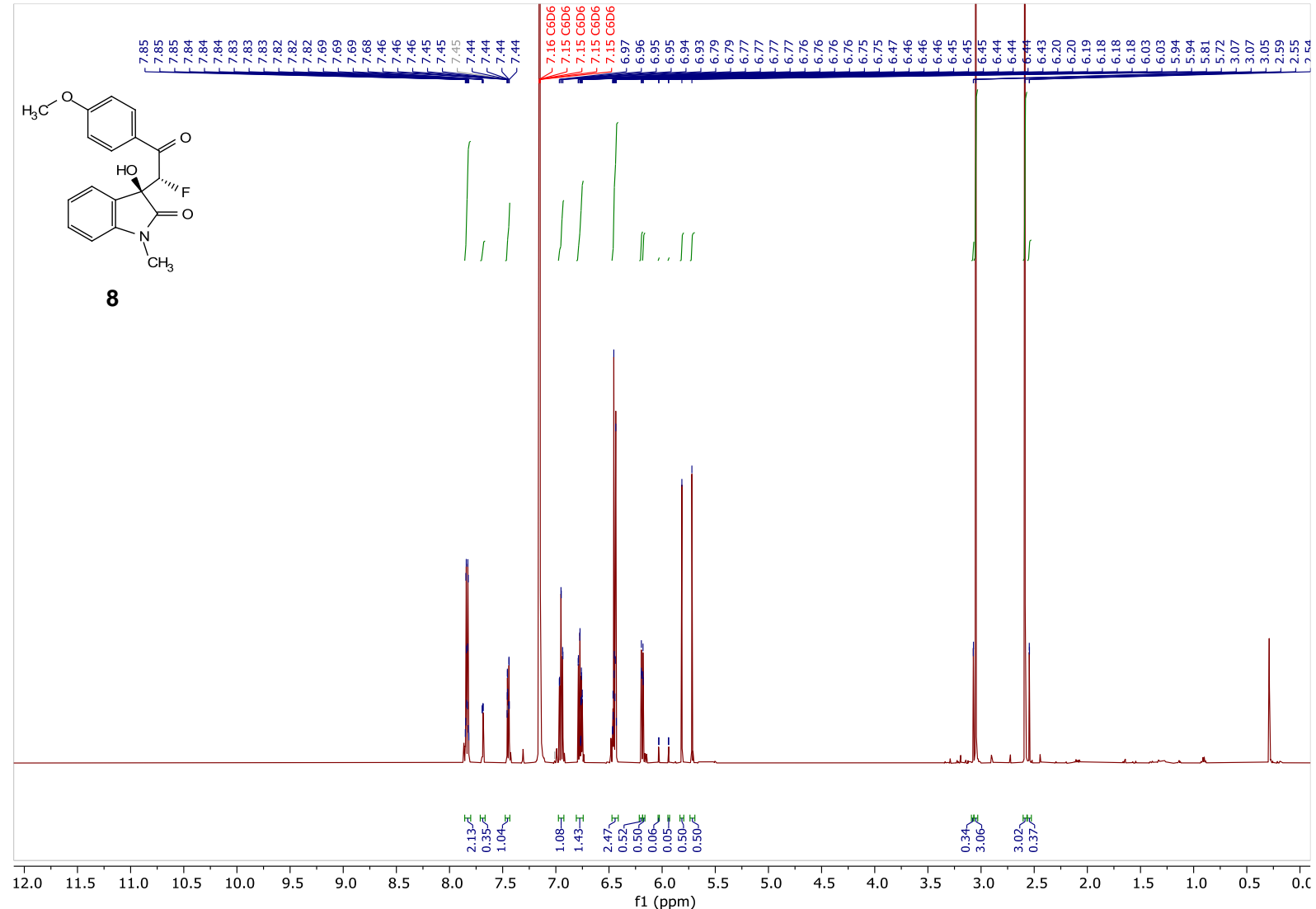

$8-{ }^{13} \mathrm{C}$ NMR $\left(126 \mathrm{MHz}, \mathrm{C}_{6} \mathrm{D}_{6}\right)$
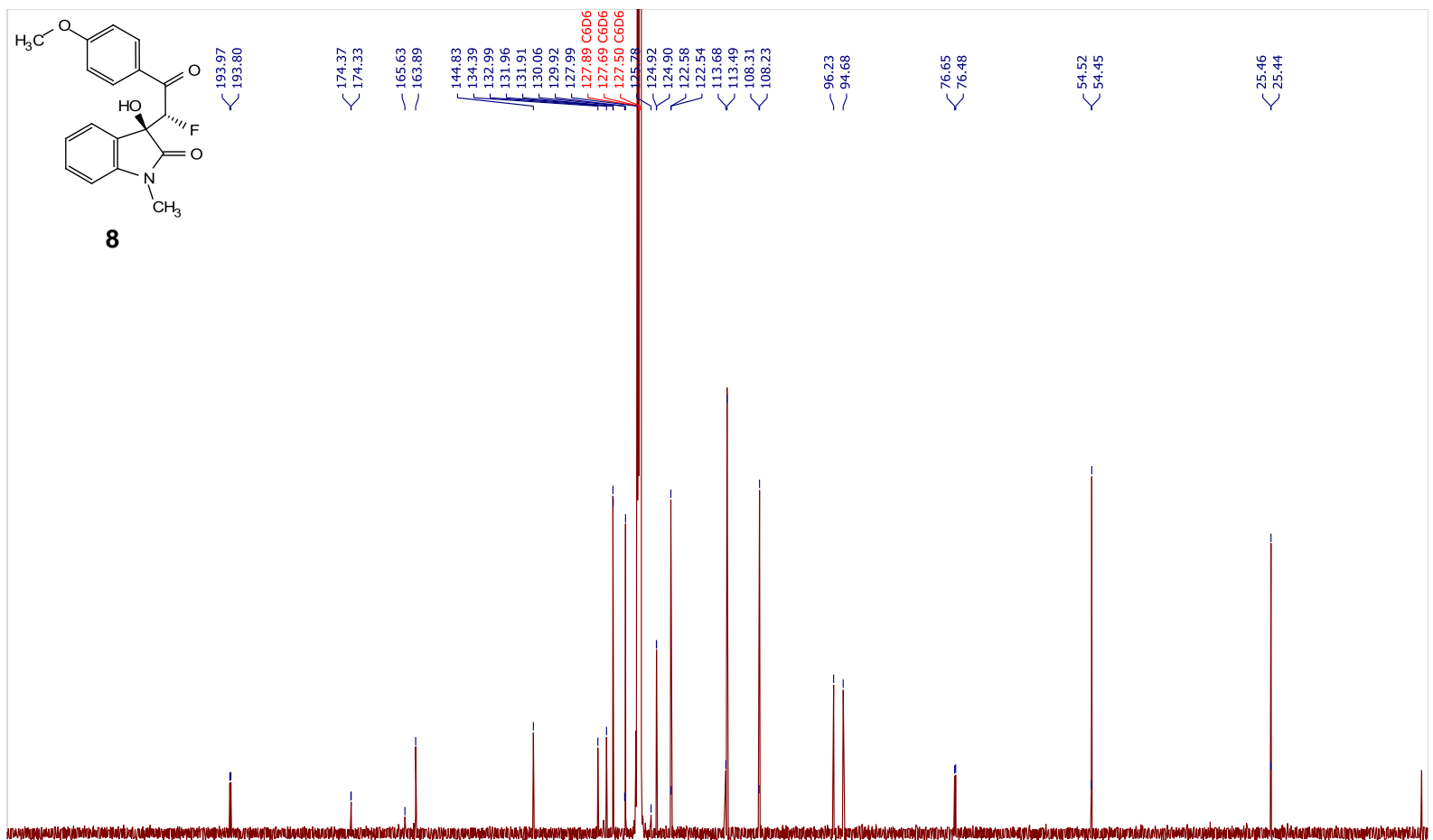

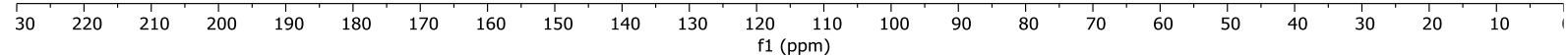


8 - ${ }^{19}$ F NMR $\left(471 \mathrm{MHz}, \mathrm{C}_{6} \mathrm{D}_{6}\right)$

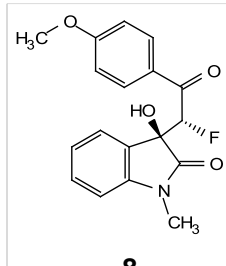

8

F

ن

$\mathrm{CH}_{3}$

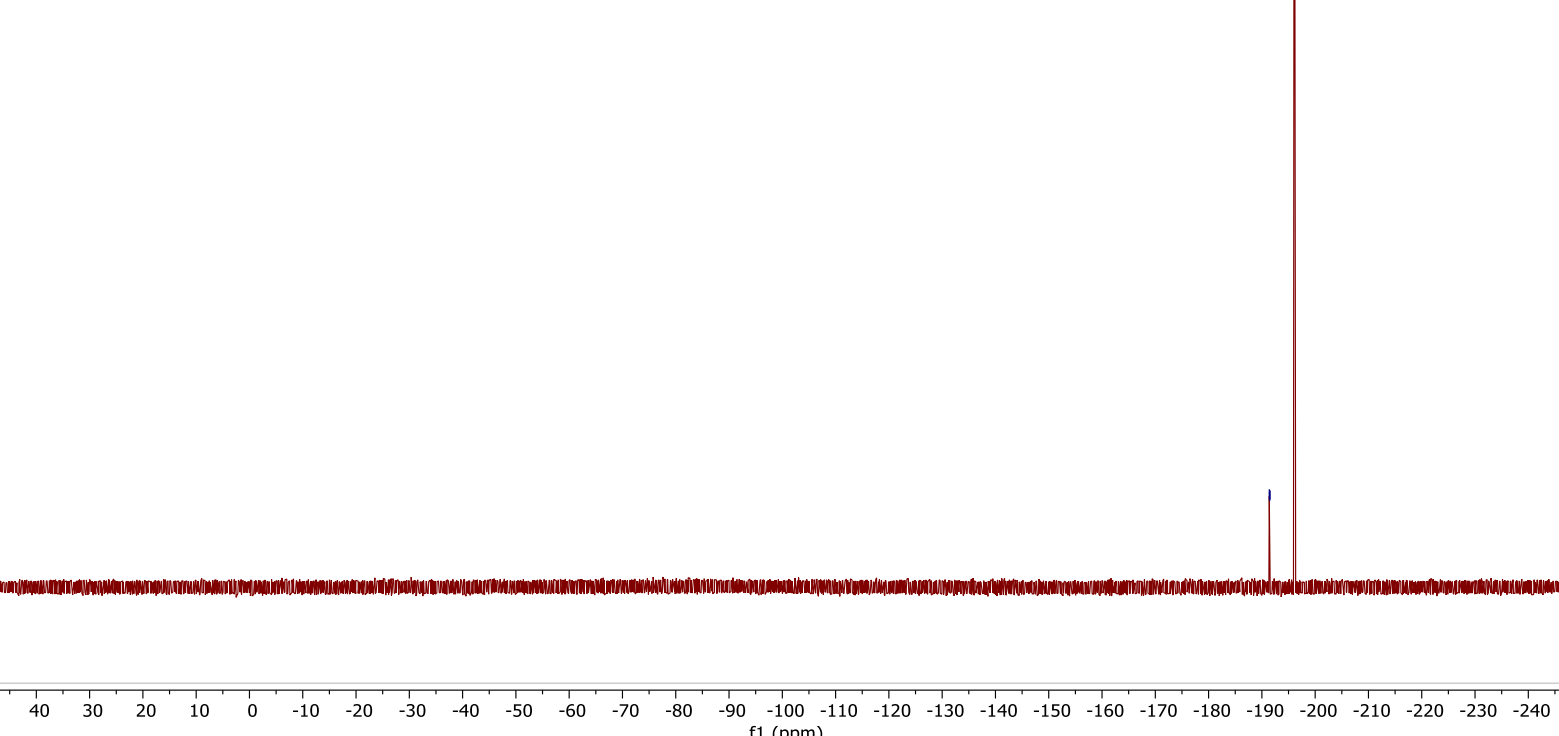

9 - ${ }^{1} \mathrm{H}$ NMR $\left(400 \mathrm{MHz}, \mathrm{CD}_{2} \mathrm{Cl}_{2}\right)$

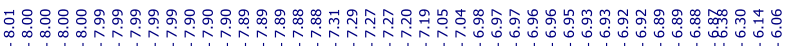
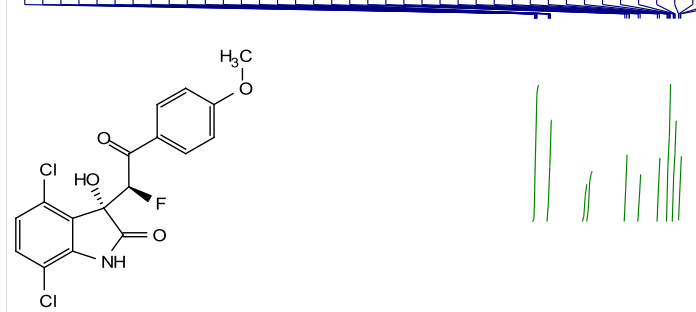

9

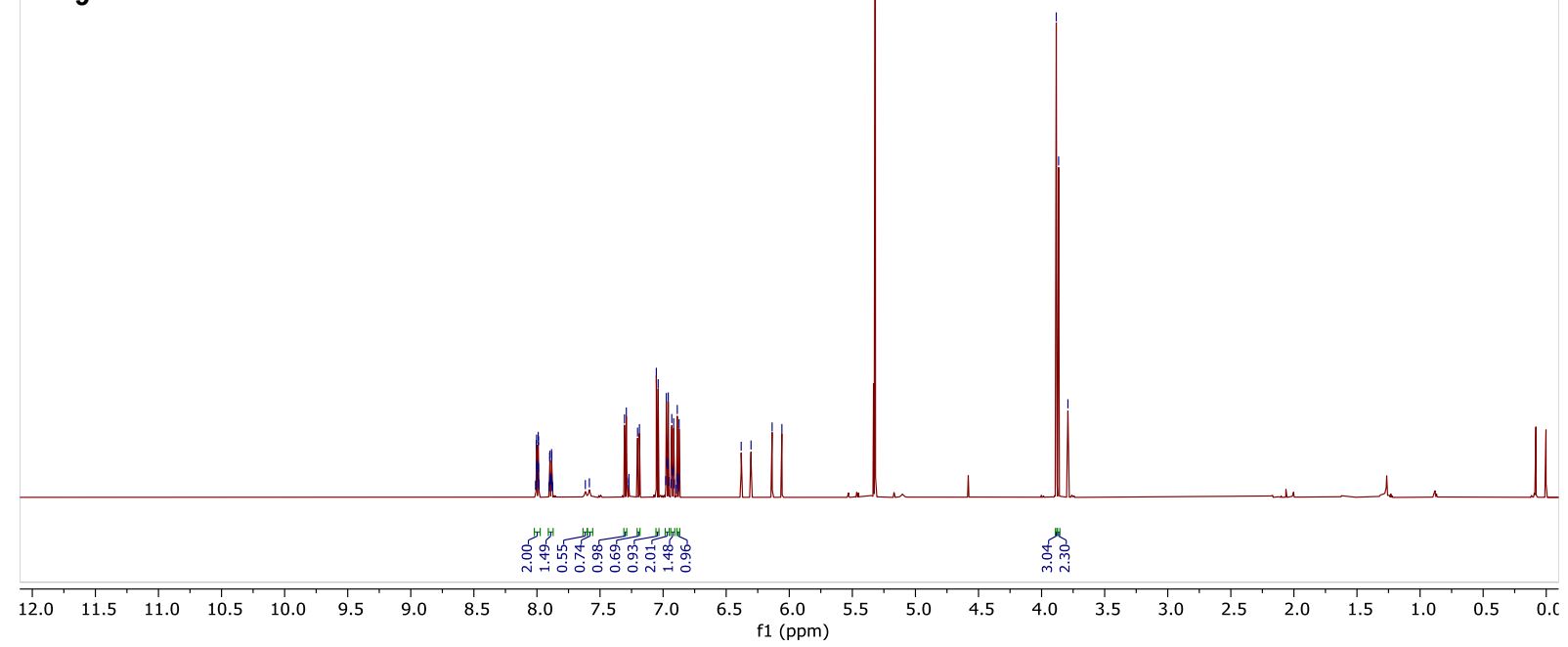


$9-{ }^{13} \mathrm{C}$ NMR $\left(101 \mathrm{MHz}, \mathrm{CD}_{2} \mathrm{Cl}_{2}\right)$

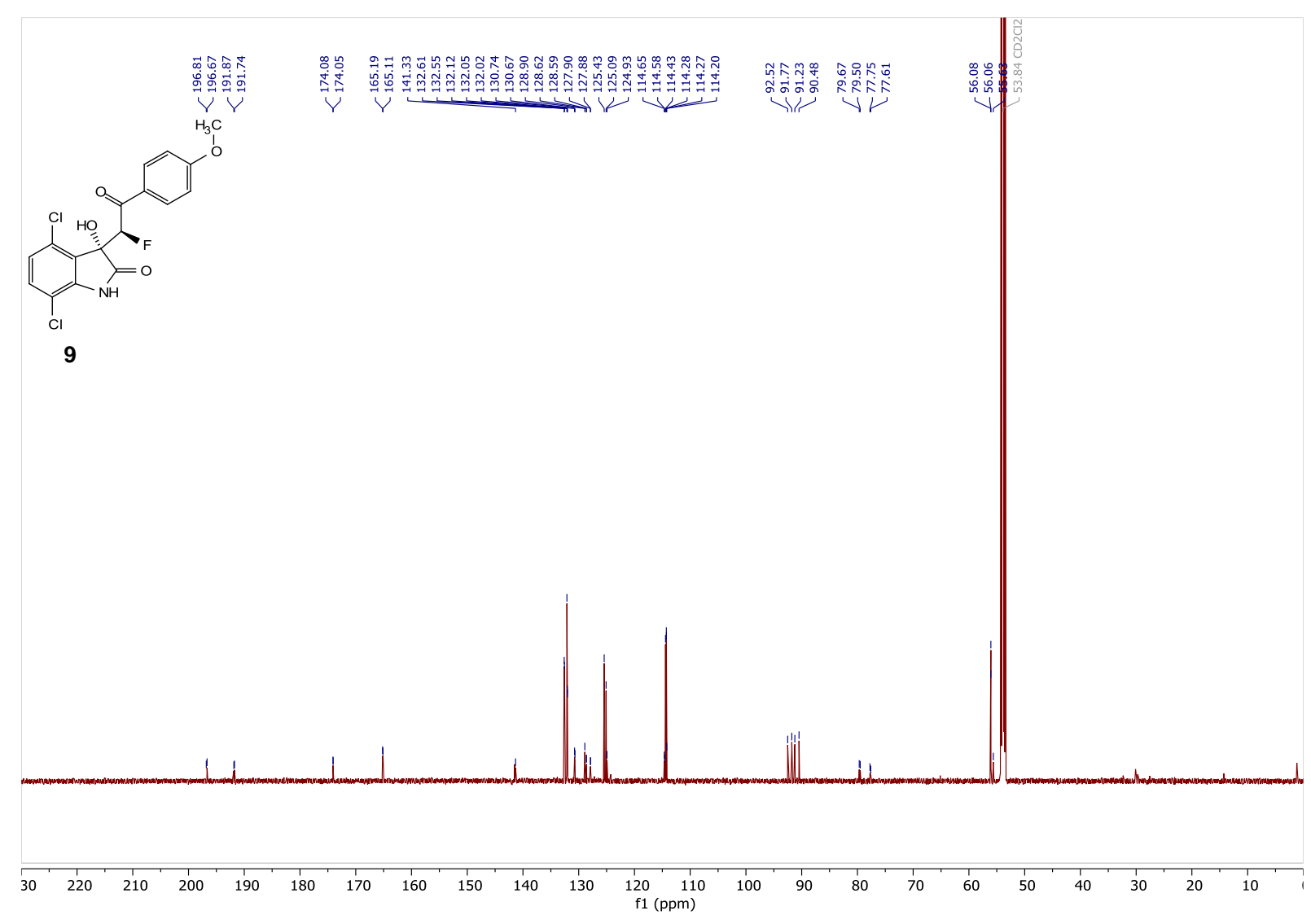

9 - ${ }^{19} \mathrm{~F}$ NMR $\left(376 \mathrm{MHz}, \mathrm{CD}_{2} \mathrm{Cl}_{2}\right)$

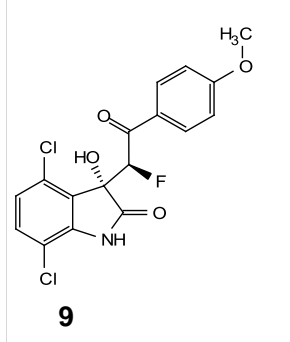

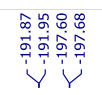

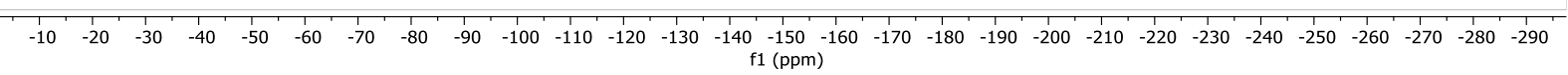


In the following, NMR spectra of $N$-substituted isatins are displayed. For spectral data of previously characterized $\mathrm{N}$-substituted isatins see ref. 4 7.

4-fluoro-1-methylindolin-2,3-dione - ${ }^{1} \mathrm{H}$ NMR $\left(500 \mathrm{MHz}, \mathrm{CDCl}_{3}\right)$

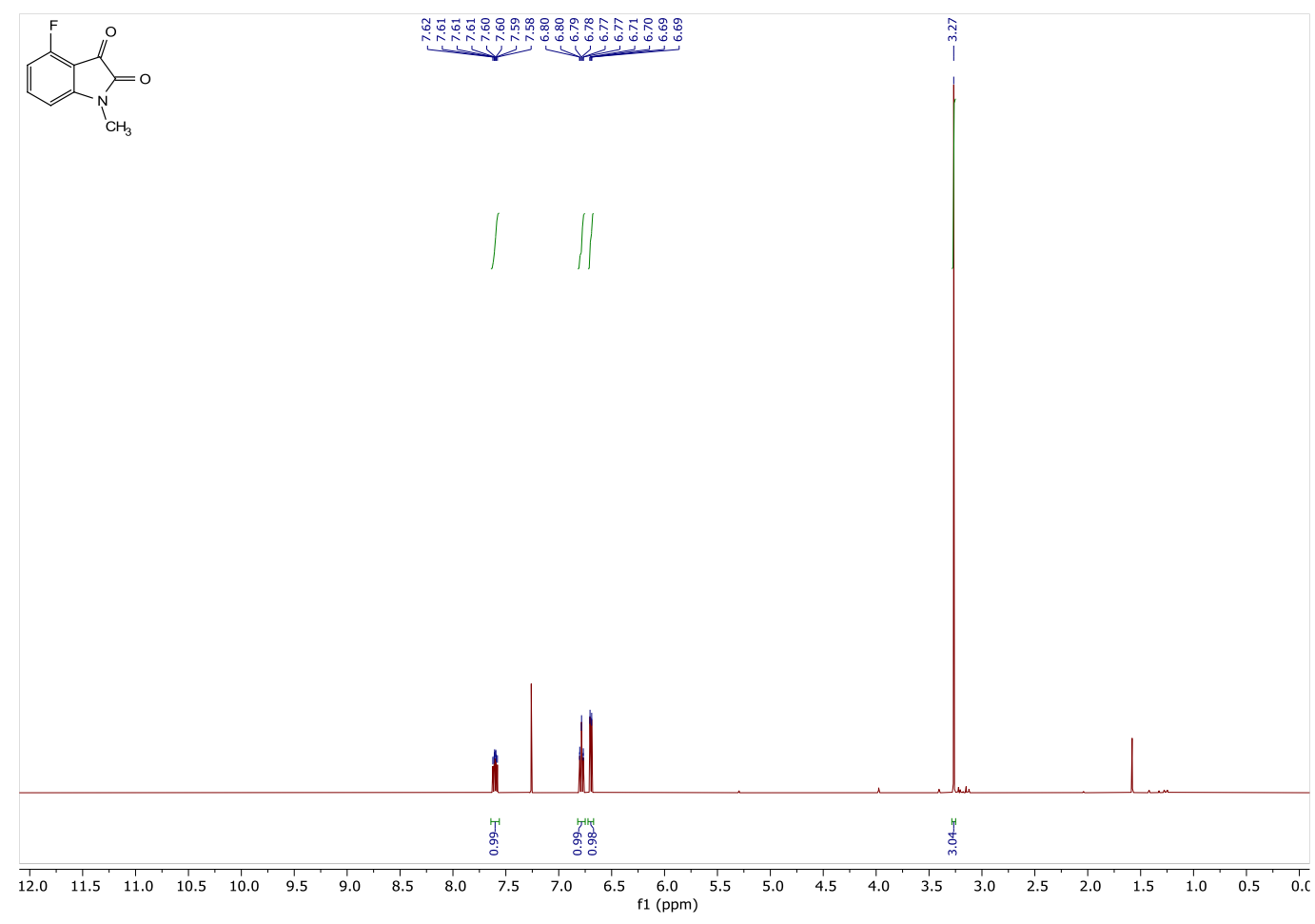

4-fluoro-1-methylindolin-2,3-dione - ${ }^{13} \mathrm{C}$ NMR (126 MHz, $\left.\mathrm{CDCl}_{3}\right)$

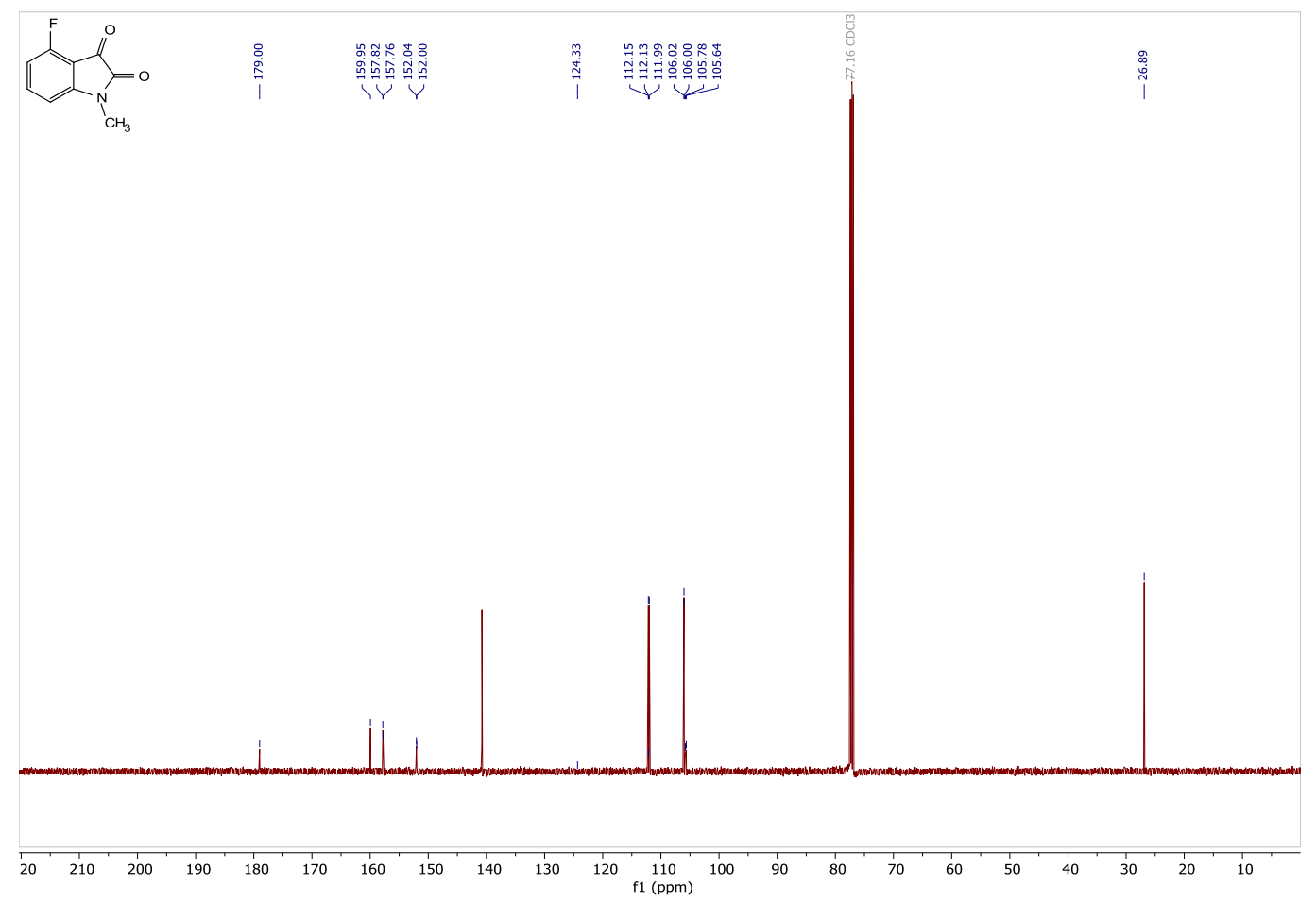


4-fluoro-1-methylindolin-2,3-dione - ${ }^{19} \mathrm{~F}$ NMR (471 MHz, $\left.\mathrm{CDCl}_{3}\right)$

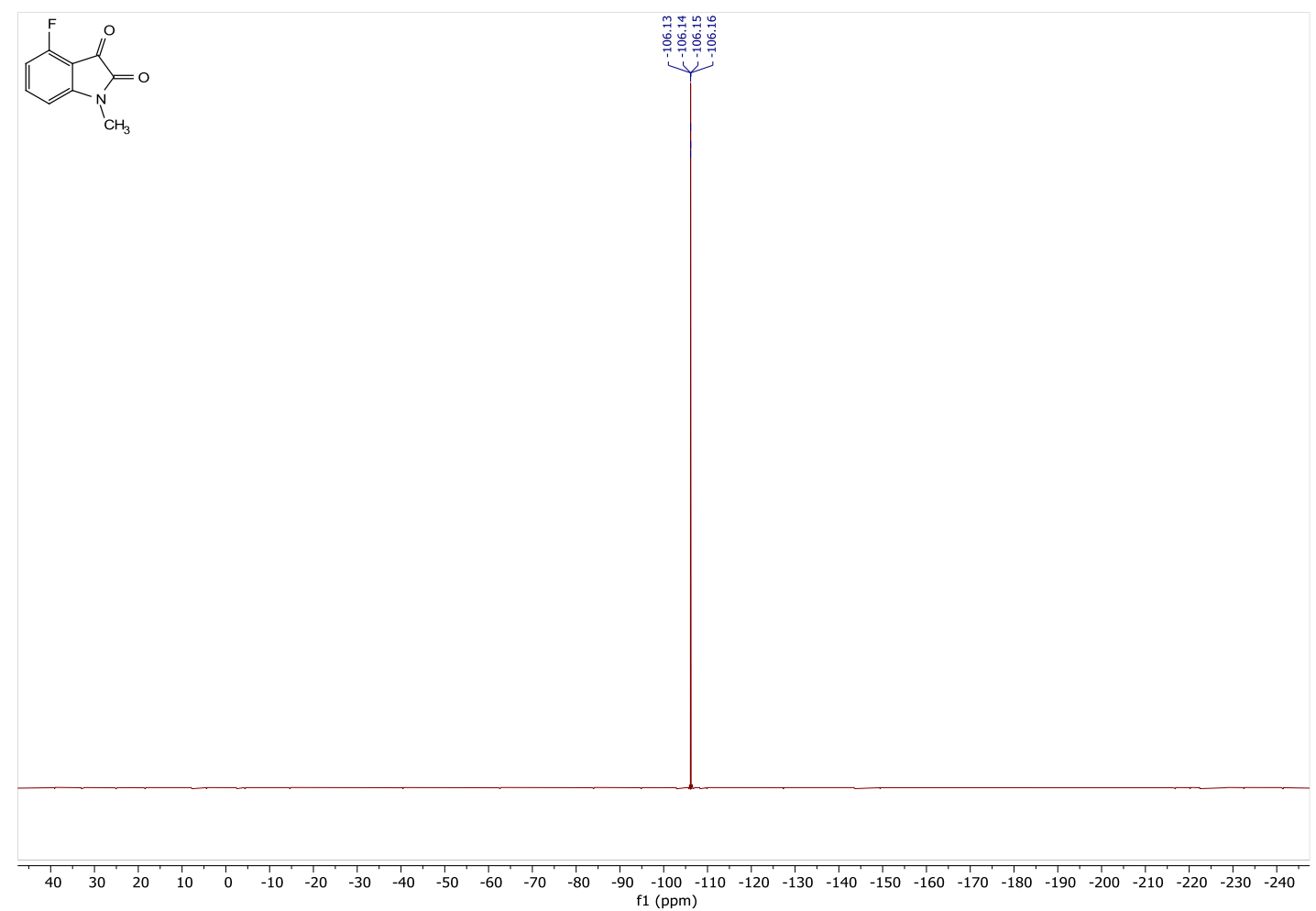

4-chloro-1-methylindolin-2,3-dione - ${ }^{1} \mathrm{H}$ NMR (500 MHz, $\left.\mathrm{CDCl}_{3}\right)$

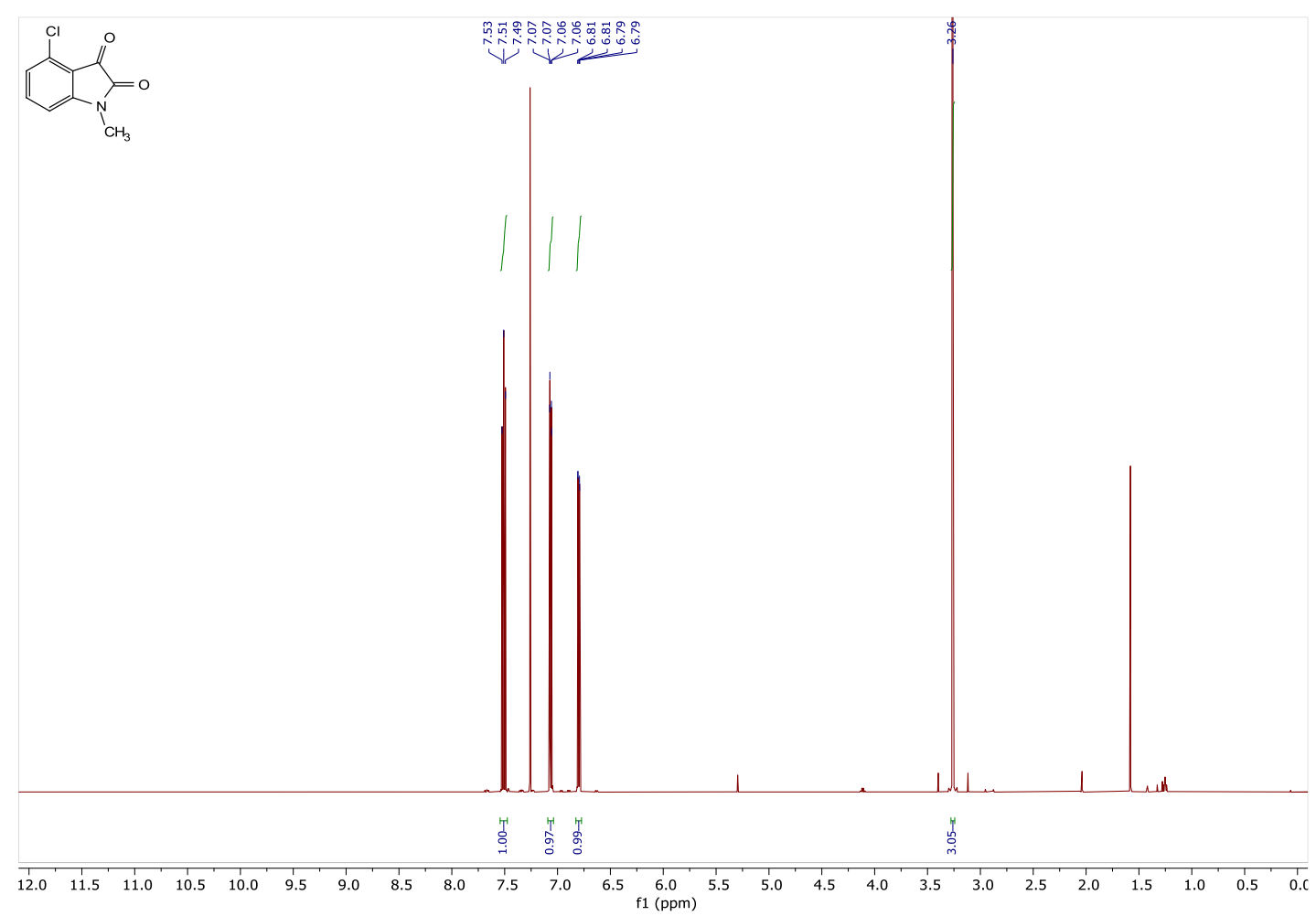


4-chloro-1-methylindolin-2,3-dione - ${ }^{13} \mathrm{C}$ NMR (126 MHz, $\left.\mathrm{CDCl}_{3}\right)$

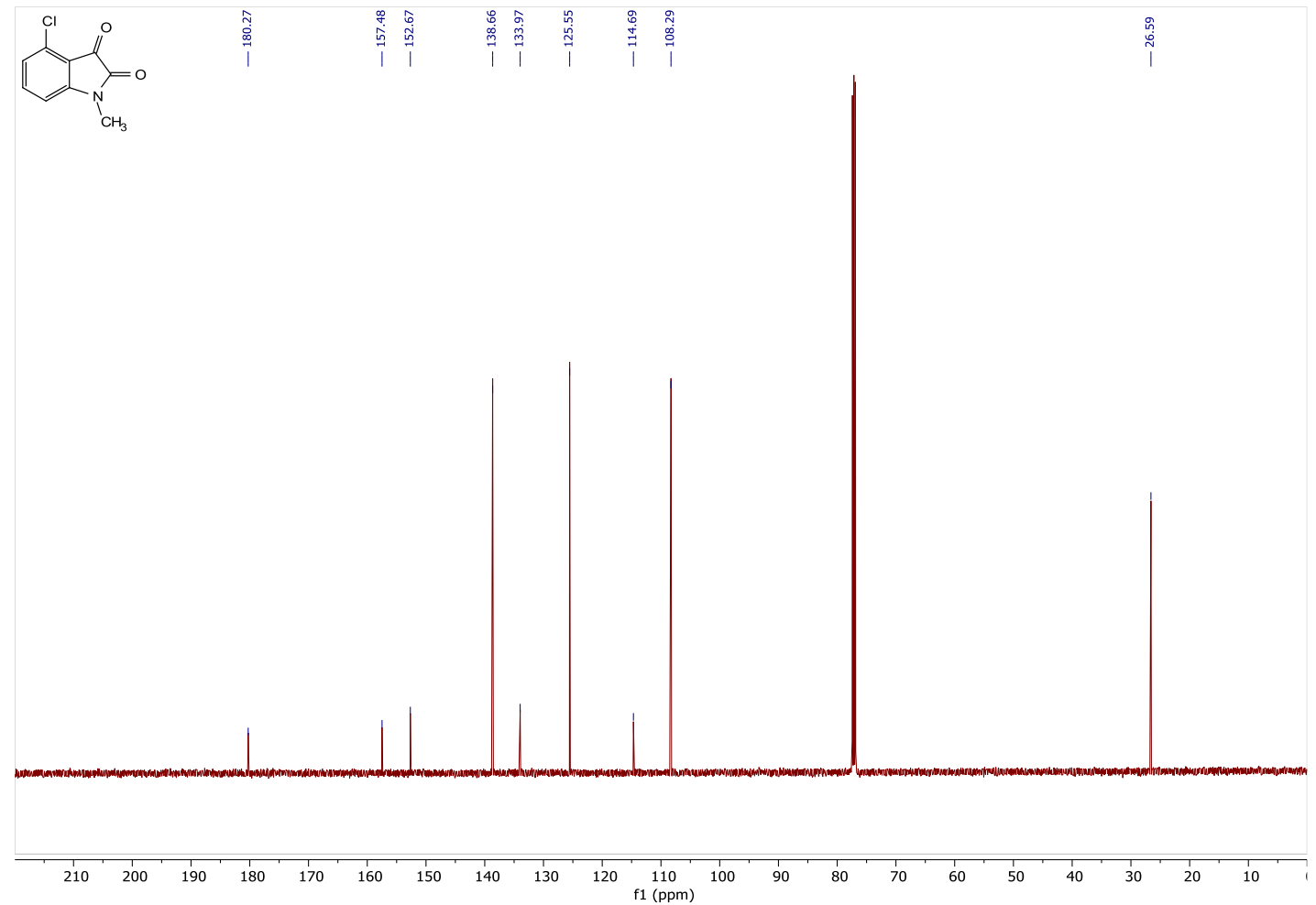

4-bromo-1-methylindolin-2,3-dione - ${ }^{1} \mathrm{H}$ NMR (500 MHz, $\left.\mathrm{CDCl}_{3}\right)$

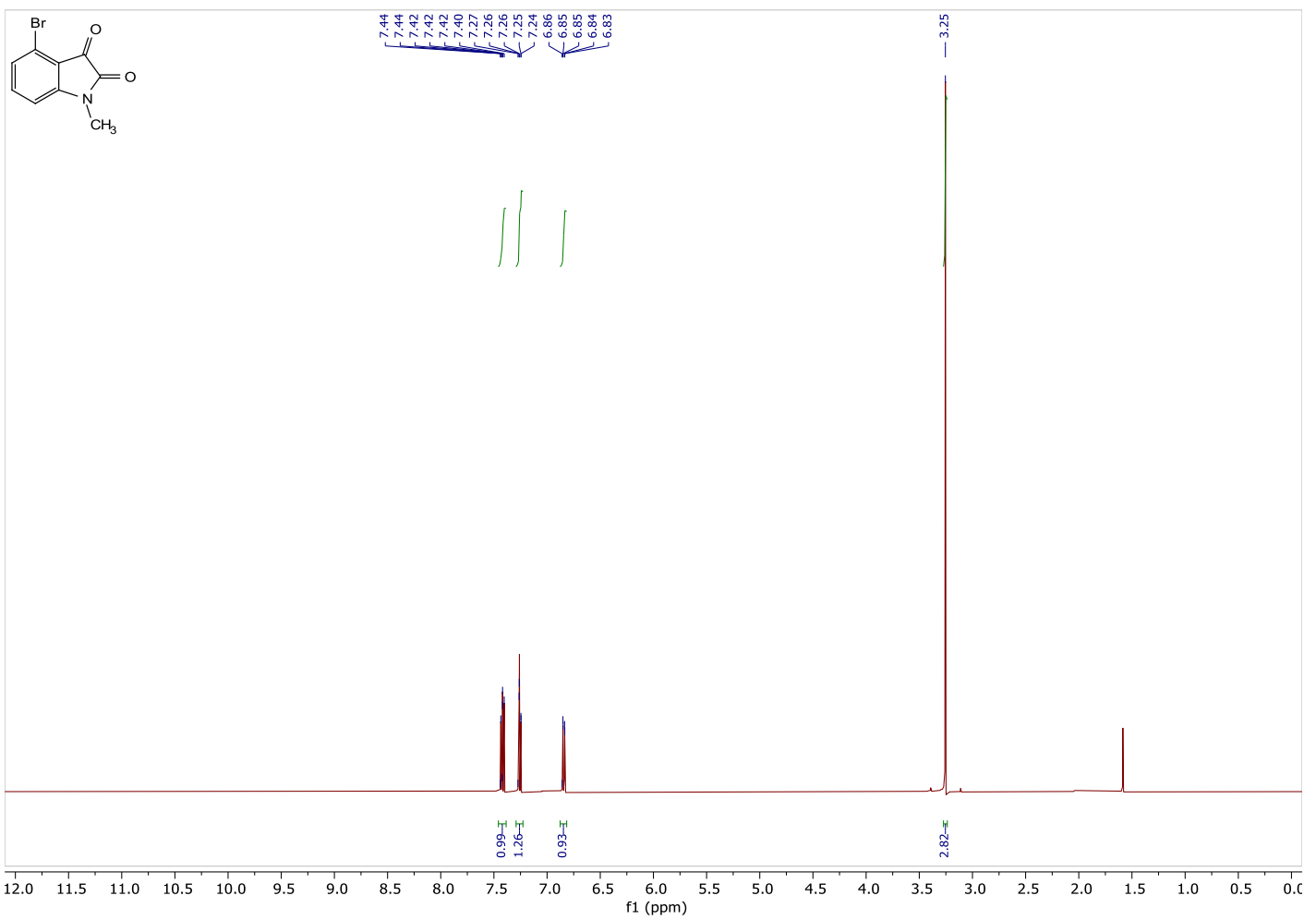


4-bromo-1-methylindolin-2,3-dione - ${ }^{13} \mathrm{C}$ NMR (126 MHz, $\left.\mathrm{CDCl}_{3}\right)$

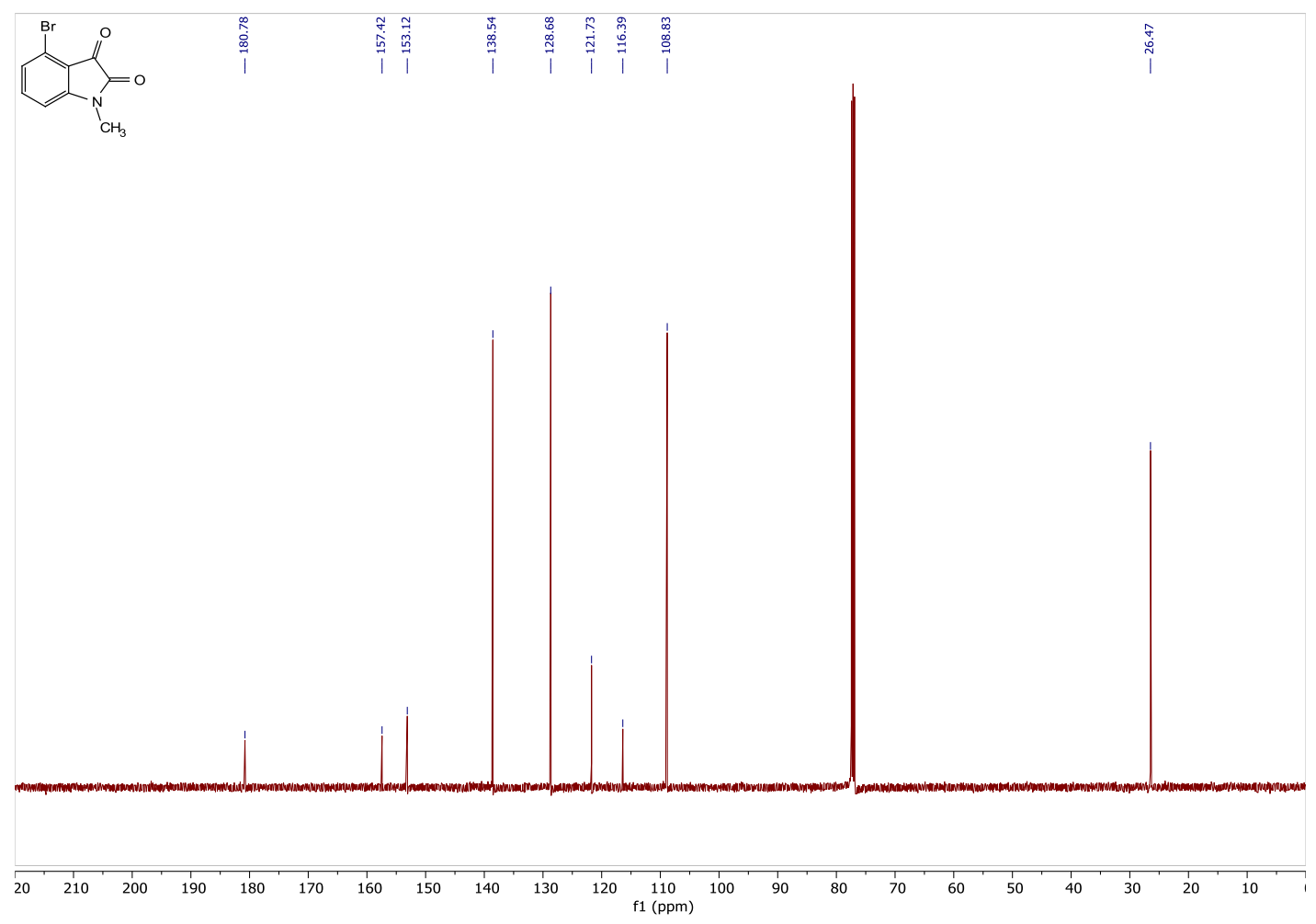

4-phenyl-1-methylindolin-2,3-dione - ${ }^{1} \mathbf{H}$ NMR $\left(500 \mathrm{MHz}, \mathrm{CDCl}_{3}\right)$

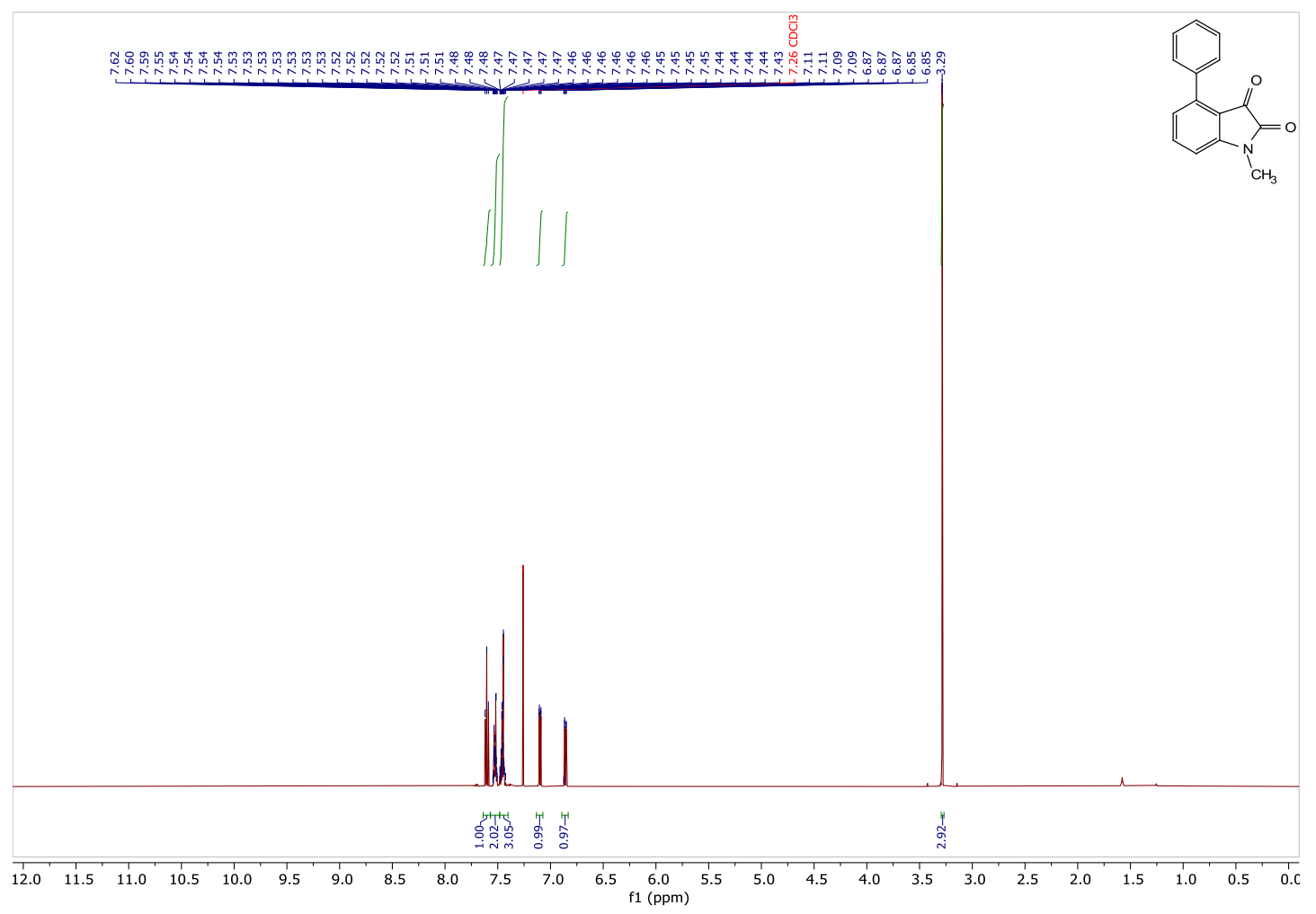




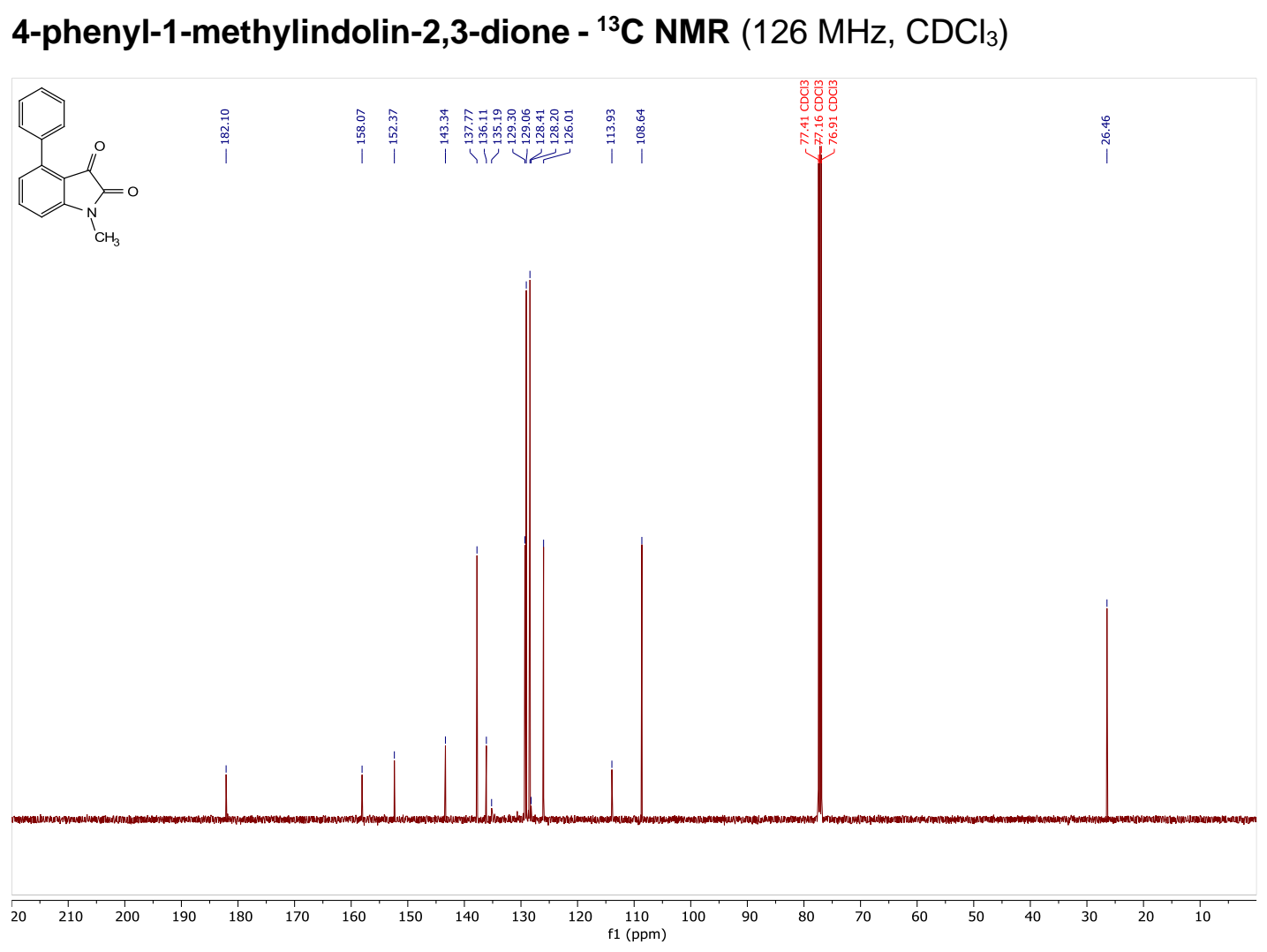

4,7-dichloro-1-methylindoline-2,3-dione - ${ }^{1} \mathrm{H}$ NMR $\left(500 \mathrm{MHz}, \mathrm{CDCl}_{3}\right)$

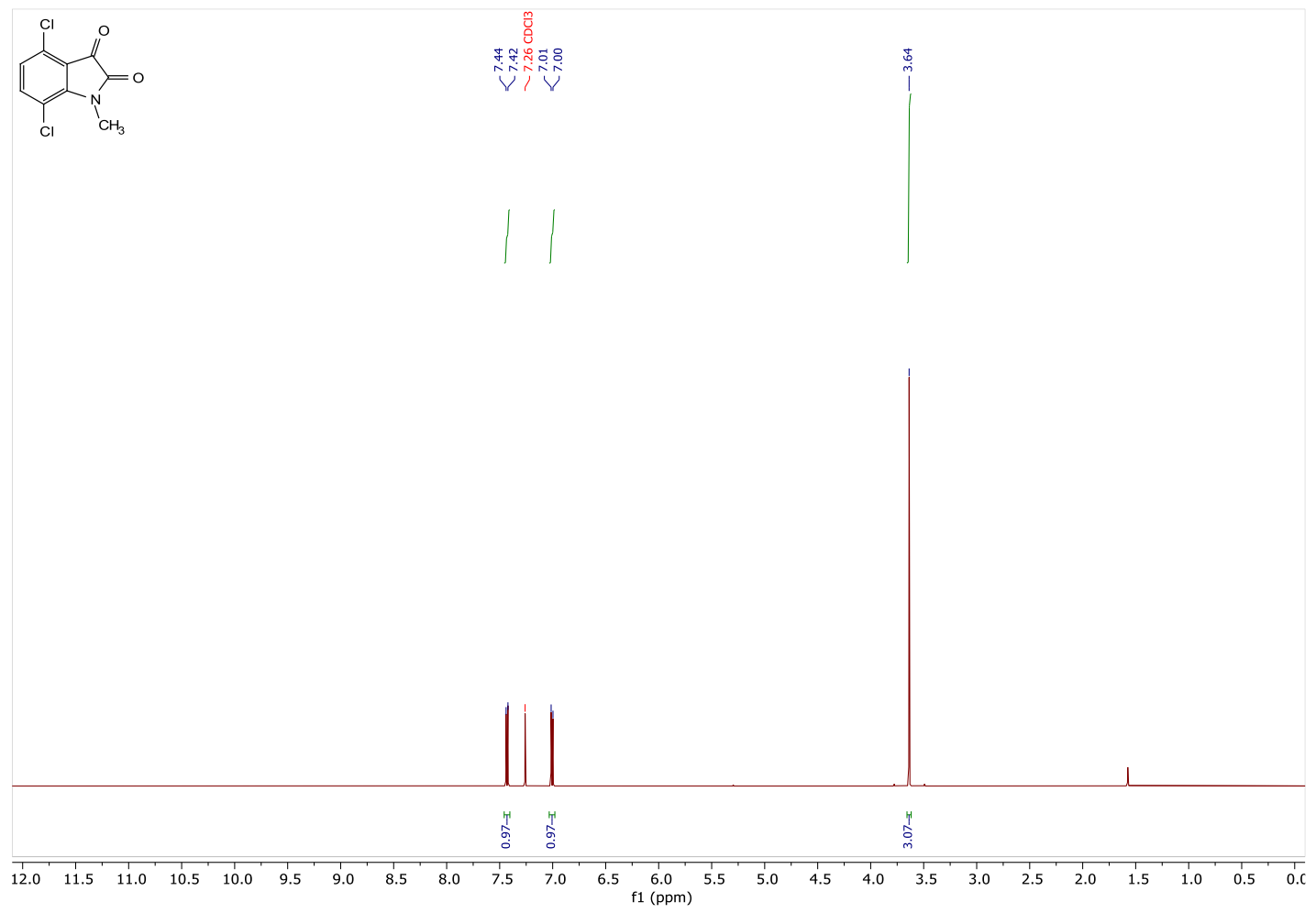


4,7-dichloro-1-methylindoline-2,3-dione - $\left.{ }^{13} \mathrm{C} \mathrm{NMR} \mathrm{(126} \mathrm{MHz,} \mathrm{CDCl}_{3}\right)$

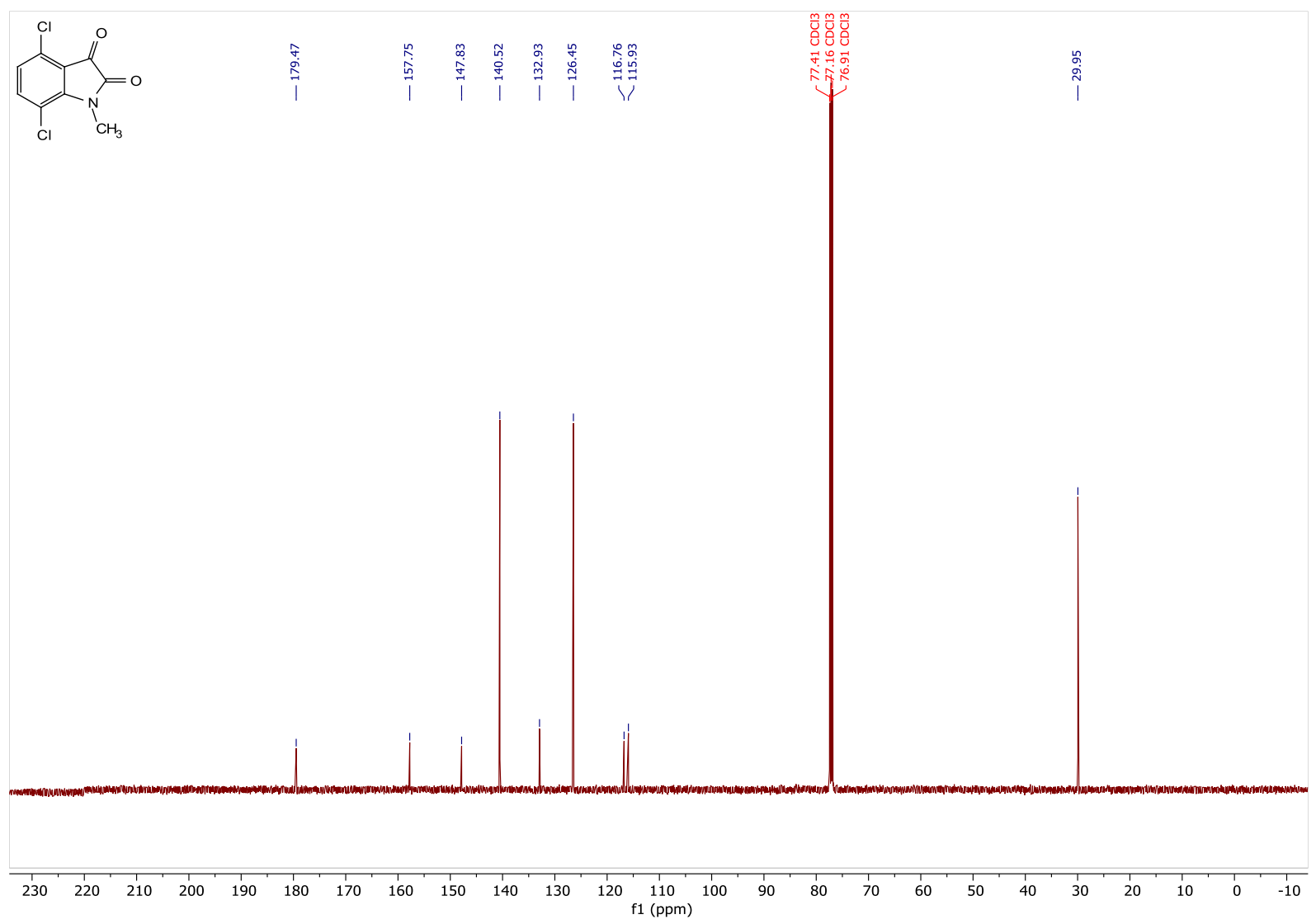

1-methyl-5-(trifluoromethoxy)indoline-2,3-dione - ${ }^{1} \mathbf{H}$ NMR $\left(500 \mathrm{MHz}, \mathrm{CDCl}_{3}\right)$

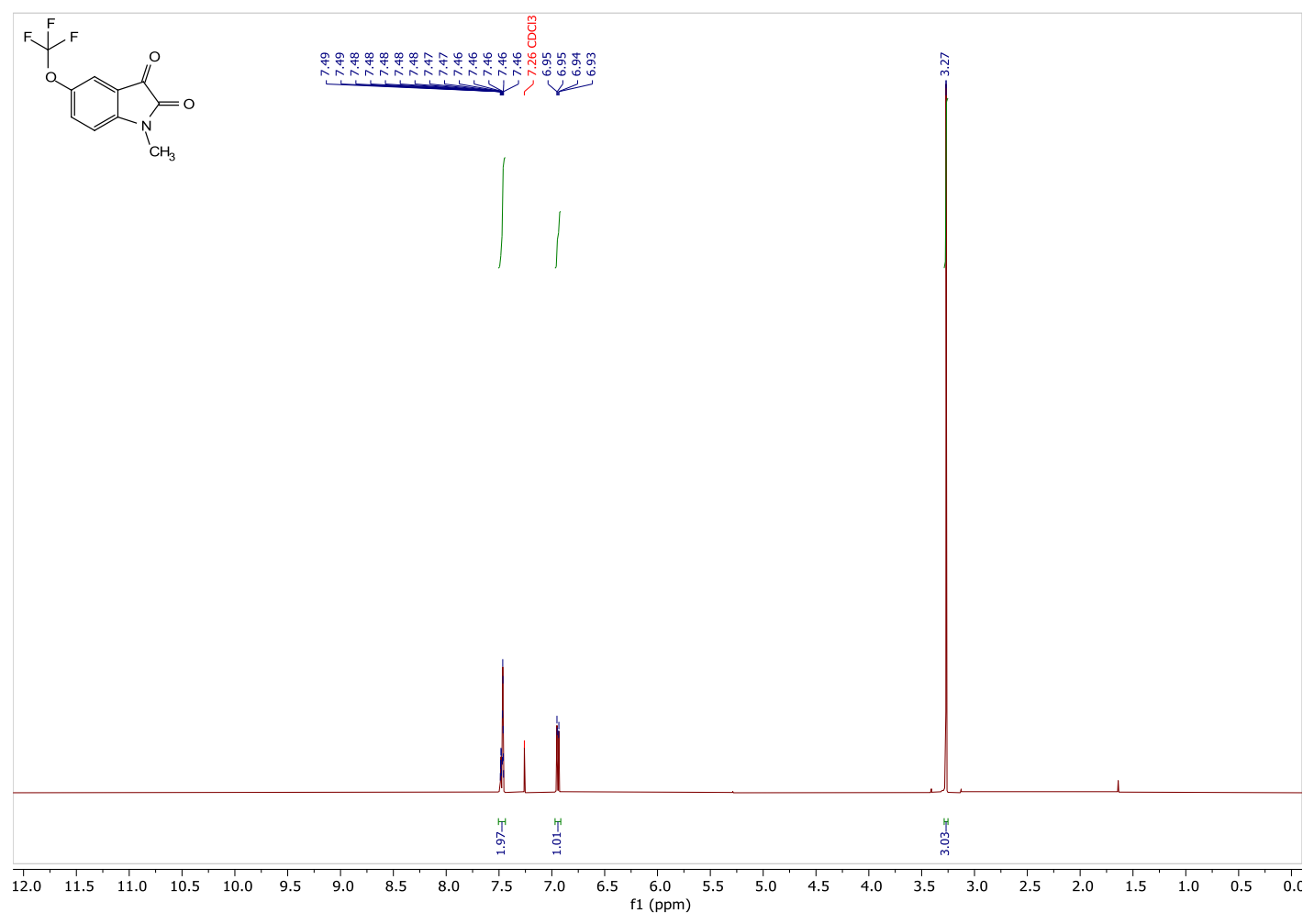


1-methyl-5-(trifluoromethoxy)indoline-2,3-dione - ${ }^{13} \mathrm{C}$ NMR $\left(126 \mathrm{MHz}, \mathrm{CDCl}_{3}\right)$
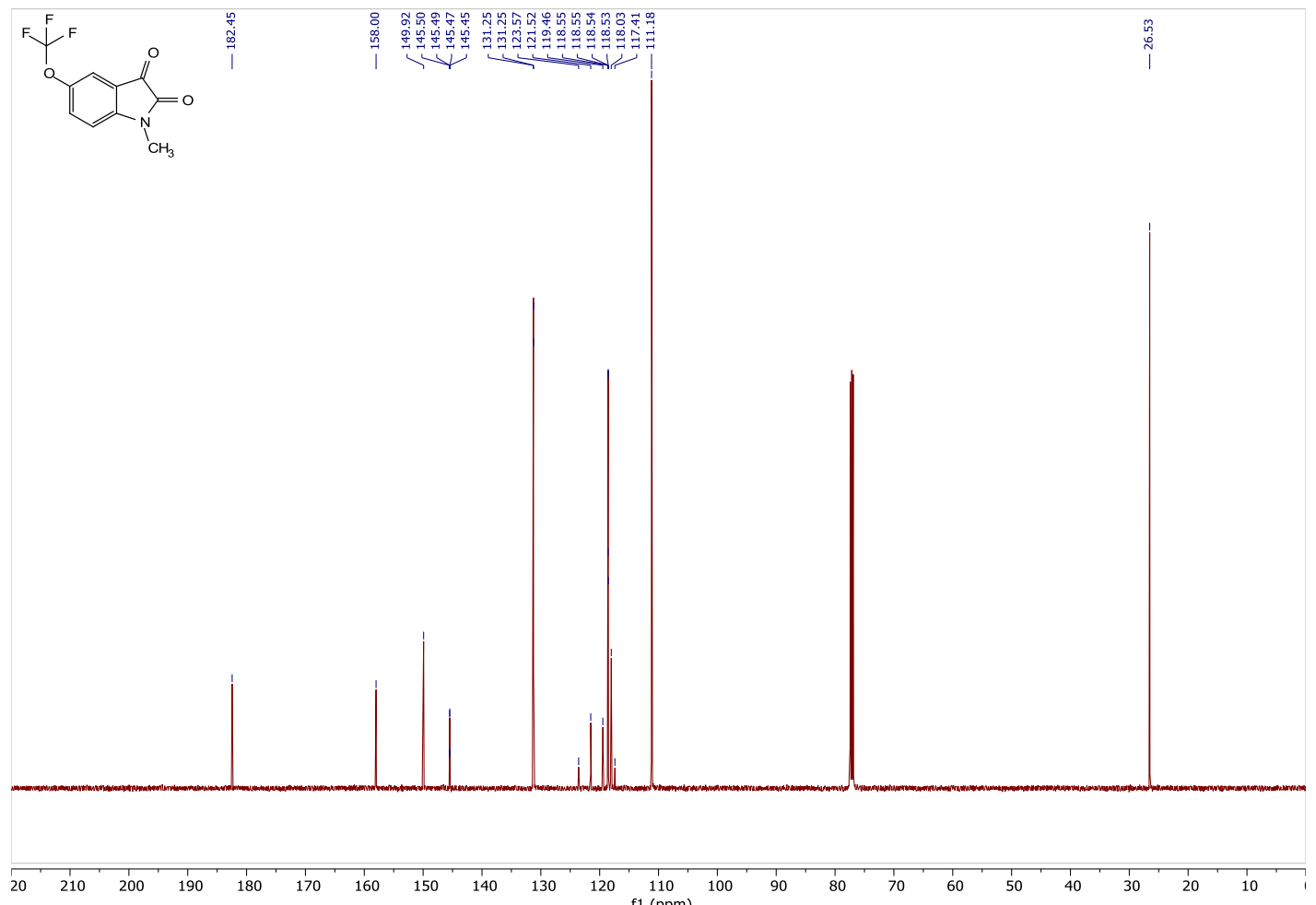

1-methyl-5-(trifluoromethoxy)indoline-2,3-dione - ${ }^{19} \mathrm{~F}$ NMR $\left(471 \mathrm{MHz}, \mathrm{CDCl}_{3}\right)$

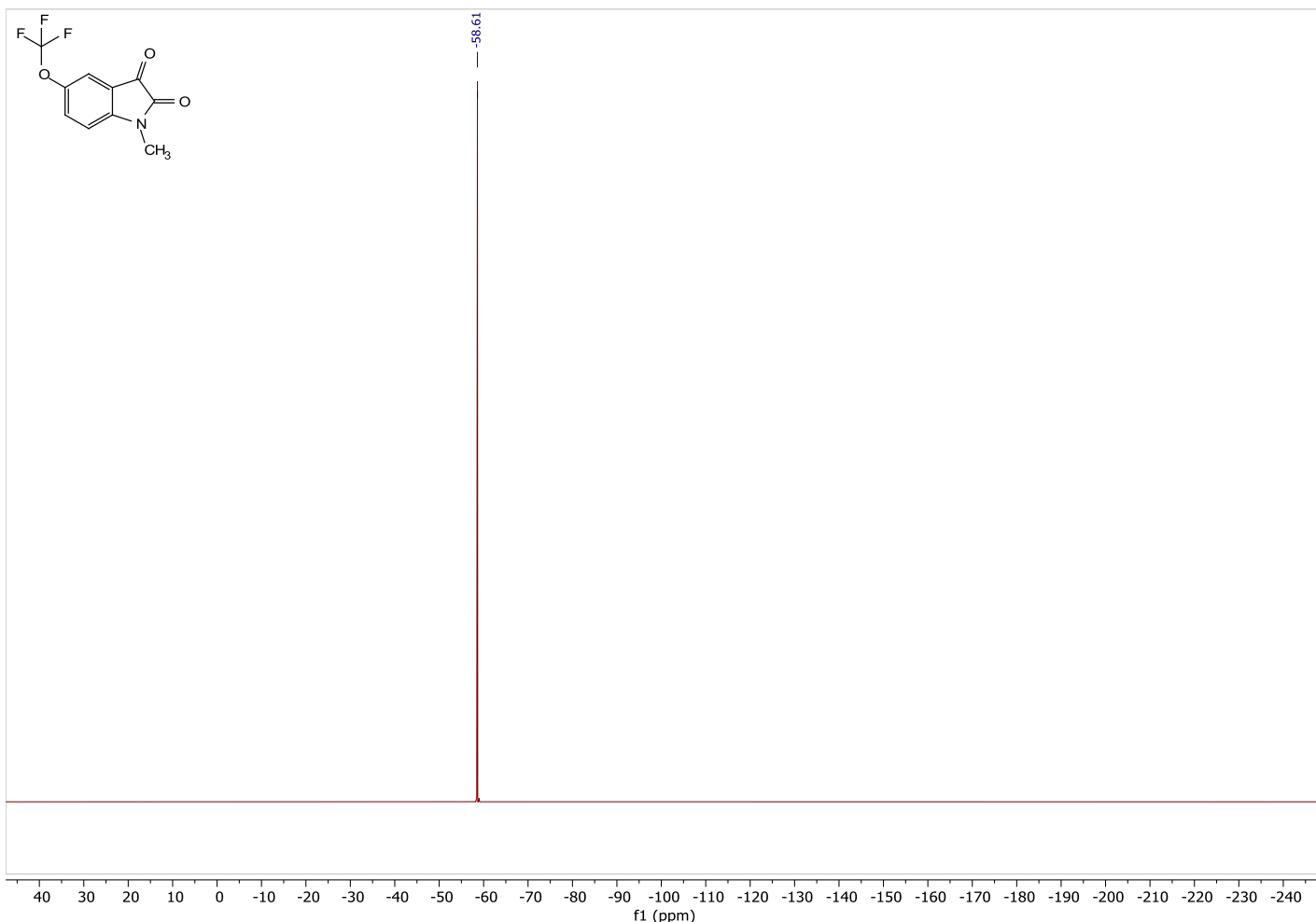


1-methyl-7-(trifluoromethyl)indoline-2,3-dione - ${ }^{1} \mathbf{H}$ NMR $\left(400 \mathrm{MHz}, \mathrm{CDCl}_{3}\right)$

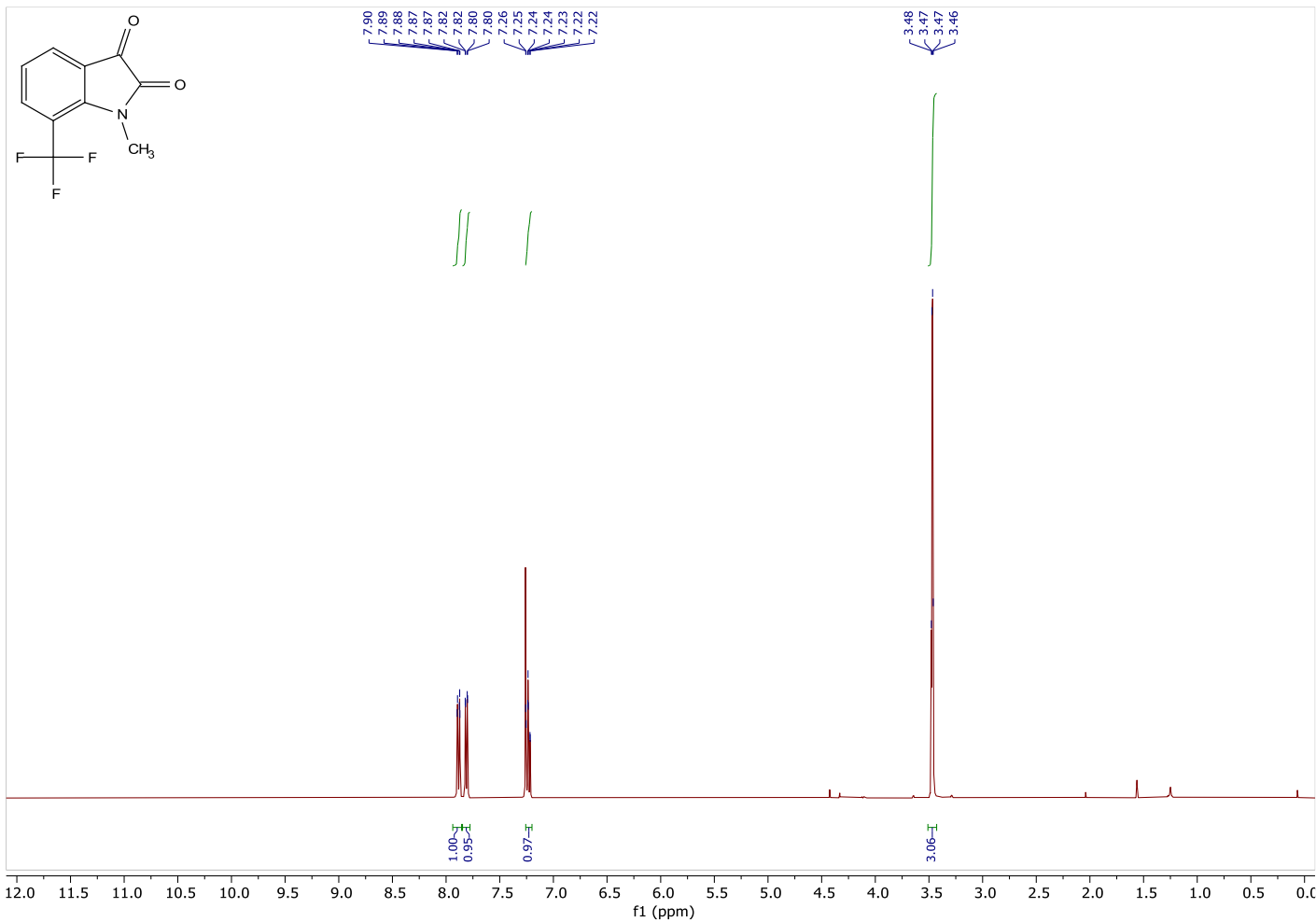

1-methyl-7-(trifluoromethyl)indoline-2,3-dione - ${ }^{13} \mathrm{C}$ NMR $\left(101 \mathrm{MHz}, \mathrm{CDCl}_{3}\right)$
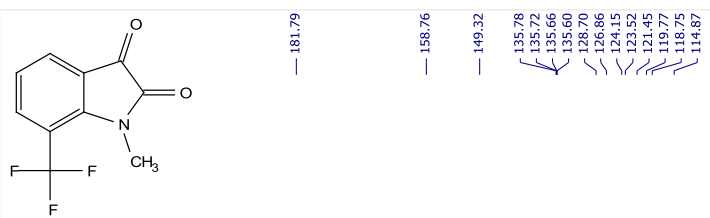

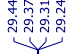

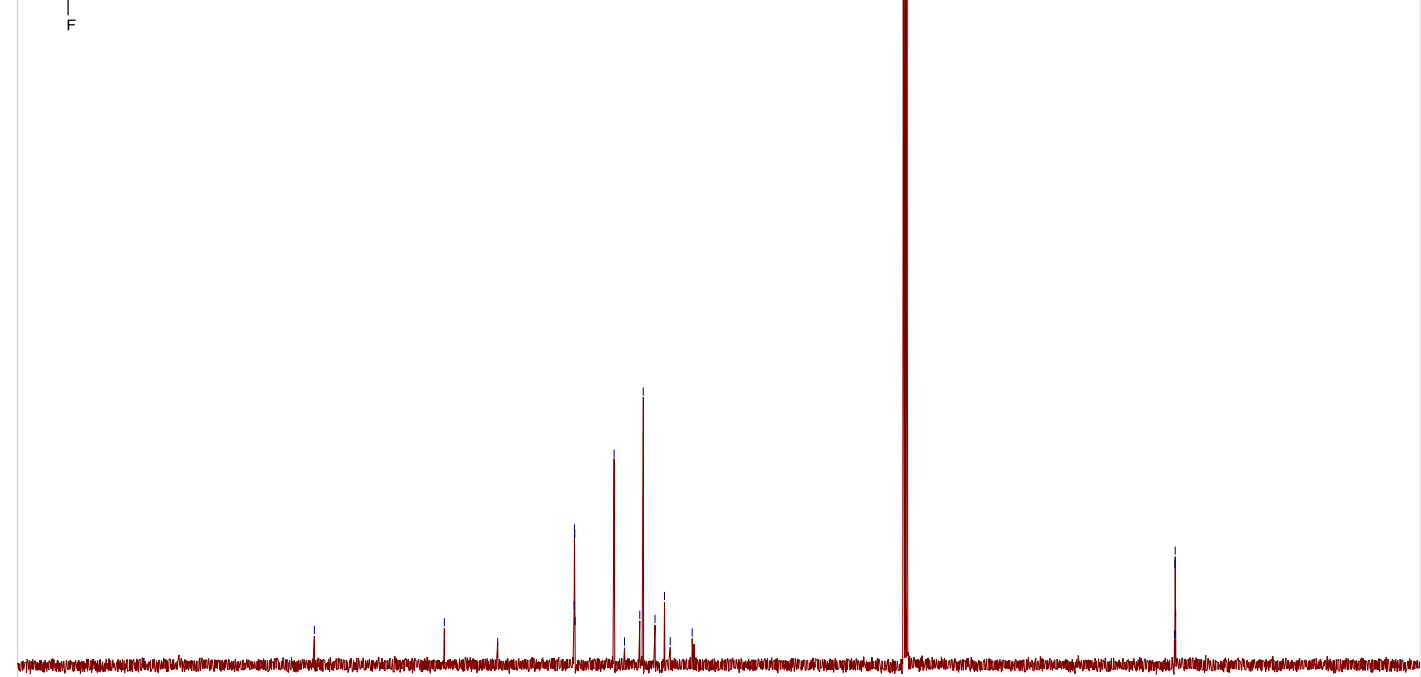

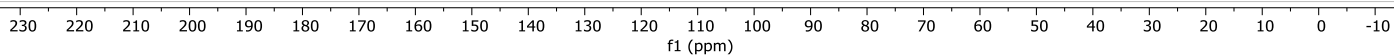


1-methyl-7-(trifluoromethyl)indoline-2,3-dione - ${ }^{19} \mathrm{~F}$ NMR $\left(376 \mathrm{MHz}, \mathrm{CDCl}_{3}\right)$

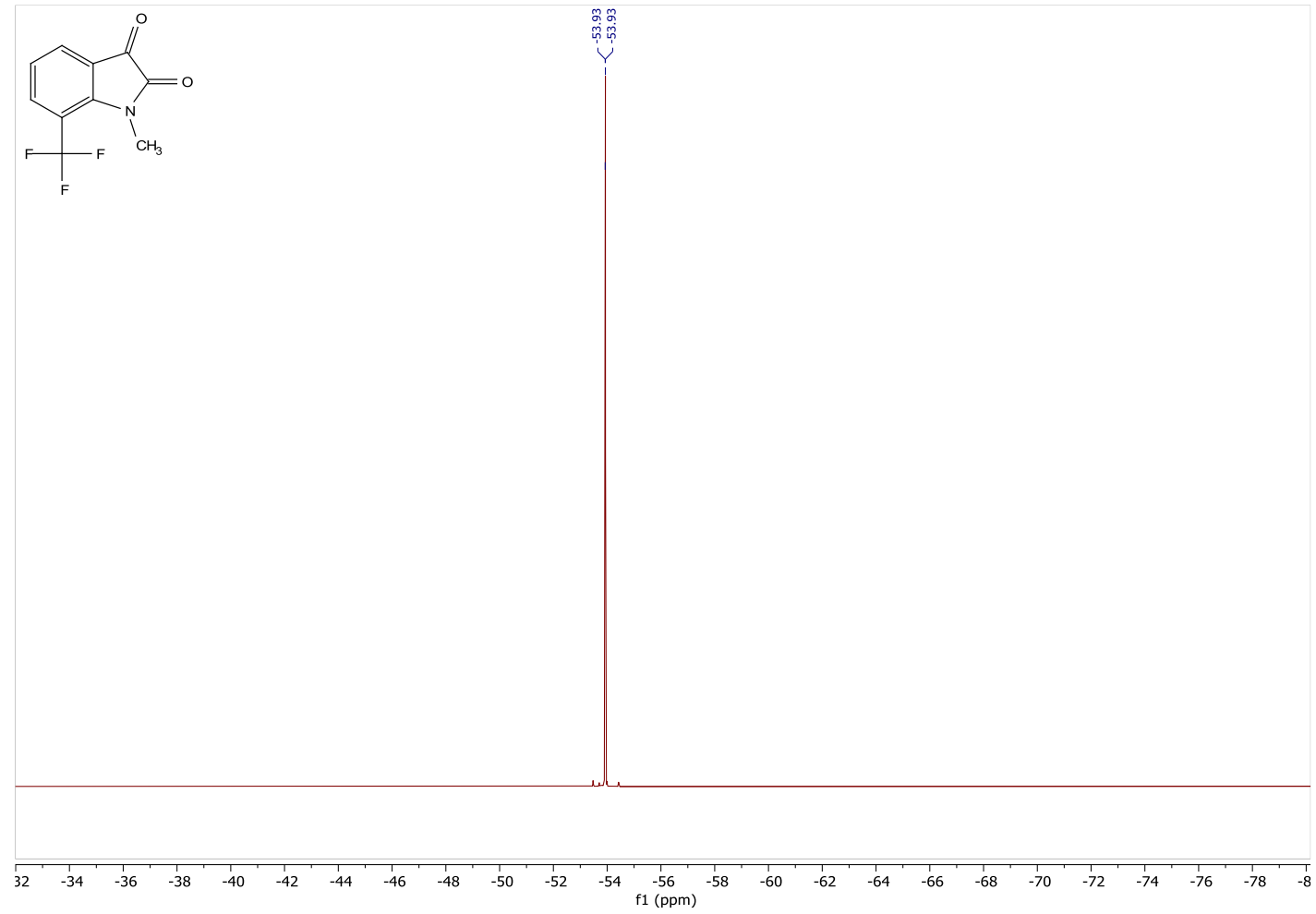

Methyl 1-methyl-2,3-dioxoindoline-7-carboxylate - ${ }^{1} \mathbf{H}$ NMR $\left(400 \mathrm{MHz}, \mathrm{CDCl}_{3}\right)$

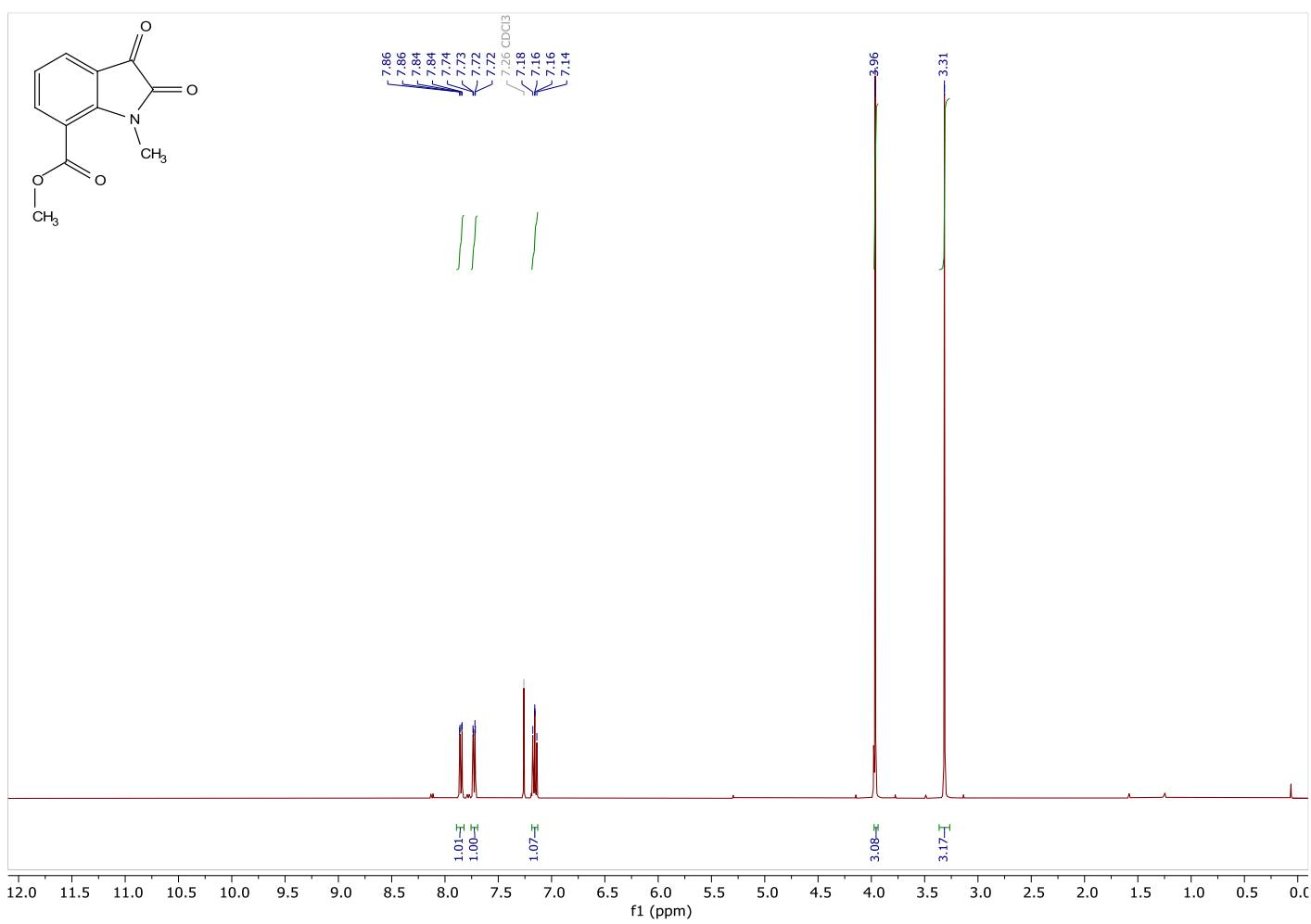


Methyl 1-methyl-2,3-dioxoindoline-7-carboxylate $-{ }^{13} \mathrm{C}$ NMR $\left(101 \mathrm{MHz}, \mathrm{CDCl}_{3}\right)$

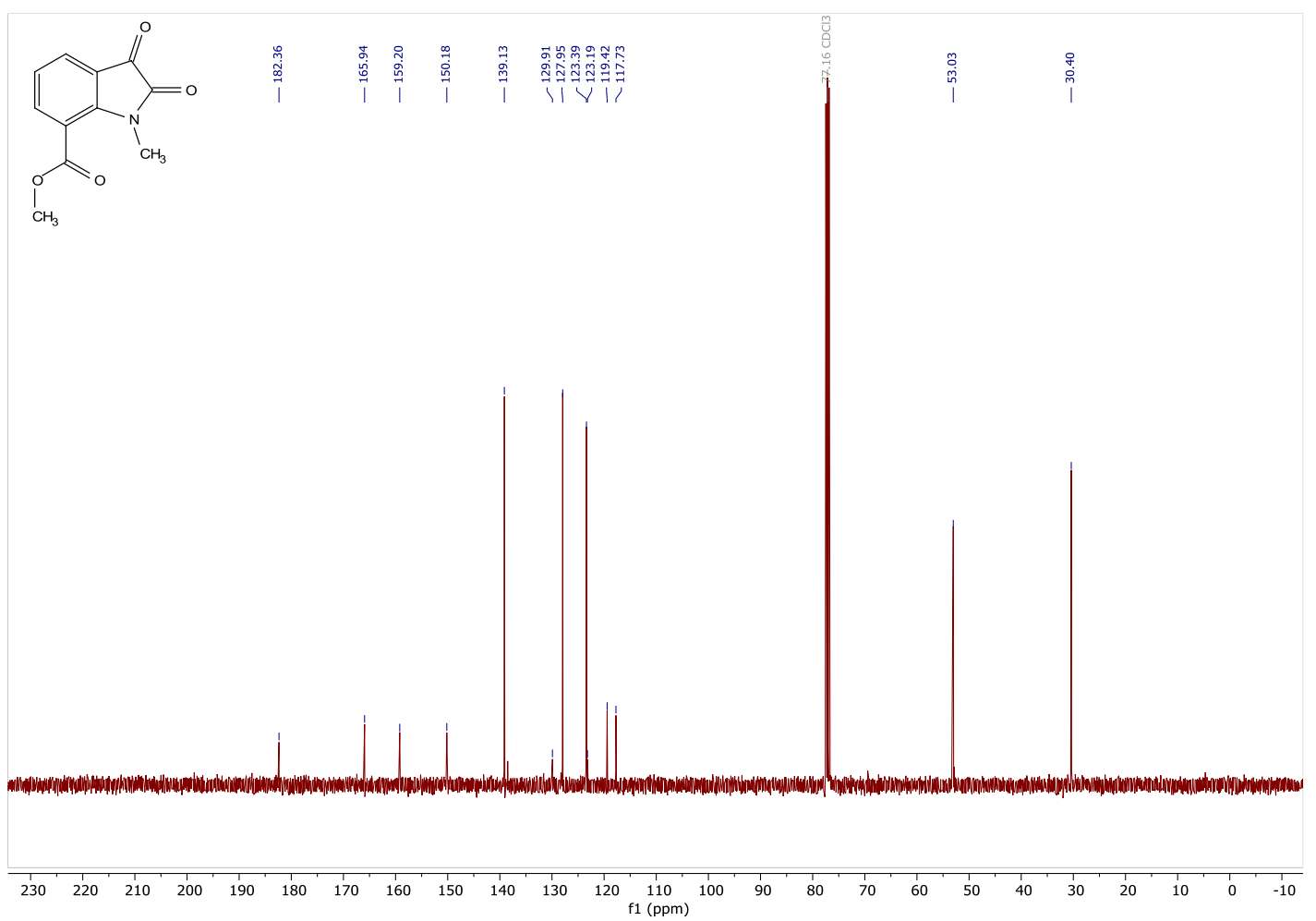

5,7-dibromo-1-methylindoline-2,3-dione - ${ }^{1} \mathbf{H}$ NMR (400 MHz, $\left.\mathrm{CDCl}_{3}\right)$
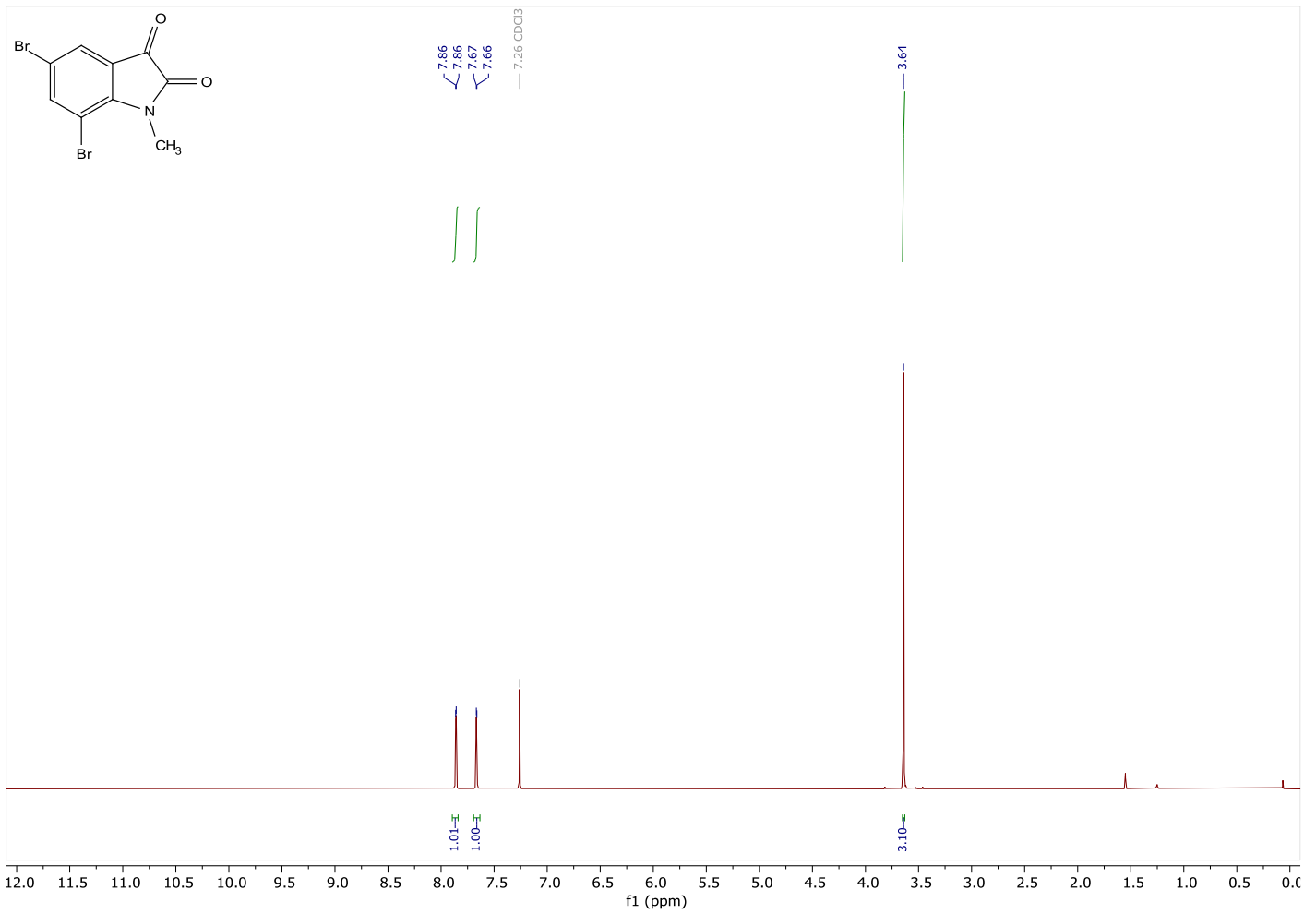
5,7-dibromo-1-methylindoline-2,3-dione - ${ }^{13} \mathrm{C}$ NMR $\left(101 \mathrm{MHz}, \mathrm{CDCl}_{3}\right)$

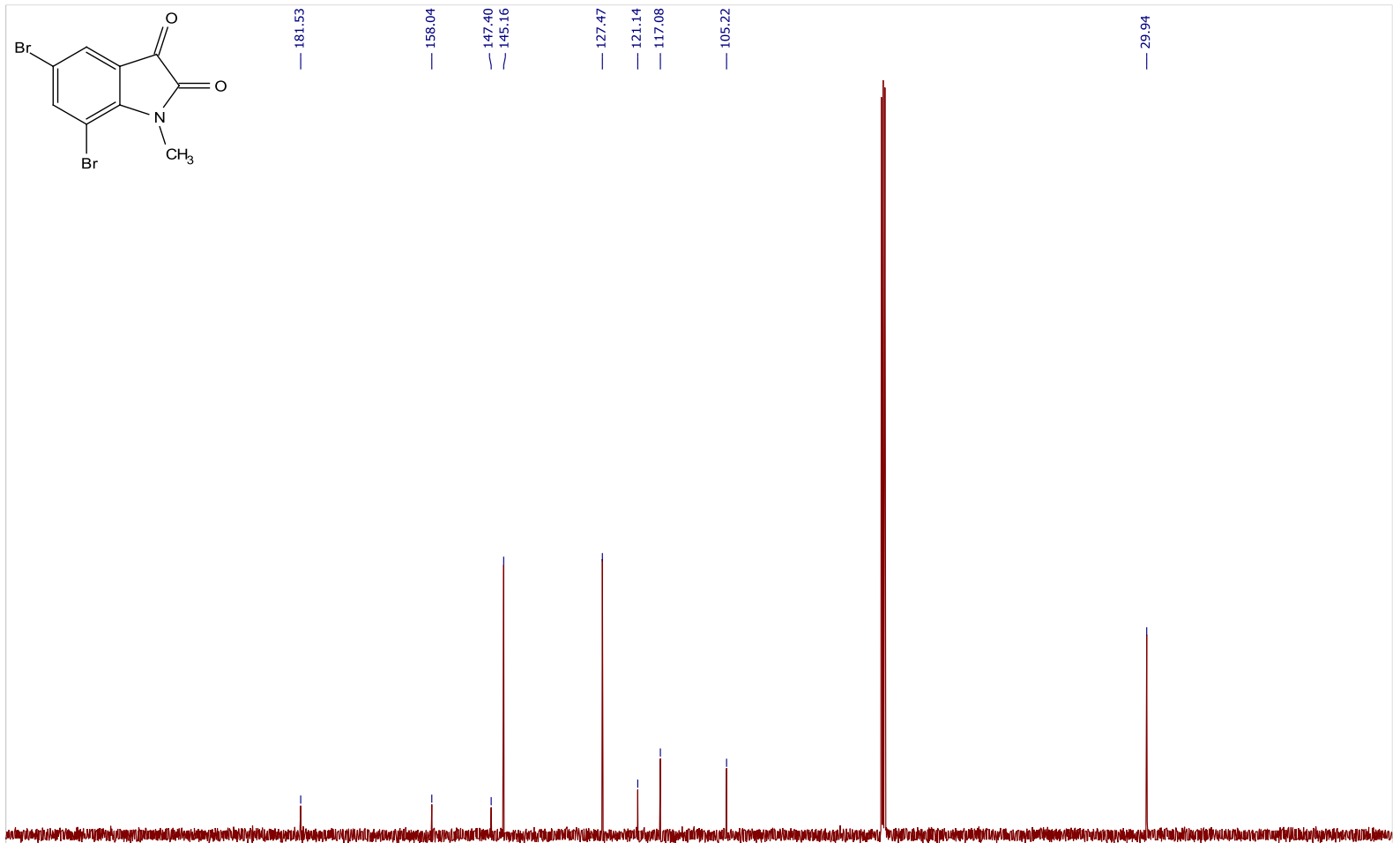

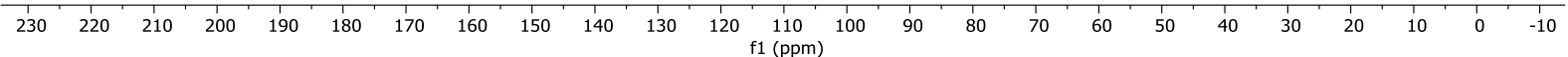

1,5-dimethylindoline-2,3-dione - ${ }^{1} \mathrm{H}$ NMR $\left(400 \mathrm{MHz}, \mathrm{CDCl}_{3}\right)$

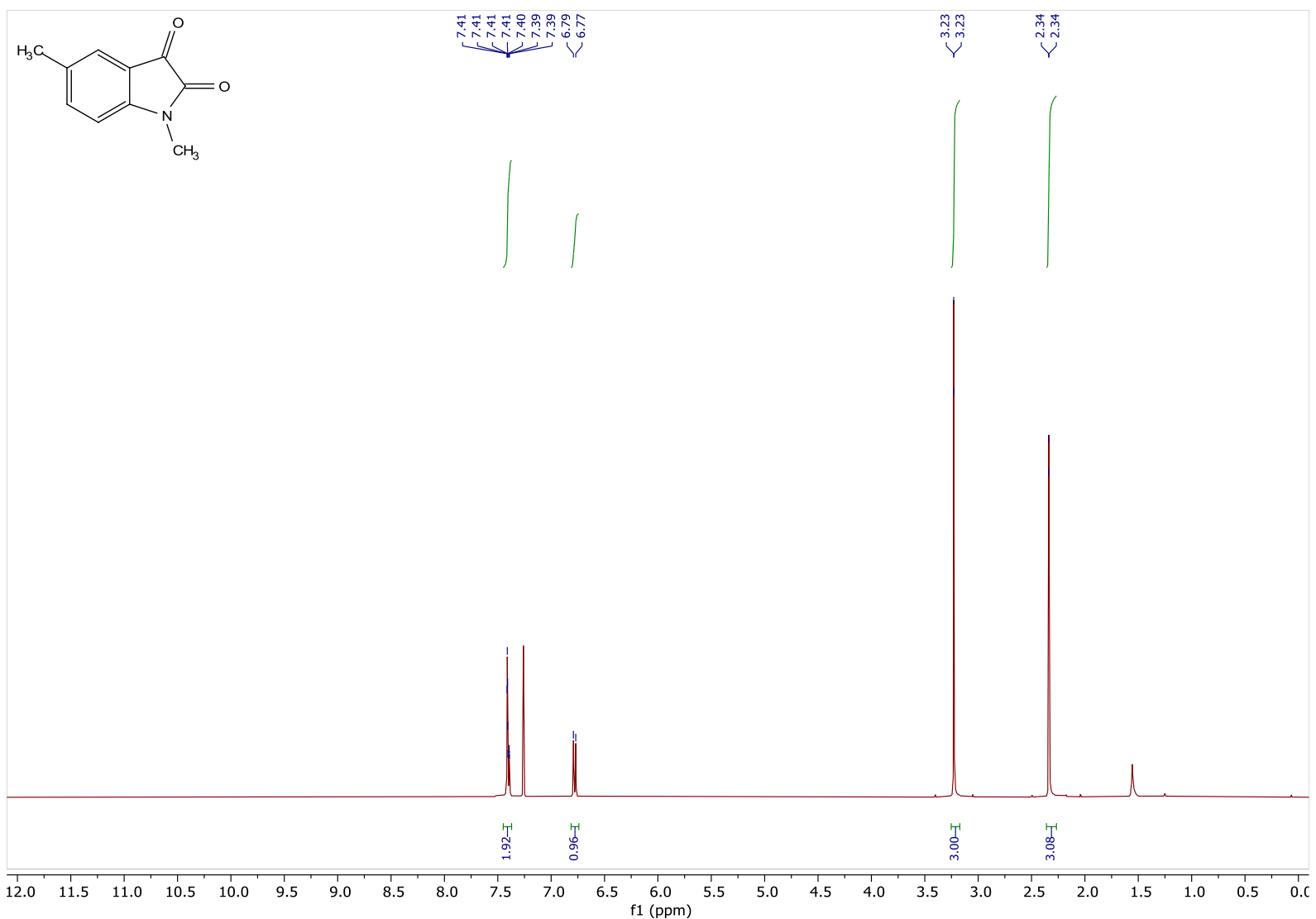


5-methoxy-1-methylindoline-2,3-dione - ${ }^{1} \mathrm{H}$ NMR (400 MHz, $\left.\mathrm{CDCl}_{3}\right)$

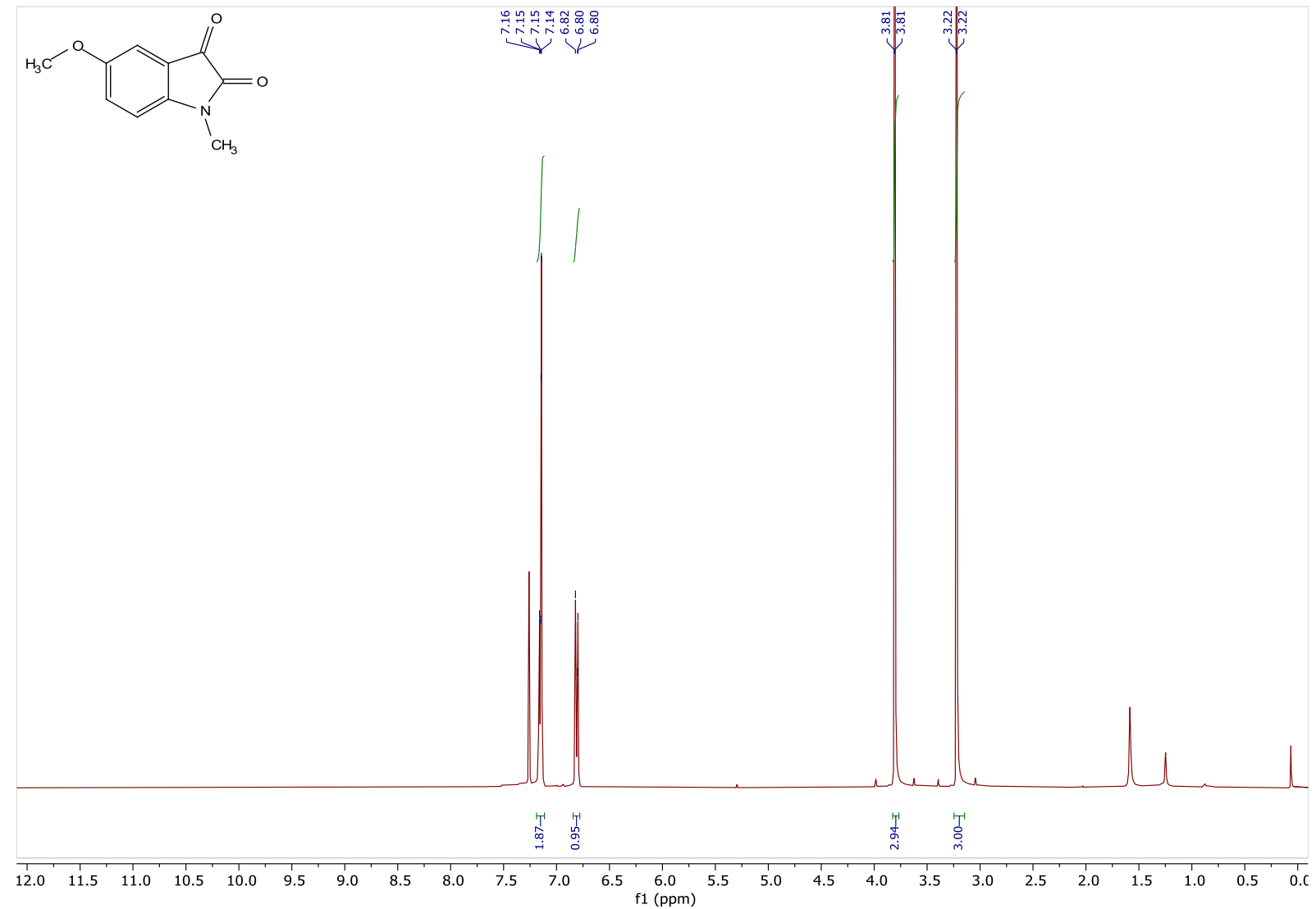

5-fluoro-1-methylindoline-2, 3-dione - ${ }^{1} \mathrm{H}$ NMR (400 MHz, $\left.\mathrm{CDCl}_{3}\right)$

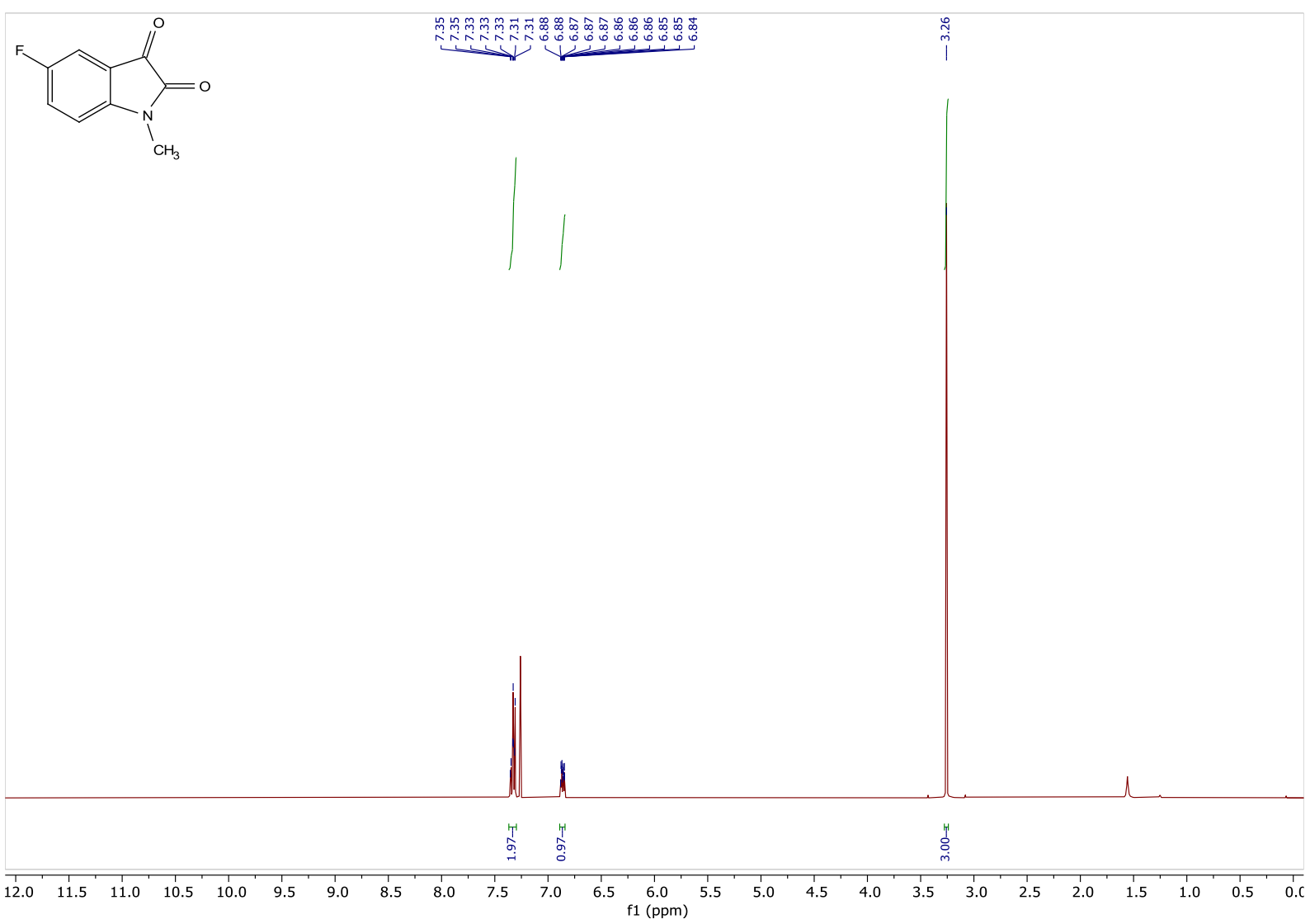


5-chloro-1-methylindoline-2, 3-dione - ${ }^{1} \mathrm{H}$ NMR (400 MHz, $\left.\mathrm{CDCl}_{3}\right)$

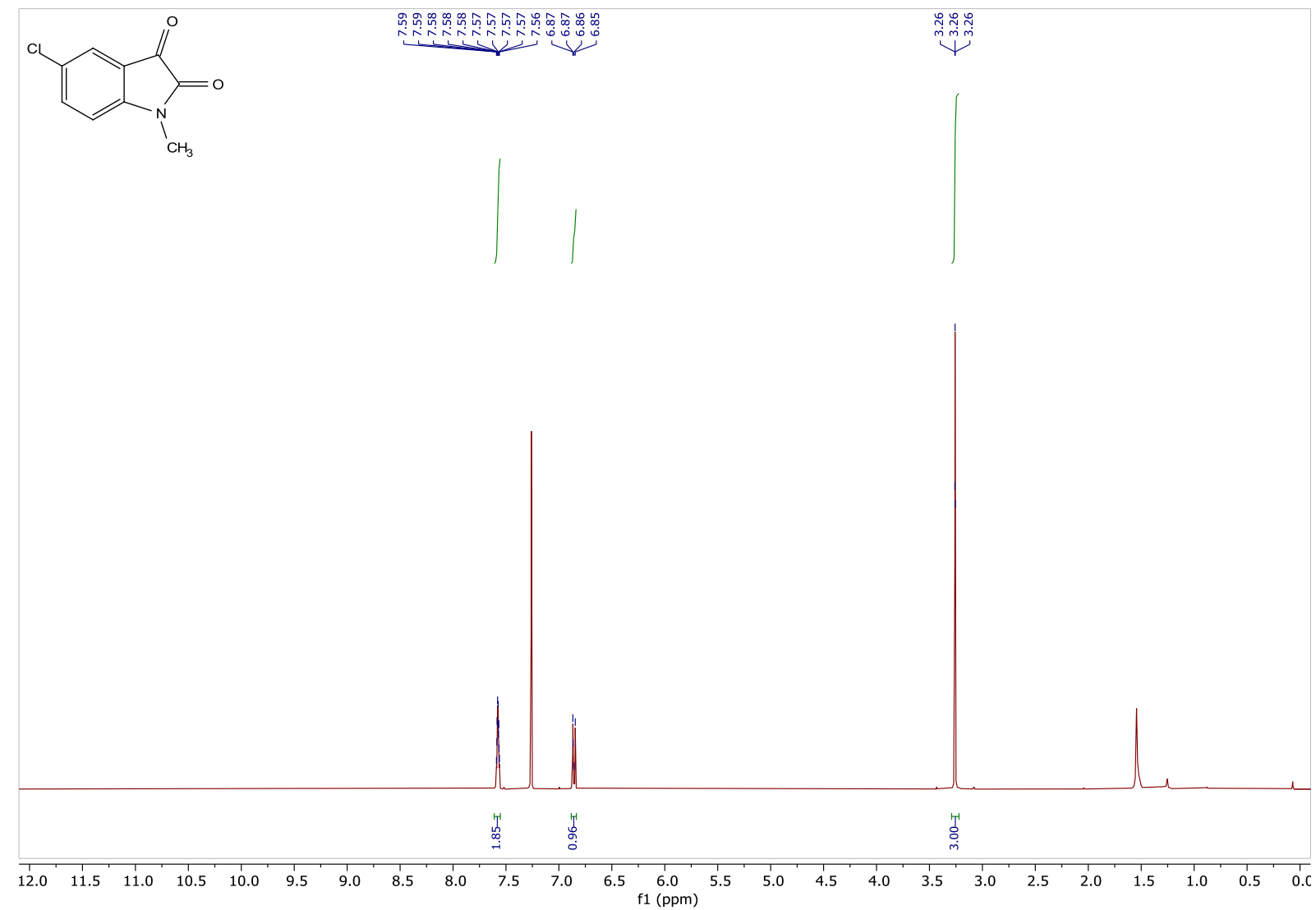

5-bromo-1-methylindoline-2,3-dione - ${ }^{1} \mathrm{H}$ NMR $\left(400 \mathrm{MHz}, \mathrm{CDCl}_{3}\right)$<smiles>CN1C(=O)C(=O)c2cc(Br)ccc21</smiles>
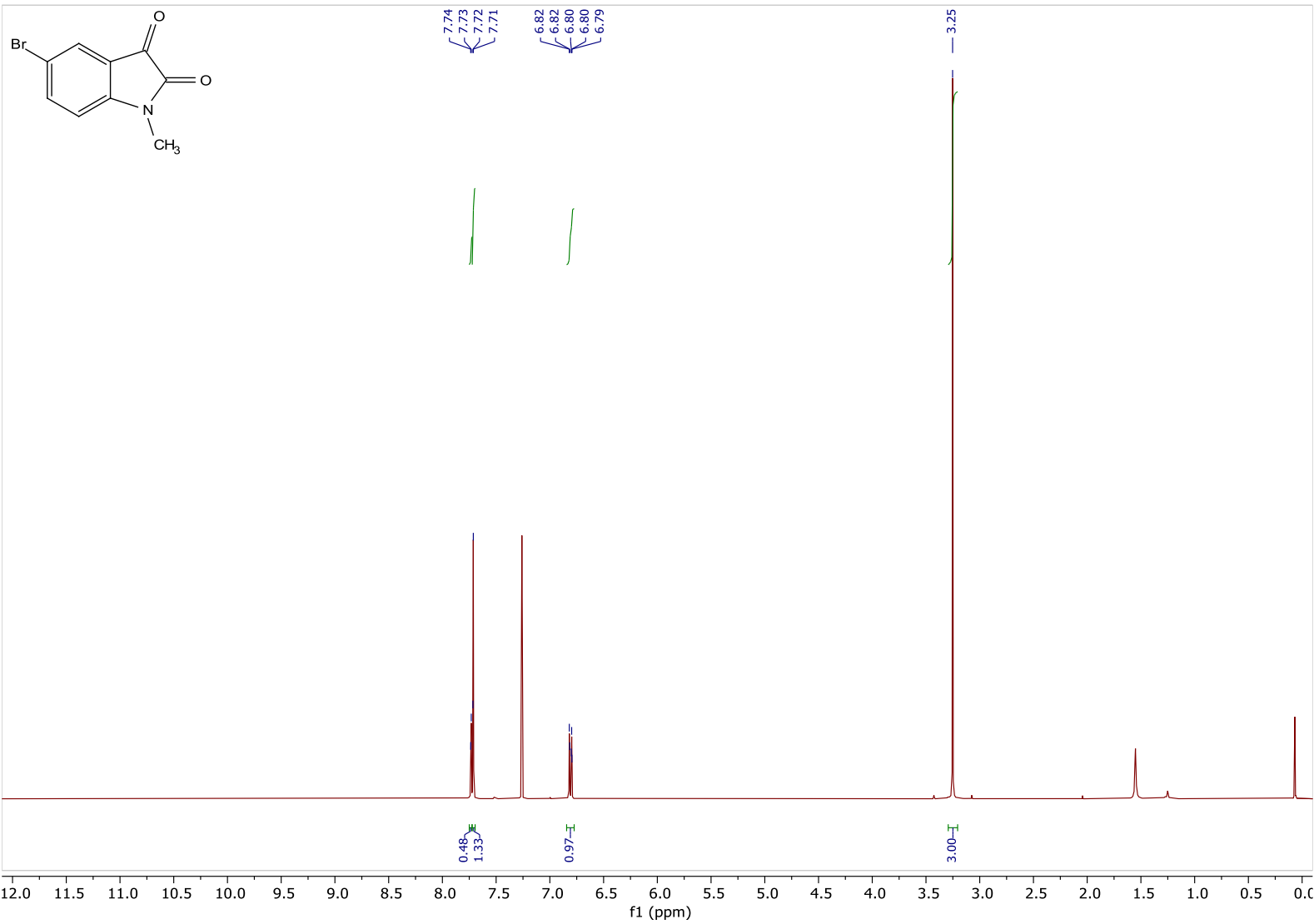
1-benzyl-4,7-dichloroindoline-2,3-dione - ${ }^{1} \mathrm{H}$ NMR $\left(400 \mathrm{MHz}, \mathrm{CDCl}_{3}\right)$

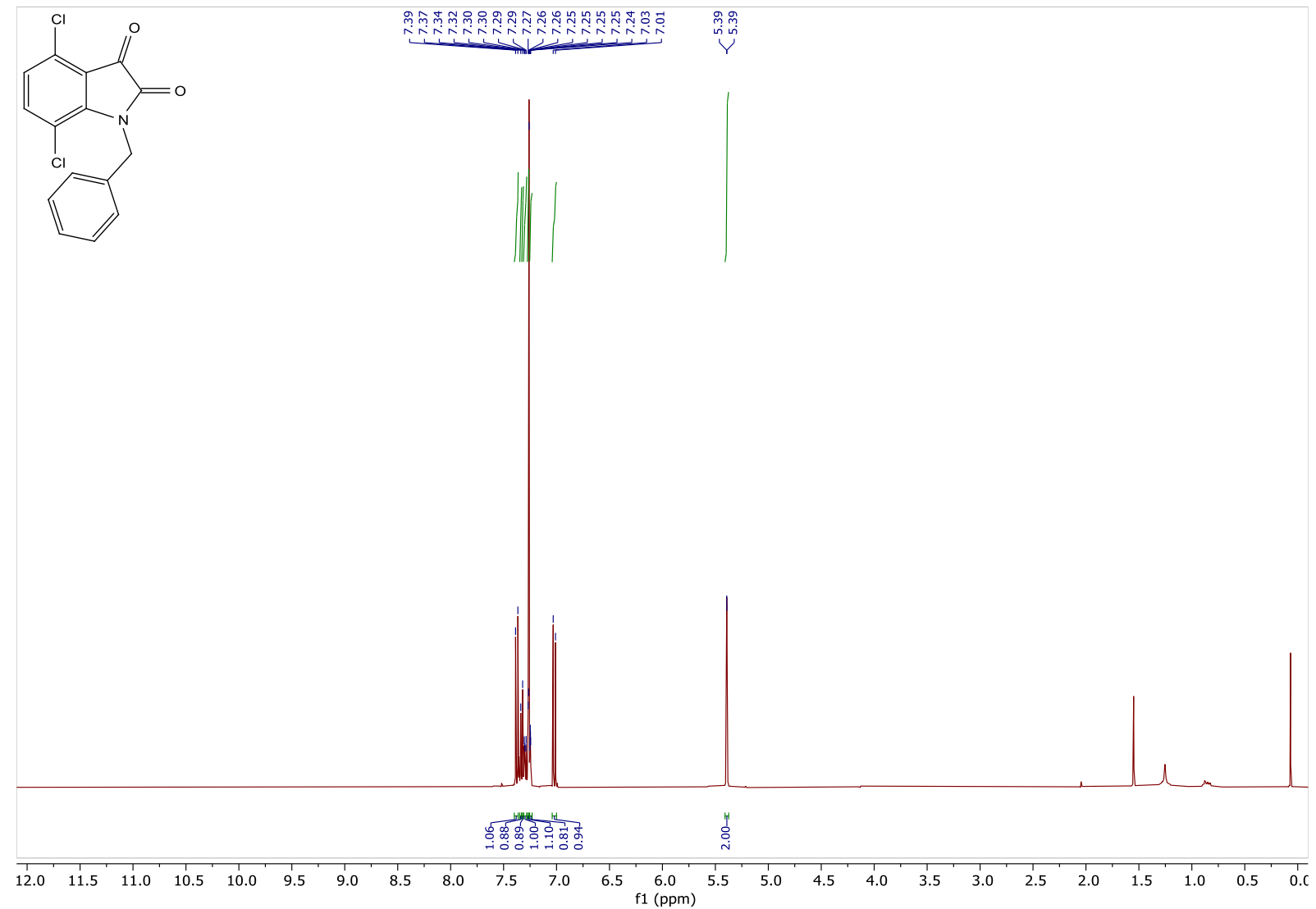

6-chloro-1-methylindoline-2, 3-dione - ${ }^{1} \mathrm{H}$ NMR (400 MHz, $\left.\mathrm{CDCl}_{3}\right)$<smiles>CN1C(=O)C(=O)c2ccc(Cl)cc21</smiles>

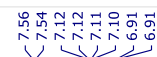

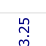

$\mathrm{CH}_{3}$
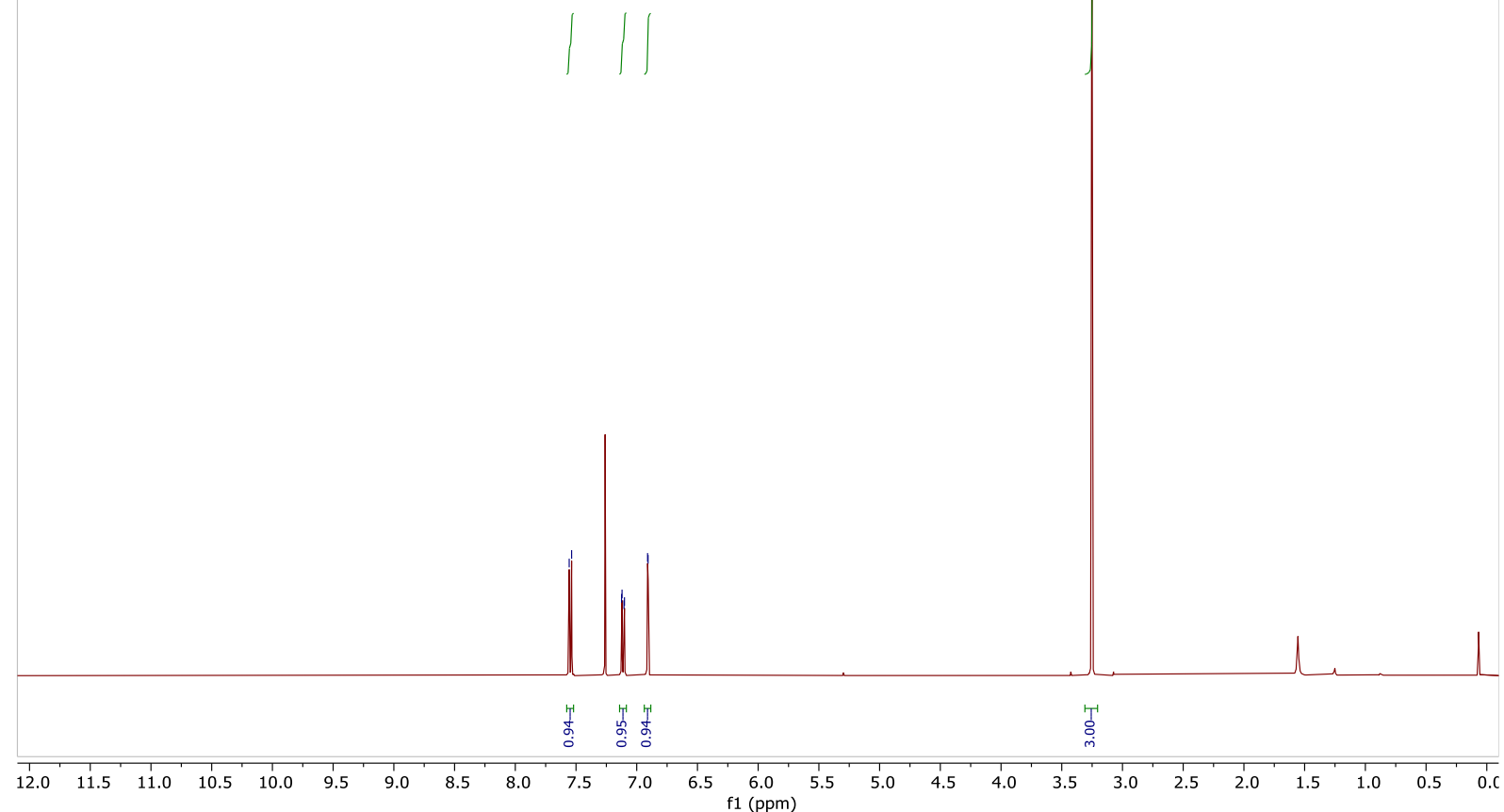
1,5,7-trimethylindoline-2,3-dione - ${ }^{1} \mathrm{H}$ NMR $\left(400 \mathrm{MHz}, \mathrm{CDCl}_{3}\right)$

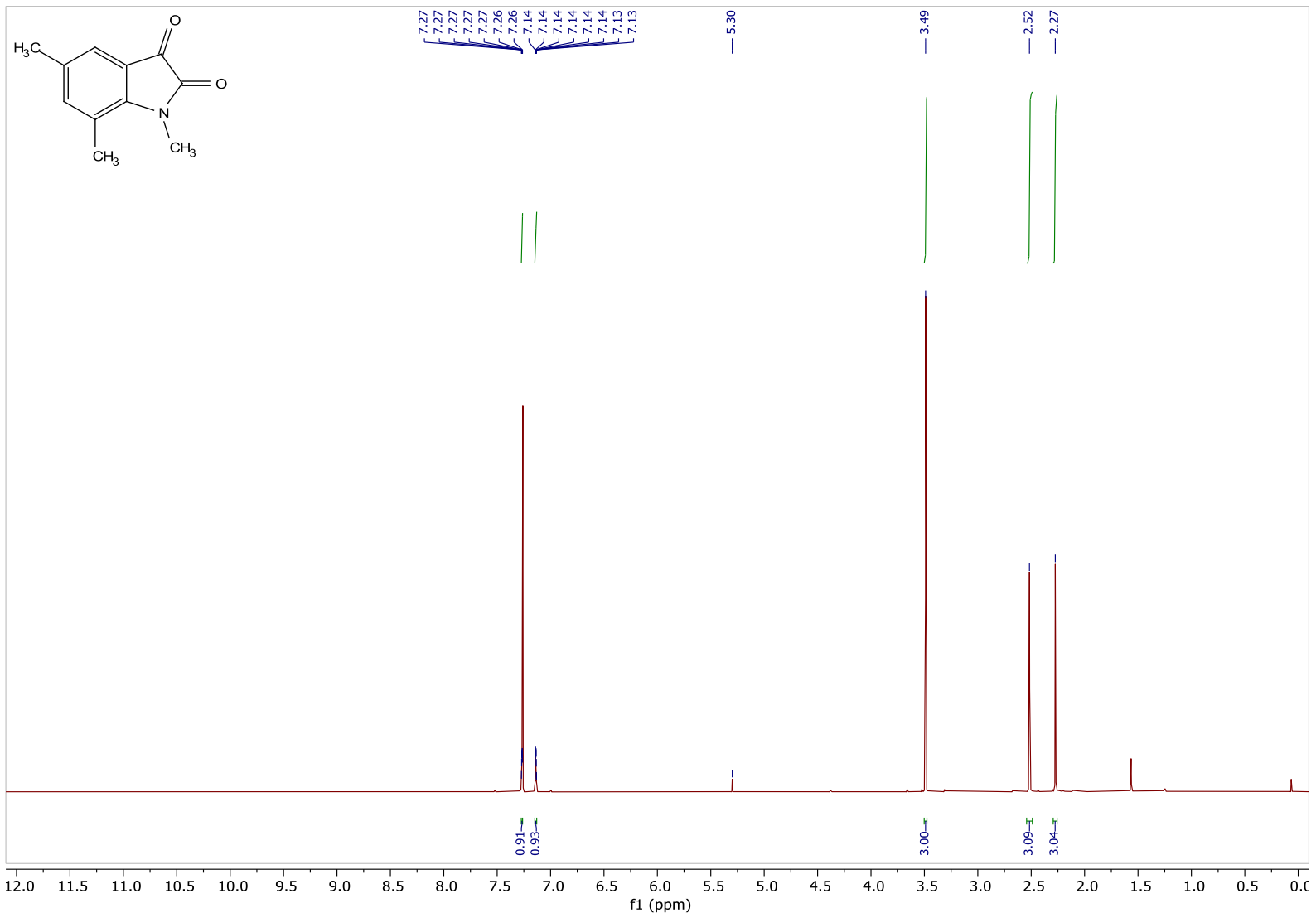




\section{HPLC/SFC Chromatograms}

4a - Chiral HPLC (ADH column, flow rate $=0.5 \mathrm{~mL} / \mathrm{min}, \mathrm{T}=25^{\circ} \mathrm{C}, 35 \% \mathrm{IPA} / n$-hexane, $\mathrm{t}=50$ min): major diastereomer: major at $21.3 \mathrm{~min}$, minor at $17.8 \mathrm{~min}, 92 \%$ ee and minor diastereoisomer. major at $29.2 \mathrm{~min}$, minor at $23.7 \mathrm{~min}, 64 \%$ ee.

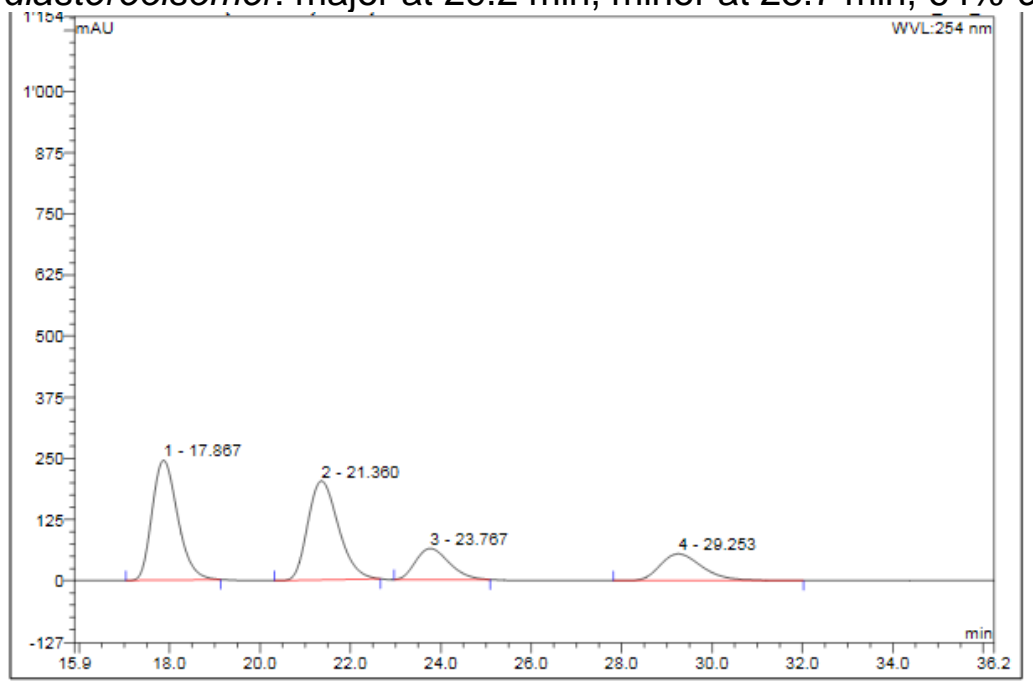

\begin{tabular}{|r|ccrrrrr|}
\hline No. & $\begin{array}{c}\text { Ret.Time } \\
\text { min }\end{array}$ & Peak Name & $\begin{array}{c}\text { Height } \\
\text { mAU }\end{array}$ & $\begin{array}{c}\text { Area } \\
\text { mAU*min }\end{array}$ & $\begin{array}{c}\text { Rel.Area } \\
\%\end{array}$ & Amount & Type \\
\hline 1 & 17.87 & n.a. & 244.964 & 161.041 & 37.16 & n.a. & BMB $^{\star}$ \\
2 & 21.36 & n.a. & 202.022 & 158.301 & 36.53 & n.a. & BMB $^{\star}$ \\
3 & 23.77 & n.a. & 63.587 & 54.888 & 12.66 & n.a. & BMB $^{\star}$ \\
4 & 29.25 & n.a. & 54.646 & 59.167 & 13.65 & n.a. & BMB $^{*}$ \\
\hline Total: & & & 565.220 & 433.397 & 100.00 & 0.000 & \\
\hline
\end{tabular}

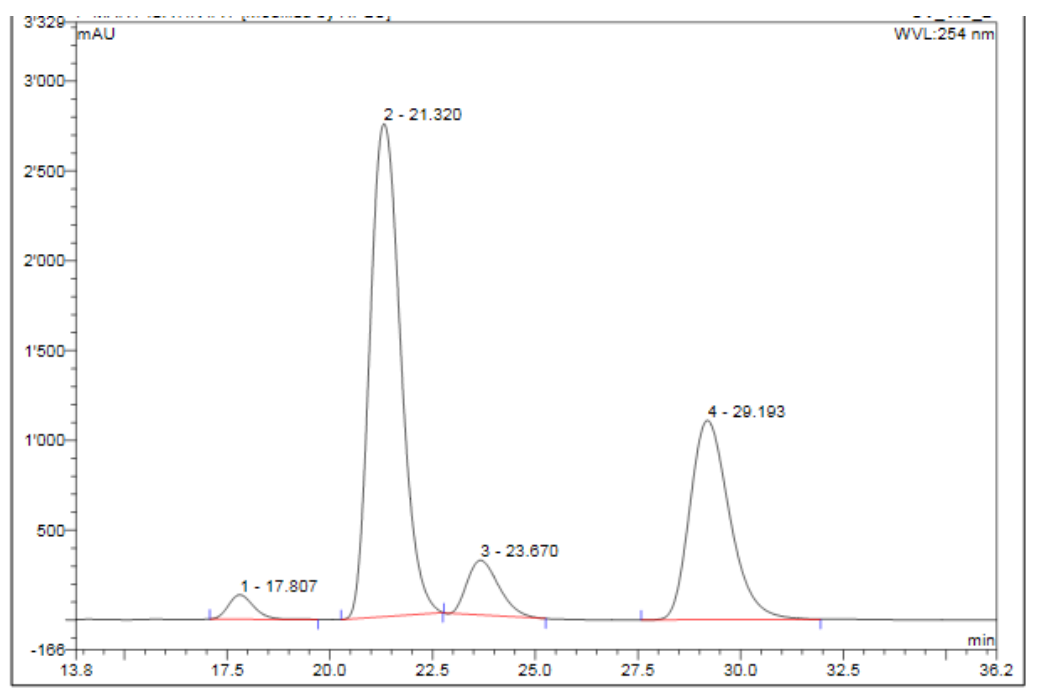

\begin{tabular}{|c|c|c|c|c|c|c|c|}
\hline No. & $\begin{array}{c}\text { Ret.Time } \\
\text { min }\end{array}$ & Peak Name & $\begin{array}{c}\text { Height } \\
\text { mAU }\end{array}$ & $\begin{array}{c}\text { Area } \\
\mathrm{mAU}^{*} \min \end{array}$ & $\begin{array}{c}\text { Rel.Area } \\
\%\end{array}$ & Amount & Type \\
\hline 1 & 17.81 & n.a. & 135.445 & 96.958 & 2.43 & n.a. & $\mathrm{BMB}^{\star}$ \\
\hline 2 & 21.32 & n.a. & 2744.236 & 2372.839 & 59.51 & n.a. & $\mathrm{BMB}^{\star}$ \\
\hline 3 & 23.67 & n.a. & 304.070 & 273.058 & 6.85 & n.a. & $\mathrm{BMB}^{*}$ \\
\hline 4 & 29.19 & n.a. & 1109.149 & 1244.587 & 31.21 & n.a. & $\mathrm{BMB}^{*}$ \\
\hline Total: & & & 4292.899 & 3987.443 & 100.00 & 0.000 & \\
\hline
\end{tabular}


4b - Chiral SFC (Whelk column, flow rate $=2 \mathrm{~mL} / \mathrm{min}, \mathrm{T}=40^{\circ} \mathrm{C}, 15 \%$ methanol $/ \mathrm{CO}_{2}, \mathrm{t}=15 \mathrm{~min}$ ): major diastereomer: major at $10.0 \mathrm{~min}$, minor at $12.5 \mathrm{~min}, 98 \%$ ee and minor diastereoisomer: major at $11.2 \mathrm{~min}$, minor at $13.8 \mathrm{~min}, 48 \%$ ee.

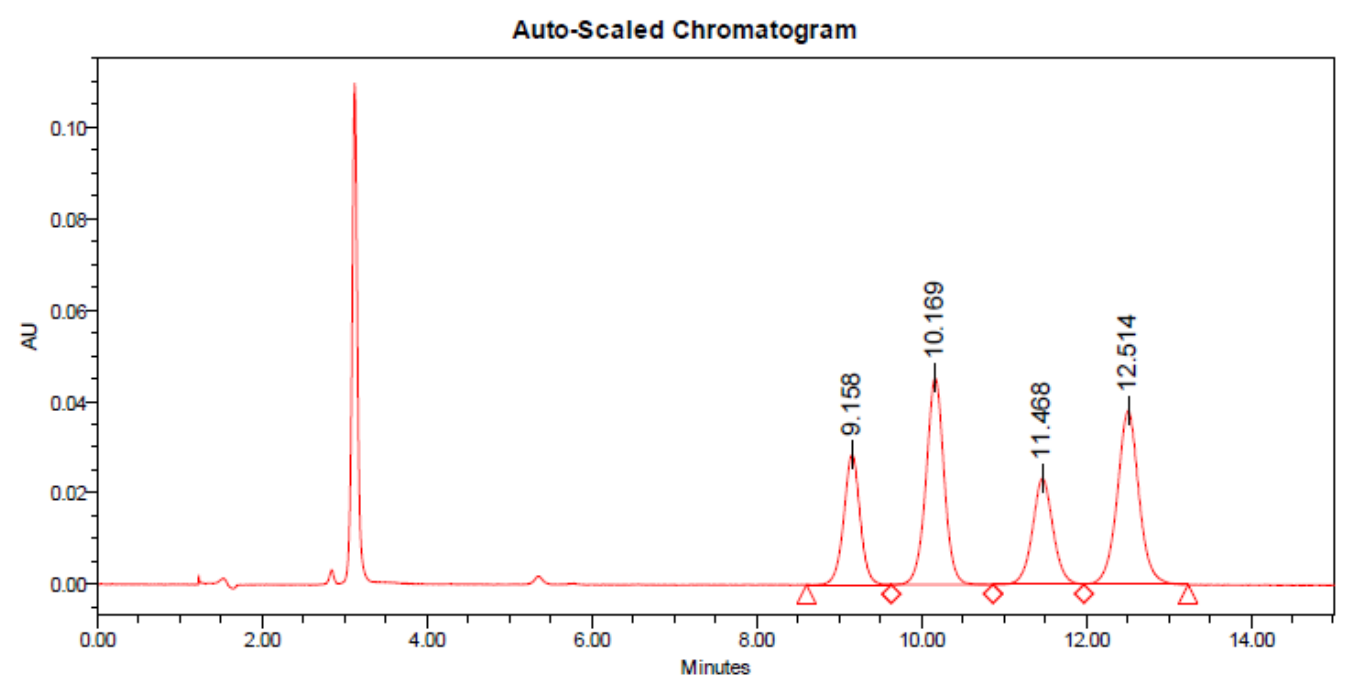

Processed Channel: PDA Ch1 254nm@1.2nm

-Compens.

\begin{tabular}{|l|l|r|r|r|r|}
\hline & Processed Channel & $\begin{array}{c}\text { Retention } \\
\text { Time ( }(\mathbf{m i n})\end{array}$ & Area & $\%$ Area & Height \\
\hline 1 & PDA Ch1 254nm@1.2nm -Compens. & 9.158 & 392491 & 17.92 & 28476 \\
\hline 2 & PDA Ch1 254nm@1.2nm -Compens. & 10.169 & 691322 & 31.57 & 45227 \\
\hline 3 & PDA Ch1 254nm@1.2nm -Compens. & 11.468 & 405815 & 18.53 & 23270 \\
\hline 4 & PDA Ch1 254nm@1.2nm -Compens. & 12514 & 700394 & 31.98 & 38025 \\
\hline
\end{tabular}

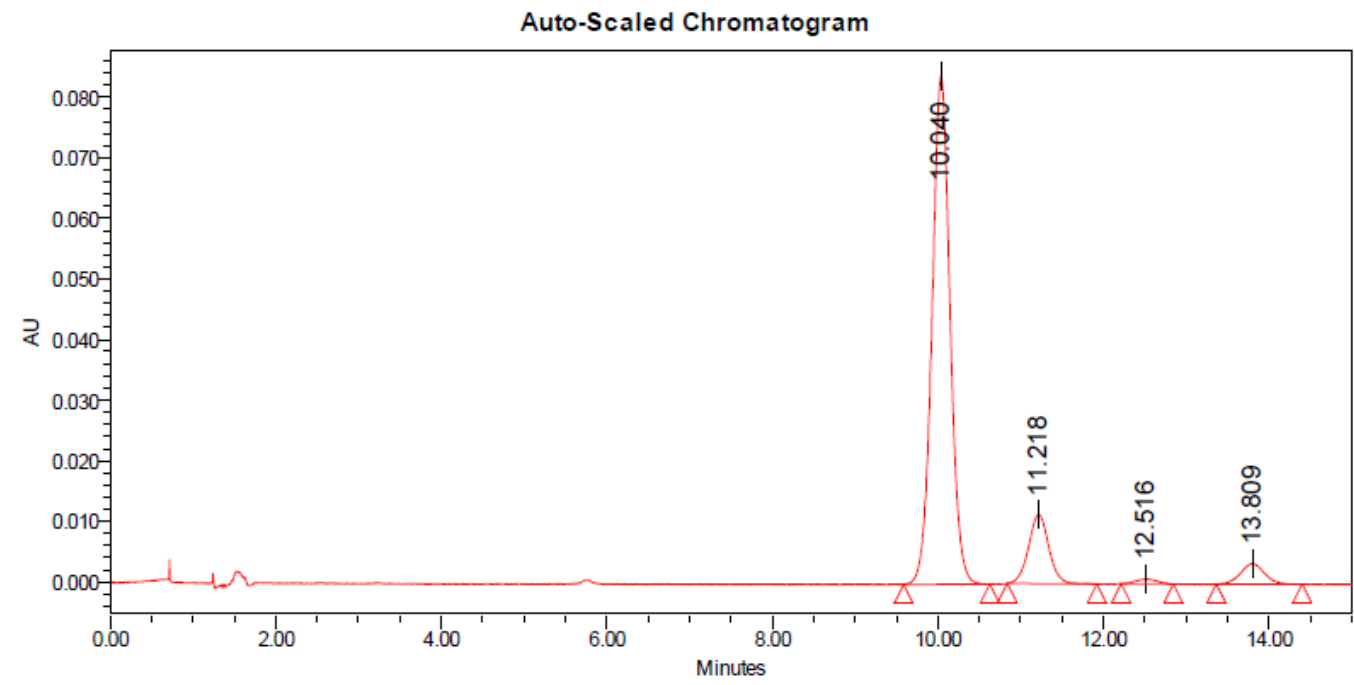

Processed Channel: PDA Ch1 254nm@1.2nm -Compens.

\begin{tabular}{|l|l|r|r|r|r|}
\hline & Processed Channel & $\begin{array}{c}\text { Retention } \\
\text { Time }(\min )\end{array}$ & Area & $\%$ Area & Height \\
\hline 1 & PDA Ch1 254nm@1.2nm -Compens. & 10.040 & 1266865 & 82.23 & 83854 \\
\hline 2 & PDA Ch1 254nm@1.2nm -Compens. & 11.218 & 193017 & 12.53 & 11403 \\
\hline 3 & PDA Ch1 254nm@1.2nm-Compens. & 12.516 & 13810 & 0.90 & 828 \\
\hline 4 & PDA Ch1 254nm@1.2nm-Compens. & 13.809 & 67023 & 4.35 & 3386 \\
\hline
\end{tabular}


4c - Chiral SFC (Whelk column, flow rate $=2 \mathrm{~mL} / \mathrm{min}, \mathrm{T}=40^{\circ} \mathrm{C}, 8 \% \mathrm{IPA} / \mathrm{CO}_{2}, \mathrm{t}=75 \mathrm{~min}$ ): major diastereomer. major at $34.6 \mathrm{~min}$, minor at $32.3 \mathrm{~min}, 93 \%$ ee and minor diastereoisomer. major at $37.0 \mathrm{~min}$, minor at $69.4 \mathrm{~min}, 48 \%$ ee.

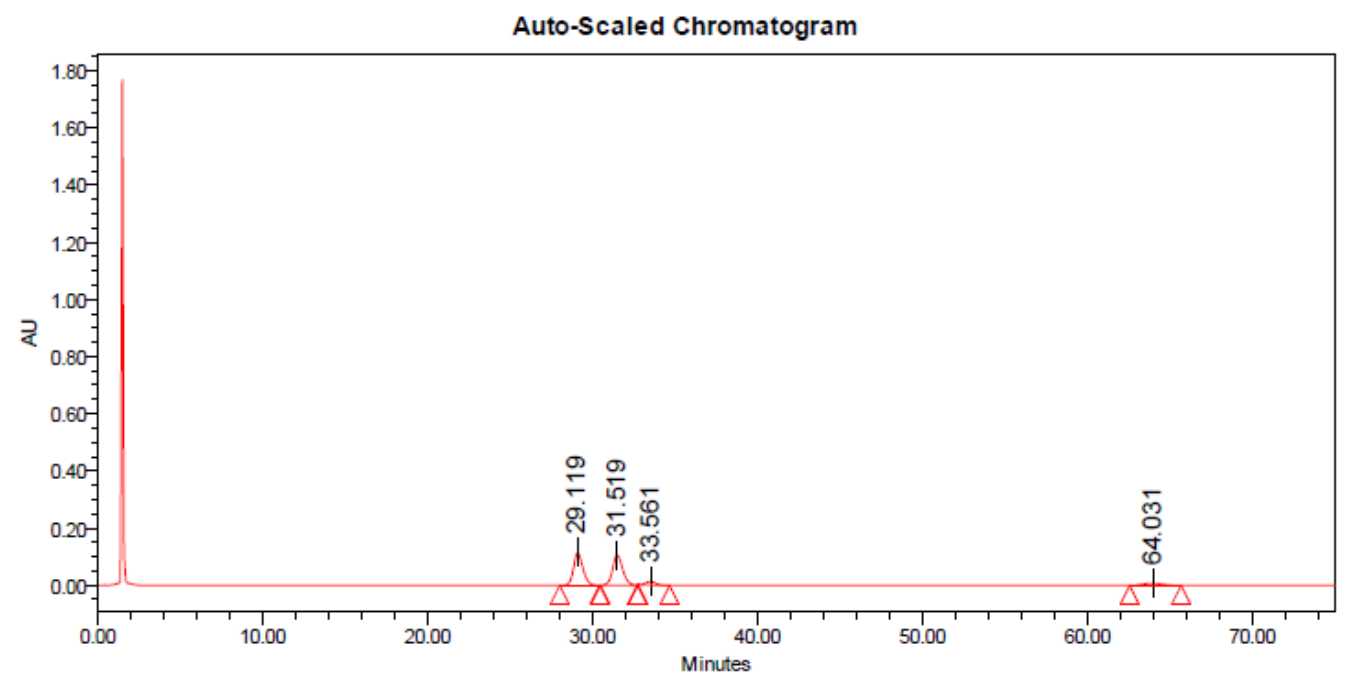

Processed Channel: PDA Ch1 254nm@1.2nm

-Compens.

\begin{tabular}{|l|l|r|r|r|r|}
\hline & Processed Channel & $\begin{array}{r}\text { Retention } \\
\text { Time ( } \min )\end{array}$ & Area & \% Area & Height \\
\hline 1 & PDA Ch1 254nm@1.2nm -Compens. & 29.119 & 4528257 & 44.93 & 114578 \\
\hline 2 & PDA Ch1 254nm@1.2nm -Compens. & 31.519 & 4451811 & 44.17 & 104584 \\
\hline 3 & PDA Ch1 254nm@1.2nm -Compens. & 33.561 & 546411 & 5.42 & 13011 \\
\hline 4 & PDA Ch1 254nm@1.2nm -Compens. & 64.031 & 552593 & 5.48 & 7047 \\
\hline
\end{tabular}

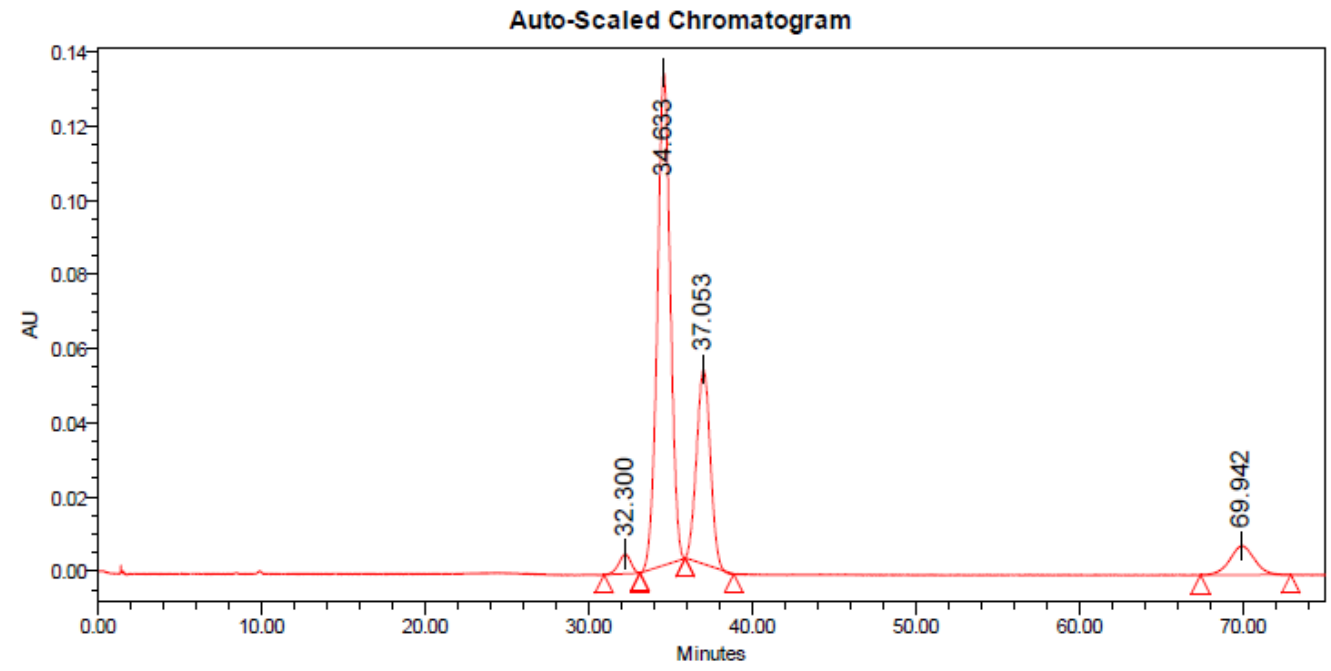

Processed Channel: PDA Ch1 254nm@1.2nm

-Compens.

\begin{tabular}{|l|l|r|r|r|r|}
\hline & Processed Channel & $\begin{array}{c}\text { Retention } \\
\text { Time ( } \min \text { ) }\end{array}$ & Area & $\%$ Area & Height \\
\hline 1 & PDA Ch1 254nm@1.2nm -Compens. & 32.300 & 268076 & 2.33 & 5261 \\
\hline 2 & PDA Ch1 254nm@1.2nm -Compens. & 34.633 & 7334805 & 63.79 & 132713 \\
\hline 3 & PDA Ch1 254nm@1.2nm -Compens. & 37.053 & 3074344 & 26.74 & 52467 \\
\hline 4 & PDA Ch1 254nm@1.2nm -Compens. & 69.942 & 820400 & 7.14 & 7983 \\
\hline
\end{tabular}


4d - Chiral SFC (AS3 column, flow rate $=2 \mathrm{~mL} / \mathrm{min}, \mathrm{T}=40{ }^{\circ} \mathrm{C}, 5 \%$ methanol $/ \mathrm{CO}_{2}, \mathrm{t}=20 \mathrm{~min}$ ): major diastereomer: major at $11.4 \mathrm{~min}$, minor at $5.2 \mathrm{~min}, 92 \%$ ee and minor diastereoisomer. major at $7.0 \mathrm{~min}$, minor at $9.0 \mathrm{~min}, 48 \%$ ee.

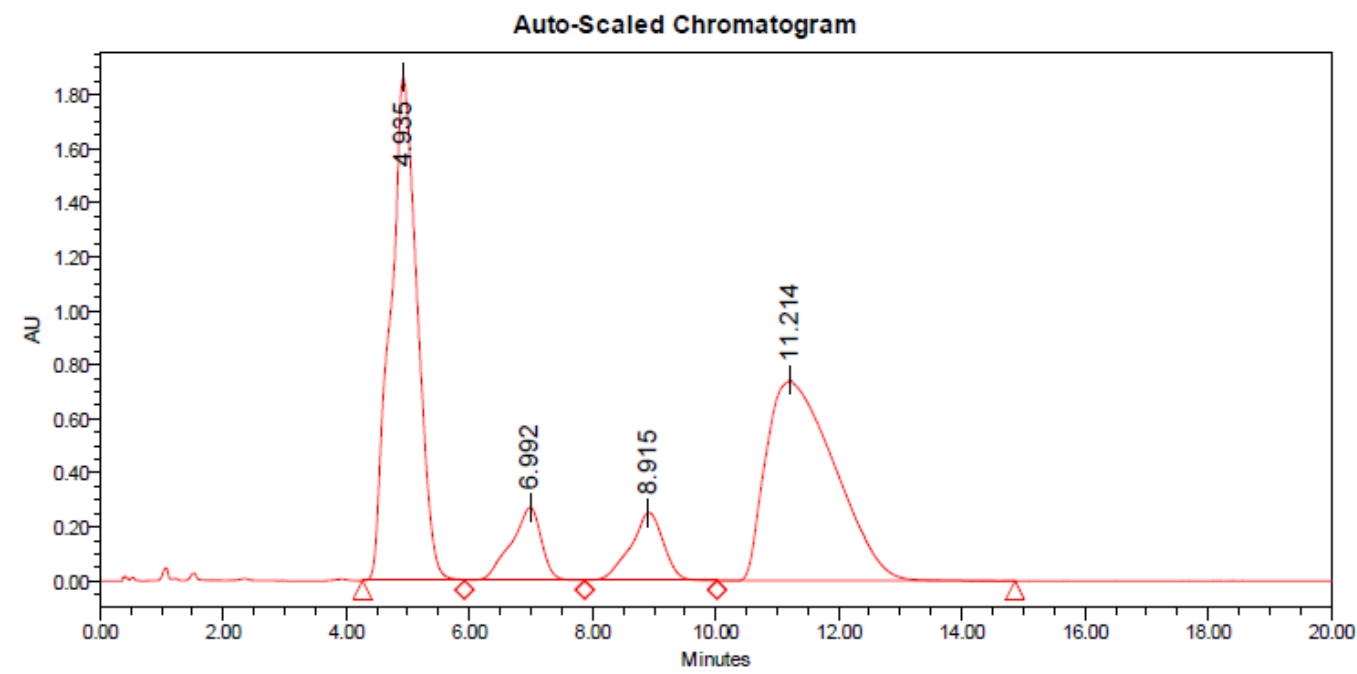

Processed Channel: PDA Ch2 214nm@1.2nm

\begin{tabular}{|l|c|r|r|r|r|}
\hline & Processed Channel & $\begin{array}{r}\text { Retention } \\
\text { Time }(\min )\end{array}$ & Area & $\%$ Area & Height \\
\hline 1 & PDA Ch2 214nm@1.2nm & 4.935 & 56619972 & 42.75 & 1859161 \\
\hline 2 & PDA Ch2 214nm@1.2nm & 6.992 & 9358544 & 7.07 & 272217 \\
\hline 3 & PDA Ch2 214nm@1.2nm & 8.915 & 9438349 & 7.13 & 252115 \\
\hline 4 & PDA Ch2 214nm@1.2nm & 11.214 & 57026598 & 43.06 & 743602 \\
\hline
\end{tabular}

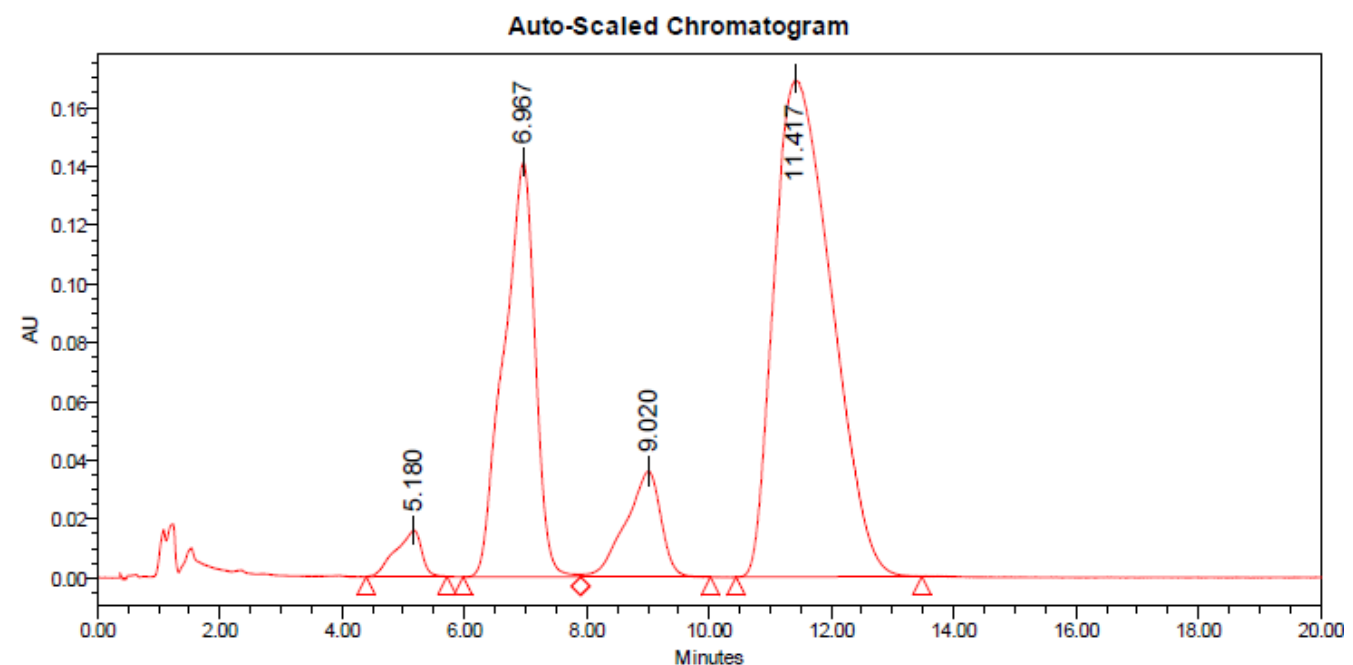

Processed Channel: PDA Ch1 254nm@1.2nm

-Compens.

\begin{tabular}{|l|l|r|r|r|r|}
\hline & Processed Channel & $\begin{array}{c}\text { Retention } \\
\text { Time }(\mathrm{min})\end{array}$ & Area & $\%$ Area & Height \\
\hline 1 & PDA Ch1 254nm@1.2nm-Compens. & 5.180 & 463506 & 2.59 & 15742 \\
\hline 2 & PDA Ch1 254nm@1.2nm-Compens. & 6.967 & 5009613 & 27.96 & 141104 \\
\hline 3 & PDA Ch1 254nm@1.2nm-Compens. & 9.020 & 1432588 & 8.00 & 36033 \\
\hline 4 & PDA Ch1 254nm@1.2nm-Compens. & 11.417 & 11010467 & 61.46 & 169280 \\
\hline
\end{tabular}


4e - Chiral SFC (AD3 column, flow rate $=2 \mathrm{~mL} / \mathrm{min}, \mathrm{T}=40{ }^{\circ} \mathrm{C}, 15 \%$ methanol $/ \mathrm{CO}_{2}, \mathrm{t}=25 \mathrm{~min}$ ): major diastereomer: major at $7.0 \mathrm{~min}$, minor at $14.7 \mathrm{~min}, 99 \%$ ee.

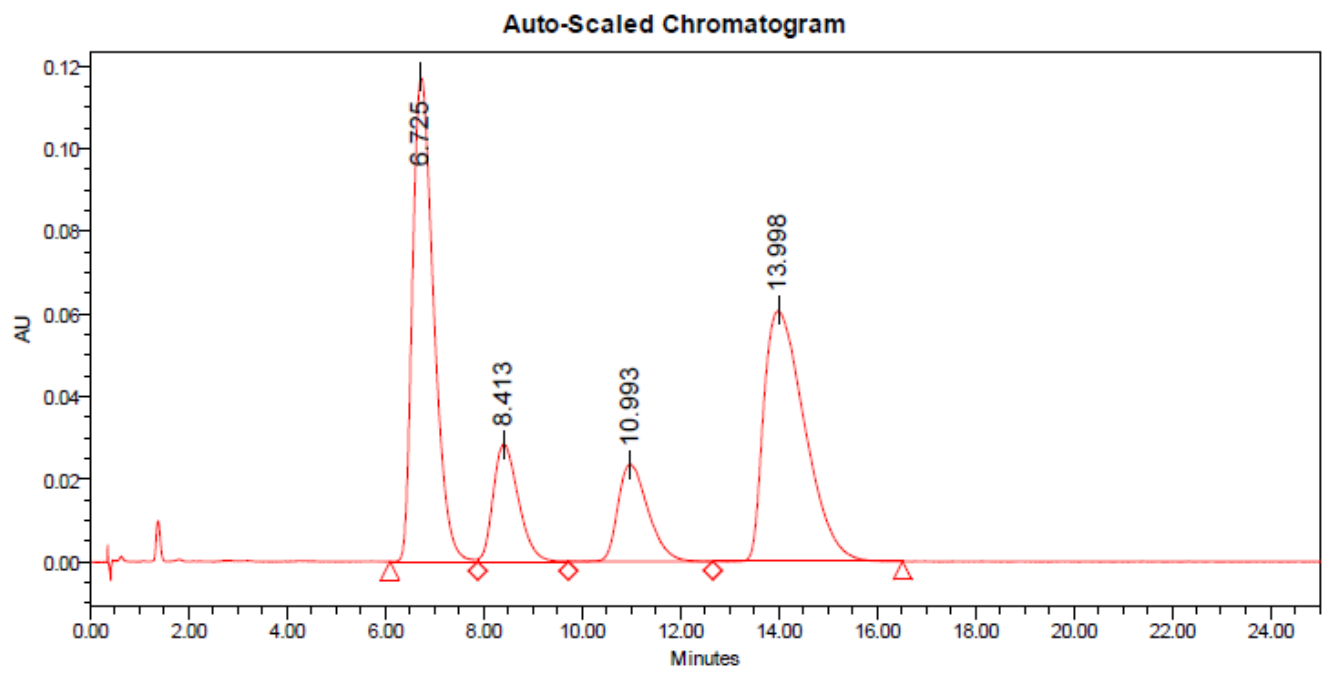

Processed Channel: PDA Ch1 254nm@1.2nm

-Compens.

\begin{tabular}{|c|c|r|r|r|r|}
\hline & Processed Channel & $\begin{array}{r}\text { Retention } \\
\text { Time }(\mathrm{min})\end{array}$ & Area & $\%$ Area & Height \\
\hline 1 & PDA Ch1 254nm@1.2nm -Compens. & 6.725 & 3502666 & 38.88 & 117089 \\
\hline 2 & PDA Ch1 254nm@1.2nm -Compens. & 8.413 & 1005929 & 11.17 & 28376 \\
\hline 3 & PDA Ch1 254nm@1.2nm -Compens. & 10.993 & 1002608 & 11.13 & 23521 \\
\hline 4 & PDA Ch1 254nm@1.2nm -Compens. & 13.998 & 3497989 & 38.83 & 60749 \\
\hline
\end{tabular}

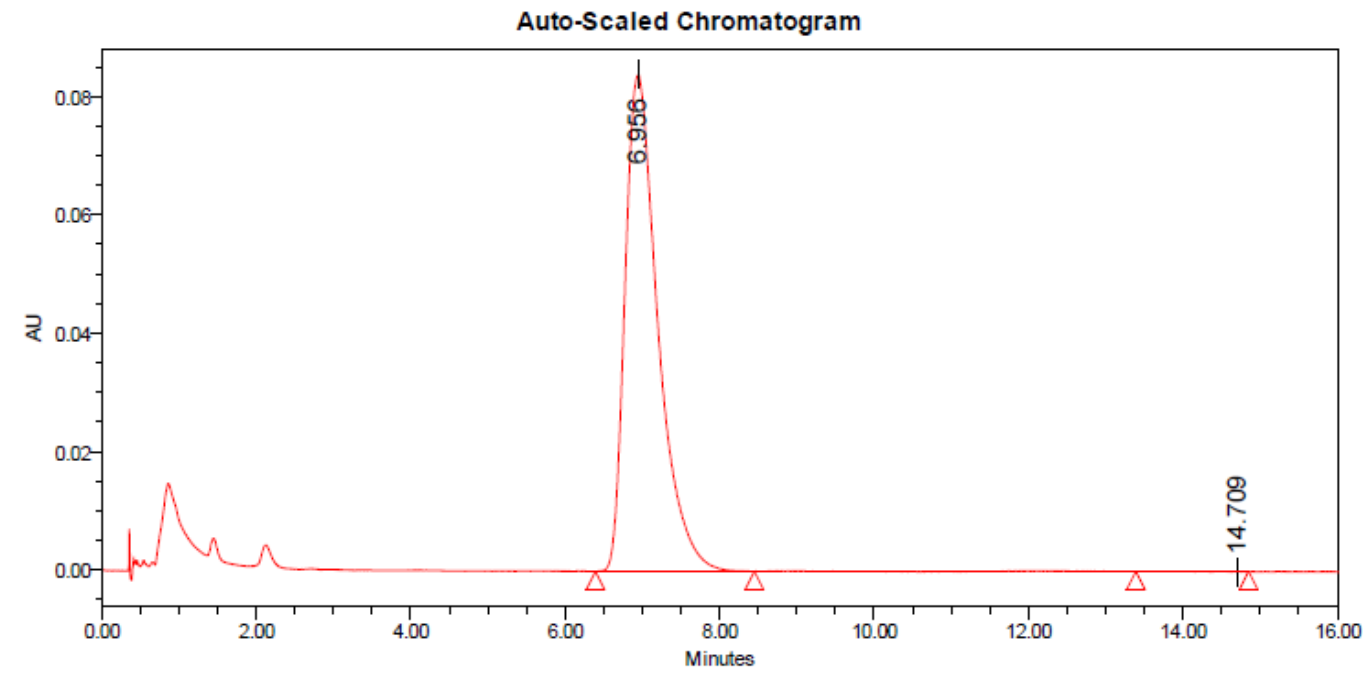

Processed Channel: PDA Ch1 254nm@1.2nm

-Compens.

\begin{tabular}{|l|c|r|r|r|r|}
\hline & Processed Channel & $\begin{array}{c}\text { Retention } \\
\text { Time (min) }\end{array}$ & Area & \% Area & Height \\
\hline 1 & PDA Ch1 254nm@1.2nm -Compens. & 6.956 & 2480338 & 99.94 & 83886 \\
\hline 2 & PDA Ch1 254nm@1.2nm -Compens. & 14.709 & 1500 & 0.06 & -84 \\
\hline
\end{tabular}


4f - Chiral SFC (OJ3 column, flow rate $=2 \mathrm{~mL} / \mathrm{min}, \mathrm{T}=40{ }^{\circ} \mathrm{C}, 10 \%$ methanol $/ \mathrm{CO}_{2}, \mathrm{t}=10 \mathrm{~min}$ ): major diastereomer: major at $2.0 \mathrm{~min}$, minor at $2.8 \mathrm{~min}, 89 \%$ ee and minor diastereoisomer. major at $2.4 \mathrm{~min}$, minor at $4.6 \mathrm{~min}, 56 \%$ ee.

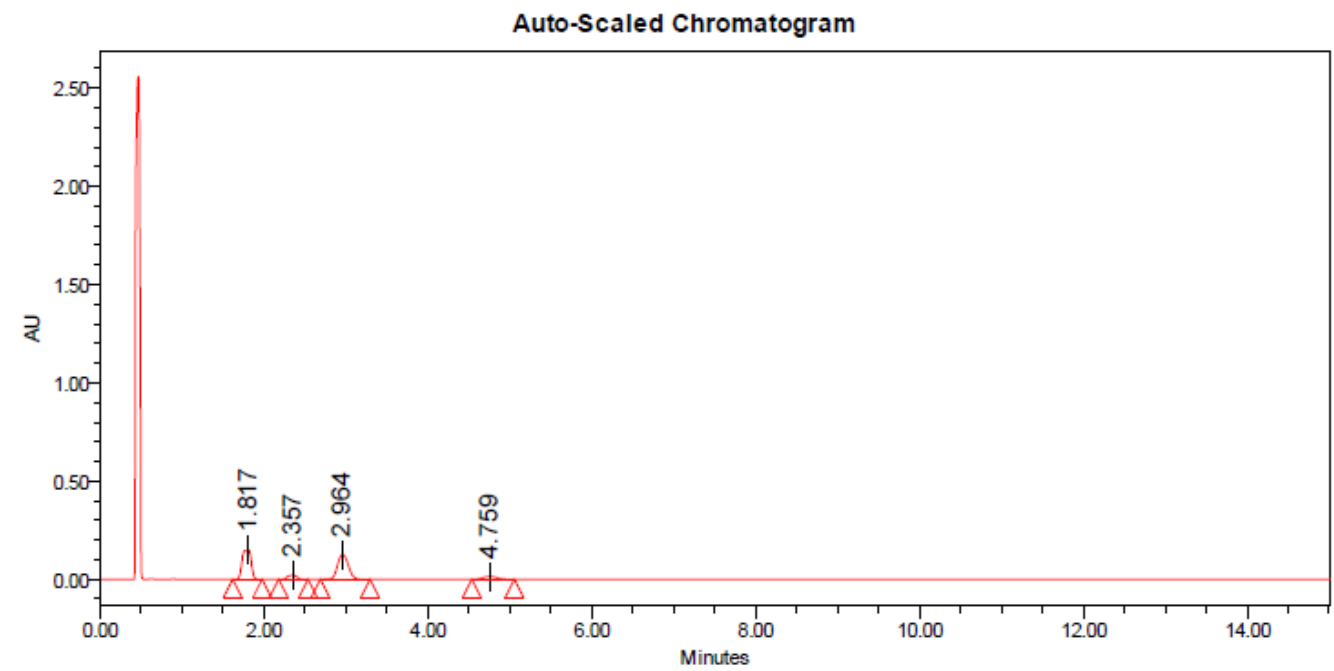

Processed Channel: PDA Ch1 254nm@1.2nm -Compens.

\begin{tabular}{|l|l|r|r|r|r|}
\hline & Processed Channel & $\begin{array}{r}\text { Retention } \\
\text { Time }(\boldsymbol{m i n})\end{array}$ & Area & $\%$ Area & Height \\
\hline 1 & PDA Ch1 254nm@1.2nm -Compens. & 1.817 & 1131223 & 43.80 & 148723 \\
\hline 2 & PDA Ch1 254nm@1.2nm -Compens. & 2.357 & 156242 & 6.05 & 20131 \\
\hline 3 & PDA Ch1 254nm@1.2nm -Compens. & 2.964 & 1140317 & 44.15 & 125519 \\
\hline 4 & PDA Ch1 254nm@1.2nm -Compens. & 4.759 & 154785 & 5.99 & 13738 \\
\hline
\end{tabular}

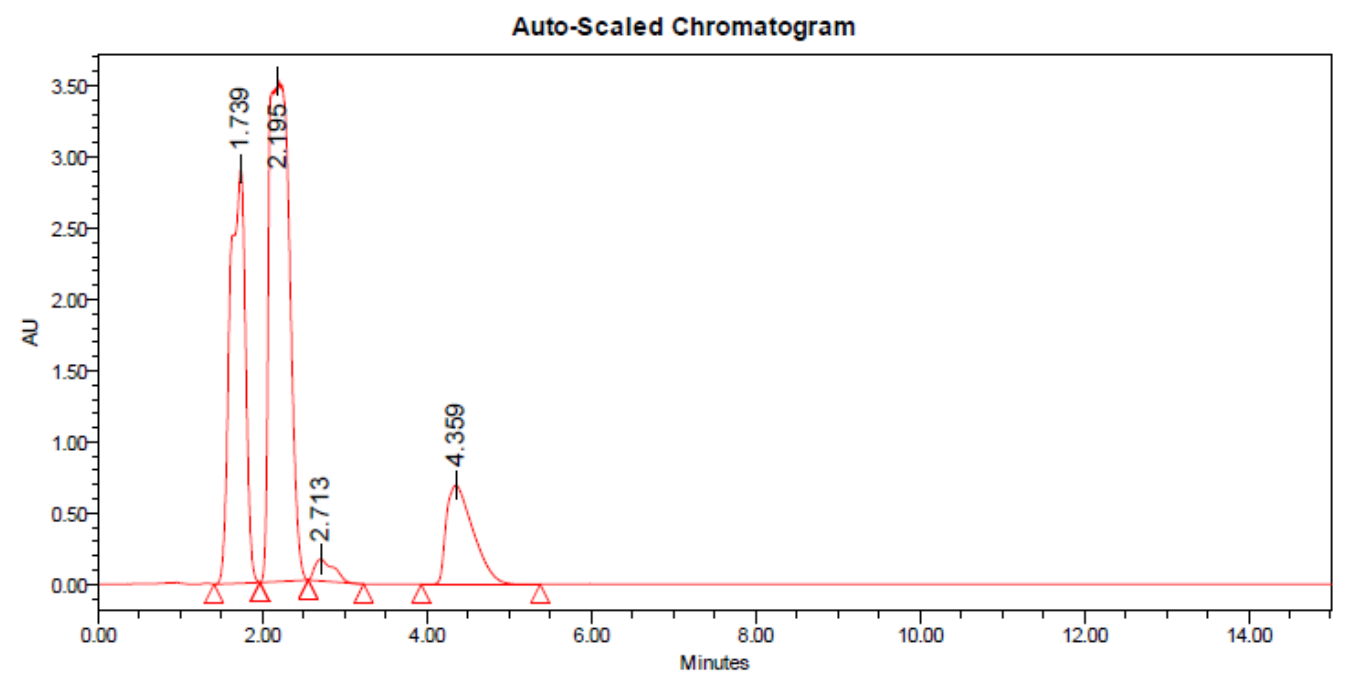

Processed Channel: PDA Ch1 254nm@1.2nm -Compens.

\begin{tabular}{|l|l|r|r|r|r|}
\hline & Processed Channel & $\begin{array}{r}\text { Retention } \\
\text { Time ( } \min )\end{array}$ & Area & $\%$ Area & Height \\
\hline 1 & PDA Ch1 254nm@1.2nm -Compens. & 1.739 & 37770786 & 32.17 & 2910114 \\
\hline 2 & PDA Ch1 254nm@1.2nm -Compens. & 2.195 & 61420335 & 52.31 & 3518369 \\
\hline 3 & PDA Ch1 254nm@1.2nm -Compens. & 2.713 & 2482380 & 2.11 & 152660 \\
\hline 4 & PDA Ch1 254nm@1.2nm -Compens. & 4.359 & 15747208 & 13.41 & 694775 \\
\hline
\end{tabular}


4 g - Chiral SFC (Whelk column, flow rate $=2 \mathrm{~mL} / \mathrm{min}, \mathrm{T}=40^{\circ} \mathrm{C}, 15 \% \mathrm{IPA} / \mathrm{CO}_{2}, \mathrm{t}=25 \mathrm{~min}$ ): major diastereomer. major at $11.4 \mathrm{~min}$, minor at $12.4 \mathrm{~min}, 88 \%$ ee and minor diastereoisomer. major at $13.3 \mathrm{~min}$, minor at $18.5 \mathrm{~min}, 67 \%$ ee.

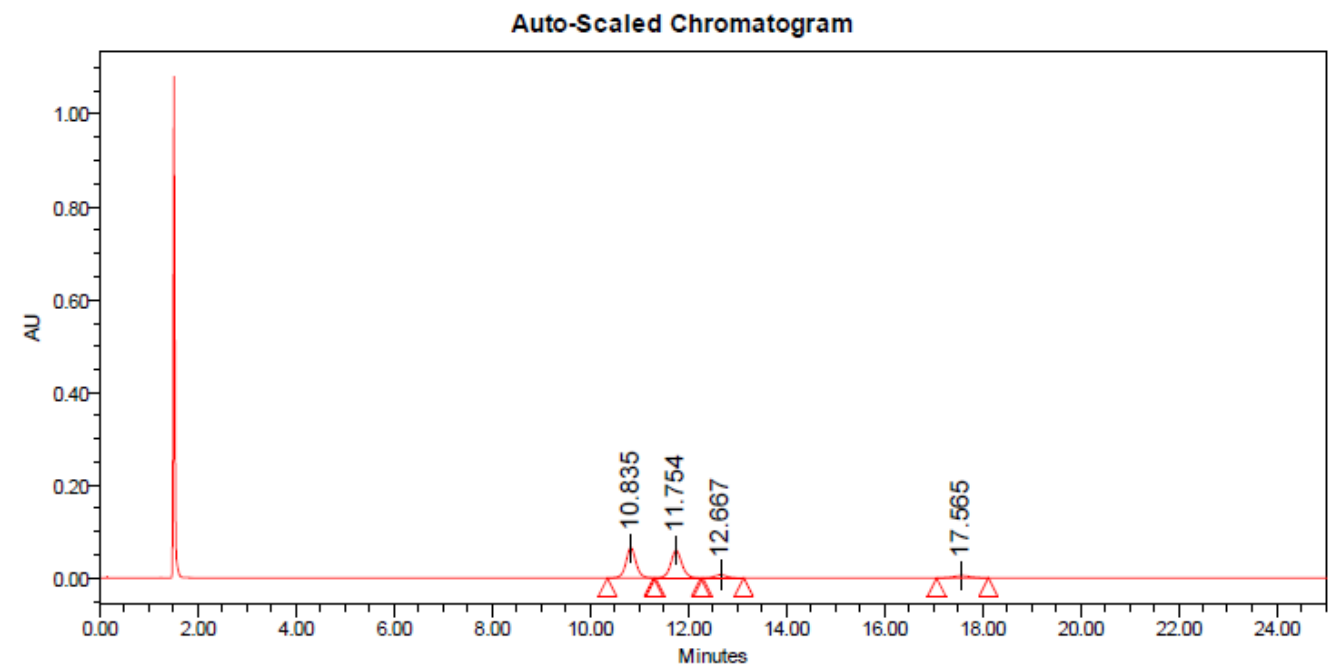

Processed Channel: PDA Ch1 254nm@1.2nm

-Compens.

\begin{tabular}{|l|l|r|r|r|r|}
\hline & Processed Channel & $\begin{array}{c}\text { Retention } \\
\text { Time }(\boldsymbol{m i n})\end{array}$ & Area & \% Area & Height \\
\hline 1 & PDA Ch1 254nm@1.2nm -Compens. & 10.835 & 922414 & 45.13 & 64949 \\
\hline 2 & PDA Ch1 254nm@1.2nm -Compens. & 11.754 & 912734 & 44.66 & 58547 \\
\hline 3 & PDA Ch1 254nm@1.2nm -Compens. & 12667 & 103739 & 5.08 & 6396 \\
\hline 4 & PDA Ch1 254nm@1.2nm -Compens. & 17.565 & 104832 & 5.13 & 4574 \\
\hline
\end{tabular}

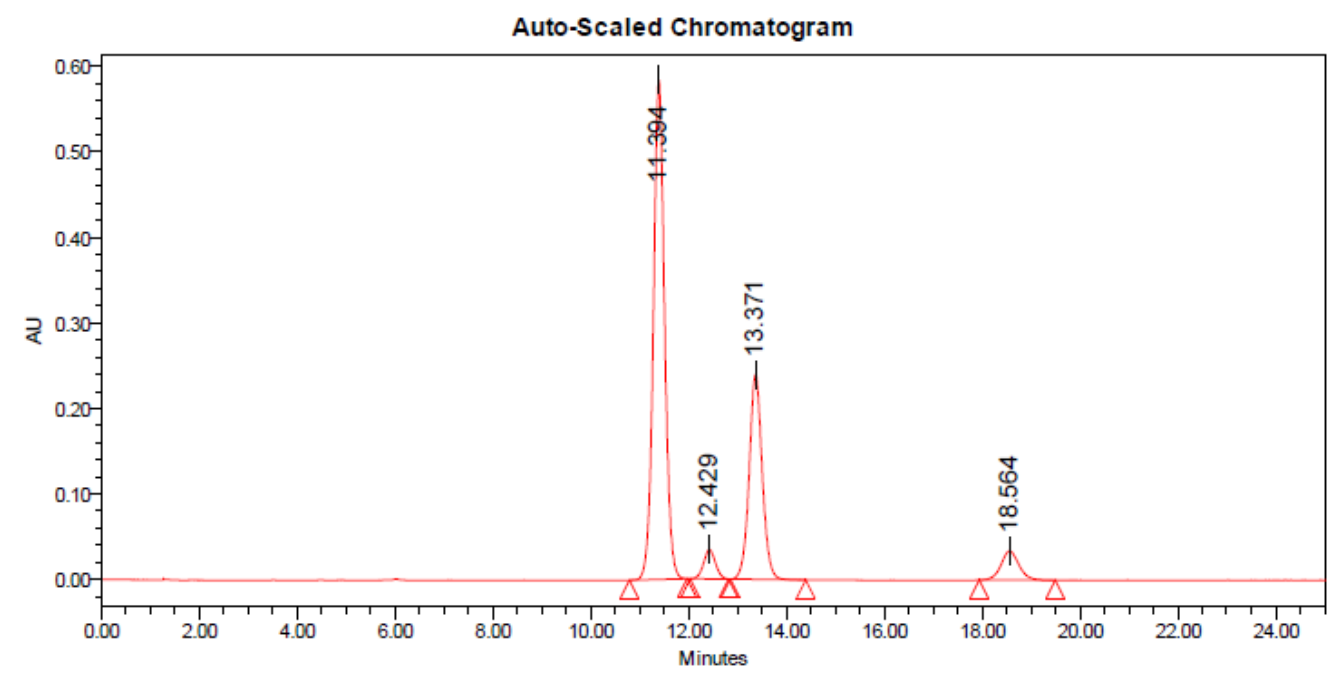

Processed Channel: PDA Ch1 254nm@1.2nm

-Compens.

\begin{tabular}{|l|l|r|r|r|r|}
\hline & Processed Channel & $\begin{array}{r}\text { Retention } \\
\text { Time }(\min )\end{array}$ & Area & $\%$ Area & Height \\
\hline 1 & PDA Ch1 254nm@1.2nm -Compens. & 11.394 & 9223521 & 61.10 & 583616 \\
\hline 2 & PDA Ch1 254nm@1.2nm -Compens. & 12.429 & 576266 & 3.82 & 34241 \\
\hline 3 & PDA Ch1 254nm@1.2nm -Compens. & 13.371 & 4430982 & 29.35 & 239161 \\
\hline 4 & PDA Ch1 254nm@1.2nm -Compens. & 18.564 & 864107 & 5.72 & 34003 \\
\hline
\end{tabular}


4h - Chiral SFC (Whelk column, flow rate $=2 \mathrm{~mL} / \mathrm{min}, \mathrm{T}=40^{\circ} \mathrm{C}, 10 \% \mathrm{IPA} / \mathrm{CO}_{2}, \mathrm{t}=50 \mathrm{~min}$ ): major diastereomer. major at $25.8 \mathrm{~min}$, minor at $28.5 \mathrm{~min}, \mathbf{8 7 \%}$ ee and minor diastereoisomer. major at $30.7 \mathrm{~min}$, minor at $45.1 \mathrm{~min}, 67 \%$ ee.

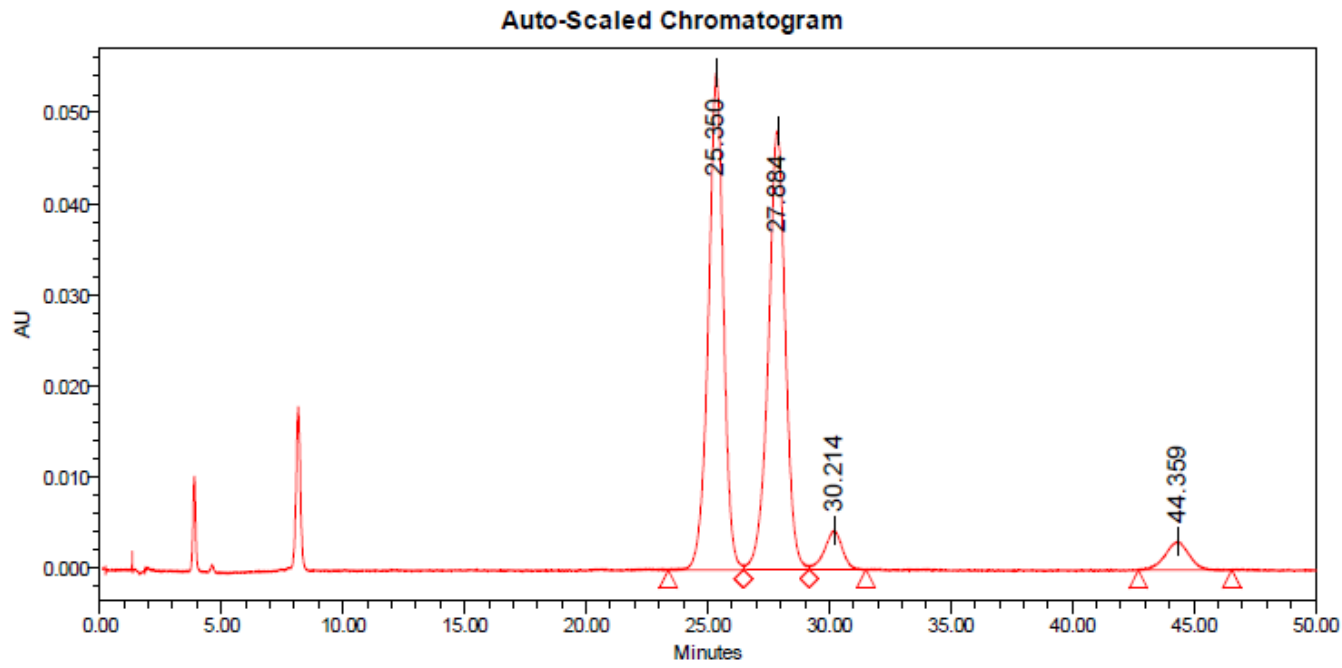

Processed Channel: PDA Ch1 254nm@1.2nm

-Compens.

\begin{tabular}{|l|l|r|r|r|r|}
\hline & \multicolumn{1}{|c|}{ Processed Channel } & $\begin{array}{r}\text { Retention } \\
\text { Time }(\mathrm{min})\end{array}$ & Area & $\%$ Area & Height \\
\hline 1 & PDA Ch1 254nm@1.2nm -Compens. & 25.350 & 2354220 & 45.82 & 54443 \\
\hline 2 & PDA Ch1 254nm@1.2nm -Compens. & 27.884 & 2359830 & 45.93 & 48127 \\
\hline 3 & PDA Ch1 254nm@1.2nm -Compens. & 30.214 & 214056 & 4.17 & 4268 \\
\hline 4 & PDA Ch1 254nm@1.2nm -Compens. & 44.359 & 209346 & 4.07 & 3155 \\
\hline
\end{tabular}

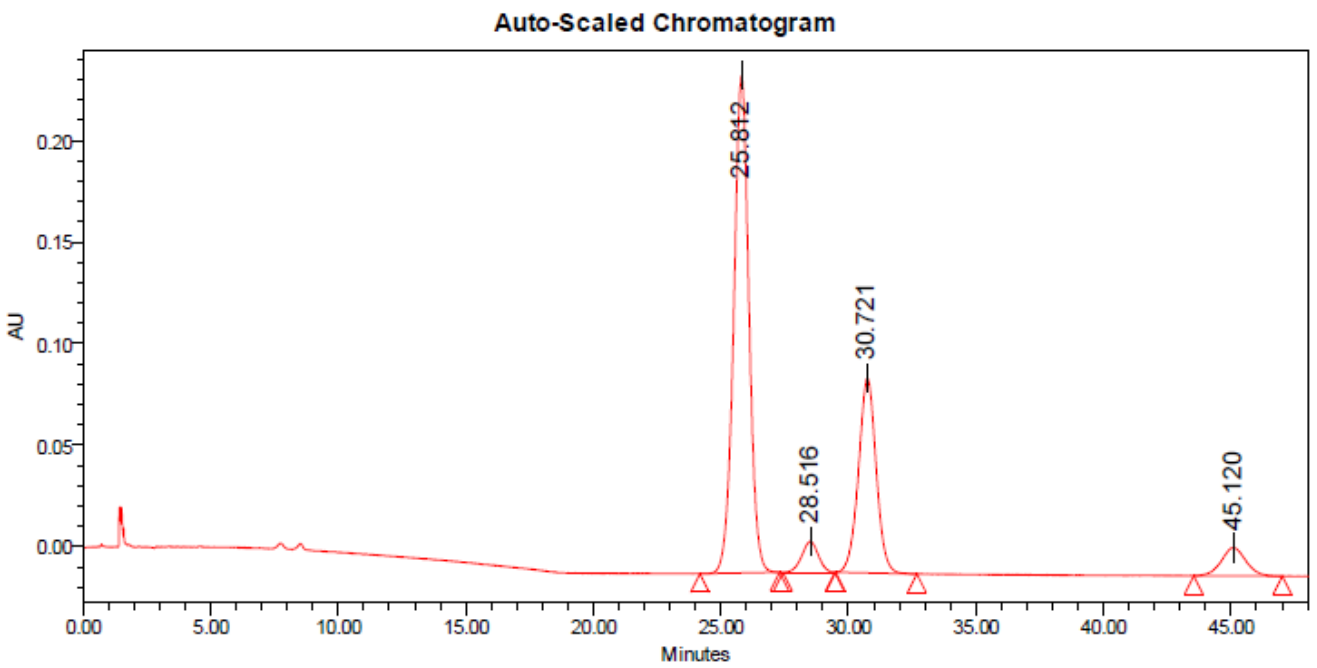

Processed Channel: PDA Ch1 254nm@1.2nm -Compens.

\begin{tabular}{|c|c|r|r|r|r|}
\hline & Processed Channel & $\begin{array}{r}\text { Retention } \\
\text { Time }(\min )\end{array}$ & Area & $\%$ Area & Height \\
\hline 1 & PDA Ch1 254nm@1.2nm -Compens. & 25.812 & 10116398 & 61.80 & 244746 \\
\hline 2 & PDA Ch1 254nm@1.2nm -Compens. & 28.516 & 690821 & 4.22 & 15363 \\
\hline 3 & PDA Ch1 254nm@1.2nm -Compens. & 30.721 & 4632272 & 28.30 & 95793 \\
\hline 4 & PDA Ch1 254nm@1.2nm -Compens. & 45.120 & 930237 & 5.68 & 13893 \\
\hline
\end{tabular}


$4 \mathbf{i}$ - Chiral SFC (AD3 column, flow rate $=2 \mathrm{~mL} / \mathrm{min}, \mathrm{T}=40{ }^{\circ} \mathrm{C}, 8 \%$ methanol $/ \mathrm{CO}_{2}, \mathrm{t}=15 \mathrm{~min}$ ): major diastereomer: major at $8.6 \mathrm{~min}$, minor at $6.4 \mathrm{~min}, 94 \%$ ee and minor diastereoisomer. major at $10.2 \mathrm{~min}$, minor at $7.5 \mathrm{~min}, 52 \%$ ee.

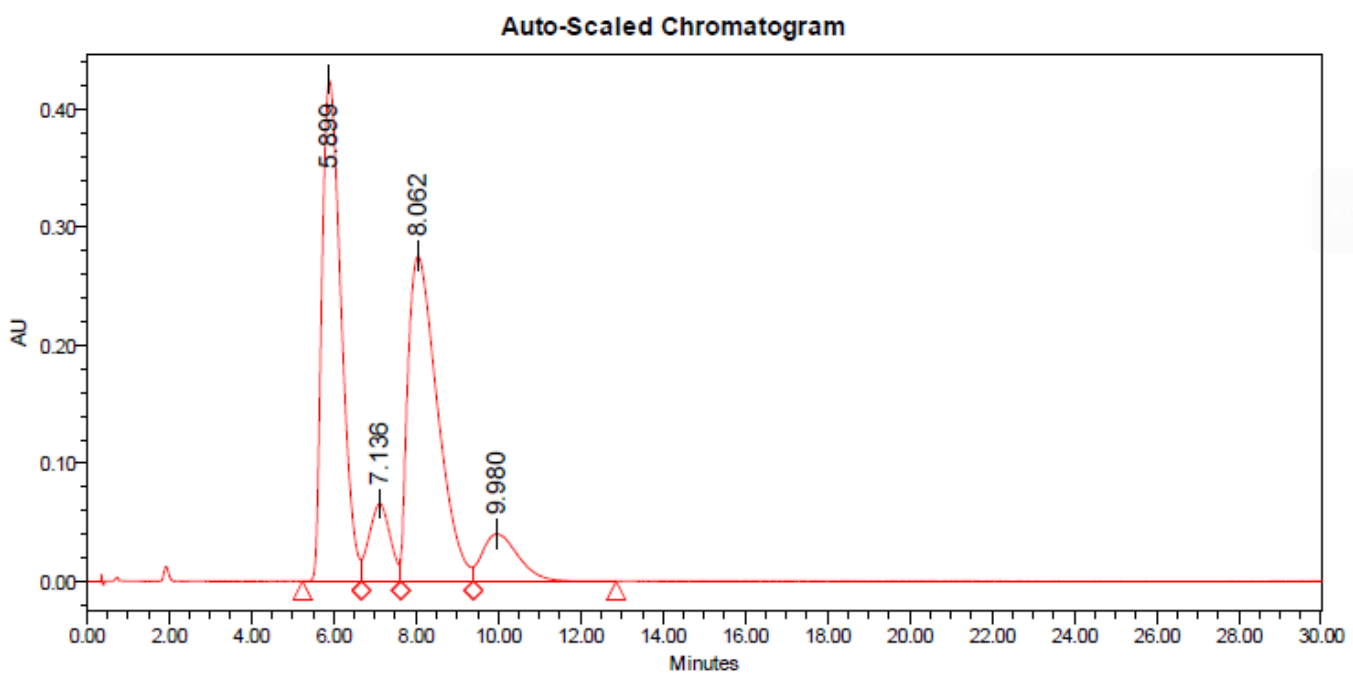

Processed Channel: PDA Ch1 254nm@1.2nm -Compens.

\begin{tabular}{|c|c|r|r|r|r|}
\hline & Processed Channel & $\begin{array}{r}\text { Retention } \\
\text { Time }(\mathbf{m i n})\end{array}$ & Area & $\%$ Area & Height \\
\hline 1 & PDA Ch1 254nm@1.2nm -Compens. & 5.899 & 13715661 & 42.63 & 424227 \\
\hline 2 & PDA Ch1 254nm@1.2nm -Compens. & 7.136 & 2326288 & 7.23 & 65705 \\
\hline 3 & PDA Ch1 254nm@1.2nm -Compens. & 8.062 & 13731184 & 42.68 & 275548 \\
\hline 4 & PDA Ch1 254nm@1.2nm -Compens. & 9.980 & 2400279 & 7.46 & 40042 \\
\hline
\end{tabular}

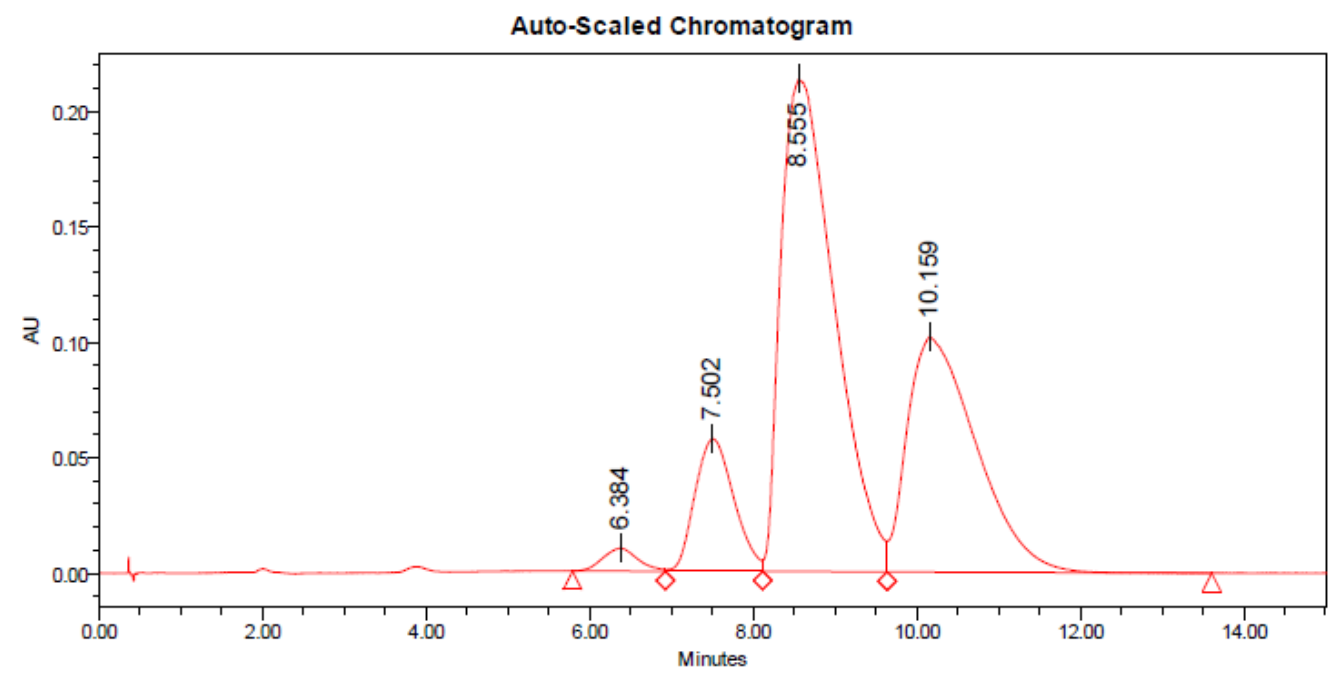

Processed Channel: PDA Ch1 254nm@1.2nm -Compens.

\begin{tabular}{|l|l|r|r|r|r|}
\hline & Processed Channel & $\begin{array}{r}\text { Retention } \\
\text { Time }(\min )\end{array}$ & Area & \% Area & Height \\
\hline 1 & PDA Ch1 254nm@1.2nm -Compens. & 6.384 & 288834 & 1.62 & 9834 \\
\hline 2 & PDA Ch1 254nm@1.2nm -Compens. & 7.502 & 1895041 & 10.61 & 57379 \\
\hline 3 & PDA Ch1 254nm@1.2nm-Compens. & 8.555 & 9716802 & 54.41 & 213078 \\
\hline 4 & PDA Ch1 254nm@1.2nm-Compens. & 10.159 & 5957949 & 33.36 & 102010 \\
\hline
\end{tabular}


4j - Chiral SFC (OJ3 column, flow rate $=2 \mathrm{~mL} / \mathrm{min}, \mathrm{T}=40{ }^{\circ} \mathrm{C}, 10 \%$ methanol $/ \mathrm{CO}_{2}, \mathrm{t}=20 \mathrm{~min}$ ): major diastereomer: major at $4.0 \mathrm{~min}$, minor at $8.2 \mathrm{~min}, 98 \%$ ee. Minor diastereoisomer could not be separated.

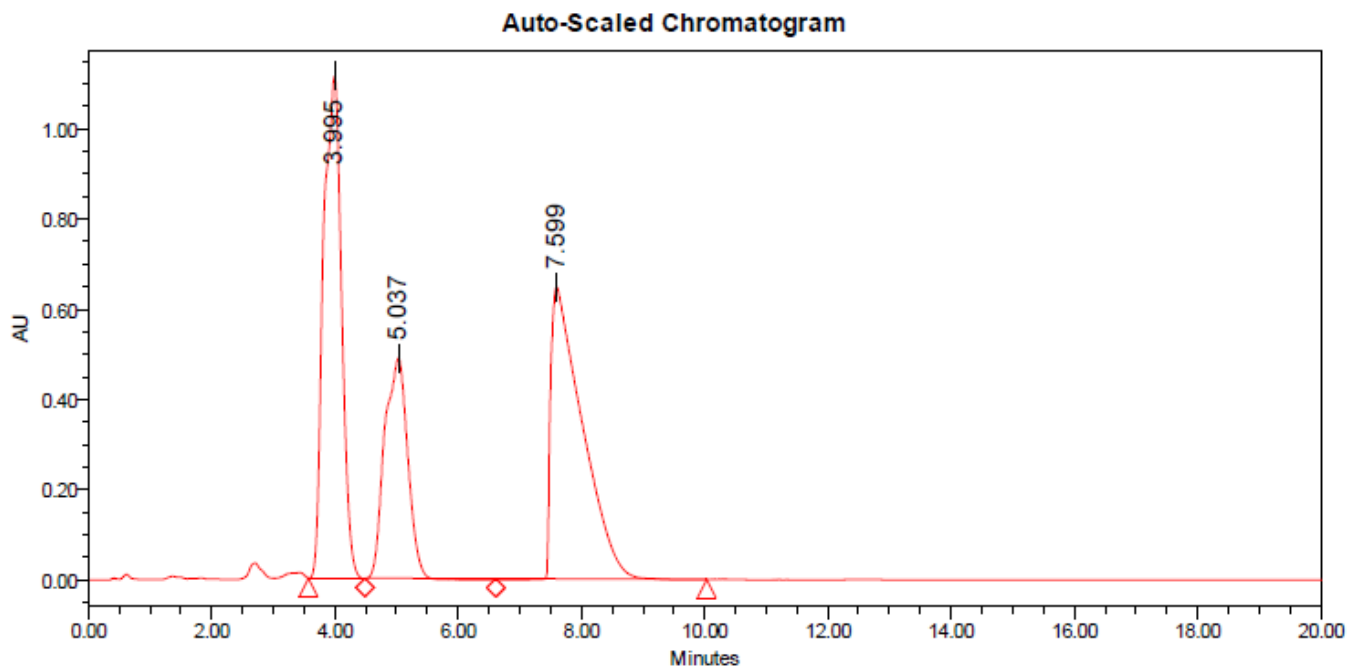

Processed Channel: PDA Ch1 254nm@1.2nm

-Compens.

\begin{tabular}{|c|c|r|r|r|r|}
\hline & Processed Channel & $\begin{array}{r}\text { Retention } \\
\text { Time (min) }\end{array}$ & Area & \% Area & Height \\
\hline 1 & PDA Ch1 254nm@1.2nm -Compens. & 3.995 & 22638486 & 38.73 & 1112012 \\
\hline 2 & PDA Ch1 254nm@1.2nm -Compens. & 5.037 & 12803442 & 21.90 & 488299 \\
\hline 3 & PDA Ch1 254nm@1.2nm -Compens. & 7.599 & 23017184 & 39.37 & 646830 \\
\hline
\end{tabular}

Auto-Scaled Chromatogram

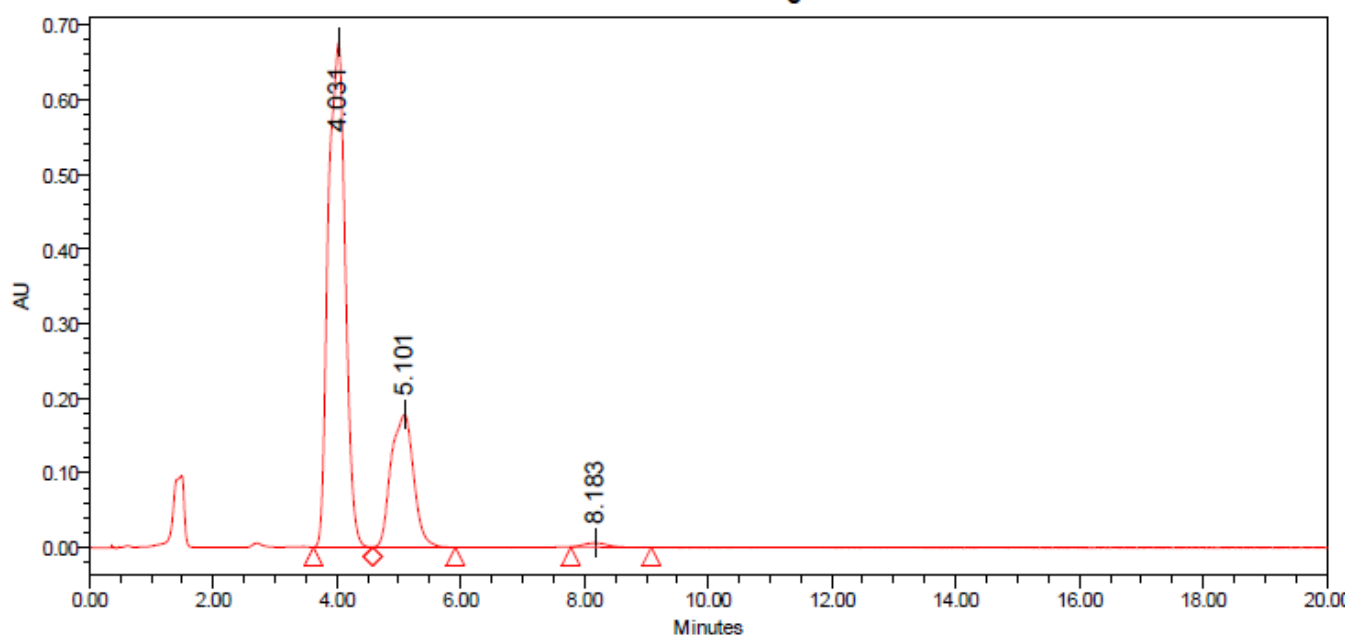

Processed Channel: PDA Ch1 254nm@1.2nm

-Compens.

\begin{tabular}{|l|l|r|r|r|r|}
\hline & Processed Channel & $\begin{array}{c}\text { Retention } \\
\text { Time }(\mathrm{min})\end{array}$ & Area & $\%$ Area & Height \\
\hline 1 & PDA Ch1 254nm@1.2nm -Compens. & 4.031 & 12925789 & 73.66 & 675684 \\
\hline 2 & PDA Ch1 254nm@1.2nm -Compens. & 5.101 & 4479572 & 25.53 & 177897 \\
\hline 3 & PDA Ch1 254nm@1.2nm -Compens. & 8.183 & 141995 & 0.81 & 5618 \\
\hline
\end{tabular}


4k - Chiral SFC (AD3 column, flow rate $=2 \mathrm{~mL} / \mathrm{min}, \mathrm{T}=40{ }^{\circ} \mathrm{C}, 15 \%$ methanol $/ \mathrm{CO}_{2}, \mathrm{t}=16 \mathrm{~min}$ ): major diastereomer: major at $7.0 \mathrm{~min}$, minor at $14.7 \mathrm{~min}, 99 \%$ ee.

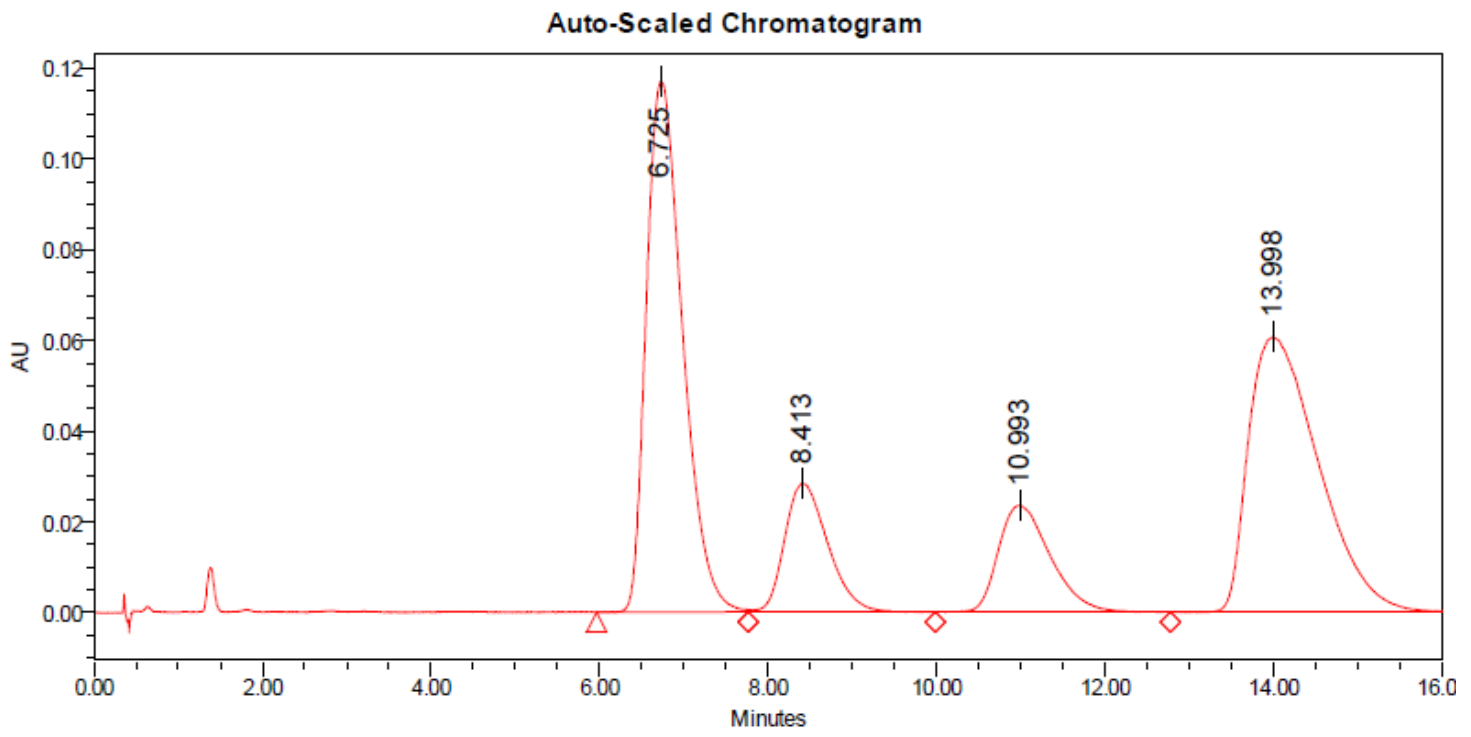

Processed Channel: PDA Ch1 254nm@1.2nm

-Compens.

\begin{tabular}{|c|c|r|r|r|r|}
\hline & Processed Channel & $\begin{array}{r}\text { Retention } \\
\text { Time (min) }\end{array}$ & Area & \% Area & Height \\
\hline 1 & PDA Ch1 254nm@1.2nm-Compens. & 6.725 & 3497188 & 38.89 & 117067 \\
\hline 2 & PDA Ch1 254nm@1.2nm-Compens. & 8.413 & 1006631 & 11.19 & 28350 \\
\hline 3 & PDA Ch1 254nm@1.2nm-Compens. & 10.993 & 997578 & 11.09 & 23489 \\
\hline 4 & PDA Ch1 254nm@1.2nm-Compens. & 13.998 & 3490779 & 38.82 & 60709 \\
\hline
\end{tabular}

Auto-Scaled Chromatogram

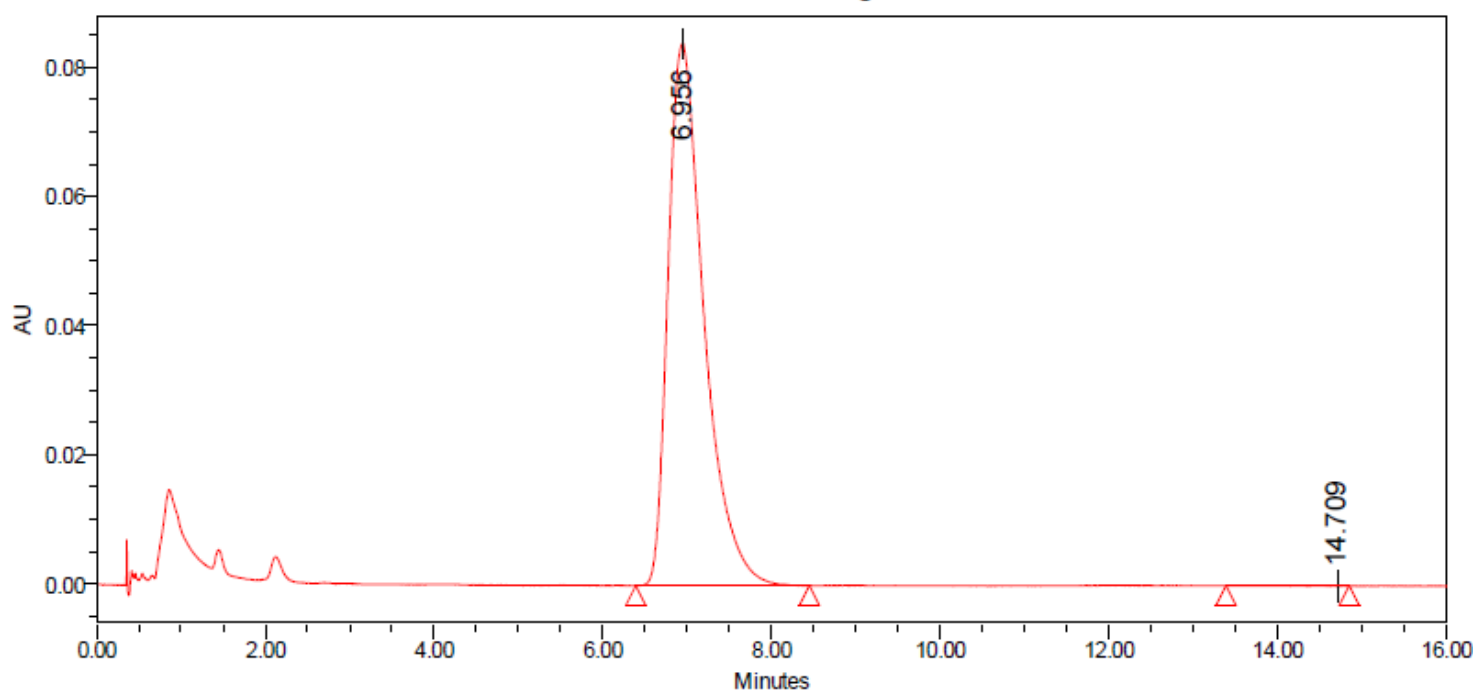

Processed Channel: PDA Ch1 254nm@1.2nm

-Compens.

\begin{tabular}{|c|c|r|r|r|r|}
\hline & Processed Channel & $\begin{array}{c}\text { Retention } \\
\text { Time (min) }\end{array}$ & Area & \% Area & Height \\
\hline 1 & PDA Ch1 254nm@1.2nm-Compens. & 6.956 & 2480338 & 99.94 & 83886 \\
\hline 2 & PDA Ch1 254nm@1.2nm-Compens. & 14.709 & 1500 & 0.06 & -84 \\
\hline
\end{tabular}


4I - Chiral SFC (Trefoil column, flow rate $=2 \mathrm{~mL} / \mathrm{min}, \mathrm{T}=40^{\circ} \mathrm{C}, 8 \% \mathrm{IPA} / \mathrm{CO}_{2}, \mathrm{t}=40 \mathrm{~min}$ ): major diastereomer. major at $20.3 \mathrm{~min}$, minor at $16.8 \mathrm{~min}, 96 \%$ ee.

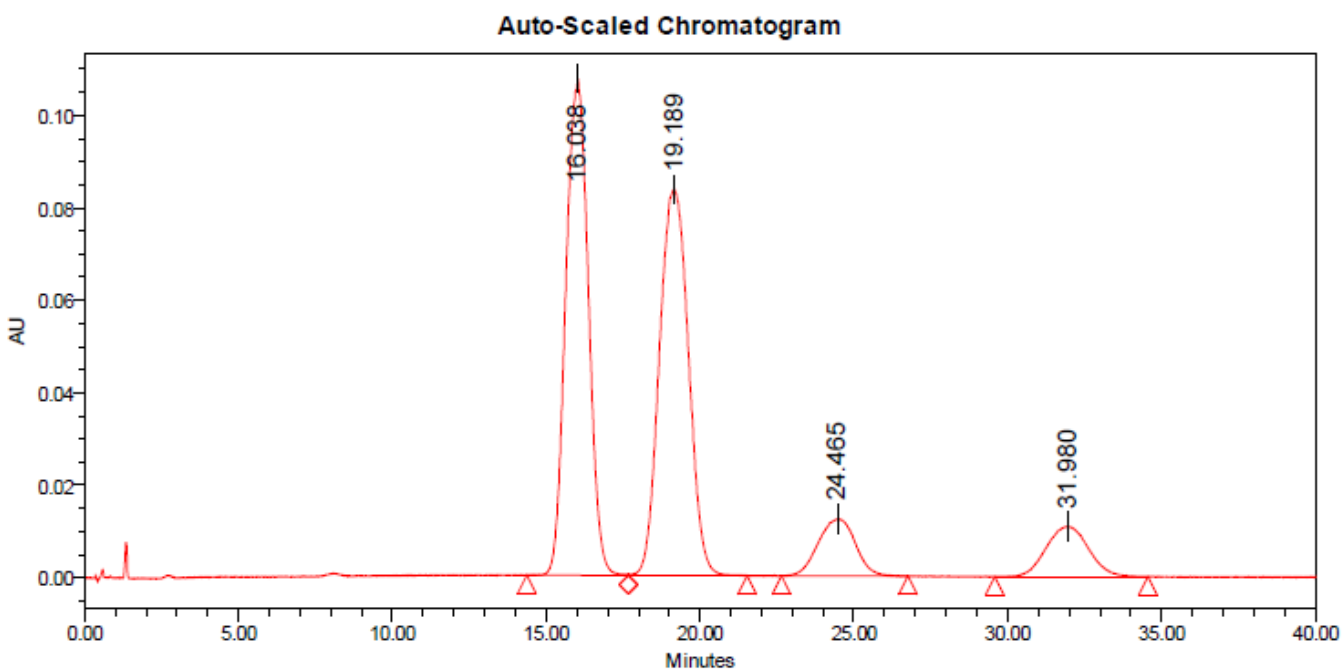

Processed Channel: PDA Ch1 254nm@1.2nm -Compens.

\begin{tabular}{|l|l|r|r|r|r|}
\hline & Processed Channel & $\begin{array}{r}\text { Retention } \\
\text { Time (min) }\end{array}$ & Area & $\%$ Area & Height \\
\hline 1 & PDA Ch1 254nm@1.2nm -Compens. & 16.038 & 5462503 & 42.04 & 107252 \\
\hline 2 & PDA Ch1 254nm@1.2nm -Compens. & 19.189 & 5472853 & 42.12 & 83440 \\
\hline 3 & PDA Ch1 254nm@1.2nm -Compens. & 24.465 & 1021296 & 7.86 & 12309 \\
\hline 4 & PDA Ch1 254nm@1.2nm -Compens. & 31.980 & 1036024 & 7.97 & 10891 \\
\hline
\end{tabular}

Auto-Scaled Chromatogram

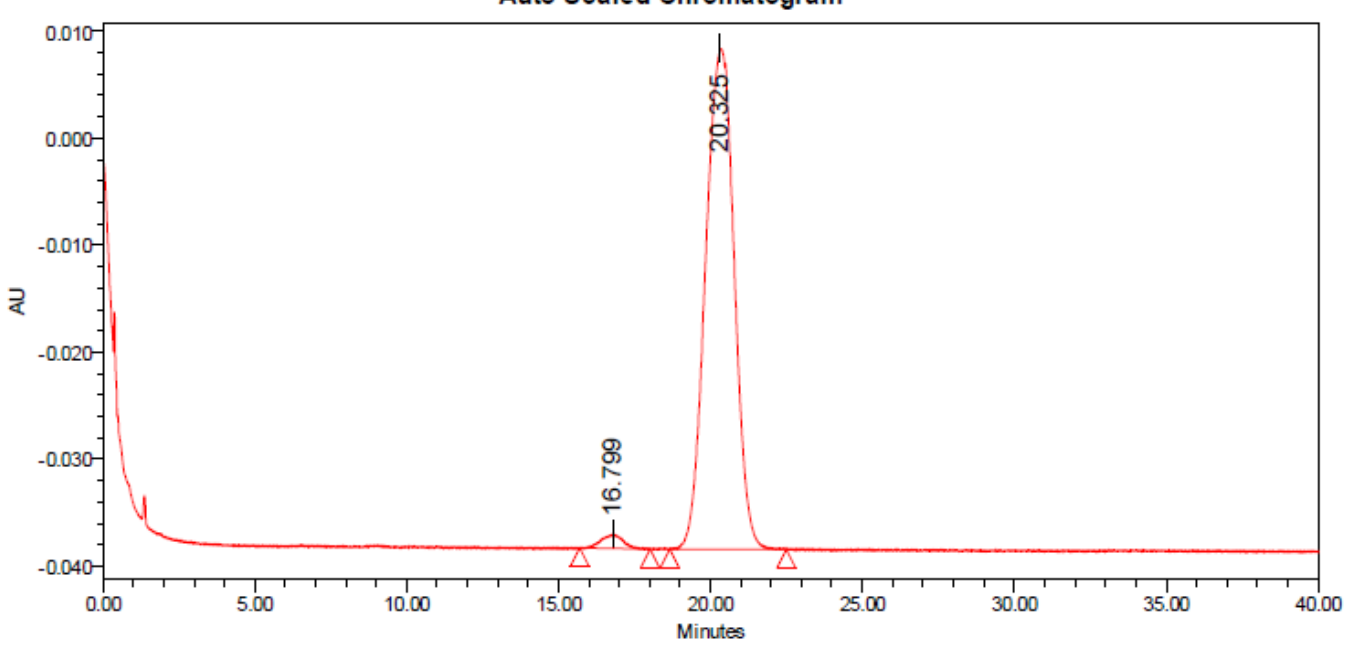

Processed Channel: PDA Ch1 254nm@1.2nm -Compens.

\begin{tabular}{|l|c|r|r|r|r|}
\hline & Processed Channel & $\begin{array}{r}\text { Retention } \\
\text { Time }(\boldsymbol{m i n})\end{array}$ & Area & \% Area & Height \\
\hline 1 & PDA Ch1 254nm@1.2nm -Compens. & 16.799 & 64415 & 2.06 & 1358 \\
\hline 2 & PDA Ch1 254nm@1.2nm -Compens. & 20.325 & 3066195 & 97.94 & 46808 \\
\hline
\end{tabular}


$4 \mathrm{~m}$ - Chiral SFC (Trefoil, flow rate $=2 \mathrm{~mL} / \mathrm{min}, \mathrm{T}=40{ }^{\circ} \mathrm{C}, 8 \% \quad \mathrm{IPA} / \mathrm{CO}_{2}, \mathrm{t}=25 \mathrm{~min}$ ): major diastereomer. major at $11.8 \mathrm{~min}$, minor at $12.8 \mathrm{~min}, 99 \%$ ee and minor diastereoisomer. major at $20.0 \mathrm{~min}$, minor at $18.0 \mathrm{~min}, 10 \%$ ee.

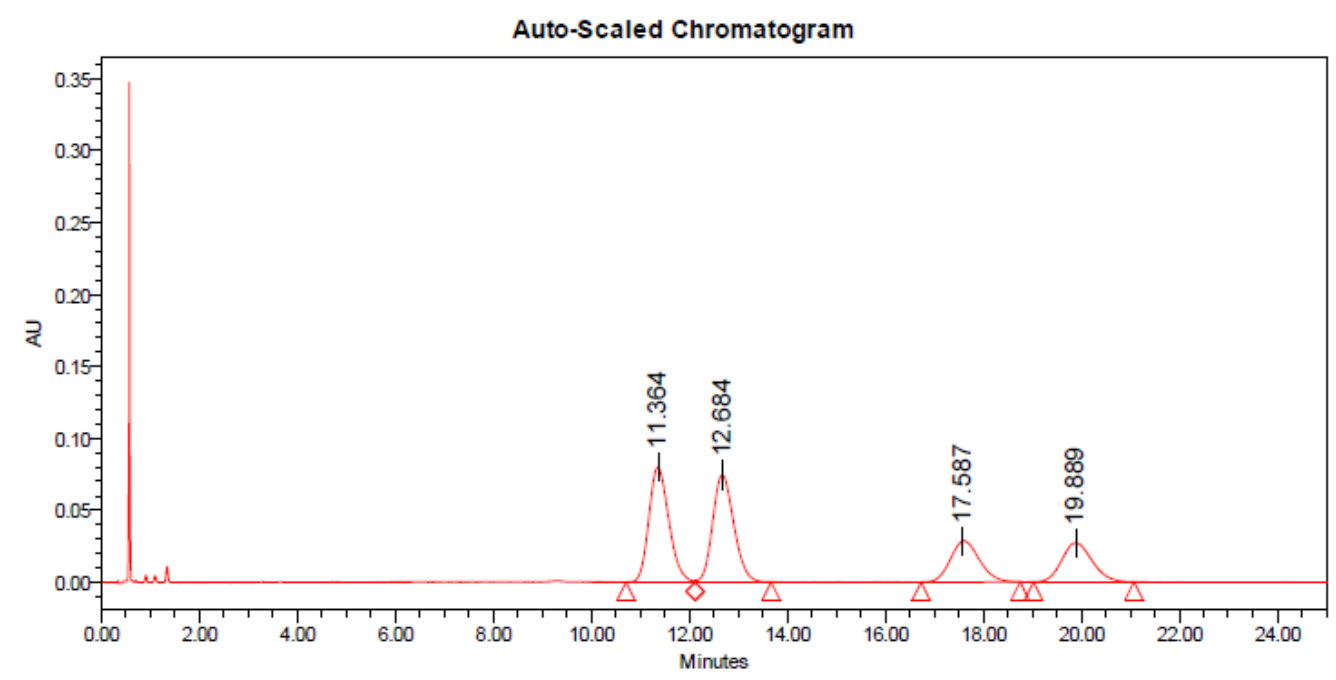

Processed Channel: PDA Ch1 254nm@1.2nm

-Compens.

\begin{tabular}{|l|l|r|r|r|r|}
\hline & Processed Channel & $\begin{array}{c}\text { Retention } \\
\text { Time }(\mathrm{min})\end{array}$ & Area & $\%$ Area & Height \\
\hline 1 & PDA Ch1 254nm@1.2nm -Compens. & 11.364 & 2263359 & 33.01 & 79898 \\
\hline 2 & PDA Ch1 254nm@1.2nm -Compens. & 12684 & 2241310 & 32.69 & 74547 \\
\hline 3 & PDA Ch1 254nm@1.2nm -Compens. & 17.587 & 1179053 & 17.20 & 28844 \\
\hline 4 & PDA Ch1 254nm@1.2nm -Compens. & 19.889 & 1172253 & 17.10 & 27217 \\
\hline
\end{tabular}

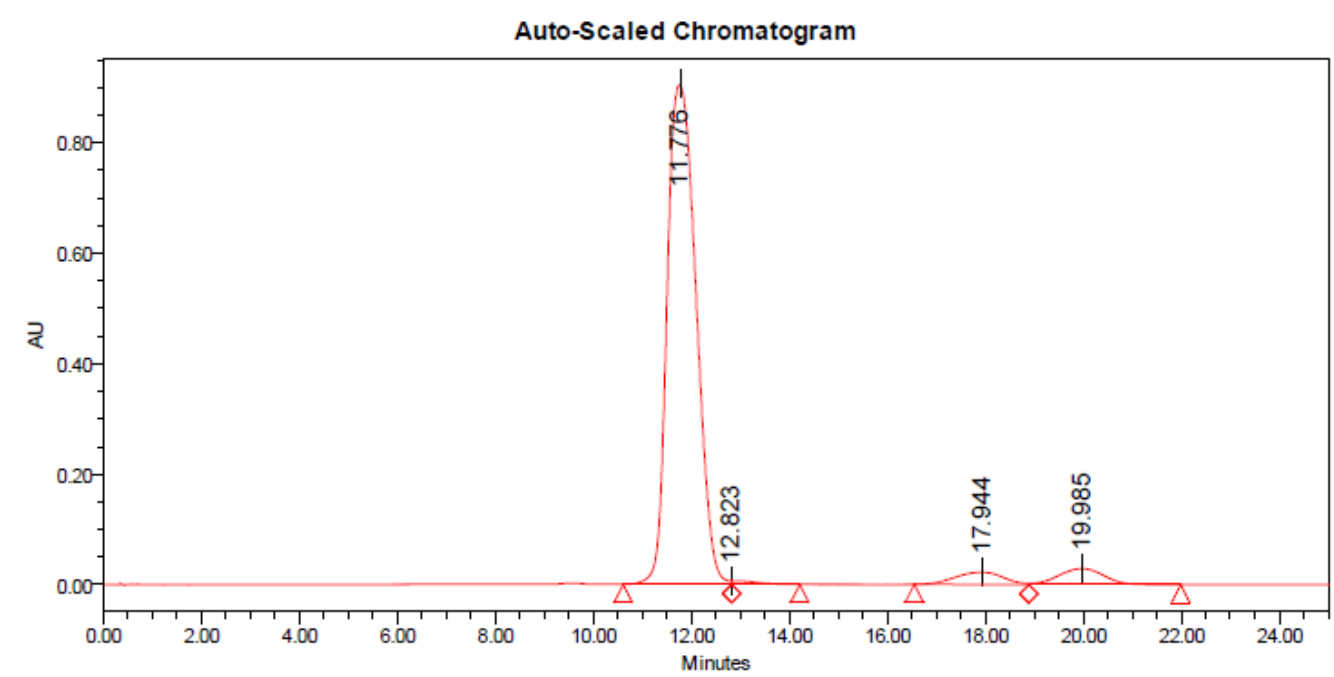

Processed Channel: PDA Ch1 254nm@1.2nm

-Compens.

\begin{tabular}{|c|c|r|r|r|r|}
\hline & Processed Channel & $\begin{array}{r}\text { Retention } \\
\text { Time ( } \min )\end{array}$ & Area & \% Area & Height \\
\hline 1 & PDA Ch1 254nm@1.2nm -Compens. & 11.776 & 36915168 & 91.21 & 905021 \\
\hline 2 & PDA Ch1 254nm@1.2nm -Compens. & 12823 & 226998 & 0.56 & 6406 \\
\hline 3 & PDA Ch1 254nm@1.2nm -Compens. & 17.944 & 1497582 & 3.70 & 22135 \\
\hline 4 & PDA Ch1 254nm@1.2nm -Compens. & 19.985 & 1831735 & 4.53 & 28886 \\
\hline
\end{tabular}


4n - Chiral HPLC (ODH column, flow rate $=0.5 \mathrm{~mL} / \mathrm{min}, \mathrm{T}=25^{\circ} \mathrm{C}, 20 \% \mathrm{IPA} / n$-hexane, $\mathrm{t}=50$ min): major diastereomer: major at $30.2 \mathrm{~min}$, minor at $27.0 \mathrm{~min}, 96 \%$ ee and minor diastereoisomer. major at $35.6 \mathrm{~min}$, minor at $21.4 \mathrm{~min}, 18 \%$ ee.
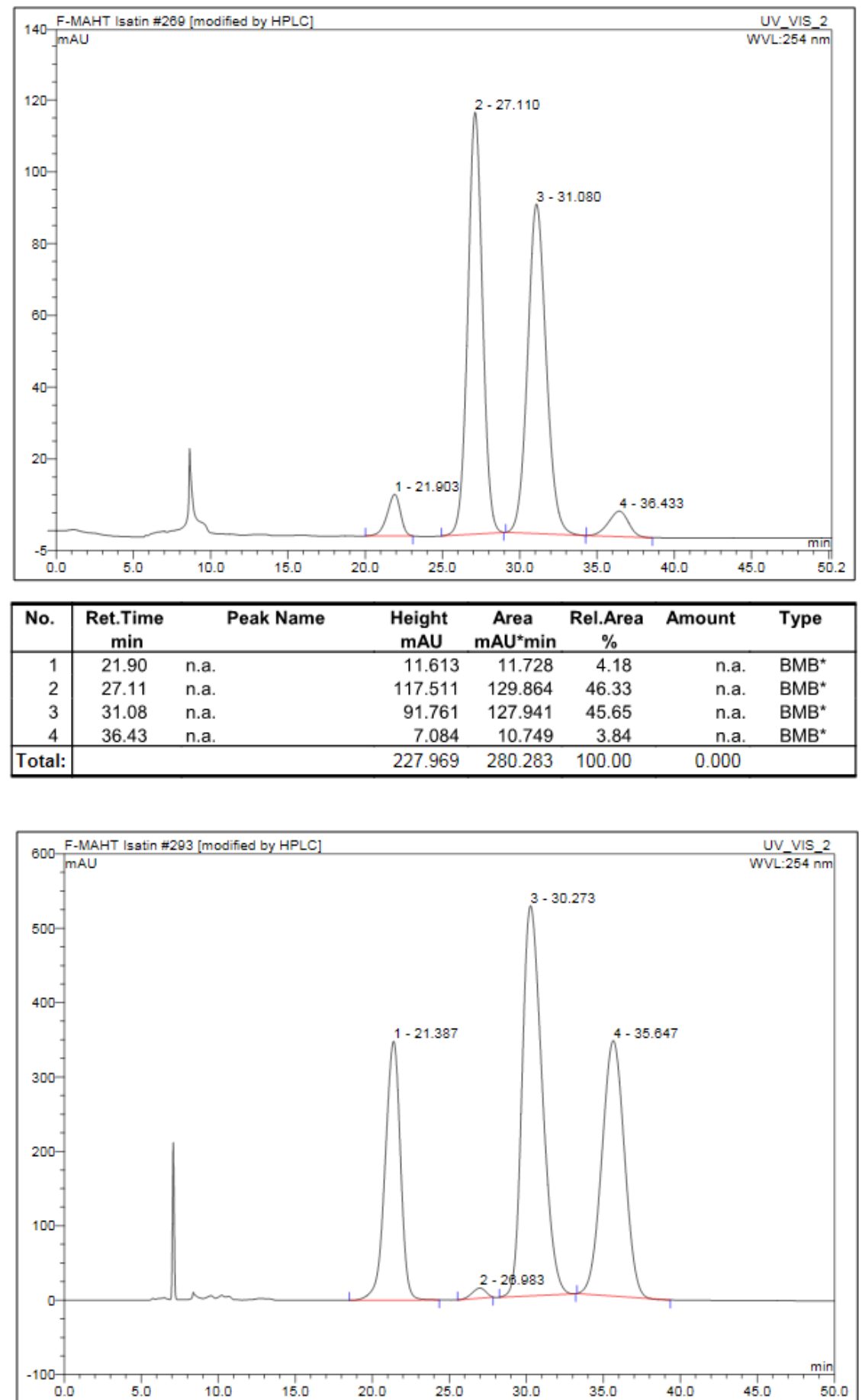

\begin{tabular}{|r|ccrrrrr|}
\hline No. & $\begin{array}{c}\text { Ret.Time } \\
\text { min }\end{array}$ & Peak Name & $\begin{array}{c}\text { Height } \\
\text { mAU }\end{array}$ & $\begin{array}{c}\text { Area } \\
\text { mAU*min }\end{array}$ & $\begin{array}{r}\text { Rel.Area } \\
\%\end{array}$ & Amount & Type \\
\hline 1 & 21.39 & n.a. & 348.054 & 401.398 & 22.53 & n.a. & BMB $^{\star}$ \\
2 & 26.98 & n.a. & 13.540 & 14.010 & 0.79 & n.a. & BMB $^{\star}$ \\
3 & 30.27 & n.a. & 524.383 & 785.243 & 44.08 & n.a. & BMB $^{\star}$ \\
4 & 35.65 & n.a. & 343.335 & 580.801 & 32.60 & n.a. & BMB $^{*}$ \\
\hline Total: & & & 1229.312 & 1781.453 & 100.00 & 0.000 & \\
\hline
\end{tabular}


40 - Chiral SFC (Trefoil column, flow rate $=2 \mathrm{~mL} / \mathrm{min}, \mathrm{T}=40{ }^{\circ} \mathrm{C}, 20 \%$ methanol $/ \mathrm{CO}_{2}, \mathrm{t}=15 \mathrm{~min}$ ): major diastereomer: major at $2.5 \mathrm{~min}$, minor at $4.6 \mathrm{~min}, 92 \%$ ee and minor diastereoisomer. major at $8.1 \mathrm{~min}$, minor at $3.3 \mathrm{~min}, 7 \%$ ee.

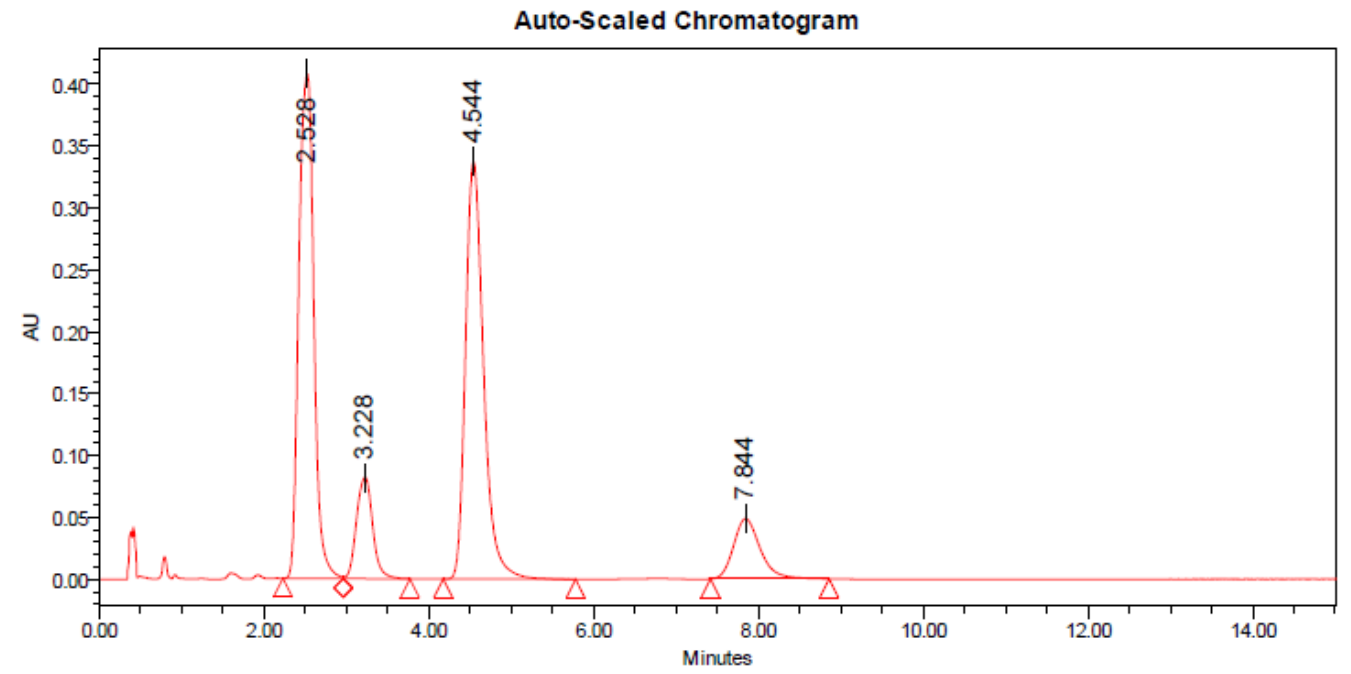

Processed Channel: PDA Ch1 254nm@1.2nm -Compens.

\begin{tabular}{|c|c|r|r|r|r|}
\hline & Processed Channel & $\begin{array}{r}\text { Retention } \\
\text { Time (min) }\end{array}$ & Area & \% Area & Height \\
\hline 1 & PDA Ch1 254nm@1.2nm -Compens. & 2.528 & 5114565 & 41.06 & 407354 \\
\hline 2 & PDA Ch1 254nm@1.2nm -Compens. & 3.228 & 111792 & 8.98 & 81766 \\
\hline 3 & PDA Ch1 254nm@1.2nm -Compens. & 4.544 & 5128124 & 41.17 & 337167 \\
\hline 4 & PDA Ch1 254nm@1.2nm -Compens. & 7.844 & 1095167 & 8.79 & 48748 \\
\hline
\end{tabular}

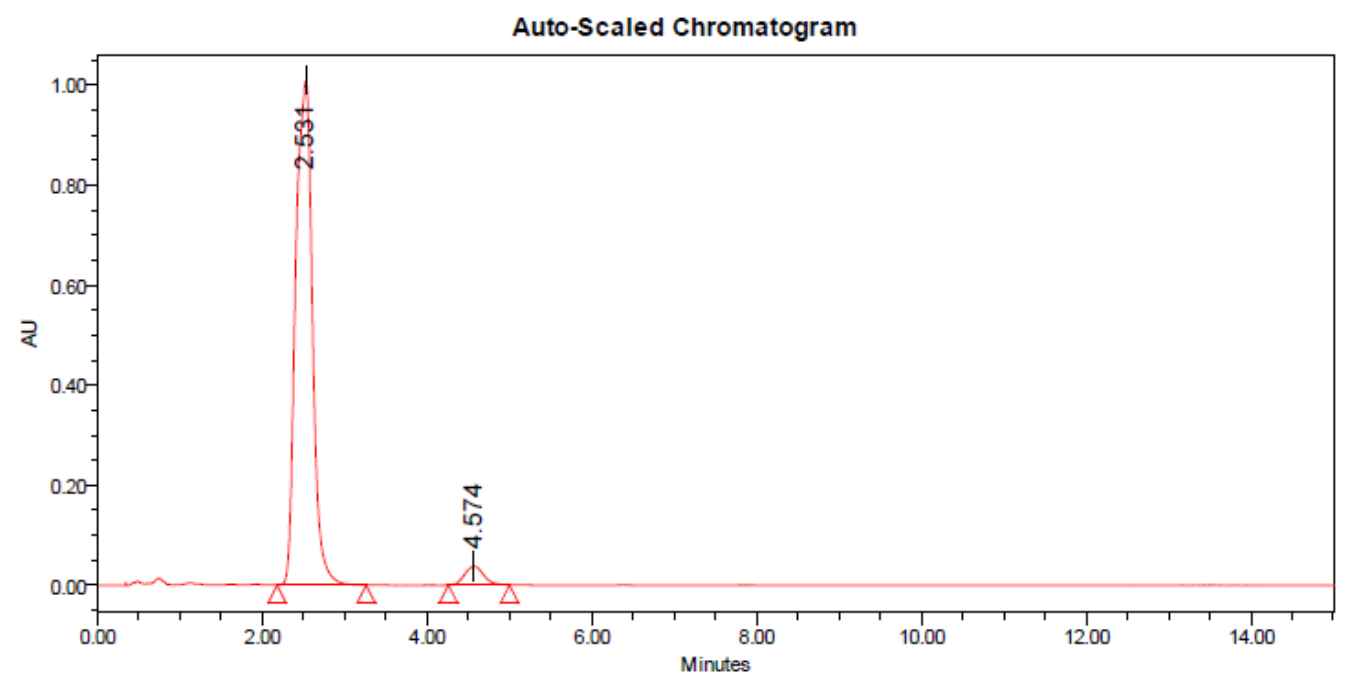

Processed Channel: PDA Ch1 254nm@1.2nm -Compens.

\begin{tabular}{|l|c|r|r|r|r|}
\hline & Processed Channel & $\begin{array}{r}\text { Retention } \\
\text { Time }(\min )\end{array}$ & Area & \% Area & Height \\
\hline 1 & PDA Ch1 254nm@1.2nm -Compens. & 2.531 & 14121800 & 95.96 & 1008584 \\
\hline 2 & PDA Ch1 254nm@1.2nm -Compens. & 4.574 & 593789 & 4.04 & 37460 \\
\hline
\end{tabular}




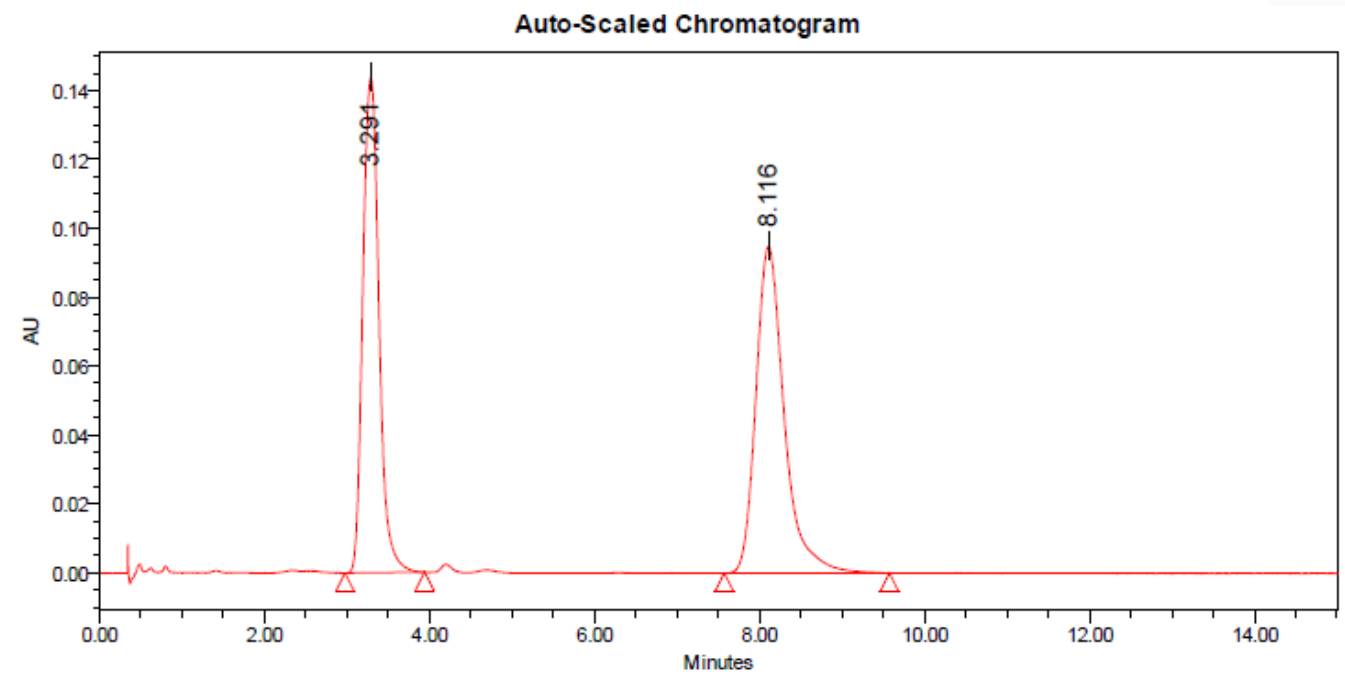

Processed Channel: PDA Ch1 254nm@1.2nm

$$
\text { -Compens. }
$$

\begin{tabular}{|l|l|r|r|r|r|}
\hline & \multicolumn{1}{|c|}{ Processed Channel } & $\begin{array}{c}\text { Retention } \\
\text { Time }(\min )\end{array}$ & Area & $\%$ Area & Height \\
\hline 1 & PDA Ch1 254nm@1.2nm-Compens. & 3.291 & 1954957 & 46.48 & 143675 \\
\hline 2 & PDA Ch1 254nm@1.2nm-Compens. & 8.116 & 2251501 & 53.52 & 94914 \\
\hline
\end{tabular}


$4 p$ - Chiral SFC (OJ3 column, flow rate $=2 \mathrm{~mL} / \mathrm{min}, \mathrm{T}=40{ }^{\circ} \mathrm{C}, 10 \%$ methanol $/ \mathrm{CO}_{2}, \mathrm{t}=25 \mathrm{~min}$ ): major diastereomer: major at $18.2 \mathrm{~min}$, minor at $14.4 \mathrm{~min}, 93 \%$ ee and minor diastereoisomer: major at $16.8 \mathrm{~min}$, minor at $13.5 \mathrm{~min}, 61 \%$ ee.

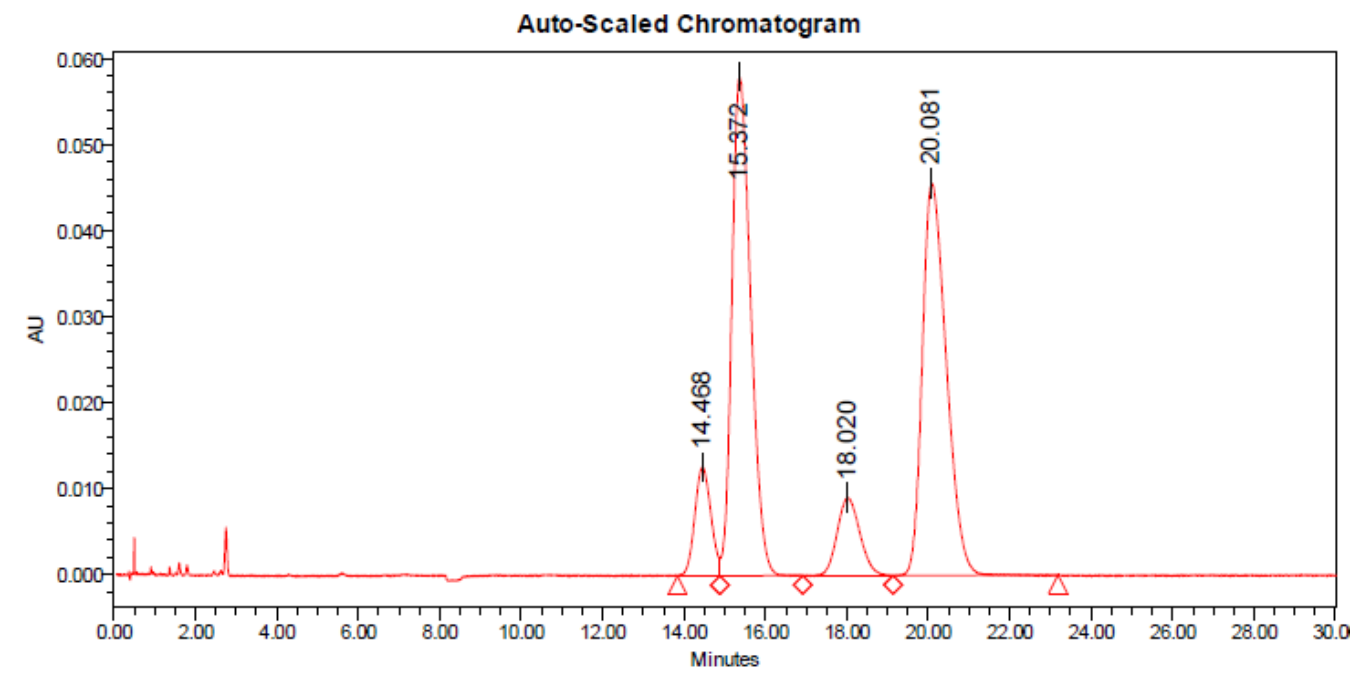

Processed Channel: PDA Ch1 254nm@1.2nm

-Compens.

\begin{tabular}{|c|c|r|r|r|r|}
\hline & Processed Channel & $\begin{array}{r}\text { Retention } \\
\text { Time }(\min )\end{array}$ & Area & \% Area & Height \\
\hline 1 & PDA Ch1 254nm@1.2nm -Compens. & 14.468 & 347162 & 7.83 & 12676 \\
\hline 2 & PDA Ch1 254nm@1.2nm -Compens. & 15.372 & 1868721 & 42.17 & 57968 \\
\hline 3 & PDA Ch1 254nm@1.2nm-Compens. & 18.020 & 349527 & 7.89 & 9052 \\
\hline 4 & PDA Ch1 254nm@1.2nm-Compens. & 20.081 & 1865513 & 42.10 & 45624 \\
\hline
\end{tabular}

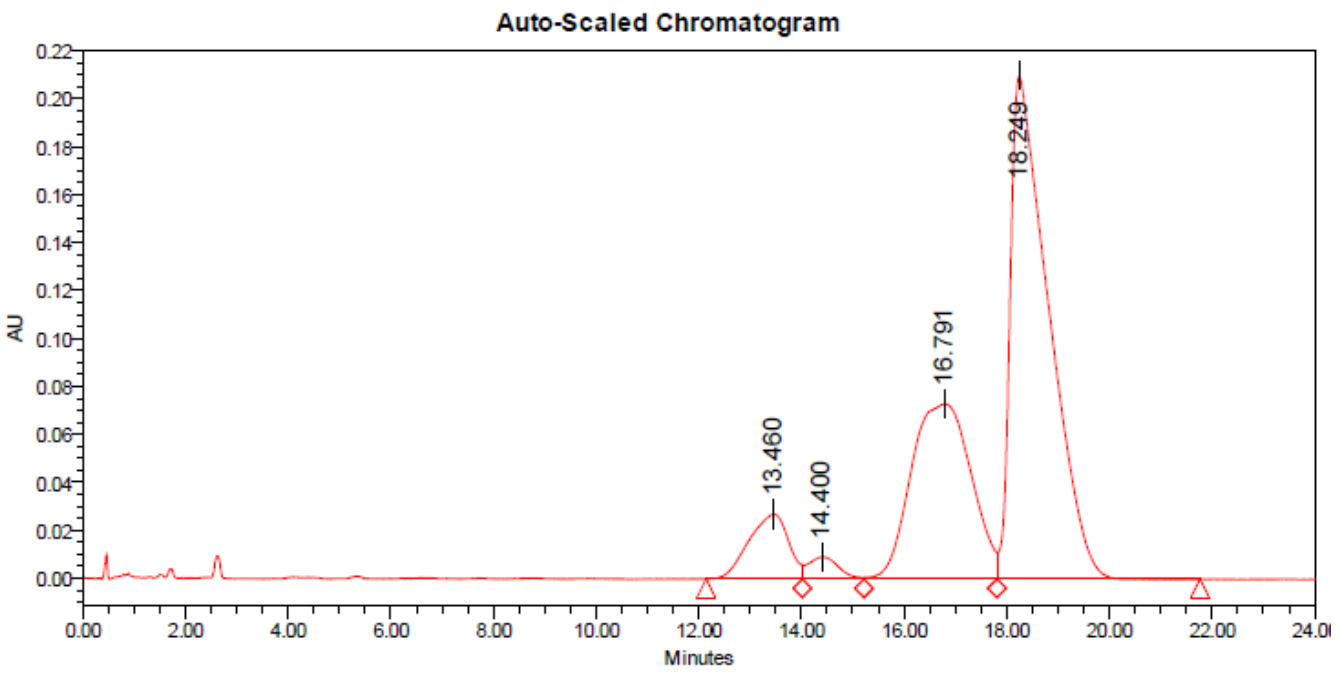

Processed Channel: PDA Ch1 254nm@1.2nm

-Compens.

\begin{tabular}{|l|l|r|r|r|r|}
\hline & Processed Channel & $\begin{array}{r}\text { Retention } \\
\text { Time ( } \min )\end{array}$ & Area & \% Area & Height \\
\hline 1 & PDA Ch1 254nm@1.2nm -Compens. & 13.460 & 1446382 & 7.76 & 27058 \\
\hline 2 & PDA Ch1 254nm@1.2nm -Compens. & 14.400 & 378437 & 2.03 & 9262 \\
\hline 3 & PDA Ch1 254nm@1.2nm -Compens. & 16.791 & 5929261 & 31.81 & 73214 \\
\hline 4 & PDA Ch1 254nm@1.2nm -Compens. & 18.249 & 10885505 & 58.40 & 209777 \\
\hline
\end{tabular}


4q - Chiral SFC (Whelk column, flow rate $=2 \mathrm{~mL} / \mathrm{min}, \mathrm{T}=40^{\circ} \mathrm{C}, 10 \% \mathrm{IPA} / \mathrm{CO}_{2}, \mathrm{t}=20 \mathrm{~min}$ ): major diastereomer. major at $10.6 \mathrm{~min}$, minor at $13.4 \mathrm{~min}, 83 \%$ ee and minor diastereoisomer. major at $11.7 \mathrm{~min}$, minor at $16.4 \mathrm{~min}, 58 \%$ ee.

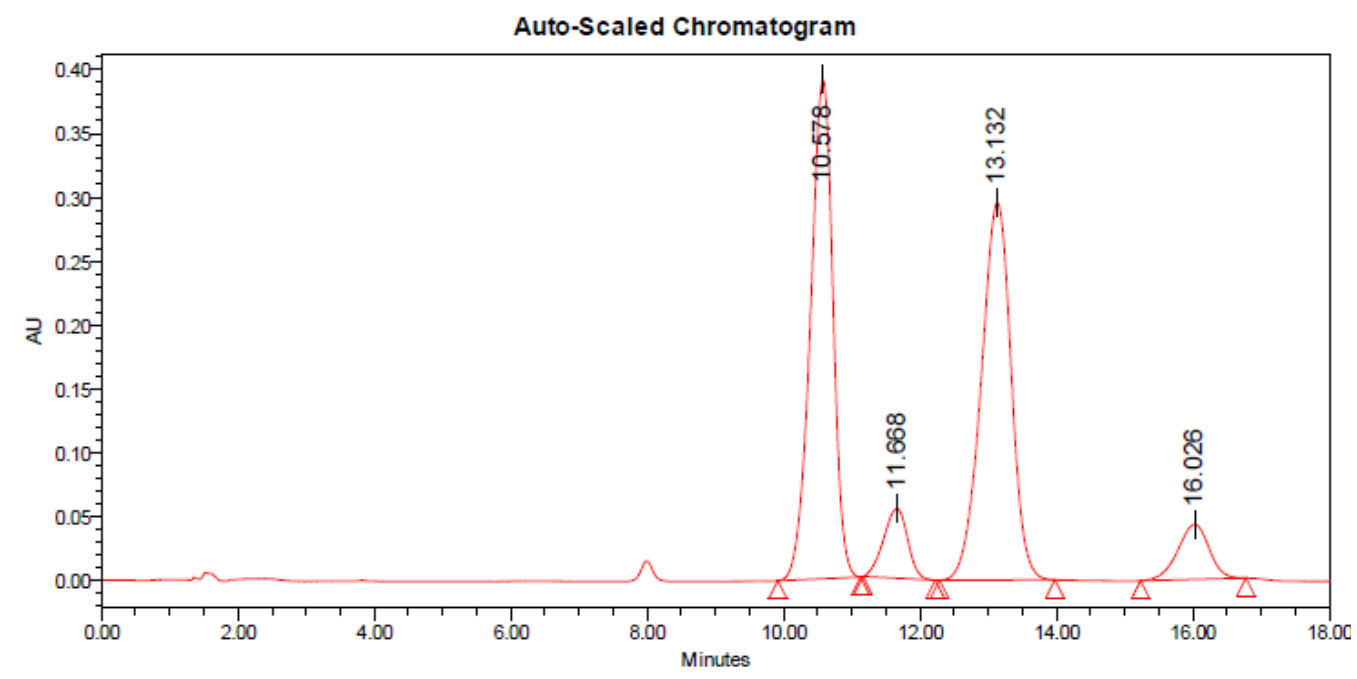

Processed Channel: PDA Ch1 254nm@1.2nm

-Compens.

\begin{tabular}{|l|l|r|r|r|r|}
\hline & Processed Channel & $\begin{array}{r}\text { Retention } \\
\text { Time ( }(\mathbf{m i n})\end{array}$ & Area & $\%$ Area & Height \\
\hline 1 & PDA Ch1 254nm@1.2nm -Compens. & 10.578 & 8957425 & 43.30 & 390573 \\
\hline 2 & PDA Ch1 254nm@1.2nm -Compens. & 11.668 & 1336487 & 6.46 & 54706 \\
\hline 3 & PDA Ch1 254nm@1.2nm -Compens. & 13.132 & 9019826 & 43.60 & 295370 \\
\hline 4 & PDA Ch1 254nm@1.2nm -Compens. & 16.026 & 1374570 & 6.64 & 43195 \\
\hline
\end{tabular}

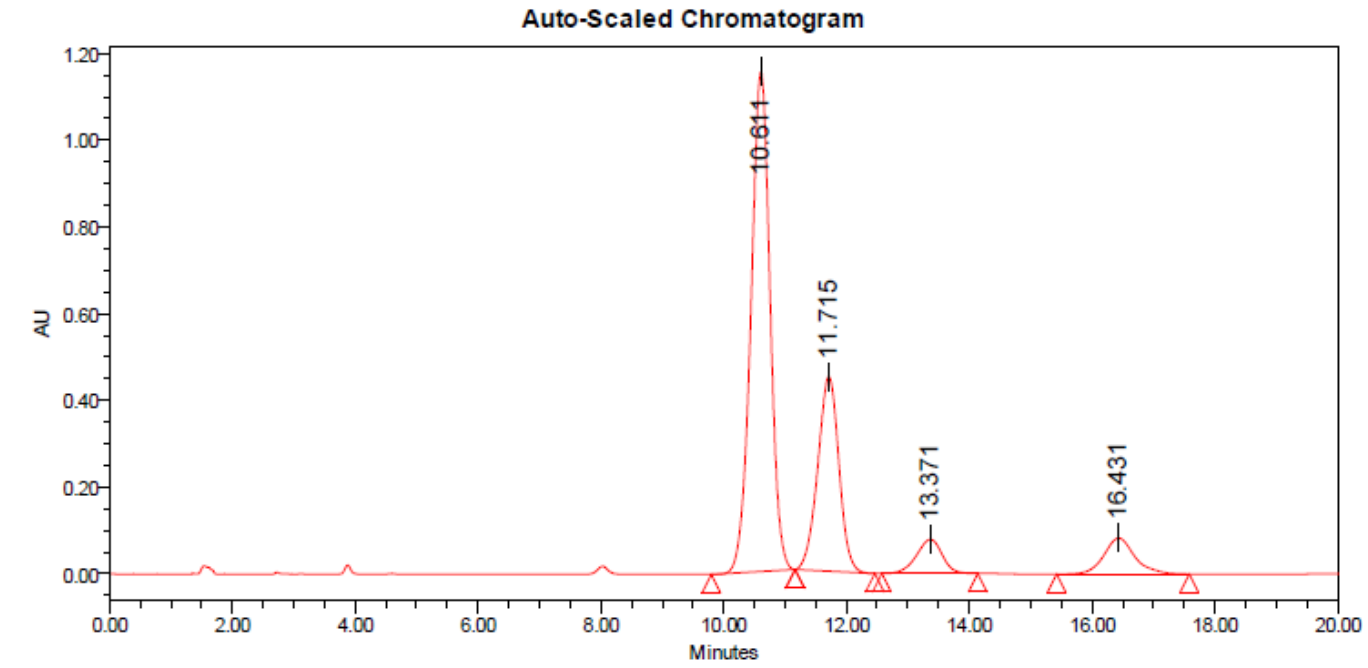

Processed Channel: PDA Ch1 254nm@1.2nm

-Compens.

\begin{tabular}{|l|l|r|r|r|r|}
\hline & Processed Channel & $\begin{array}{c}\text { Retention } \\
\text { Time (min) }\end{array}$ & Area & $\%$ Area & Height \\
\hline 1 & PDA Ch1 254nm@1.2nm-Compens. & 10.611 & 24860394 & 60.88 & 1150910 \\
\hline 2 & PDA Ch1 254nm@1.2nm-Compens. & 11.715 & 10779574 & 26.40 & 448252 \\
\hline 3 & PDA Ch1 254nm@1.2nm -Compens. & 13.371 & 2300717 & 5.63 & 78391 \\
\hline 4 & PDA Ch1 254nm@1.2nm-Compens. & 16.431 & 2896582 & 7.09 & 83414 \\
\hline
\end{tabular}


4r - Chiral SFC (AD3 column, flow rate $=2 \mathrm{~mL} / \mathrm{min}, \mathrm{T}=40{ }^{\circ} \mathrm{C}, 15 \%$ methanol $/ \mathrm{CO}_{2}, \mathrm{t}=20 \mathrm{~min}$ ): major diastereomer: major at $2.5 \mathrm{~min}$, minor at $2.0 \mathrm{~min}, 87 \%$ ee and minor diastereoisomer. major at $5.0 \mathrm{~min}$, minor at $3.2 \mathrm{~min}, 64 \%$ eee.

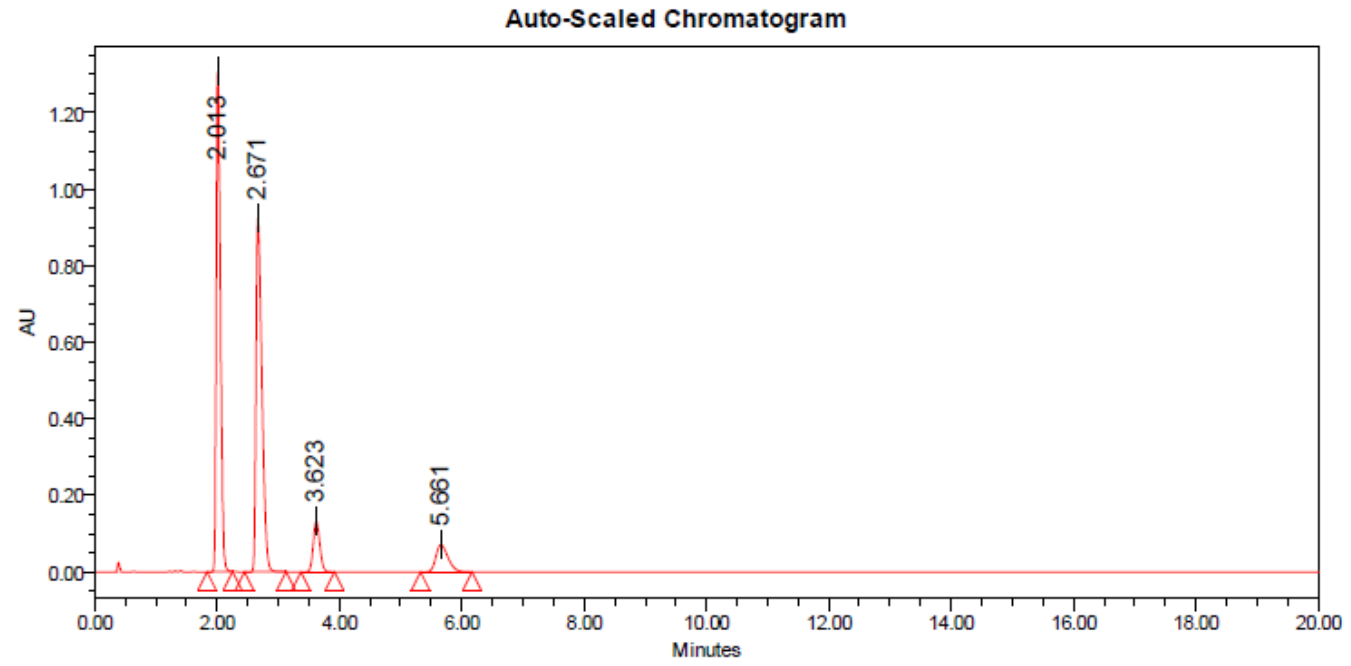

Processed Channel: PDA Ch1 254nm@1.2nm

-Compens.

\begin{tabular}{|l|l|r|r|r|r|}
\hline & Processed Channel & $\begin{array}{r}\text { Retention } \\
\text { Time (min) }\end{array}$ & Area & $\%$ Area & Height \\
\hline 1 & PDA Ch1 254nm@1.2nm -Compens. & 2.013 & 6225560 & 43.14 & 1305963 \\
\hline 2 & PDA Ch1 254nm@1.2nm -Compens. & 2.671 & 6241281 & 43.25 & 923564 \\
\hline 3 & PDA Ch1 254nm@1.2nm-Compens. & 3.623 & 987358 & 6.84 & 132126 \\
\hline 4 & PDA Ch1 254nm@1.2nm -Compens. & 5.661 & 975844 & 6.76 & 71058 \\
\hline
\end{tabular}

Auto-Scaled Chromatogram

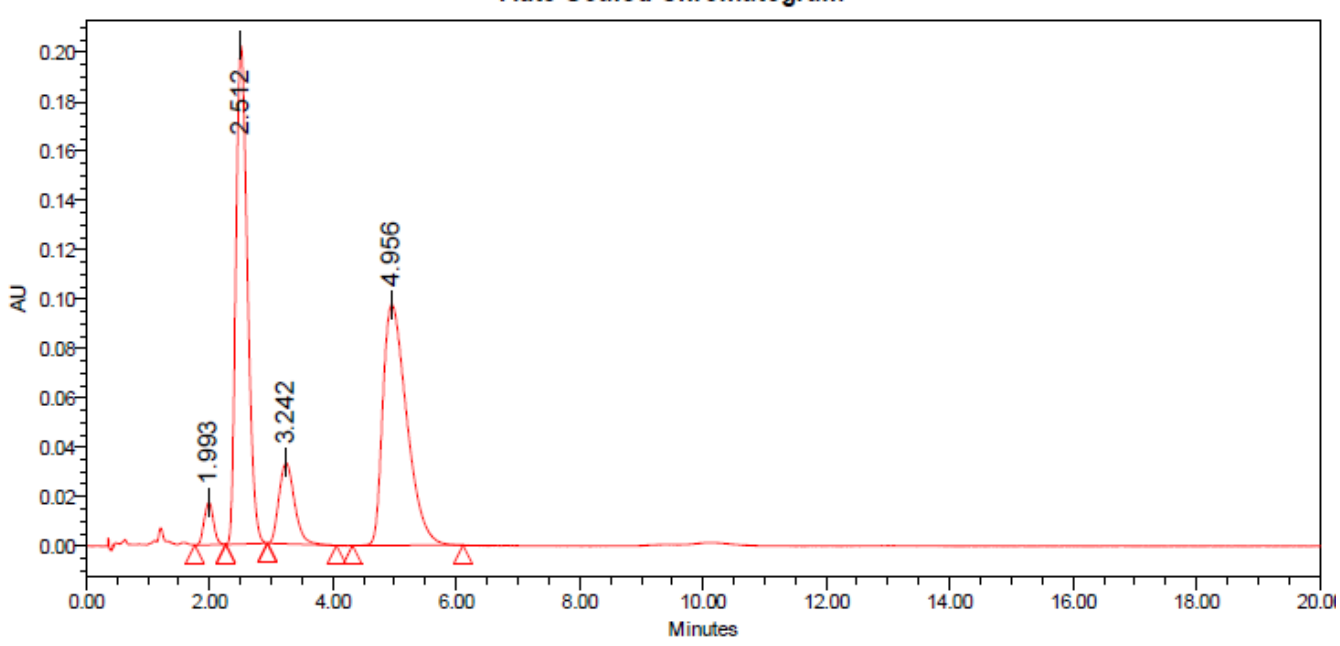

Processed Channel: PDA Ch1 254nm@1.2nm

-Compens.

\begin{tabular}{|c|c|r|r|r|r|}
\hline & Processed Channel & $\begin{array}{c}\text { Retention } \\
\text { Time (min) }\end{array}$ & Area & \% Area & Height \\
\hline 1 & PDA Ch1 254nm@1.2nm -Compens. & 1.993 & 182575 & 3.04 & 17250 \\
\hline 2 & PDA Ch1 254nm@1.2nm -Compens. & 2.512 & 2627237 & 43.71 & 201754 \\
\hline 3 & PDA Ch1 254nm@1.2nm -Compens. & 3.242 & 574700 & 9.56 & 32742 \\
\hline 4 & PDA Ch1 254nm@1.2nm -Compens. & 4.956 & 2625995 & 43.69 & 97532 \\
\hline
\end{tabular}


4s - Chiral SFC (Whelk column, flow rate $=2 \mathrm{~mL} / \mathrm{min}, \mathrm{T}=40^{\circ} \mathrm{C}, 5 \%$ methanol $/ \mathrm{CO}_{2}, \mathrm{t}=35 \mathrm{~min}$ ): major diastereomer: major at $17.7 \mathrm{~min}$, minor at $16.2 \mathrm{~min}, 84 \%$ ee and minor diastereoisomer: major at $19.2 \mathrm{~min}$, minor at $27.5 \mathrm{~min}, 58 \%$ ee.

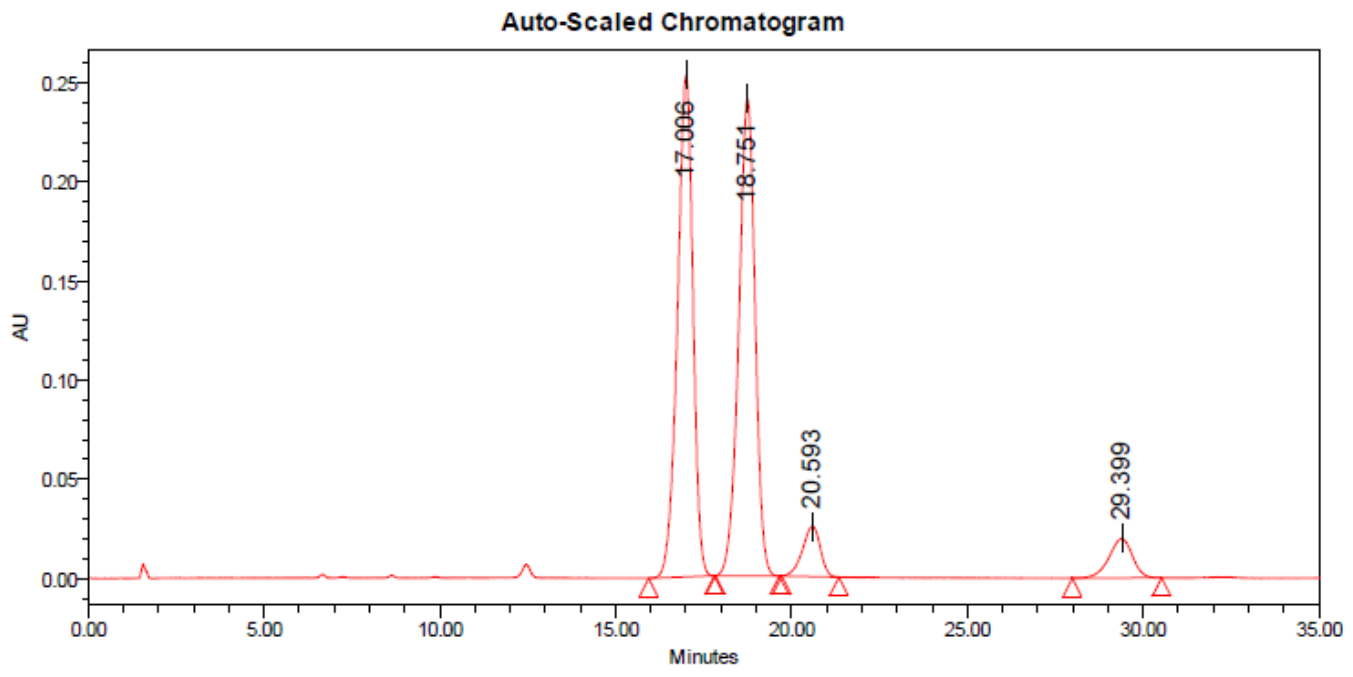

Processed Channel: PDA Ch1 254nm@1.2nm -Compens.

\begin{tabular}{|l|l|r|r|r|r|}
\hline & Processed Channel & $\begin{array}{c}\text { Retention } \\
\text { Time (min) }\end{array}$ & Area & $\%$ Area & Height \\
\hline 1 & PDA Ch1 254nm@1.2nm -Compens. & 17.006 & 7757057 & 44.76 & 252841 \\
\hline 2 & PDA Ch1 254nm@1.2nm -Compens. & 18.751 & 7761591 & 44.79 & 240603 \\
\hline 3 & PDA Ch1 254nm@1.2nm -Compens. & 20.593 & 894348 & 5.16 & 25292 \\
\hline 4 & PDA Ch1 254nm@1.2nm -Compens. & 29.399 & 916068 & 5.29 & 19827 \\
\hline
\end{tabular}

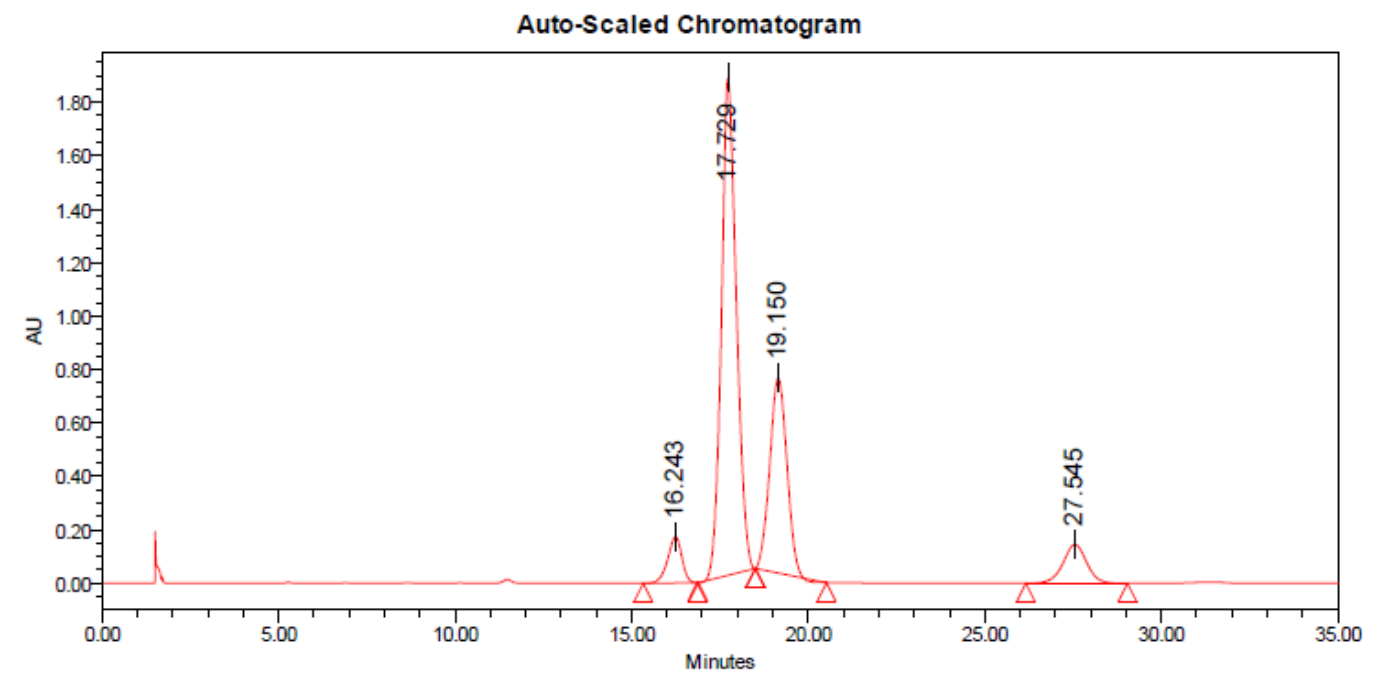

Processed Channel: PDA Ch1 254nm@1.2nm -Compens.

\begin{tabular}{|r|l|r|r|r|r|}
\hline & Processed Channel & $\begin{array}{r}\text { Retention } \\
\text { Time (min) }\end{array}$ & Area & $\%$ Area & Height \\
\hline 1 & PDA Ch1 254nm@1.2nm -Compens. & 16.243 & 4810157 & 5.27 & 173541 \\
\hline 2 & PDA Ch1 254nm@1.2nm -Compens. & 17.729 & 54983571 & 60.21 & 1861408 \\
\hline 3 & PDA Ch1 254nm@1.2nm -Compens. & 19.150 & 24853819 & 27.22 & 729200 \\
\hline 4 & PDA Ch1 254nm@1.2nm -Compens. & 27.545 & 6664969 & 7.30 & 145165 \\
\hline
\end{tabular}


4t - Chiral SFC (OJ3 column, flow rate $=2 \mathrm{~mL} / \mathrm{min}, \mathrm{T}=40{ }^{\circ} \mathrm{C}, 5 \% \mathrm{IPA} / \mathrm{CO}_{2}, \mathrm{t}=20 \mathrm{~min}$ ): major diastereomer. major at $4.4 \mathrm{~min}$, minor at $6.2 \mathrm{~min}, 88 \%$ ee and minor diastereoisomer: major at $16.0 \mathrm{~min}$, minor at $13.7 \mathrm{~min}, 88 \%$ ee.

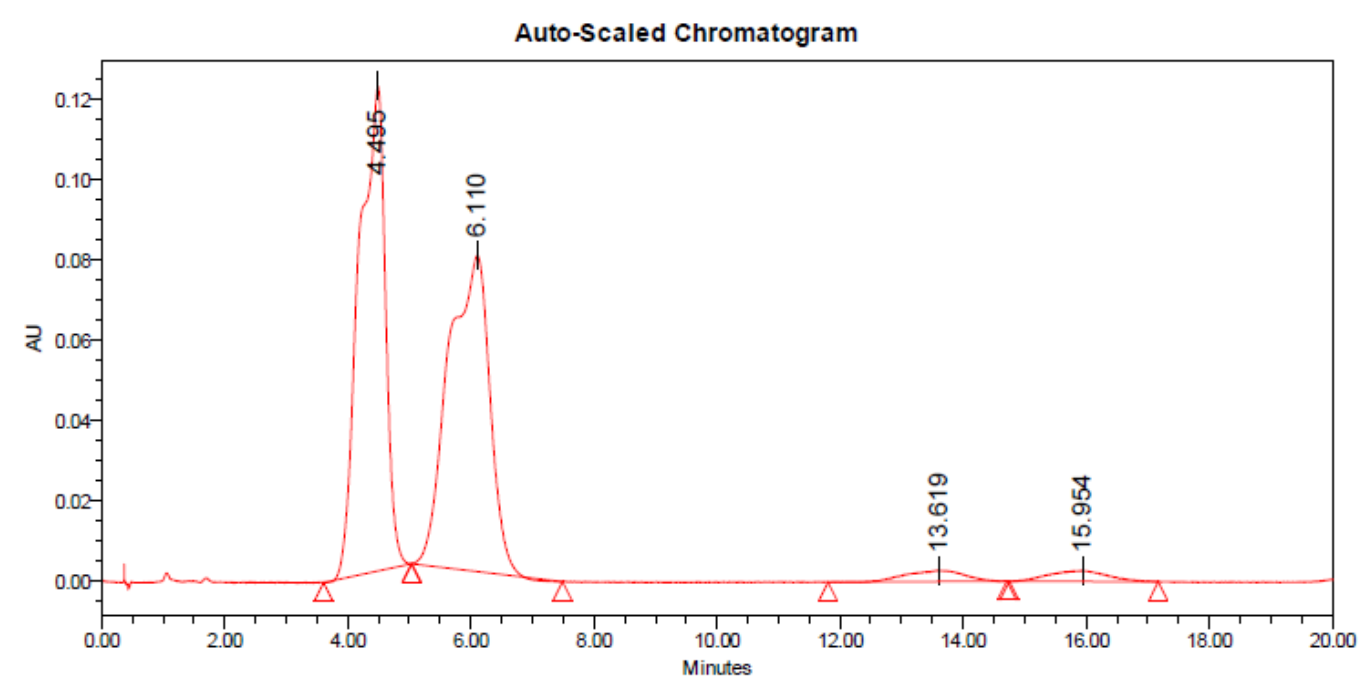

Processed Channel: PDA Ch1 254nm@1.2nm

-Compens.

\begin{tabular}{|l|l|r|r|r|r|}
\hline & Processed Channel & $\begin{array}{r}\text { Retention } \\
\text { Time }(\mathrm{min})\end{array}$ & Area & \% Area & Height \\
\hline 1 & PDA Ch1 254nm@1.2nm -Compens. & 4.495 & 3579355 & 47.02 & 120694 \\
\hline 2 & PDA Ch1 254nm@1.2nm -Compens. & 6.110 & 3676327 & 48.30 & 78772 \\
\hline 3 & PDA Ch1 254nm@1.2nm -Compens. & 13.619 & 180970 & 2.38 & 2807 \\
\hline 4 & PDA Ch1 254nm@1.2nm-Compens. & 15.954 & 175553 & 2.31 & 2776 \\
\hline
\end{tabular}

Auto-Scaled Chromatogram

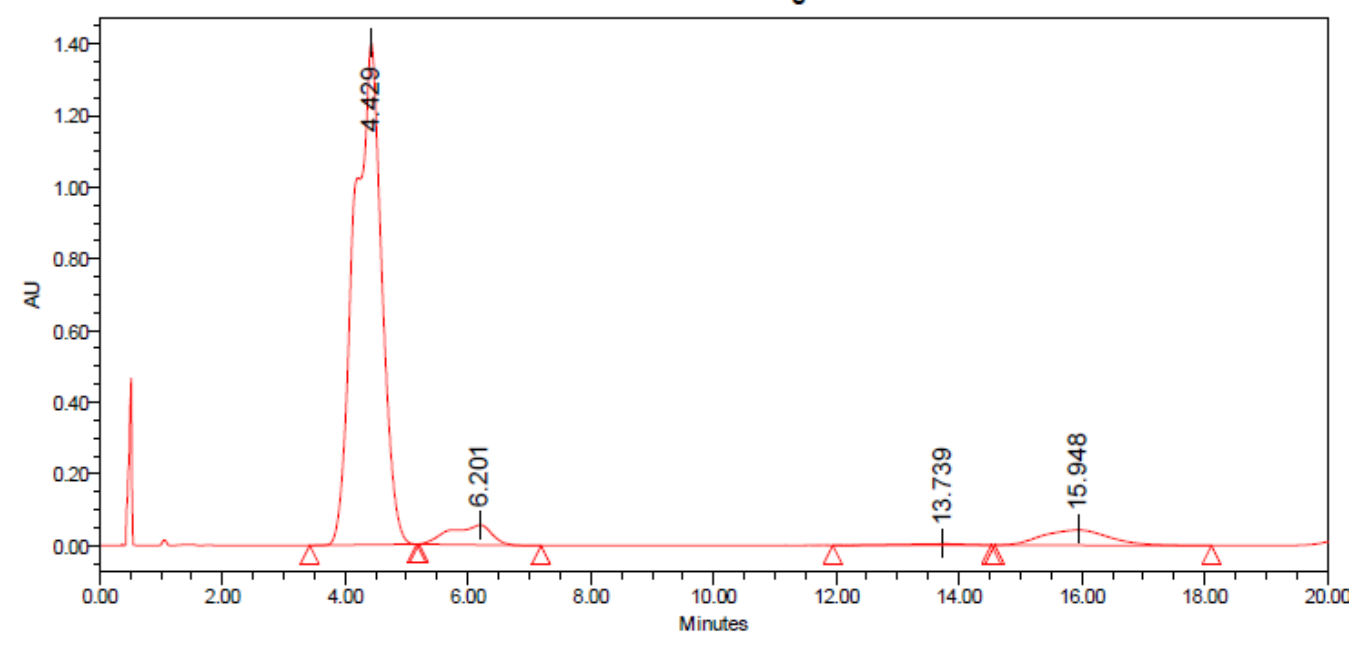

Processed Channel: PDA Ch1 254nm@1.2nm

-Compens.

\begin{tabular}{|l|c|r|r|r|r|}
\hline & Processed Channel & $\begin{array}{c}\text { Retention } \\
\text { Time (min) }\end{array}$ & Area & $\%$ Area & Height \\
\hline 1 & PDA Ch1 254nm@1.2nm -Compens. & 4.429 & 43295030 & 87.81 & 1398181 \\
\hline 2 & PDA Ch1 254nm@1.2nm -Compens. & 6.201 & 2612008 & 5.30 & 55403 \\
\hline 3 & PDA Ch1 254nm@1.2nm -Compens. & 13.739 & 213676 & 0.43 & 3285 \\
\hline 4 & PDA Ch1 254nm@1.2nm -Compens. & 15.948 & 3184060 & 6.46 & 43697 \\
\hline
\end{tabular}


4u - Chiral SFC (OJ3 column, flow rate $=2 \mathrm{~mL} / \mathrm{min}, \mathrm{T}=40{ }^{\circ} \mathrm{C}, 8 \%$ methanol $/ \mathrm{CO}_{2}, \mathrm{t}=10 \mathrm{~min}$ ): major diastereomer: major at $2.4 \mathrm{~min}$, minor at $5.5 \mathrm{~min}, 92 \%$ ee and minor diastereoisomer. major at $4.5 \mathrm{~min}$, minor at $3.2 \mathrm{~min}, 55 \%$ ee.

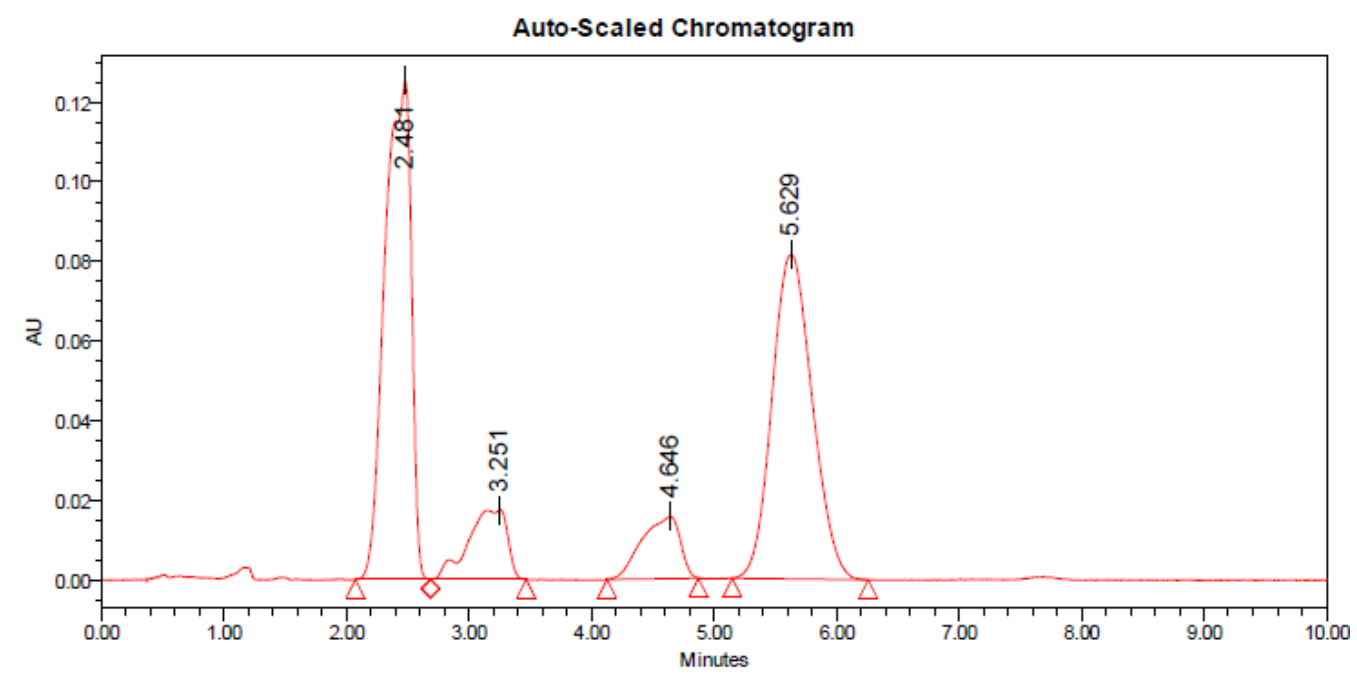

Processed Channel: PDA Ch1 254nm@1.2nm -Compens.

\begin{tabular}{|c|c|r|r|r|r|}
\hline & Processed Channel & $\begin{array}{c}\text { Retention } \\
\text { Time (min) }\end{array}$ & Area & \% Area & Height \\
\hline 1 & PDA Ch1 254nm@1.2nm -Compens. & 2.481 & 1872334 & 41.95 & 125227 \\
\hline 2 & PDA Ch1 254nm@1.2nm -Compens. & 3.251 & 394096 & 8.83 & 17534 \\
\hline 3 & PDA Ch1 254nm@1.2nm -Compens. & 4.646 & 336812 & 7.55 & 15576 \\
\hline 4 & PDA Ch1 254nm@1.2nm -Compens. & 5.629 & 1859686 & 41.67 & 81451 \\
\hline
\end{tabular}

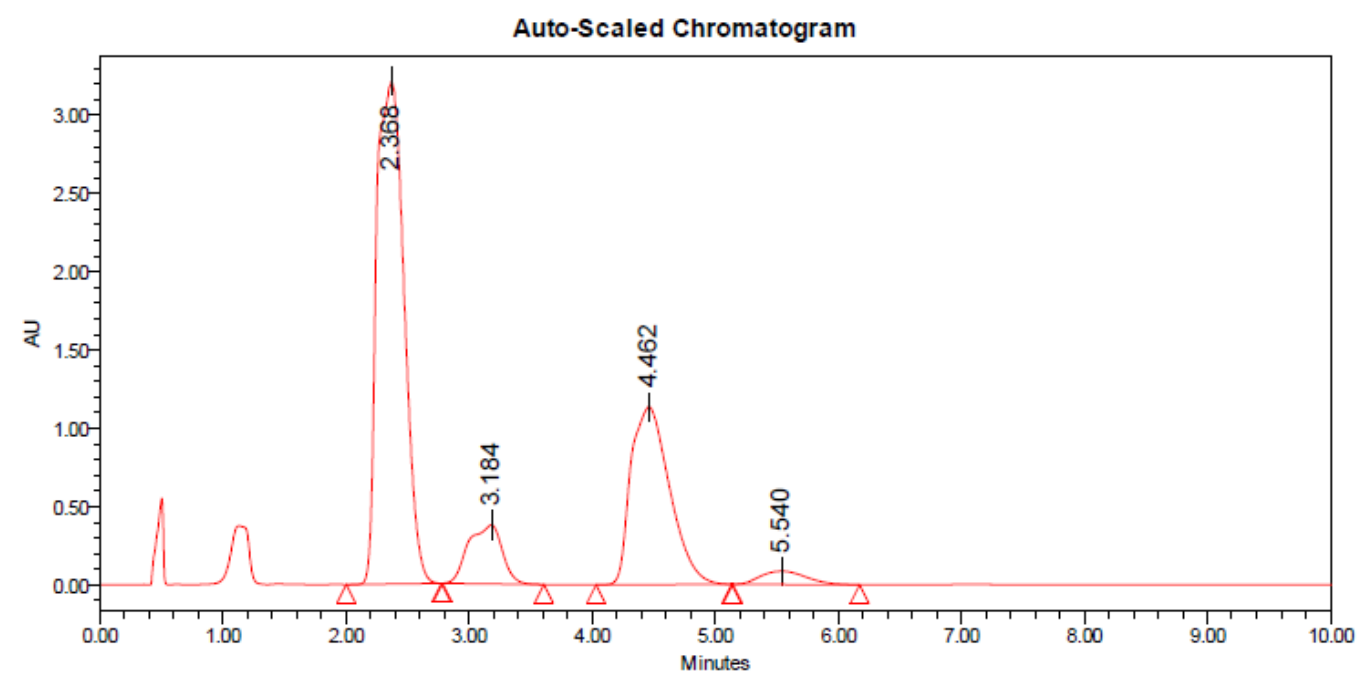

Processed Channel: PDA Ch1 254nm@1.2nm -Compens.

\begin{tabular}{|l|l|r|r|r|r|}
\hline & Processed Channel & $\begin{array}{r}\text { Retention } \\
\text { Time ( } \min )\end{array}$ & Area & $\%$ Area & Height \\
\hline 1 & PDA Ch1 254nm@1.2nm -Compens. & 2.368 & 49386543 & 59.11 & 3209622 \\
\hline 2 & PDA Ch1 254nm@1.2nm -Compens. & 3.184 & 7151685 & 8.56 & 376876 \\
\hline 3 & PDA Ch1 254nm@1.2nm -Compens. & 4.462 & 24836111 & 29.73 & 1137620 \\
\hline 4 & PDA Ch1 254nm@1.2nm -Compens. & 5.540 & 2174536 & 2.60 & 85222 \\
\hline
\end{tabular}


$4 \mathbf{v}$ - Chiral SFC (OJ3 column, flow rate $=2 \mathrm{~mL} / \mathrm{min}, \mathrm{T}=40{ }^{\circ} \mathrm{C}, 20 \%$ methanol $/ \mathrm{CO}_{2}, \mathrm{t}=10 \mathrm{~min}$ ): major diastereomer: major at $1.8 \mathrm{~min}$, minor at $1.7 \mathrm{~min}, 82 \%$ ee and minor diastereoisomer. major at $2.9 \mathrm{~min}$, minor at $2.4 \mathrm{~min}, 66 \%$ eee.

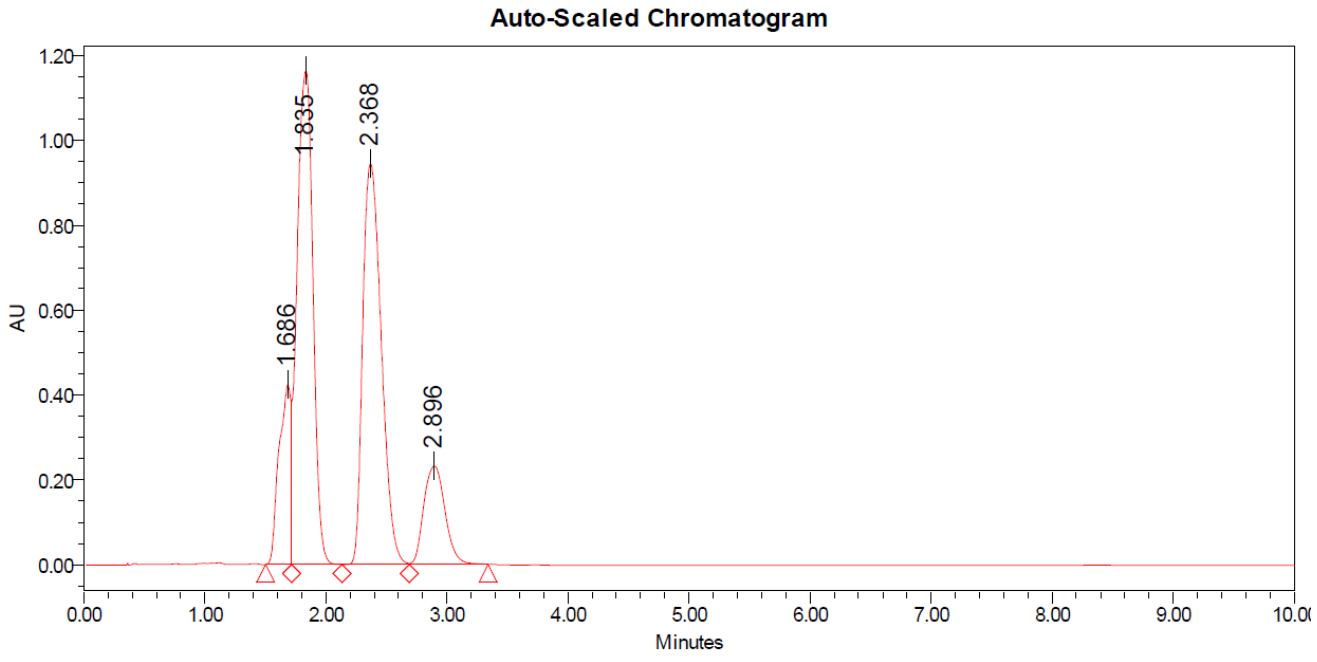

Processed Channel: PDA Ch1 254nm@1.2nm -Compens.

\begin{tabular}{|l|l|r|r|r|r|}
\hline & Processed Channel & $\begin{array}{r}\text { Retention } \\
\text { Time }(\min )\end{array}$ & Area & $\%$ Area & Height \\
\hline 1 & PDA Ch1 254nm@1.2nm-Compens. & 1.686 & 2872408 & 10.88 & 423307 \\
\hline 2 & PDA Ch1 254nm@1.2nm-Compens. & 1.835 & 10662555 & 40.38 & 116302 \\
\hline 3 & PDA Ch1 254nm@1.2nm-Compens. & 2.368 & 10042138 & 38.03 & 943922 \\
\hline 4 & PDA Ch1 254nm@1.2nm-Compens. & 2.896 & 2826483 & 10.70 & 231380 \\
\hline
\end{tabular}

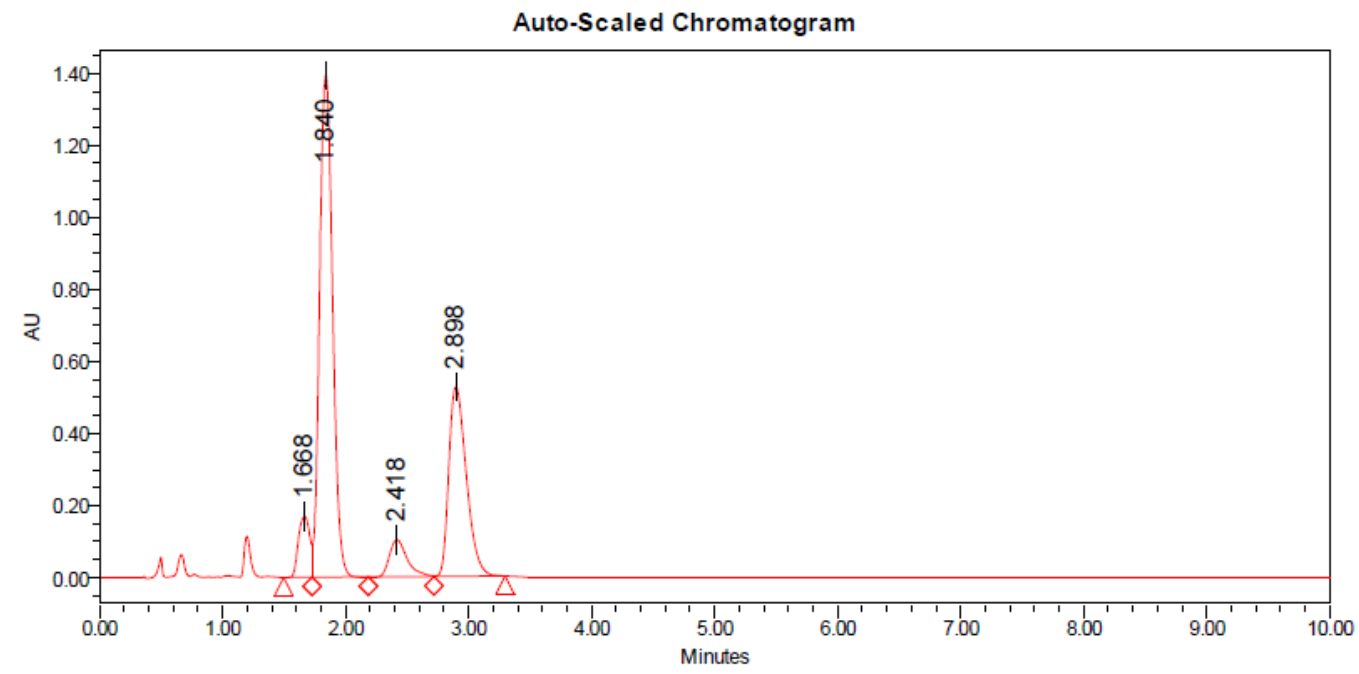

Processed Channel: PDA Ch1 254nm@1.2nm -Compens.

\begin{tabular}{|l|l|r|r|r|r|}
\hline & Processed Channel & $\begin{array}{c}\text { Retention } \\
\text { Time ( } \min )\end{array}$ & Area & $\%$ Area & Height \\
\hline 1 & PDA Ch1 254nm@1.2nm-Compens. & 1.668 & 1063224 & 6.07 & 168376 \\
\hline 2 & PDA Ch1 254nm@1.2nm -Compens. & 1.840 & 10230547 & 58.39 & 1392815 \\
\hline 3 & PDA Ch1 254nm@1.2nm-Compens. & 2.418 & 1022668 & 5.84 & 102154 \\
\hline 4 & PDA Ch1 254nm@1.2nm-Compens. & 2.898 & 5205817 & 29.71 & 525397 \\
\hline
\end{tabular}


9 - Chiral HPLC (IA column, flow rate $=1 \mathrm{~mL} / \mathrm{min}, \mathrm{T}=25^{\circ} \mathrm{C}, 20 \%$ IPA $/ n$-hexane, $\mathrm{t}=60 \mathrm{~min}$ ): major diastereomer: major at 14.9 min, minor at $23.2 \mathrm{~min}, 97 \%$ ee and minor diastereoisomer: major at $17.6 \mathrm{~min}$, minor at $31.8 \mathrm{~min}, 30 \%$ ee.

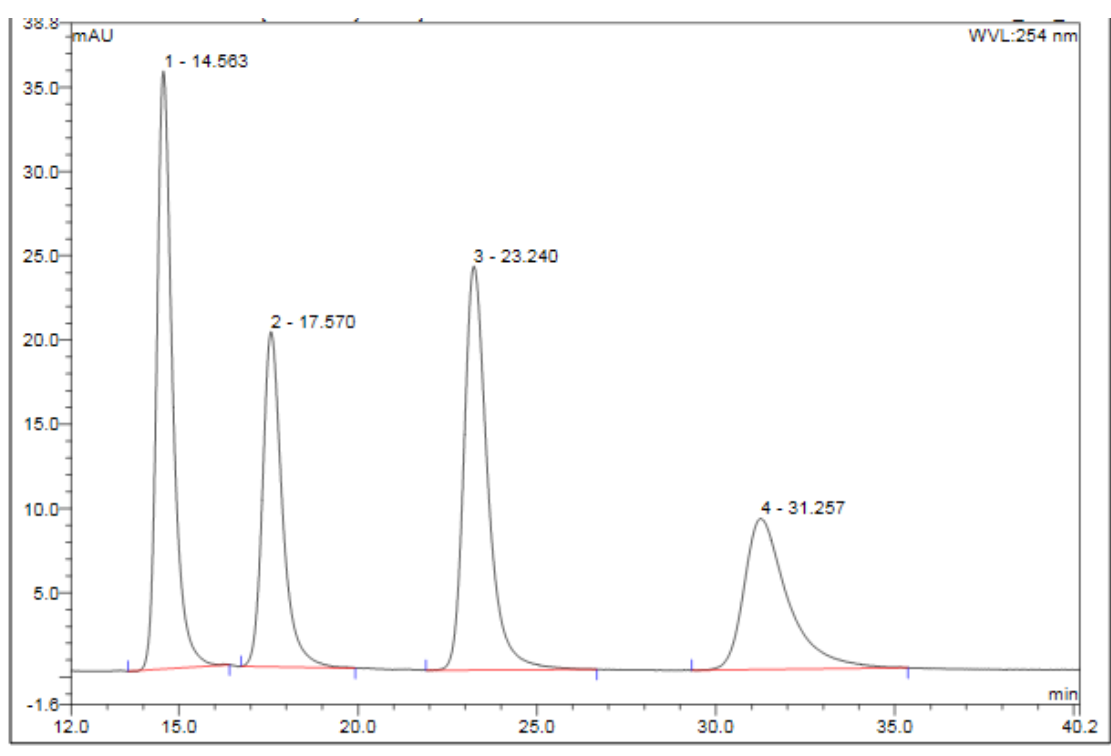

\begin{tabular}{|r|ccrrrrr|}
\hline No. & $\begin{array}{c}\text { Ret.Time } \\
\text { min }\end{array}$ & Peak Name & $\begin{array}{c}\text { Height } \\
\text { mAU }\end{array}$ & $\begin{array}{c}\text { Area } \\
\text { mAU*min }\end{array}$ & $\begin{array}{c}\text { Rel.Area } \\
\%\end{array}$ & Amount & Type \\
\hline 1 & 14.56 & n.a. & 35.480 & 18.173 & 29.36 & n.a. & BMB $^{\star}$ \\
2 & 17.57 & n.a. & 19.880 & 12.692 & 20.51 & n.a. & BMB $^{\star}$ \\
3 & 23.24 & n.a. & 23.963 & 18.337 & 29.63 & n.a. & BMB $^{\star}$ \\
4 & 31.26 & n.a. & 8.954 & 12.694 & 20.51 & n.a. & BMB $^{\star}$ \\
\hline Total: & & & 88.276 & 61.896 & 100.00 & 0.000 & \\
\hline
\end{tabular}

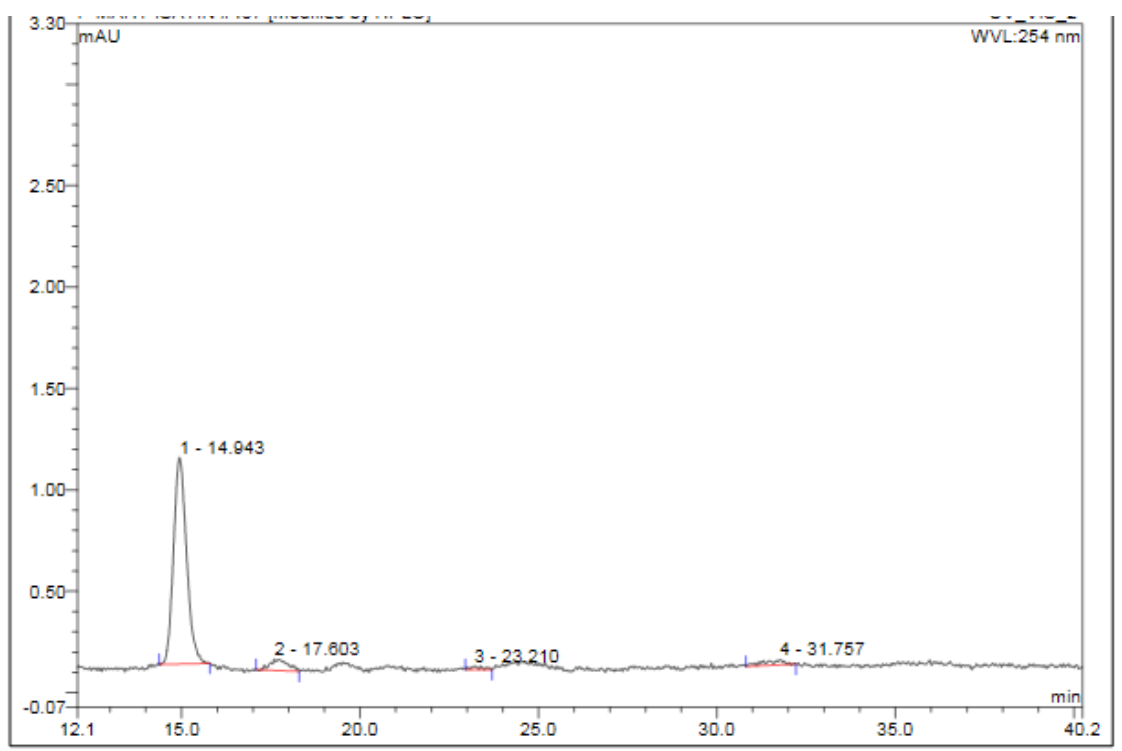

\begin{tabular}{|r|ccrrrrr|}
\hline No. & $\begin{array}{c}\text { Ret.Time } \\
\text { min }\end{array}$ & Peak Name & $\begin{array}{c}\text { Height } \\
\text { mAU }\end{array}$ & $\begin{array}{c}\text { Area } \\
\text { mAU*min }\end{array}$ & $\begin{array}{r}\text { Rel.Area } \\
\%\end{array}$ & Amount & Type \\
\hline 1 & 14.94 & n.a. & 1.017 & 0.430 & 88.73 & n.a. & BMB $^{\star}$ \\
2 & 17.60 & n.a. & 0.055 & 0.031 & 6.48 & n.a. & BMB $^{\star}$ \\
3 & 23.21 & n.a. & 0.018 & 0.006 & 1.29 & n.a. & BMB $^{\star}$ \\
4 & 31.76 & n.a. & 0.025 & 0.017 & 3.50 & n.a. & BMB $^{\star}$ \\
\hline Total: & & & 1.116 & 0.485 & 100.00 & 0.000 & \\
\hline
\end{tabular}




\section{References}

(1) (a) McCooey, S. H.; Connon, S. J. Urea- and Thiourea-Substituted Cinchona Alkaloid Derivatives as Highly Efficient Bifunctional Organocatalysts for the Asymmetric Addition of Malonate to Nitroalkenes: Inversion of Configuration at C9 Dramatically Improves Catalyst Performance. Angew. Chem. Int. Ed. 2005, 44, 6367-6270; (b) Corti, V.; Camarero Gonzalez, P.; Febvay, J.; Caruana, L.; Mazzanti, A.; Fochi M.; Bernardi L. Organocatalytic Asymmetric Sulfa-Michael Addition of 2-Aminothiophenols to Chalcones: First Enantioselective Access to 2,3,4,5-Tetrahydro-1,5-benzothiazepines. Eur. J. Org. Chem. 2017 49-52.

(2) Saadi, J.; Wennemers, H. Enantioselective aldol reactions with masked fluoroacetates. Nat. Chem. 2016, 8, 276-280.

(3) the reaction in acetophenone was performed at room temperature

(4) Luo, J.; Gao, S.; Ma, Y.; Ge, G. Palladium-Catalyzed oxidation of indoles to isatins by tert-butyl hydroperoxide. Synlett 2018, 29, 969-973.

(5) Liao, Y. Y.; Gao, Y. C.; Zheng, W.; Tang, R. Y. Oxidative Radical Cyclization of N-methyl-Narylpropiolamide to Isatins via Cleavage of the Carbon-carbon Triple Bond. Adv. Synth. Catal. 2018, 360, 3391-3400.

(6) Laina-Martín, V.; Humbrías-Martín, J.; Fernández-Salas, J. A.; Alemán, J. Asymmetric vinylogous Mukaiyama aldol reaction of isatins under bifunctional organocatalysis: enantioselective synthesis of substituted 3-hydroxy-2-oxindoles. Chem. Comm. 2018, 54, 2781-2784.

(7) Ji, H.; Zhu, Y.; Shao, Y. ; Liu, J.; Yuan, Y.; Jia, X. Active sp3 C-H Bond Oxidation Initiated sp3-sp2 Consecutive $\mathrm{C}-\mathrm{H}$ Functionalization of N-Arylglycine Amides: Construction of Isatins. J. Org. Chem. 2017, 82, 9859-9865.

(8) Dolomanov, O.V., Bourhis, L.J., Gildea, R.J, Howard, J.A.K. \& Puschmann, H. OLEX2: a complete structure solution, refinement and analysis program. J. Appl. Cryst. 2009, 42, 339-341.

(9) Sheldrick, G.M. SHELXT - Integrated space-group and crystal-structure determination Acta Cryst. 2015, A71, 3-8.

(10) Sheldrick, G.M. Crystal structure refinement with SHELXL. Acta Cryst. 2015, C71, 3-8. 